\title{
EFFECT OF THE KAIKŌURA EARTHQUAKE ON VELOCITY CHANGES IN AND AROUND THE RUPTURED REGION: A NOISE CROSS-CORRELATION APPROACH
}

BY

MEGAN ANN KORTINK

\begin{abstract}
A thesis
submitted to the Victoria University of Wellington in partial fulfillment of the requirements for the degree of Masters of Science in Geophysics
\end{abstract}

Victoria University of Wellington 2020 



\section{Abstract}

Seismic velocity changes before and after large magnitude earthquakes carry information about damage present within the faults in the surrounding region. In this thesis, temporal velocity changes are measured before and after the 2016 Kaikōura earthquake using ambient noise interferometry between $2012-2018$. This period contains the $M_{w} 7.82016$ Kaikōura earthquake as well as the 2013 Cook Strait earthquake sequence and a few deep large magnitude earthquakes in 2015 - 2016 . Three primary objectives are identified: (1) investigate seismic velocity changes in the Kaikōura region and their connection to the 2016 Kaikōura earthquake to try and determine if there was a change before/after the earthquake, (2) determine how this change varied across the region, and (3) consider if ambient noise can lead to improved detection and understanding of geological hazard.

The primary approach used to measure velocity changes in the Kaikōura region involved cross correlating noise recorded by seismic stations across the region. Velocity changes are sought by averaging the best result from multiple onshore station pairs. A secondary approach was also used, in which specific station pairs were averaged to determine if there were more localised velocity changes over more specific regions. This was to determine if the velocity changes observed following the 2016 Kaikōura earthquake occurred over the entire ruptured region.

Following the 2016 Kaikōura earthquake a velocity decrease of $0.24 \pm 0.02 \%$ was observed on the average of the vertical-vertical components for eight stations. The remaining eight cross-component pairs showed a smaller seismic decrease with an average value of $0.22 \pm 0.05 \%$. After the decrease following the Kaikōura earthquake, 
there is a steady velocity increase of $0.13 \pm 0.02 \%$ over a one-and-a-half-year period. This indicates that prior to the earthquake, seismic velocity was at a steady state until it was perturbed by the Kaikōura earthquake, and seismic velocities rapidly decreased over all stations. Across the region, stations with a longer interstation distance and further away from ruptured faults had a smaller decrease in velocity than station pairs with a smaller interstation distance that were closer to ruptured faults. We interpret the velocity decrease following the Kaikōura earthquake as a result of cracks opening during the earthquake. The velocity increase following the earthquake is indicative of the cracks slowly healing.

The Cook Strait earthquake sequence that occurred in 2013 did not cause any velocity changes at the stations used in this thesis. This has been interpreted to be because the changes were too small compared to the background noise or the stations were not recording during the time of the earthquake sequence. Two other decreases were also observed in the region following two deep earthquakes in April $2015\left(M_{w} 6.2\right.$, depth $=52 \mathrm{~km})$ and February $2016\left(M_{w} 5.7\right.$, depth $\left.=48 \mathrm{~km}\right)$. Both of these events resulted in a small seismic decrease of $0.1 \pm 0.02 \%$. Although these earthquakes were close to seismic stations when they occurred, they were much deeper and had a smaller magnitude than the Kaikōura earthquake so did not cause a large velocity decrease. By understanding what causes velocity changes it is possible to have an improved understanding of the geological hazard in the region. 


\section{Acknowledgements}

Firstly, I would like to thank my supervisor Martha Savage for her guidance and support over the past year. Thank you for the opportunity to be part of this research project. Thank you to the Evison family and to EQC for providing me with financial support offered through the Frank Evison Research Scholarship and for supplementing my thesis grant. This support allowed me to complete my thesis without undue financial stress. A big thank you also to Kyoto University for providing the dataset that I worked on.

Thank you to my fellow Geophysics students at Victoria University who helped at some stage towards my thesis development, whether it was a helpful suggestion or answering a troubling question. I would like to thank Katie Woods for all the tea breaks and encouragement, and Alec Yates, Weiwei Wang, Katrina Jacobs, and Kenny Graham for helping me with coding or ambient noise related queries. I would also like to further thank Alec for letting me use his figures and for help in proofreading. I would also like to thank Aleksandr Beliaev for all of my numerous IT enquires. Also my thanks goes out to everyone in the SGEES office for helping me with all of my masters related questions.

To my parents and siblings, thank you for your unconditional love, support and encouragement during all of my university studies, especially my final year. Thank you for always pushing me to be my best!

Finally, I would also like to thank my partner Daniel Madley. Your support and encouragement, especially in the final weeks has kept me going. This journey would not have been possible without you or my family. 


\section{Contents}

Abstract $\quad$ i

Acknowledgements

List of Figures vii

List of Tables $\quad$ ix

1 Introduction $\quad 1$

1.1 Motivation . . . . . . . . . . . . . . . . . 1

1.2 Primary Objectives . . . . . . . . . . . . . . . . . . . 2

1.3 Kaikoura Region . . . . . . . . . . . . . . . . . . . . . . 3

1.3.1 Geological Setting . . . . . . . . . . . . . . . . 4

1.3.2 Recent Earthquakes . . . . . . . . . . . . . . . . 5

1.4 Study Design . . . . . . . . . . . . . . . . . . 8

1.5 Thesis Structure . . . . . . . . . . . . . . . . . . . . . 9

1.5.1 Glossary of Acronyms and Terms . . . . . . . . . . . 10

2 Background Theory 13

2.1 Ambient Noise Theory . . . . . . . . . . . . . . . . . . . . . . . 13

2.1.1 Ambient Seismic Noise Properties . . . . . . . . . . . . . . . . 14

2.1.2 Cross-Correlation Technique and Green's Function Recovery . 18

2.1.3 Seismic Velocity Variations . . . . . . . . . . . . . . . . . 23

2.2 Earthquakes and Ambient Noise . . . . . . . . . . . . . . . . . 27

2.2.1 Physical Process . . . . . . . . . . . . . . . . 27

2.2 .2 Previous Studies . . . . . . . . . . . . . . . . . . . . 29

$\begin{array}{lll}3 & \text { Data } & 35\end{array}$

$\begin{array}{llr}4 & \text { Methods } & 39\end{array}$

4.1 Processing Sequence . . . . . . . . . . . . . . . . . . . . . . . 39

4.1 Pre-processing . . . . . . . . . . . . . . . . . . . . . . . . . . . . 40

4.1.2 Cross-Correlation computation . . . . . . . . . . . . 44

4.1.3 Moving and Reference Stacks . . . . . . . . . . . . . . . . . 45

4.1 .4 Velocity Variations . . . . . . . . . . . . . . . . . 46

4.2 Parameter Justification . . . . . . . . . . . . . . . . . . . 47

4.2.1 Initial Testing - seven stations, four months of data, ZZ component . . . . . . . . . . . . . . . 48

4.2.2 Filter Frequencies and Coherent Noise . . . . . . . . . . . 49

4.2 .3 Parameter Processing with SNR . . . . . . . . . . . . 51 
4.2.4 Initial Moving-Window-Cross-Spectral Parameters . . . . . . . 53

4.2.5 Testing with two years of data over seven stations - prior to adding all stations . . . . . . . . . . . . 55

4.2.6 Testing with all of the stations for two years . . . . . . . . 55

4.2.7 Cross-Component tests for two years . . . . . . . . . . . . 68

4.2.8 Full Dataset — seven years, 12 stations . . . . . . . . . . 73

5 Results $\quad \mathbf{7 5}$

5.1 Stability of Velocity Changes . . . . . . . . . . . . . . . . . . 75

5.2 Velocity Changes for two years . . . . . . . . . . . . . . . 79

5.2 .1 Stations Used . . . . . . . . . . . . . . . . 79

5.2.2 Velocity Changes for the ZZ component . . . . . . . . . . 80

5.2.3 Velocity Changes for all cross-components . . . . . . . . . . . 84

5.3 Velocity Changes for seven years . . . . . . . . . . . . . . 87

5.3 .1 Stations Used . . . . . . . . . . . . . . . . 88

5.3.2 Velocity Changes for the ZZ component . . . . . . . . . . 89

5.3.3 Velocity Changes for all cross-components . . . . . . . . . . 93

6 Discussion $\quad 99$

6.1 Sampling Depth and Location . . . . . . . . . . . . . . . . . 100

6.1.1 Depth Sensitivity Kernels . . . . . . . . . . . . . . . 100

6.1 .2 Velocity Changes across the South Island . . . . . . . . . . . 103

6.2 Velocity Changes . . . . . . . . . . . . . . . . . . . 108

6.2.1 Cook Strait earthquake sequence 2013 . . . . . . . . . . 111

6.2.2 Earthquake in April 2015 and February 2016 . . . . . . . . . . 114

6.2.3 The 2016 Kaikōura earthquake . . . . . . . . . . . . . . . 116

6.2.4 Mechanisms for velocity changes . . . . . . . . . . . . . 117

6.3 Comparison to Previous Studies . . . . . . . . . . . . . . . . . . 120

6.4 Geological Hazard Monitoring . . . . . . . . . . . . . . . . . . . . . . 124

$\begin{array}{llr}7 & \text { Conclusions } & 127\end{array}$

7.1 Summary of Findings . . . . . . . . . . . . . . . . . . . . . . 128

7.1.1 Kaikōura earthquake . . . . . . . . . . . . . . . . . . . . 128

7.1.2 Regional Seismic Changes . . . . . . . . . . . . . . . . . . 129

7.1.3 Geological Hazard . . . . . . . . . . . . . . . . . . . . . . . 129

7.2 Recommendations for Further Work . . . . . . . . . . . . . . . . 130

$\begin{array}{ll}\text { A Seismic Station Information } & 149\end{array}$

$\begin{array}{ll}\text { B Final Parameter Choices } & 150\end{array}$ 


\section{List of Figures}

1.1 New Zealand Geological Map. . . . . . . . . . . . . . . . . . . . . . . 3

1.2 Known and ruptured faults in the Kaikōura region. . . . . . . . . . . 4

1.3 Historical Earthquake Map from 1840 to 2018. . . . . . . . . . . . . . 7

1.4 Seismic stations used in this thesis. . . . . . . . . . . . . . 8

2.1 Primary and secondary microseisms in a continuous seismic recording. 16

2.2 Illustration of the cross-correlation of seismic energy travelling between two receivers. . . . . . . . . . . . . . . . . . . 21

2.3 Positive and Negative time lags for a cross-correlation function. . . . 22

2.4 Illustration of how velocity changes are recovered from ambient noise CCF. . . . . . . . . . . . . . . . 25

3.1 Location of the DPRI stations. . . . . . . . . . . . 36

3.2 DPRI station availability. . . . . . . . . . . . . . . . . . 38

4.1 Different methods of temporal normalisation. . . . . . . . . . . . . . . 43

4.2 Moving-window cross-spectral analysis example, with a slope (red line) and its uncertainty (dashed red lines) fitted to delay values. . . . . . 46

4.3 Filter Sequencing. . . . . . . . . . . . . . . . . . . . . 49

4.4 Spectrogram showing energy frequencies. . . . . . . . . . . . . 50

4.5 Distance plot showing arrivals at an increasing interstation distance. . 51

4.6 Initial pre-process bandpass filters. . . . . . . . . . . . . . . 53

4.7 SNR parameter tests analysing filters, normalisation, different phase weightings and cross-correlation time slice values. . . . . . . . . . . 54

4.8 Correlation coefficient plot for CCB-CVR. . . . . . . . . . . . . 57

4.9 Correlation coefficient plot for SJQ-WJM with a relatively low filter. 58

4.10 Correlation coefficient plot for SJQ-WJM with a relatively high filter. 58

4.11 SNR Filters Test showing variability in frequency range values. . . . . 60

4.12 Coherency Filters Test showing variability in frequency range, MWCS window length and MWCS step. . . . . . . . . . . . . . . . 60

4.13 Comparing SNR against coherency for the same filters. . . . . . . . . 61

4.14 SNR plot showing cross-correlation time slice values. . . . . . . . . . 62

4.15 Cross-correlation stability and quality for station pair IKR-MLF. . . 63

4.16 Cross-correlation stability and quality for station pair SJQ-WJM. . . 64

4.17 Distance plot of dynamic and static shift. . . . . . . . . . . . . . 66

4.18 DVV plot showing forced and unforced linear regression for a 10-day and 30-day moving window. . . . . . . . . . . . 66 66

4.19 DVV plot for the East-East component. . . . . . . . . . . . . . . 69

4.20 DVV plot for the North-North component. . . . . . . . . . . . . . . . 69

4.21 DVV plot for the East-North component. . . . . . . . . . . . . . . 70 
4.22 DVV plot for the North-East component. . . . . . . . . . . . . . . . . 70

4.23 DVV plot for the East-Vertical component. . . . . . . . . . . . . . . 71

4.24 DVV plot for the Vertical-East component. . . . . . . . . . . . . . . 71

4.25 DVV plot for the North-Vertical component. . . . . . . . . . . . . . . 72

4.26 DVV plot for the Vertical-North component. . . . . . . . . . . . . . . 72

4.27 Velocity changes for the vertical-vertical component with a two-and-ahalf-year reference period for a 30-day moving window for a seven-year period. . . . . . . . . . . . . . . . 74

5.1 Reference cross-correlation functions against interstation distance for the seven-year dataset. . . . . . . . . . . . . . . 77

5.2 SNR plot for all nine cross-components. . . . . . . . . . . . . . . 78

5.3 Velocity changes using a two-year dataset for different moving windows. 81

5.4 Velocity changes using a two-year dataset for the ZZ component using a two-and-a-half-year reference period. . . . . . . . . . . . . 82

5.5 Velocity changes using a six-month and a two-year reference period for different moving windows. . . . . . . . . . . . . . . . . 83

5.6 Velocity changes using a six-month and a two-year reference period for a 30-day moving window. . . . . . . . . . . . . . . . . . 84

5.7 Velocity changes for nine cross-components using a six-month reference period. . . . . . . . . . . . . . . . . 85

5.8 Velocity variations for different cross-component pairs using a 30-day moving window and a six-month reference period. . . . . . . . . .88 86

5.9 Velocity changes for the ZZ component using a 30-day moving window for a two-and-a-half-year reference period. . . . . . . . . . . . . . 90

5.10 Velocity changes for the ZZ component using different moving windows. 91

5.11 Velocity changes for the ZZ component for different stations and reference periods. . . . . . . . . . . . . . . . . . . 92

5.12 Velocity variations for all nine cross-components using a seven-year reference period. . . . . . . . . . . . . . . . 93

5.13 Velocity variation figures for all cross-components for a 30-day moving window with a seven-year reference period. . . . . . . . . . . . 95

6.1 Velocity variation for four different frequency ranges. . . . . . . . . . 101

6.2 Depth sensitivity kernels. . . . . . . . . . . . . . . . . . . 103

6.3 Station map divided into the four analysed regions. . . . . . . . . . . 104

6.4 Velocity variation for station pairs within each quadrant. . . . . . . . 105

6.5 Velocity variation for station pairs across each quadrant. . . . . . . . 107

6.6 Map with earthquake locations greater than Magnitude 5.5 from 2012-01-01 to 2018-02-28. . . . . . . . . . . . . . . . . . . . . 110

6.7 Velocity variation plot of median and mean velocity changes for 30-day, 60-day and 90-day moving windows. . . . . . . . . . . . . 111

6.8 Velocity variation plot of median velocity changes for 30-day, 60-day and 90-day moving windows. . . . . . . . . . . . . . . . 112

6.9 Velocity variation of station pairs close to the 2013 Cook Strait earthquake sequence. . . . . . . . . . . . . . . . . . . 113

6.10 Velocity variation of station pairs close to the April 2015 and February 2016 earthquakes. . . . . . . . . . . . . . . . 115

6.11 Several possible mechanisms for velocity change. . . . . . . . . . . . 119 


\section{List of Tables}

1.1 Glossary of Acronyms . . . . . . . . . . . . . . . . . . . . . 11

1.2 Glossary of Terms . . . . . . . . . . . . . . . . . . . 12

2.1 Summary by Bonnefoy-Claudet et al. (2006) established from studies by Gutenberg (1958), Asten (1978) and Asten and Henstridge (1984). 15

4.1 Table of filters used to test the ZZ component for the two-year dataset. 59

4.2 Stations for different cross-components for the two-year dataset. . . . 68

4.3 Table of filters used to test all cross-component pairs for the two-year dataset. . . . . . . . . . . . . . . . . . . 68

5.1 Stations for the two-year dataset. . . . . . . . . . . . . . . 80

5.2 Stations for the seven-year dataset. . . . . . . . . . . . . . 88

6.1 Table describing the numbers annotating earthquakes in Figure 6.6. . 109

6.2 Relative velocity decreases by cross-correlation component for all stations averaged from the beginning of the decrease to the end of the decrease. . . . . . . . . . . . . . . . 116

6.3 Comparison of Previous Studies. . . . . . . . . . . . . . . . . . 122

A.1 Table of stations with unused data. . . . . . . . . . . . . . 149

B.1 Station pair pre-processing parameters. . . . . . . . . . . . . 151

B.2 Station pair dataset filter table. . . . . . . . . . . . . . . 151 


\section{Chapter 1}

\section{Introduction}

This chapter begins by covering the primary motivations and objectives of this thesis (Section 1.1 and 1.2). The Kaikōura region is introduced as well as recent earthquakes that have occurred here (Section 1.3). Finally, we conclude by describing the study design (Section 1.4) and the thesis structure (Section 1.5).

\subsection{Motivation}

The earthquake cycle is governed by the build-up and release of stress on faults. Understanding this process can be difficult because of subtle factors such as stresses closing and opening some fractures but not all; these open fractures then lead to fluid pathways which can lubricate faults, changing the stress field and how faults are damaged. To determine changes in a stress field, velocity changes will be used, as observed seismic velocity changes after earthquakes can be governed by the postseismic stress relaxation within deeper parts of the fault zone (Brenguier et al., 2008a). Measuring stress is a fundamental problem in earthquake science and so to help solve this problem we will be using changes in seismic velocity to measure the changes in how faults are damaged before and after an earthquake.

Changes in seismic velocity can be determined from seismic energy. Background seismic energy or seismic ambient noise, is generated from activity. Ambient Noise 
Theory is discussed further in Chapter 2. Four possible mechanisms for velocity changes associated with earthquakes are also discussed in Chapter 2. These mechanisms include: stress-induced changes, damage of the crust from the fault zone rupture, damage of the shallow crust from strong ground shaking, and fluid activity in the near surface and the crust.

Kaikōura is an excellent location to study ambient noise as there has recently been a large magnitude $\left(M_{w} 7.8\right)$ earthquake that ruptured over 21 faults (Stirling et al., 2017) in the surrounding region (Figure 1.2). Due to previous large magnitude events this region has experienced, many seismometers were already deployed prior to the Kaikōura earthquake, this resulted in a rich dataset. This dataset therefore will allow us insights into changes that occurred before, during, and after the Kaikōura earthquake.

\section{$1.2 \quad$ Primary Objectives}

The three key objectives of this study are delineated below. These will all be used to understand how stress affected the Kaikōura region and how this could lead to improved detection and understanding of New Zealand's geological hazard and assess the modelling of New Zealand's geohazard risk.

1. Investigate seismic velocity changes in the Kaikōura region and their connection to the 2016 Kaikōura earthquake. Attempt to determine if there was a change before/after the earthquake.

2. Determine how this change varied across the region.

3. Consider if ambient noise can lead to improved detection and understanding of geological hazard and if this could result in enhanced assessment and modelling of New Zealand's geohazard risk. 


\subsection{Kaikōura Region}

Kaikōura is part of the Marlborough Fault System (MFS) in the northeast of the South Island (Figure 1.1). The northeastern Marlborough region represents a transition from the oblique continental collision along the Alpine Fault in the south to subduction along the Hikurangi subduction zone in the north (Clark et al., 2017). The MFS is dominated by four oblique, dextral strike-slip faults (the Alpine, Awatere, Clarence, and Hope Faults (Rattenbury et al., 2006)) that accommodate $80 \%$ of the motion across the plates in the northern South Island (see Figure 1.1) (Van Dissen and Yeats, 1991; Wallace et al., 2012). These strike parallel to the Australian-Pacific plate motion, leading to predominantly right lateral strike-slip motion (Figure 1.2)

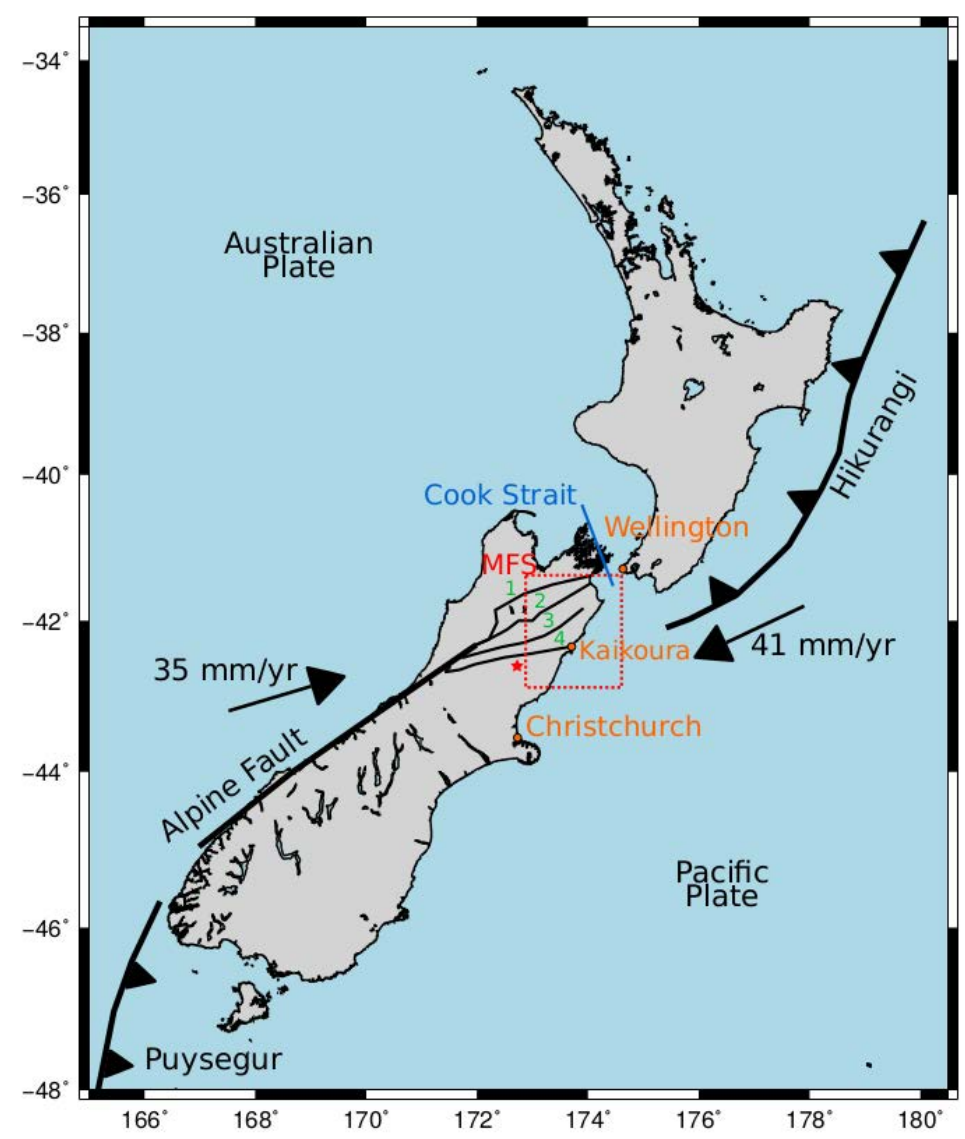

Figure 1.1: New Zealand Geological Map. The plate boundary with the Pacific and Australian plate shown by the black line with the convergence rates. The location of the Marlborough Fault System (MFS) is in the dashed red box. The location of the 2016 Kaikōura earthquake is indicated by the red star. The faults within the MFS are indicated by the green numbers, $1=$ Alpine Fault, 2=Awatere Fault, $3=$ Clarence Fault, 4=Hope Fault. 
(Wallace et al., 2012). The most recent large earthquake in the Kaikōura region was in 2016 with a magnitude of 7.8 (Figures 1.2 and 1.3). In this section, the geological setting of the Kaikōura region is discussed along with previous earthquakes that drive this study.

\subsubsection{Geological Setting}

The Kaikōura region straddles the boundary between the Australian and Pacific plates, which are converging at approximately $40 \mathrm{~mm}$ per year (Figure 1.1) (Rattenbury et al., 2006). This area is also in the transition region between the subduction of the Pacific plate under the Australian plate (Hikurangi subduction zone to the North), to continental collision in the South (Alpine Fault).

The basement geology of the Kaikoura region is dominated by quartzofieldspathic sedimentary rocks of the Rakaia and Pahau terranes (Bradshaw, 1989; Rattenbury et al., 2006). These amalgamated to form the Torlesse composite terrane.

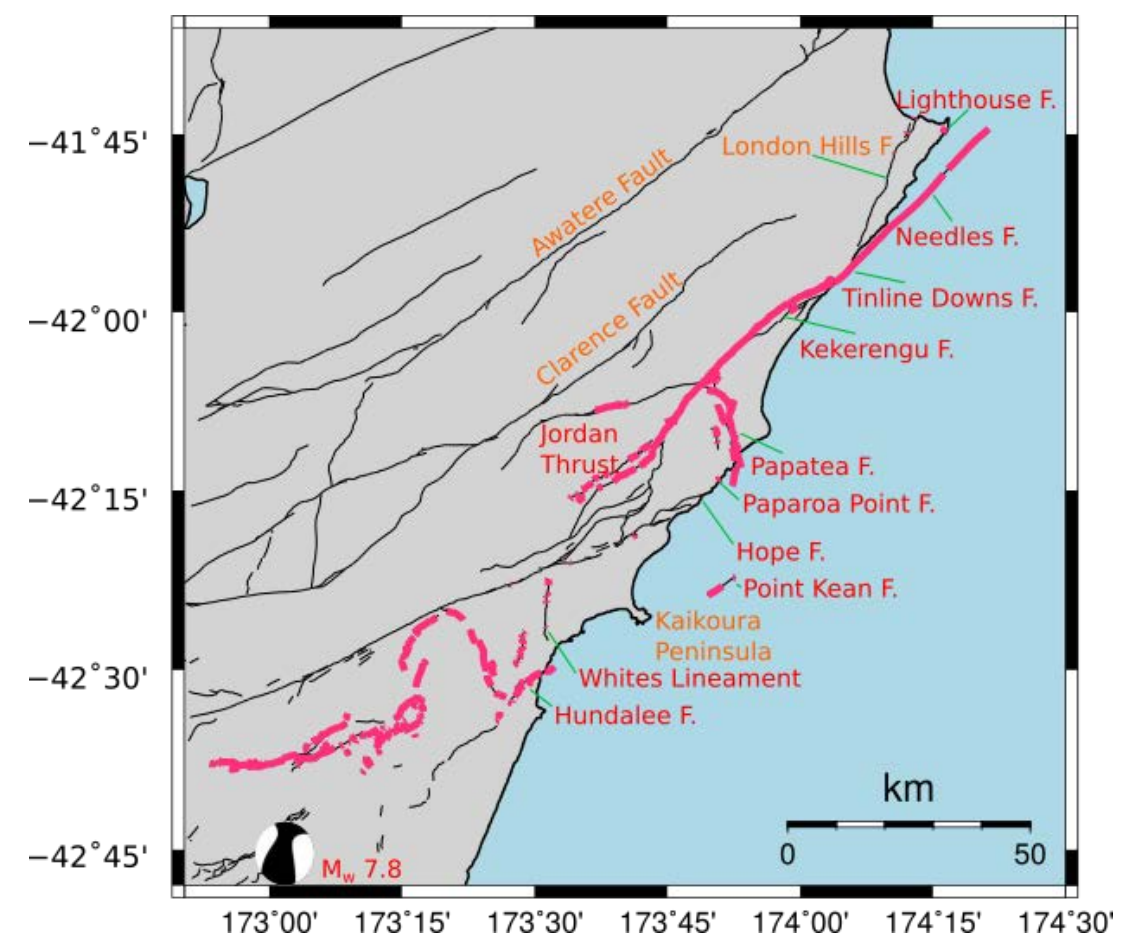

Figure 1.2: Known onshore faults in the Kaikōura region. The Kaikōura earthquake is shown by the focal mechanism from Hamling et al. (2017). Black lines are known active faults from Langridge et al. (2016) and the pink lines are the faults that ruptured during the 2016 Kaikōura mainshock (Litchfield et al., 2017). 


\subsubsection{Recent Earthquakes}

The most recent large earthquake (greater than $M_{w} 7$ ) in the Kaikōura region occurred shortly after midnight on the $14^{\text {th }}$ of November 2016 (Cesca et al., 2017; Clark et al., 2017; Hamling et al., 2017), with an epicentre $\sim 20 \mathrm{~km}$ south of the Hope Fault (Figure 1.2) (Hamling et al., 2017), near Waiau in north Canterbury (-42.693S, 173.022E from GeoNet). The earthquake had an oblique thrust mechanism (Clark et al., 2017) and the hypocentre was quite shallow at a depth of only $15 \mathrm{~km}$. The rupture propagated SW to NE in approximately two minutes and terminated offshore in the Cook Strait (Clark et al., 2017). From north Canterbury to the Cook Strait $(\sim 150 \mathrm{~km})$, there were surface ruptures on at least 21 separate faults (Stirling et al., 2017). There were also more surface ruptures offshore (Clark et al., 2017).

The Kaikōura region is situated within an area of high seismicity, as historical records indicate (Figure 1.3) (Rattenbury et al., 2006). Written records have been kept in New Zealand since 1840. During this time eleven shallow magnitude 6.0 or greater earthquakes have originated within the Kaikōura region. Four of these earthquakes, the 1848 Marlborough, the 1888 North Canterbury (Rattenbury et al., 2006), the 2010 Darfield earthquake (Gledhill et al., 2011) and the 2016 Kaikōura earthquake (Hamling et al., 2017) had magnitudes over 7.0. The 1848 Marlborough M 7.5 (Grapes et al., 1998) earthquake ruptured $\sim 100 \mathrm{~km}$ to the northeast of the 2016 Kaikōura earthquake (Duputel and Rivera, 2017) and ruptured $105 \mathrm{~km}$ (from the coast to Barefell Pass) of the Awatere Fault near Blenheim, with an average dextral displacement of $6 \mathrm{~m}$ (Grapes et al., 1998; Rattenbury et al., 2006). In 1888, Kaikōura was shaken by a magnitude $M$ 7-7.3 earthquake, $100 \mathrm{~km}$ north-north-west of Christchurch (Cowan, 1991), rupturing the central section of the Hope Fault (Stirling et al., 2008), a $\pm 30 \mathrm{~km}$ section west of Hanmer Springs (Rattenbury et al., 2006). As more earthquakes occur, more faults are discovered in the Marlborough region. After the most recent 2016 earthquake it is now thought that there are at least 21 separate onshore and offshore faults (Figure 1.2).

The Kaikōura region has also experienced many large shallow earthquakes which 
commonly result in more active faults on the surface. This results in more ground deformation than would usually occur with deeper earthquakes. Figure 1.3 also shows that there is a large cluster of historical earthquakes since 1840 that are shallower than $40 \mathrm{~km}$ within the MFS. In New Zealand, an active fault is by definition "a fault that has shown evidence of surface rupture or ground deformation within the last 125,000 years" (Langridge et al., 2016). This time period was selected as late Quaternary deposits during this period were generally widespread across New Zealand (Langridge et al., 2016) and thus can be used as a good marker for fault movements, particularly on alluvial terraces and fans (Rattenbury et al., 2006). 


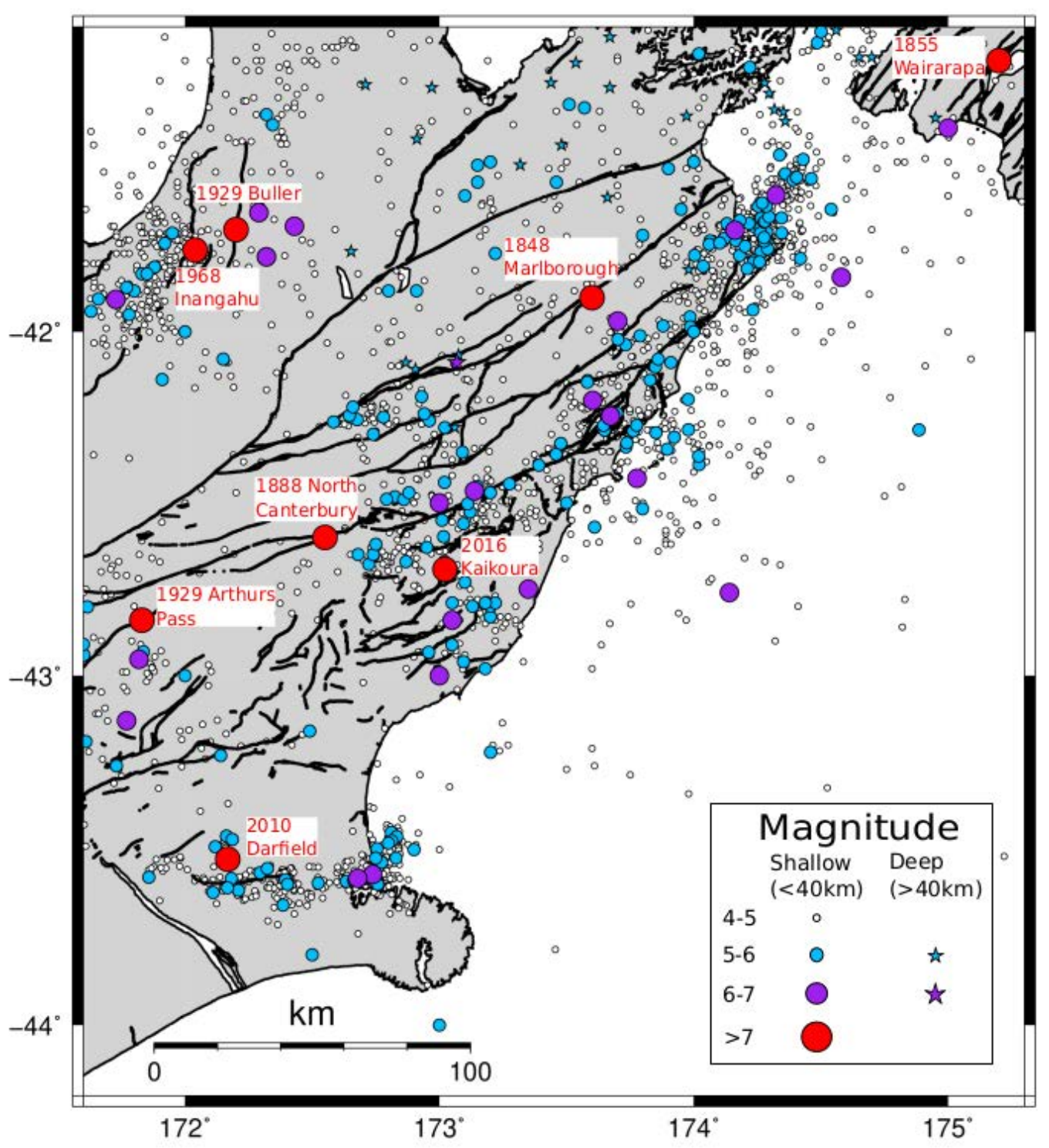

Figure 1.3: Historical Earthquake Map from 1840 to 2018. Showing earthquakes greater than magnitude 4 for shallow earthquakes $(<40 \mathrm{~km})$ by circles and for stars for deeper earthquakes $(>40 \mathrm{~km})$. Earthquakes (circles and stars) are coloured by magnitude: $M_{w}$ 4-5 in white, $M_{w}$ 5-6 in blue, $M_{w}$ 6-7 in purple and $M_{w}>7$ in red. The large magnitude faults $\left(M_{w}>7\right)$ are all dated and named by location. The black lines show all active faults in the region. 


\subsection{Study Design}

The data has been recorded using a short-period network of 43 instruments. The deployment of these instruments began in 2009 and they are serviced by the University of Kyoto's Disaster Prevention Research Institute (DPRI). For the purpose of this study we have procured data for 19 stations as shown in Figure 1.4 for a seven-year period from the $1^{\text {st }}$ of January 2012 to the $28^{\text {th }}$ of February 2018. These 19 stations were chosen due to their proximity to the main faults that ruptured during the Kaikōura earthquake. This time period was also selected as it will contain nearly five years worth of data before the earthquake and just over a year of data after the earthquake.

To understand the seismic velocity changes before and after the 2016 Kaikōura earthquake two different techniques are applied. The first, follows the first primary objective and involves averaging the result from pairs of seismic stations that are a range of distances from the epicentre of the earthquake. The second technique follows the second primary objective and is used to understand how velocity changes are spatially distributed across the region. The region is split into quadrants to analyse different faults sections and different distances from the Kaikōura earthquake.

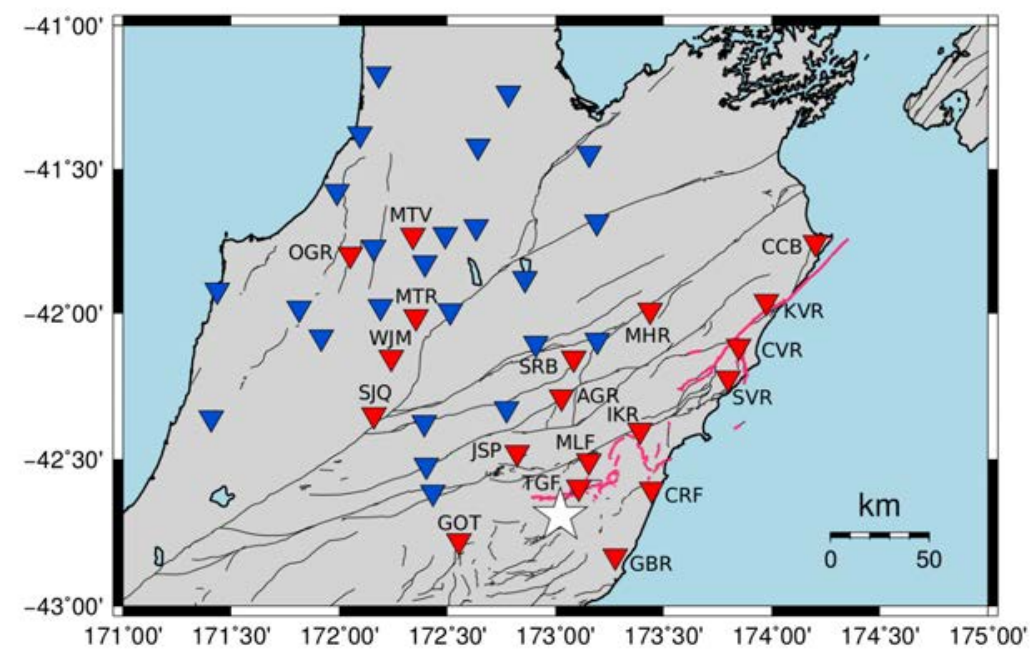

Figure 1.4: Map showing the location of stations used in this thesis. Red triangles show stations used in this thesis, while blue triangles show unused stations. The pink line show the faults that ruptured during the 2016 Kaikōura earthquake (white star) and the black lines show all active faults in the region. 


\subsection{Thesis Structure}

We begin by outlying the thesis structure by briefly describing the contents of each chapter (Section 1.5.1). Terms and acronyms used within this thesis are described in Tables 1.2 and 1.1 in Section 1.5.2. This thesis is structured as follows:

- Chapter 1 - Introduction: This chapter introduces the motivation and the objectives of this study. It also provides an introduction to the background area including geological setting and recent earthquakes that have occurred in the Kaikōura region.

- Chapter 2 - Background Theory: This chapter provides background on the theory of ambient seismic noise and how seismic velocity changes can be extracted. Within this chapter is also a description of ambient noise theory explaining Ambient Seismic Noise Properties, Cross-Correlation Techniques and Seismic Velocity Variations (Section 2.1) as well as how Ambient Noise can be used to detect changes from earthquakes (Section 2.2).

- Chapter 3 - Data: This chapter introduces the nineteen short-period, three component, seismic stations used in this thesis.

- Chapter 4 - Methods: This chapter describes the methods used to process cross-correlations for station pairs. This includes the introduction of the main software used, MSNoise (Section 4.1). Parameter testing was exceedingly important for this dataset. It was performed during this stage of the project and is discussed in length in the final section (Section 4.2).

- Chapter 5 - Results: This chapter presents the results after processing the cross-component station pair ambient noise through to velocity changes. First, we describe the stability of the velocity changes for different cross-component pairs (Section 5.1) Secondly, we describe the results of the two-year test period for the different cross-component pairs (Section 5.2). Once satisfied with the two-year results, the data period was lengthened to seven years. The results for the different cross-components are shown in Section 5.3. For both Section 
5.2 and 5.3, different stations will either be excluded or included depending on quality and duration, and this will be discussed in both sections.

- Chapter 6 - Discussion: This chapter has the discussion split into three parts. The first section investigates the depth sensitivity of the results using filter analysis and depth sensitivity kernels, and also analyses how distributed the results are across the northern South Island (Section 6.1). The next section discusses the overall result of the 2016 Kaikōura earthquake and if any changes were discerned from the 2013 Cook Strait earthquake sequence and from two large earthquakes in 2015 and early 2016 (Section 6.2). Mechanisms that could have caused the velocity changes in association with the Kaikōura earthquake will also be discussed within this section. In Section 6.3, previous studies will be used to compare to our velocity change results and determine whether processes suggested from previous studies can be invoked here. Finally in Section 6.4, we conclude by discussing the potential for real-time monitoring and how ambient noise can be used to better understand geologic hazard.

- Chapter 7 - Conclusion: The final chapter presents a summary of the major findings of this thesis and how this addresses the primary objectives (in Section 1.2). This chapter is concluded with recommendations for future work.

- Appendices: Appendix A shows data availability for different components and which stations were not used at all. Appendix B shows all of the final processing parameters and filters used in this thesis.

\subsubsection{Glossary of Acronyms and Terms}

In seismic ambient noise literature, different acronyms and terms have been used. In order to maintain consistency, two tables have been generated listing the different descriptors used in this thesis. Table 1.1 gives a list of acronyms and their meanings and Table 1.2 provides a brief description of different terms. 


\begin{tabular}{|l|l|}
\hline Acronym & Definition \\
\hline CC & Correlation Coefficient \\
\hline CCF & Cross-correlation Function \\
\hline DTT & Delay time against lag time \\
\hline DVV & Velocity variation \\
\hline EE & East-East component \\
\hline EN & East-North component \\
\hline EZ & East-Vertical component \\
\hline FFT & Fast Fourier Transform \\
\hline MFS & Marlborough Fault System \\
\hline MWCS & Moving-Window Cross-Spectral \\
\hline NE & North-East component \\
\hline NN & North-North component \\
\hline NZ & North-Vertical component \\
\hline PGA & Peak Ground Acceleration \\
\hline PGV & Peak Ground Velocity \\
\hline PWS & Phase Weighted Stack \\
\hline RMS & Root-Mean-Square \\
\hline SAC & Seismic Analysis Code \\
\hline SNR & Signal-to-Noise Ratio \\
\hline WLS & Weighted Linear Regression \\
\hline ZE & Vertical-East component \\
\hline ZN & Vertical-North component \\
\hline ZZ & Vertical-Vertical component \\
\hline
\end{tabular}

Table 1.1: Glossary of Acronyms 


\begin{tabular}{|c|c|}
\hline Term & Definition \\
\hline Ambient Noise & ield that contains both natural and cultural noise. \\
\hline Auto correlation & $\begin{array}{l}\text { Cross correlating a time series with the same component } \\
\text { from the same seismometer, e.g. when processing single } \\
\text { stations, EE, NN, ZZ. }\end{array}$ \\
\hline Component pair & $\begin{array}{l}\text { Where two different seismometer's components that have } \\
\text { been cross-correlated with each other. For example, com- } \\
\text { ponent pair ZE is a cross-correlation between a vertical } \\
\text { component from the first station and an east component } \\
\text { from the second station. }\end{array}$ \\
\hline $\begin{array}{l}\text { Cross- } \\
\text { component }\end{array}$ & $\begin{array}{l}\text { Cross correlating the components from a single station or } \\
\text { from station pairs. Cross-components of single stations } \\
\text { can only produce the following correlations: EN, EZ, or } \\
\text { NZ, whereas station pairs can also produce: EE, NN, ZZ } \\
\text { correlations across different stations. }\end{array}$ \\
\hline $\begin{array}{l}\text { Cross- } \\
\text { correlation } \\
\text { Function }\end{array}$ & $\begin{array}{l}\text { A measure of similarity between two time series (either } \\
\text { from the same or different stations and components) as } \\
\text { a function of lag time. }\end{array}$ \\
\hline Curre & $\begin{array}{l}\text { The CCF that will be compared against the reference } \\
\text { stack during the MWCS approach. The duration of the } \\
\text { current stack must be smaller than the reference stack. }\end{array}$ \\
\hline Lag ti & $\begin{array}{l}\text { Measure in the time domain of the relative offset between } \\
\text { two cross-correlation functions. }\end{array}$ \\
\hline stack & $\begin{array}{l}\text { The background CCF compared to the current stack } \\
\text { in the MWCS approach. The duration of the reference } \\
\text { stack must be larger than the current stack. }\end{array}$ \\
\hline Single- & $\begin{array}{l}\text { Either a cross correlation (e.g. ZZ) or an auto correla- } \\
\text { tion (e.g. EZ) where only components from the same } \\
\text { seismometer are correlated. }\end{array}$ \\
\hline Station pair & $\begin{array}{l}\text { Two seismometers where the components are cross- } \\
\text { correlated with each other. For example, IKR-MLF, } \\
\text { where IKR is the first station and MLF is the second } \\
\text { station. }\end{array}$ \\
\hline
\end{tabular}

Table 1.2: Glossary of Terms 


\section{Chapter 2}

\section{Background Theory}

In this chapter a review of the theory of ambient noise and its application to earthquakes is presented. The first section (Section 2.1) introduces various aspects of ambient noise theory. The second and final section (Section 2.2) discusses the application to this project and how this tool can be used to determine changes in seismic velocity before and after earthquakes. The introduction section for each chapter adds any additional theoretical information as required.

\subsection{Ambient Noise Theory}

Traditionally, seismic analysis has been based on direct waves. These are waves that have been emitted from seismic sources such as earthquakes or explosions. As a result of the emphasis on direct waves, background noise has mostly been unexamined. Seismometers were able to record background noise but they didn't have enough data storage ability to save it, so until the twentieth century they only saved the parts they thought were important. Recent developments in acoustics and seismology suggest a relatively new method to measure the elastic response of the Earth, also known as ambient noise, by extracting the travelling waves from the diffuse or random wavefields (Shapiro and Campillo, 2004). This method then led to a rapid expansion into studying seismic ambient noise. In this section, surface waves and wavefield 
properties will be investigated, as well as how ambient noise methods will be used in this study. Green's functions will also be discussed as they can be estimated from seismic noise cross-correlations and this usage has evolved to be an important and widely used technique to determine seismic velocity and its changes (Groos et al., 2012; Weaver, 2005; Curtis et al., 2006).

\subsubsection{Ambient Seismic Noise Properties}

Small continuous movements, unrelated to earthquakes or explosions, led scientists in the nineteenth century to the conclusion that the Earth was moving (Milne, 1886; Gutenberg, 1958). Milne (1886) first mentions different types of earth movements and tries to categorize them into the following; either earthquakes (sudden violent movement of the ground), Earth tremors (small magnitude movements that escape detection), Earth pulsations (movements which are overlooked due to the length of their period), and lastly Earth oscillations (movements of long period and large amplitude). Later, Gutenberg (1958) discovered during a gravity survey that the Earth's surface undergoes small continuous movements unrelated to seismic or explosive activity. As more sensitive seismometers began to be introduced in the twentieth century, the general consensus begun to conclude that rather than the Earth's surface moving with only earthquakes or explosions, the surface was continuously moving (Gutenberg, 1951).

Seismic noise can be typically described as recorded seismic energy that is not of earthquake origin (Bonnefoy-Claudet et al., 2006). Energy sources that contribute to seismic noise include but are not limited to; human activity, solar and lunar tides within the Earth, fluctuations in temperature and atmospheric pressure, storms, and ocean waves (Stein and Wysession, 2009). The noise field is generally divided into two bands: cultural ( $<1$ second period) and natural ( $>1$ second period) ambient noise (Bonnefoy-Claudet et al., 2006). The interaction of ocean waves generates oceanic microseisms, which dominate the natural ambient noise spectrum at two different peaks. The word "microseisms" is commonly used to denote the continuous 
oscillations of the ground of periods between 3 and 10 seconds which are recorded by sensitive seismographs, and which are not due to earthquakes or to local causes such as rain, traffic or gusts of wind (Longuet-Higgins, 1950). The primary peak at $12-18$ seconds (approximately $0.07 \mathrm{~Hz}$ ) is caused by the interaction of waves on the coastline, whereas the secondary peak at approximately 6-9 seconds (approximately $0.14 \mathrm{~Hz}$ ) is thought to be generated by pressure on the seafloor from the non-linear interaction of ocean waves travelling in opposite directions, but with near identical frequencies (see Figure 2.1) (Longuet-Higgins, 1950; Peterson, 1993; Friedrich et al., 1998; Stutzmann et al., 2000; Larose et al., 2005; Shapiro et al., 2005; Bensen et al., 2007). These two peaks dominate the wave spectrum and, as they are consistent and persistent, they are often used in seismic wave studies (Bonnefoy-Claudet et al., 2006). Oceanic microseisms are always present but are more likely to dominate near coastlines (Stein and Wysession, 2009). The table below, (Table 2.1), is a summary of ambient noise sources according to frequency (Bonnefoy-Claudet et al., 2006). From Gutenberg (1958), Table 2.1 shows that in general, noise with high frequencies $(>1 \mathrm{~Hz})$ is dominated by cultural noise and noise with lower frequencies $(<1 \mathrm{~Hz})$ have natural origins.

\begin{tabular}{|l|l|l|}
\hline & $\begin{array}{l}\text { Gutenberg } \\
(1958)\end{array}$ & $\begin{array}{l}\text { Asten (1978); } \\
\text { Asten and Hen- } \\
\text { stridge (1984) }\end{array}$ \\
\hline $\begin{array}{l}\text { Oceanic waves striking along the } \\
\text { coasts }\end{array}$ & $0.05-0.1 \mathrm{~Hz}$ & $0.5-1.2 \mathrm{~Hz}$ \\
\hline $\begin{array}{l}\text { Monsoon/Large scale meteorologi- } \\
\text { cal perturbations }\end{array}$ & $0.1-0.25 \mathrm{~Hz}$ & $0.16-0.5 \mathrm{~Hz}$ \\
\hline Cyclones over the oceans & $0.3-1 \mathrm{~Hz}$ & $0.5-3 \mathrm{~Hz}$ \\
\hline $\begin{array}{l}\text { Local scale meteorological condi- } \\
\text { tions }\end{array}$ & $1.4-5 \mathrm{~Hz}$ & \\
\hline Volcanic tremor & $2-10 \mathrm{~Hz}$ & \\
\hline Urban & $1-100 \mathrm{~Hz}$ & $1.4-30 \mathrm{~Hz}$ \\
\hline
\end{tabular}

Table 2.1: Summary by Bonnefoy-Claudet et al. (2006) established from studies by Gutenberg (1958), Asten (1978) and Asten and Henstridge (1984).

The frequency distribution and amplitude of cultural noise (also referred to as artificial noise or human activity) can vary depending on the environment, but typically it produces noise at higher frequencies than $1 \mathrm{~Hz}$ (Gutenberg, 1958; Stein 

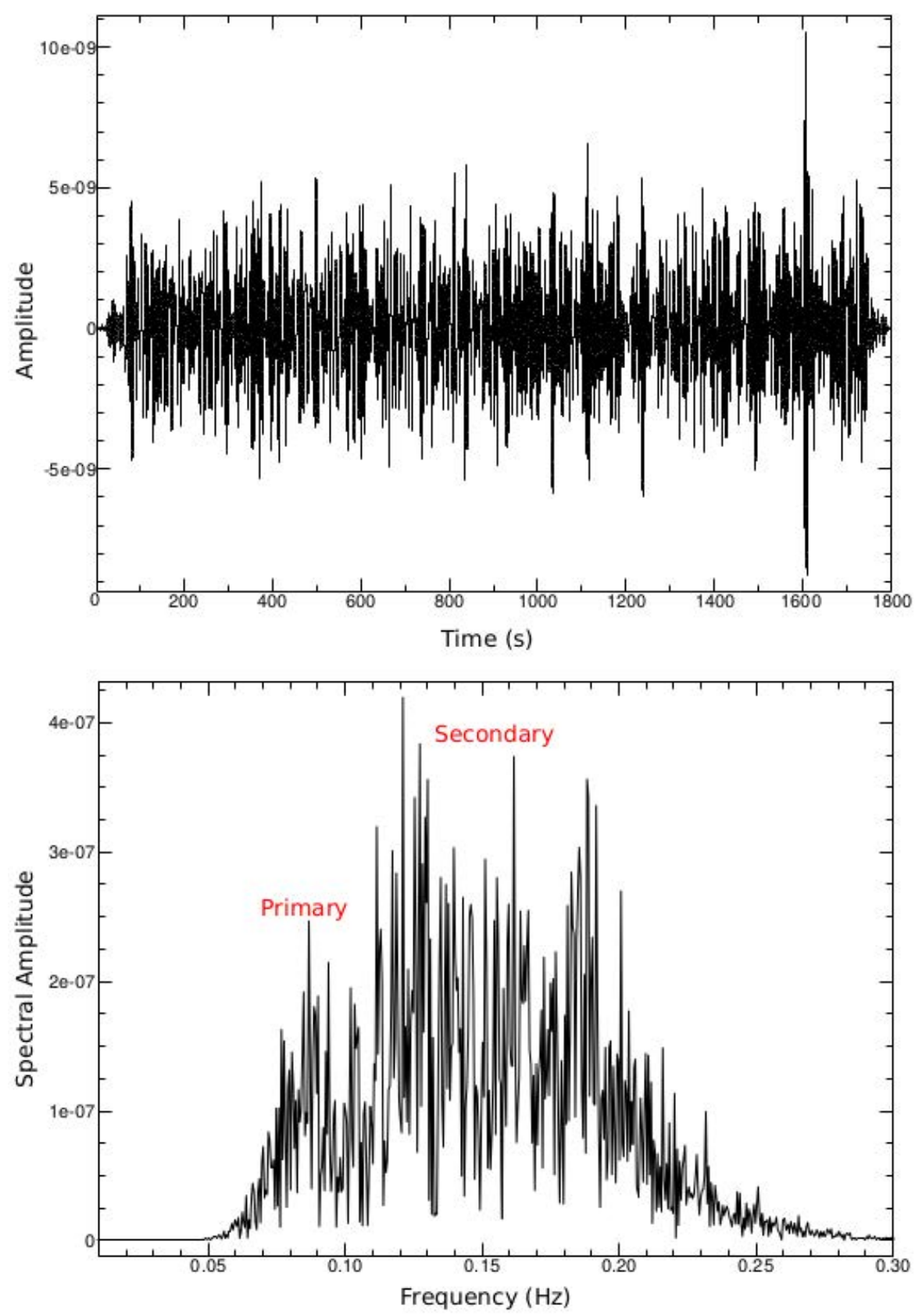

Figure 2.1: Primary and secondary microseisms in a continuous seismic recording. Example presented is for a 30 minute segment of a continuous seismic waveform bandpass filtered between 0.07 and $0.20 \mathrm{~Hz}$ for DPRI short-period station SVR on $15^{\text {th }}$ March 2017. The primary microseisms are visible at approximately $0.07 \mathrm{~Hz}$ and the secondary microseisms are visible at approximately $0.13 \mathrm{~Hz}$. The Earth hum, sometimes visible at $0.01 \mathrm{~Hz}$, does not appear in this waveform. 
and Wysession, 2009). At longer periods, the Earth's free oscillations are observable even in seismically inactive periods, and are known as the Earth's hum (Suda et al., 1998). The Earth's hum is generated by the interaction between the atmosphere, ocean and seafloor and probably through the conversion of storm energy to oceanic infragravity waves that interact with seafloor topography and atmospheric forcing (Rhie and Romanowicz, 2004).

As these factors are constantly at work, the Earth is continuously vibrating, giving seismometers noise to constantly record. One of the main ideas of using seismic waves is that the longer and further waves travel, the greater the variety of heterogeneities they encounter (Aki and Chouet, 1975). Therefore from the seismic coda, waves of local earthquakes will be backscattered waves from numerous randomly distributed heterogeneities (Aki, 1969). Coda waves were interpreted as scattered waves from the inhomogeneities within the Earth by Aki (1969, 1980).

There are two types of surface waves that propagate through the upper crust; Rayleigh and Love waves. Rayleigh (1885) first identified surface waves that are ellipticallypolarised waves. At the surface, the particle motion of Rayleigh waves are retrograde elliptical. With increasing depth the particle motion switches to prograde ellipse (Stein and Wysession, 2009). The apparent velocity of a Rayleigh wave, $L_{r}$ in a half-space is

$$
L_{r}=0.92 \beta,
$$

for a Poisson solid, where $\beta$ is the shear-wave velocity (Stein and Wysession, 2009). A Poisson solid is a material that assumes that the Lamé first parameter $(\lambda)$ is equal to the second Lamé parameter $(\mu)$, also known as the shear modulus. A material that approximates a Poisson solid is a good approximation for the Earth (Stein and Wysession, 2009).

Love (1927) further identified surface waves that are linearly polarised in the horizontal plane, called Love waves. The propagation velocity of Love waves also varies with frequency and wavelength. Seismic velocity increases as the depth of surface wave penetration increases. As Love waves require a velocity structure that varies with 
depth, they cannot exist in a half-space, unlike Rayleigh waves. Rayleigh waves are recorded on the vertical and radial traces and Love waves on the transverse. Rayleigh and Love waves can be identified in the correlations from their polarizations but not from their speeds as in different structures one can travel faster than the other and vice versa (Campillo, 2006).

Surface waves recorded by seismograms are the sum of harmonic waves with different velocities. At a given frequency, a single carrier wave travels at the phase velocity, $c$ which is given by

$$
c=\frac{\omega}{k}
$$

where $\omega$ is angular frequency and $k$ is wavenumber. The envelope, or beat pattern propagates at the group velocity, $U$, which is also the derivative of the phase velocity and is given by

$$
U=\frac{\partial \omega}{\partial k}
$$

Group and phase velocities are useful for ambient noise studies as their partial derivatives give their sensitivities to changes in shear velocities, and therefore material properties at specific periods as a function of depth (Aki and Richards, 2002; Stein and Wysession, 2009). Group and phase velocity relationships are important for ambient noise studies as they allow for surface waves to be inverted to calculate shear velocity maps (Lin et al., 2007; Stehly et al., 2009; Stein and Wysession, 2009).

\subsubsection{Cross-Correlation Technique and Green's Function Re- covery}

The ambient noise technique uses the theory that the cross-correlation of diffuse wavefields (e.g ambient noise, scattered coda waves) can provide an estimate of the impulse response, in these instances, an empirical Green's function, between them (Weaver and Lobkis, 2001; Derode et al., 2003; Snieder, 2004; Wapenaar, 2004; Larose et al., 2005; Bensen et al., 2007). An empirical Green's function 
represents the approximation of seismic response reconstructed between two stations by averaging the cross correlation of coda waves produced by distant earthquakes and recorded at those two stations (Shapiro et al., 2005; Campillo, 2006). Ambient noise methods usually use cross-correlations of naturally occurring microseisms at two stations to construct an empirical Green's function of the station path (Shapiro et al., 2005). Single stations cross-correlations and auto-correlations can also be used to construct an empirical Green's function. By definition, “a Green's function gives the displacement at point $\mathbf{x}$ that results from the unit force function applied at point xo" (Aki, 1957; Bakun and Bufe, 1975; Irikura, 1986; Shearer et al., 2006; Shearer, 2019).

These cross-correlation functions also give information about surface waves (Shapiro and Campillo, 2004). Empirical Green's functions were first generated from the seismic wavefield by Aki (1957). Aki (1957) measured surface wave velocities from the propagation of microtremors. Claerbout (1968) suggested that one side of an auto-correlation of the seismogram due to an impulsive source at depth represents the seismogram due to an impulsive source at the surface. It was not until several decades later in helioseismology studies that interest was again shown in the application of ambient noise cross-correlations. Toward the end of the twentieth century, Duvall Jr et al. (1993) showed that is was possible to extract travel-distance information from temporal cross-correlations on the Sun's surface. A few others Giles et al. (1997) and Rickett and Claerbout (2000) also performed cross-correlations on the Sun's surface, to look at the flow of gas on the surface and at time-distance curves respectively. Weaver and Lobkis (2001) and Lobkis and Weaver (2001) demonstrate that a diffuse acoustic field is shown to have correlations equal to the Green's function of a body in laboratory conditions. Early improvements and the new technique of using Green's function has led to the methods that will be used in this study.

The first applications to seismology were seen a couple of years after these studies, with cross-correlations of earthquake coda produced to give the surface wave component of the Greens tensor (Campillo and Paul, 2003). This discovery was rapidly surpassed 
with the suggestion from Shapiro and Campillo (2004) that ambient noise randomizes over time, so Green's functions can be generated from long seismic records without the need for large earthquakes (Sabra et al., 2005; Shapiro et al., 2005). Green's functions can be generated from a variety of distance scales; as a result, recent studies have focused on a wide range of study regions and scales (e.g. Brenguier et al., 2007; Bensen et al., 2007).

Initially, most ambient seismic noise studies focused on group velocities. Phase velocities were less commonly studied (Ritzwoller and Levshin, 1998) due to being dependent on the number of wave cycles travelled, whereas group velocities are independent of this. One of the first measurements of phase velocities was performed by Yao et al. (2006). Interstation Rayleigh wave (phase velocity) dispersion curves were obtained from empirical Green's functions derived from ambient noise interferometry and from traditional two-station analysis (Yao et al., 2006). From these methods Yao et al. (2006) were able to produce three-dimensional wave speed variations with uncertainties to study the structure from the crust to the upper mantle in the southeastern part of the Tibetan plateau, in the Sichuan and Yunnan provinces in China. Brenguier et al. (2007) managed to obtain dispersion curves and group velocities for each trace for different periods by reconstructing Rayleigh waves from cross-correlated data and then estimating the group velocity dispersion curve for each trace. From the two-dimensional group velocities a three-dimensional S-wave velocity model was generated. From this result, a high velocity body spatially delimited by the borders of the active caldera was clearly observed around the Piton de la Fournaise volcano (Brenguier et al., 2007). Stehly et al. (2009) used Rayleigh wave group velocity measurements to construct Rayleigh group velocity maps of the Alpine region, to study the crust in western Europe. The results were in good agreement with the result of numerous active experiments in the Alps and provide a continuous image of the Alpine structure (Stehly et al., 2009). Lin et al. (2012) used $\mathrm{H} / \mathrm{V}$ ratios, which are a measure of Rayleigh wave ellipticity, and phase velocities to develop crustal density and $V_{p} / V_{s}$ for the near surface. 
Observations of diffuse seismic wavefields (namely ambient noise and scattered coda waves) alleviate some of the problems affecting traditional surface wave measurements made on teleseismic earthquake recordings (Lin et al., 2007). Recent theoretical work has revealed that under the assumption that the sources of the ambient noise are evenly distributed; the Green's function between two points can be estimated from the cross-correlation of recordings made at two locations (see Figure 2.2) (Lin et al., 2007; Weaver and Lobkis, 2001; Derode et al., 2003; Snieder, 2004; Wapenaar, 2004; Larose et al., 2005).

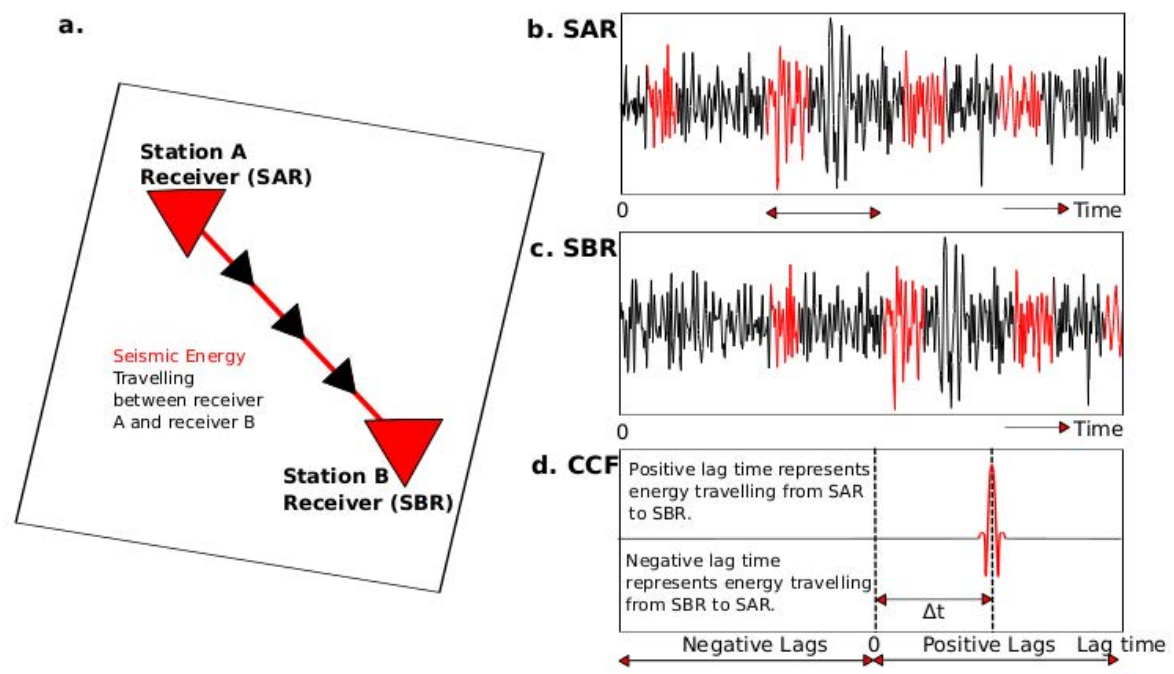

Figure 2.2: Illustration of the cross-correlation of seismic energy travelling between two receivers. (a) Seismic energy travelling on a direct path from Station A Receiver (SAR) to Station B Receiver (SBR). (b) Noise recorded by receiver A. (c) Noise recorded on receiver B. Similar energy is shown in red. (d) Cross correlating the two time series (A and B) can recover the travel time of the coherent energy in the form of a cross-correlation function (CCF). Adapted from (Yates, 2018).

It is possible to use both single station auto-correlations as well as cross-correlations. In terms of cross-correlations with different stations, the functions contain both positive and negative time lags, which are the same as causal and acausal Green's functions (Shapiro et al., 2006; Bensen et al., 2007). The positive time lags are the arrival times of the waves travelling in one direction as if one station is a point source and the other station is a receiver (Figure 2.3) (Campillo, 2006; Heckels et al., 2018). The negative time lag is the opposite, with the roles reversed. Previously mentioned studies have shown this result with both synthetic and real data and have given approximations to the Green's functions and retrieval of surface wave 
velocities (Brenguier et al., 2007; Stehly et al., 2009; Lin et al., 2012; Heckels et al., 2018).

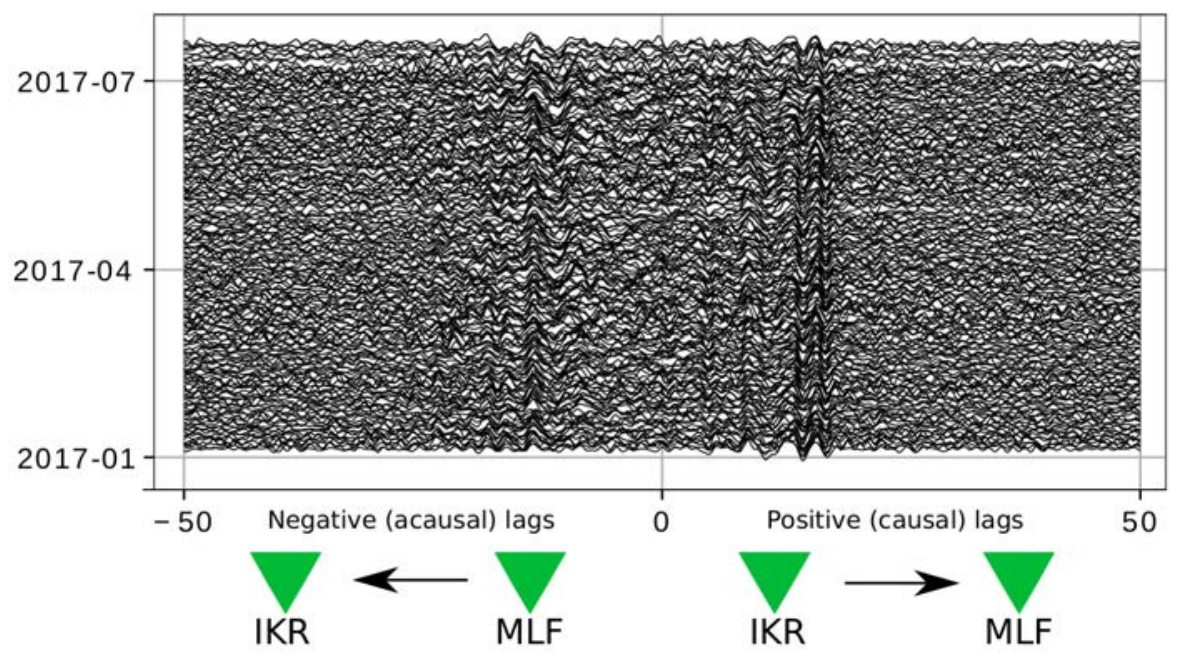

Figure 2.3: Positive and Negative time lags (causal and acausal respectively) for cross-correlation functions for station pair IKR-MLF for the beginning of 2017. Where each trace is a daily trace.

It is also possible to compute ambient noise cross-correlations using seismic energy recorded at only single-stations (e.g. Hobiger et al., 2014; Yates, 2018). Station-station ambient noise is calculated by the ambient noise that is received between stations. Single-station ambient noise is a signal that can be correlated with itself or other components in the same position (Hobiger et al., 2014). A single-station function does not record direct waves (those that travel from the source to the receiver) but only singly and multiply scattered waves (Hobiger et al., 2014). This is useful for monitoring velocity changes with time but it can't be used to determine where velocity changes are occurring like the station-station method. Monitoring of singlestations is possible by either correlating the station components with themselves to give auto-correlations or by cross correlating different components of the same station, single-station cross-component. Using a single seismic station, it is then possible to have six independent two-sided correlations using either auto-correlations (EE, NN, ZZ) or single-station cross-correlation (EN, NZ, EZ). Cross-correlations yield a total of $n(n-1) / 2$ possible station pairs, where $n$ is the number of stations (Bensen et al., 2007). This may also be done on nine different cross-components, leading to $9(n)(n-1) / 2$ values, where $n$ is the number of stations. 
Researchers have demonstrated that cross-correlations of passive seismic recordings between pairs of seismometers yield coherent signals (Campillo and Paul, 2003; Shapiro and Campillo, 2004). This principle has resulted in coherent signals being reconstructed from noise recordings in many different fields such as regional seismic studies (Shapiro et al., 2005; Sabra et al., 2005; Bensen et al., 2007) and exploration (Draganov et al., 2007) seismology, ocean acoustic waves (Roux et al., 2004), helioseismology (Rickett and Claerbout, 2000), ultrasound (Weaver and Lobkis, 2001), atmospheric infrasound (Haney et al., 2014), and studies of the cryosphere (Marsan et al., 2012).

\subsubsection{Seismic Velocity Variations}

For the purpose of this thesis, measuring seismic velocities from ambient noise is crucial. Seismic velocities are useful as they provide intrinsic information about the properties of the materials that the waves are travelling through. Scientists take advantage of the velocity's sensitivity to determine the seismic structure. Assuming the material within the earth is linearly elastic, and isotropic, elastic moduli, $\lambda, \mu$ (Lamè constants) and density $(\rho)$ can be used to determine compressional $\alpha$ and shear wave $\beta$ velocities as:

$$
\alpha=\sqrt{\frac{\lambda+2 \mu}{\rho}}, \beta=\sqrt{\frac{\mu}{\rho}}
$$

From these velocity equations we see that as the density $(\rho)$ changes, changes occur to both compressional and shear waves velocity changes. If the density is assumed to be constant, which could be assumed in a region with similar geology, changes in the elastic moduli $(\lambda$ and $\mu)$ will result in changes in seismic velocity. Traditional seismological methods, such as P-S wave travel times, $V_{P} / V_{S}$ ratios and anisotropy have been commonly used to investigate crustal changes. However, these methods are subject to earthquake location and origin time errors (Clarke et al., 2011).

Poupinet et al. (1984) introduced a method to calculate changes in seismic velocity 
using earthquake doublets from cross-correlations. An earthquake doublet is a pair of microearthquakes that have nearly identical waveforms and the same hypocentre and magnitude but occur at different times. Poupinet et al. (1984) measured the difference in arrival time for individual earthquake doublets. For a wave from the first event, the time $(t)$ used to travel a distance $(d)$ is equal to $d / v$ where $v$ is the average velocity (Ratdomopurbo and Poupinet, 1995). For the second event of the doublet, the travel time is now $t+\delta t$ for the same wave travelling the same path with a new velocity $v+\delta v$ is $(t+\delta t)=d /(v+\delta v)$, where $\delta t$ denotes the difference in travel time (Ratdomopurbo and Poupinet, 1995). If $(\delta v \delta t)$ is assumed to be negligible the last two equations can be combined to find the following:

$$
\frac{\delta t}{t}=-\frac{\delta v}{v}
$$

This relationship shows that the fractional change in velocity is equal to the slope of the time delay in the coda. The relative velocity change for the time period of a particular moving stack is then the negative of the relative arrival time change.

Figure 2.4 demonstrates how seismic velocity changes can be recovered from ambient noise cross-correlation functions following the method first established by Poupinet et al. (1984). To calculate changes in seismic velocity, current cross-correlation functions produced by a smaller noise record are compared with a reference crosscorrelation function which is produced using a larger noise record. The delay times are measured from the difference between the current and reference cross-correlation functions for both the direct and scattered arrivals. A linear slope can then be fitted using Equation 2.5. Having both direct and scattered arrivals improves the fit of the slope and therefore the sensitivity of the measurements. Also, as scattered arrivals have travelled greater distances, they are also more sensitive to temporal changes compared to direct arrivals.

The technique of Poupinet et al. (1984) was extended for ambient seismic noise and was successfully used to detect seismic velocity variations (Brenguier et al., 2008b; 
a.

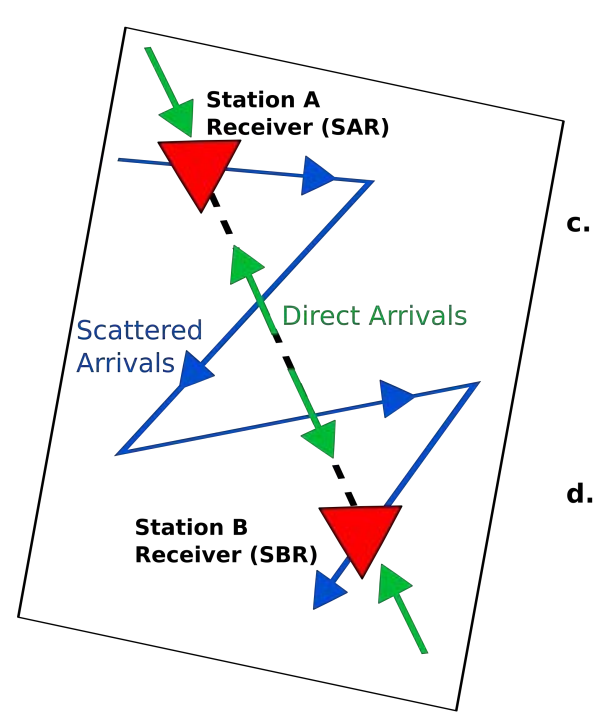

b. Reference CCF

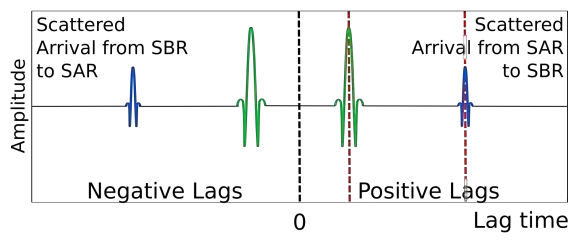

Current CCF after Velocity Decrease

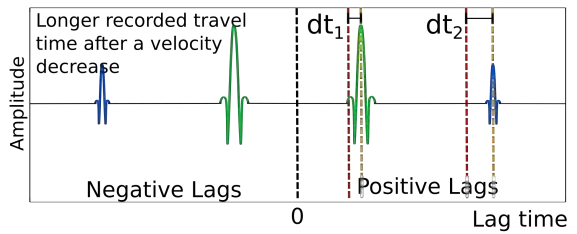

. Lag Time vs Delay Time

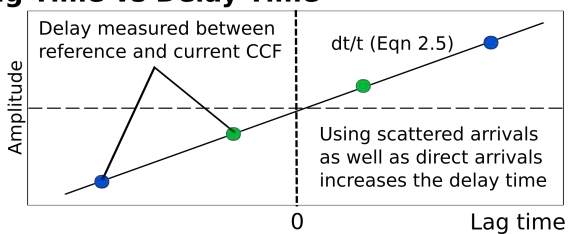

Figure 2.4: Illustration of how velocity changes are recovered from ambient noise CCF. (a) Direct and scattered arrivals between two receivers. (b) Reference CCF with waveforms showing direct and scattered arrivals for both positive and negative lag times. (c) Current CCF after a decrease in velocity for both direct and scattered arrivals. Difference in delay times between the reference and current $\mathrm{CCF}$ are shown by the dashed red (reference) and orange (current) lines. (d) Delay times are measured from the difference between the current and reference CCF for the direct and scattered arrivals. The linear slope is fitted using Equation 2.5. The velocity decrease is shown by the positive slope. Adapted from (Yates, 2018).

Wegler et al., 2009). Two processing techniques have been proposed in the literature to estimate relative velocity changes $d v / v$ in the coda of correlations. The first one, the doublet technique was first developed by Poupinet et al. (1984), also known as cross-spectral moving window technique (Frechet et al., 1989) or Moving-Window Cross Spectral (MWCS) (Ratdomopurbo and Poupinet, 1995). This technique works in the time-domain and in practice computes the phase shift between records for consecutive, overlapping time windows $\Delta t$ (Hadziioannou et al., 2011). For a given window $\Delta t$, the time shift $\delta t$ is assumed to be constant and is estimated in the frequency domain by measuring the Fourier cross-spectrum phase (Hadziioannou et al., 2011). For stations that recorded doublets, the MWCS technique was applied to compute the difference in P-wave arrival times. Earthquake doublets assume similarity between the two recorded waves, indicating that they are in close proximity to one another. It then follows that the distance between the two sources must be smaller than the source-receiver distance. Poupinet et al. (1984) showed that by 
using the MWCS technique they could detect temporal changes in the velocity of coda waves between two doublet families - co-located earthquakes - thus indicating that there were changes in the physical properties of the medium that the coda waves travelled through. Ambient noise with the doublet technique was shown to be advantageous by Sens-Schönfelder and Wegler (2006). They extracted scattered coda waves to obtain Green's functions from the cross-correlations. This technique is useful because, firstly ambient noise can be recorded continuously, and secondly, there will always be a difference in earthquake source mechanisms and locations, unlike scattered waves of ambient noise. The pair then continued to show a year later that this allowed for better recovery of Green's functions in the coda (Wegler and Sens-Schönfelder, 2007).

The second processing technique is in the time-domain and is discussed by Lobkis and Weaver (2003) and Sens-Schönfelder and Wegler (2006). It interpolates the coda at times $t(1-\epsilon)$ with various stretching factors $\epsilon$. This technique assumes a linear behaviour in $\delta t$ versus $t$, equivalent to a homogeneous relative velocity change $d v / v=\epsilon$ (Hadziioannou et al., 2011). Hadziioannou et al. (2009) showed in laboratory experiments that the stretching technique is more adapted to data with a low signal-to-noise ratio (SNR). In a recent study (Zhan et al., 2013) has demonstrated that the stretching technique can produce significant errors in its velocity measurements if the noise changes frequency content. This can be a problem for studies using a data of long duration as ambient noise changes in amplitude and frequency can occur over summer and winter months (Zhan et al., 2013). Due to this, the MWCS method was chosen for determining velocity changes.

Ambient noise studies typically produce very small velocity changes, on the order of $0.1 \%$ (Sens-Schönfelder and Wegler, 2011). Despite these small changes, seismic velocity changes can be recovered using ambient noise from many possible causes. A few of the major uses of ambient noise include measuring changes before, during and after earthquakes (e.g. Brenguier et al., 2008a; Minato et al., 2012; Taira et al., 2015; Heckels et al., 2018), volcanic activity (e.g. Brenguier et al., 2008b; Yates, 2018), 
and monitoring injection wells (e.g. Civilini, 2018). As ambient noise is so diverse, understanding the full process is important. With earthquakes, this allows us to understand more fully the seismic cycle as it increases the measurability of different velocity changes.

\subsection{Earthquakes and Ambient Noise}

Temporal changes of the crustal structure associated with seismic and volcanic activities are becoming an increasingly studied topic in Earth sciences, and have been investigated by many previous studies around the world. Other techniques have been applied to measure temporal changes, such as, determining changes that have resulted from earthquakes (Nishimura et al., 2000), anisotropy (Booth et al., 1990; Zatsepin and Crampin, 1997; Lev et al., 2006) and surface deformation (Han et al., 2006). Section 2.2.1 will discuss the physical process of temporal changes before and after earthquakes and Section 2.2.2 shows a few results from similar studies.

\subsubsection{Physical Process}

Seismic velocity changes after an earthquake can possibly occur due to damage in the fault zone rupture and the subsequent healing of cracks across fault planes (Heckels et al., 2018). This damage should be distinguishable by changes in features in the waveforms before and after significant earthquakes, such as the 2016 Kaikoura earthquake. Nur (1971) discovered in laboratory experiments that when hydrostatic pressure is raised, cracks close and the rock becomes stiffer. Tocher (1957) and Nur and Simmons (1969) observed in samples under uniaxial stress that the increase in velocity parallel to the applied stress axis was much greater than the increase in directions normal to the axis (Nur, 1971). This leads to the idea that at large pressures, such as from an earthquake on a fault plane, new cracks would appear which would result in a seismic velocity decrease (Nur and Simmons, 1969; Lockner et al., 1977). Tocher (1957) first suggests that observations of velocity could be used 
to determine stress changes in the shallow crust. These responses could potentially be strong along faults due to pre-existing microcracks. So by understanding the seismic velocities, it will be possible to get a better understanding of the microcracks and the damage affecting the rocks before and after the Kaikōura earthquake.

Several possible mechanisms for velocity changes associated with earthquakes have been proposed and are summarized by Xu and Song (2009). These possible mechanisms are 1) stress-induced changes of fault zone properties at seismogenic depth, 2) damage of the crust from fault zone rupture and subsequent healing, 3) damage of the shallow crust from strong-ground shaking, and 4) rapid changes in groundwater near the surface or fluid activities in the shallow crust (Xu and Song, 2009).

Wegler et al. (2009) explains how velocity changes can be affected by these mechanisms. Firstly, stress-induced changes can cause cracks to open and close, resulting in a velocity change (Poupinet et al., 1984; Taira et al., 2008; Brenguier et al., 2008a). Such changes will be deep, and positive and negative velocity changes are anticipated corresponding to regions of increased and decreased stress. Following the 2004 Parkfield earthquake, both Taira et al. (2008) and Brenguier et al. (2008a) determined that temporal changes were detected deeper than the subsurface and that modifications of the medium were likely due to changes in the stress field rather than cracks in the subsurface layer.

Secondly, physical damage to the fault zone caused by fault motion results in reduced velocity. Cracks open as a consequence of the main shock and close again as the cracks heal after an earthquake (Wegler et al., 2009; Li et al., 1998; Vidale and Li, 2003; Heckels et al., 2018). Heckels et al. (2018) inferred that this was the most likely mechanism for the $2010 M_{w} 7.1$ Darfield earthquake, as velocity changes were seen across the region and not only for station pairs crossing the Greendale Fault. The healing of microcracks as well as crack healing of the Greendale Fault was the dominant factor for velocity change.

Thirdly, near surface physical damage of the crust caused by nonlinearity of strong ground motion can cause velocity decreases (Wegler et al., 2009). Changes from this 
mechanism are expected to be shallow and to correlate with the strength of shaking (Rubinstein and Beroza, 2004). Shaking can be expressed by the peak ground velocities as these show where the greatest speed was reached by the ground. Rubinstein and Beroza (2004) measured the S-wave travel time of repeating earthquakes and detected a significant delay in the travel times at the stations that experienced the strongest ground motions from the $1989 M_{w} 6.9$ Loma Preita earthquake (Sawazaki et al., 2009).

Finally, pore pressure variations may cause changes in the hydrologic system. If the fraction of water in partially filled cracks changes, this can influence seismic velocity (Wegler et al., 2009). Earthquake-induced static and dynamic stresses and local hydrogeological factors can all influence groundwater level responses (Weaver et al., 2019). At the Merapi volcano, velocity variations show a strong seasonal influence and observations can be based solely on precipitation (Sens-Schönfelder and Wegler, 2006). These above four mechanisms can be considered to help determine the causes of velocity changes specially around the Kaikōura region as well as the impacts of large earthquakes on the surrounding region.

\subsubsection{Previous Studies}

Ambient noise experiments have been recognised for a while to be useful in examining velocity changes made by earthquakes around the world. This section will focus on previous studies that have observed either: a decrease or an increase in seismic velocity following an earthquake, a pre-earthquake seismic velocity change, or a velocity change as a result from monitoring either geothermal sites or hydrothermal systems.

Many studies have observed a decrease in seismic velocity as a result of an earthquake. Brenguier et al. (2008a) observed a seismic velocity decrease following two earthquakes, the $2003 M_{w} 6.5$ San Simeon and the $2004 M_{w} 6.0$ Parkfield earthquake. After San Simeon a decrease of $0.04 \%$ was observed and after Parkfield a decrease of 
$0.08 \%$ was observed. Seismic velocities remained low for three years after the Parkfield earthquake before slowly recovering to pre-earthquake values. Two possible mechanisms could be likely for the seismic decrease observed: 1) damage of the shallow layers by strong ground shaking, 2) coseismic stress change following postseismic relaxation (Brenguier et al., 2008a).

$\mathrm{Xu}$ and Song (2009) demonstrate how detecting temporal changes of the medium associated with major earthquakes has implications for understanding earthquake genesis. To achieve this, they reported on the temporal changes of surface waves following three major Sumatra earthquakes in $2004\left(M_{w} 9.0\right), 2005\left(M_{w} 8.6\right)$, and $2007\left(M_{w} 8.5\right)$. In this Sumatra study, ambient noise correlations were used to retrieve empirical Green's functions of surface waves between stations. Xu and Song (2009) show that the technique is powerful and is possible to detect temporal changes in the medium, with one station pair showing significant time shifts (up to 1.44s) after the 2004 and 2005 Sumatra events in Rayleigh waves at 10-20 s. By also determining the depth of sensitivity of their temporal changes from different periods of Rayleigh and Love waves, it was possible to determine that waves were sampling the upper-mid crust $(10-20 \mathrm{~km})$. As the temporal changes are too deep to have been caused by damage of the shallow crust or strong ground shaking and as the station pairs are far from the earthquake ruptured areas (separated by several hundred to 1,000 km) it is also unlikely that the temporal changes are associated with rupture damage of the crust or other properties of a fault zone. Therefore the most likely mechanism to have caused these changes is stress changes associated with major earthquakes and subsequent relaxation.

Minato et al. (2012) discovered a seismic velocity decrease of $1.5 \%$ using a six-month period centered on the $2011 M_{w} 9.0$ Tohoku earthquake. Two main aftershocks ( $M_{w} 5.8$ and $\left.M_{w} 6.7\right)$ were also included in this time period. The stations used were within a $100 \mathrm{~km}$ radius from the aftershocks and $\sim 250 \mathrm{~km}$ from the mainshock. Following the seismic velocity decrease, gradual healing occurred with seismic velocity values returning to $99 \%$ of the preseismic value a month after the Tohoku earthquake. 
The mechanisms most likely to account for these velocity changes are both from shallower, strong ground shaking, and deeper in the crust, coseismic stress release of the fault zone (Minato et al., 2012). Ambient noise cross-correlations were used by Froment et al. (2013) to monitor seismic wave speed variations associated with the $2008 M_{w} 7.9$ Wenchuan earthquake. Data was continuously recorded for two years by broadband stations in a region that covers the southern two thirds of the ruptured fault. The seismic velocity changes could not be explained by shallow perturbations of the crust as measurements have the strongest sensitivities between 10 and $20 \mathrm{~km}$ so are therefore related to deformation at depth in the crust.

Takano et al. (2014) examined seismic velocity changes due to the Earth tide by conducting cross-correlation function analysis of ambient seismic noise recorded at a small array in northeastern Japan. They found phase differences which are explained by seismic velocity changes of $-0.19 \pm 0.06 \%$ at $1-2 \mathrm{~Hz}$. These are interpreted to be caused by opening/closing of cracks or pores in the shallow subsurface due to the tidal strain (Takano et al., 2014). This seismic decrease was not caused by an earthquake but still demonstrates how changes in the subsurface can affect seismic velocities. Taira et al. (2015) perform ambient noise based monitoring to explore temporal variations of crustal seismic velocity associated with the $2014 M_{w} 6.0$ South Napa earthquake. They noticed a drop of $0.08 \%$ immediately after the South Napa earthquake. About $50 \%$ of the coseismic velocity drop was recovered in the first 50-day period after the South Napa mainshock. The stations used were a mixture of short-period geophones and broadband seismometers looking at the noise level in the frequency range $0.1-0.9 \mathrm{~Hz}$ (Taira et al., 2015). As the majority of the velocity recovery took place in the first 50 days, this suggests a healing process of the damaged rocks. The most likely mechanism for velocity change was damage of the crust from the fault zone rupture which then experienced subsequent healing.

Studies have also observed a gradual increase following rapid seismic decreases after earthquakes. This is mainly a result of a region experiencing damage of the crust in the fault zone as a result of the earthquake followed by subsequent healing which 
can occur on time frames of months to years (Brenguier et al., 2008a; Minato et al., 2012; Taira et al., 2015). Heckels et al. (2018) measured a velocity increase following the $2010 M_{w}$ 7.1 Darfield event in Canterbury, New Zealand. Continuous waveform data was collected surrounding the Greendale Fault for the four months following the earthquake. In this time a seismic velocity increase of $0.14 \pm 0.04 \%$ was determined near the Greendale Fault and interpreted as showing the post-seismic relaxation rates in the region (Heckels et al., 2018). Heckels et al. (2018) used the moving-window cross-spectral method to produce the seismic velocity increase and the result they observed suggests crack healing on the Greendale Fault.

Ambient noise methods can also be useful in monitoring geothermal sites. Obermann et al. (2015) used ambient seismic noise cross-correlations from nine stations to observe the loss of waveform coherence that could be horizontally and vertically constrained from the injection location in the fluid at St. Gallen geothermal site in Switzerland. Ambient seismic noise analysis was shown to assess the aseismic response of the subsurface and the reservoir dynamics (Obermann et al., 2015). Another recent study by Civilini et al. (2020) used ambient noise methods to monitor the subsurface deformation surrounding two producing geothermal fields (Rotokawa and Ngatamariki) in the Taupō Volcanic Zone. Seasonal and seismic responses were observed and it was shown that shear-wave velocity changes did not coincide with geothermal injection but did coincide with regional seismic and seasonal changes (Civilini et al., 2020).

Ambient noise interferometry was also used to understand more about the information about stresses present within hydrothermal and magmatic systems. This was done at White Island by Yates (2018) who used cross-correlations of station pairs and single stations to determine seismic velocity changes. Two periods of long-term velocity increase are detected at the volcano as well as two seismic velocity decreases of approximately $0.05-0.1 \%$ which are associated with a $M_{w} 5.2$ earthquake in 2008 and the $M_{w} 7.1$ East Cape earthquake in 2016 (Yates, 2018).

Using ambient noise methods to determine seismic velocities changes has been 
successful in locations around the world with stations that have been recording before and after large earthquakes. We hypothesize that using ambient noise methods would also show a decrease in seismic velocity following the $2016 M_{w} 7.8$ Kaikoura earthquake. This is most likely as many other studies (e.g. Brenguier et al., 2008a; Xu and Song, 2009; Minato et al., 2012; Froment et al., 2013; Taira et al., 2015) have also shown a velocity decrease following large magnitude earthquakes $\left(M_{w}>7\right)$. 


\section{Chapter 3}

\section{Data}

The dataset used in this thesis is comprised of nineteen short-period, three component, seismic stations which were deployed and are maintained by Kyoto and Tohoku University. The Japanese network is called DPRI after the Disaster Prevention Research Institute at Kyoto University and the data is archived in Japan (and we have full access to it - from the EQC proposal (Savage, 2018)). The short period network is used to establish 171 different station pairs with interstation separation distances ranging from $10.6 \mathrm{~km}$ (MLF-TGF) to the farthest distance of $181.3 \mathrm{~km}$ (CCB-SJQ) with an average distance of $79 \mathrm{~km}$ (Figure 3.1). All of these pairs are onshore with azimuth angles ranging from $20^{\circ}$ (SJQ-WJM) to $355^{\circ}$ (MTR-MTV). Six of the stations (CCB, CRF, CVR, KVR, GBR, and SVR) are quite close to the shore; between 2.4 and $7 \mathrm{~km}$ away with CRF being the closest. This is important as oceanic noise sources (such as wave energy) could affect our ambient noise results. Our target is to isolate changes that occurred as a result of the 2016 Kaikoura earthquake by averaging the results of all of these station pairs and to also identify spatial changes.

Stations were chosen by their location to the Kaikōura epicentre (shown by the white star in Figure 3.1, with surface faults also shown in this figure). Also to account for different faults, a few stations, OGR, MTV, MTR, WJM and SJQ were chosen on the western side of the South Island. Operation and quality of the seismic stations 


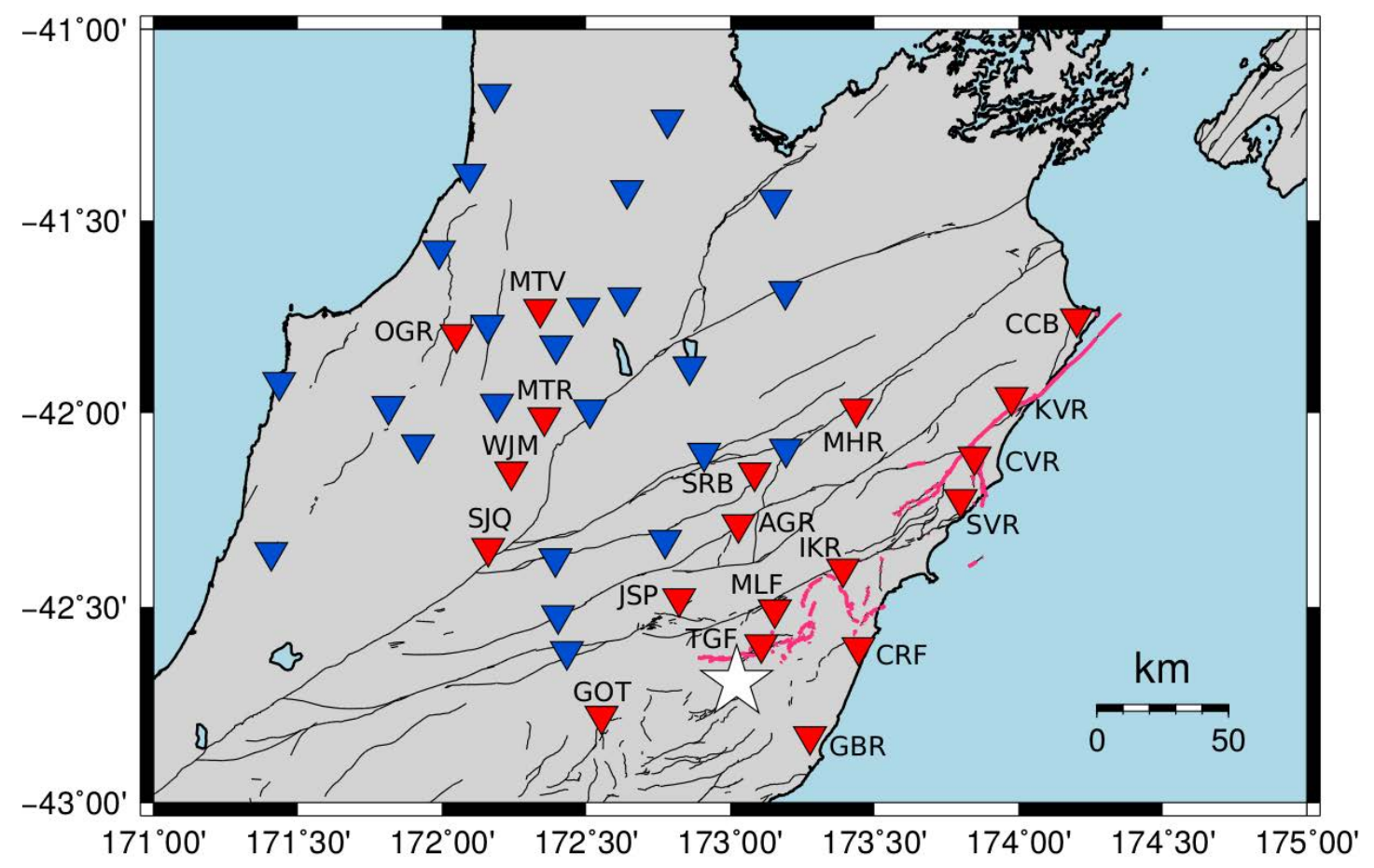

Figure 3.1: Map showing the location of stations used in this thesis. Red triangles show stations used in this thesis, while blue triangles show unused stations. The pink line show the faults that ruptured during the 2016 Kaikōura earthquake (white star) and the black lines show all active faults in the region.

vary throughout the data period (see Figure 3.2). The majority of the stations have been operating since 2012, with MTV being deployed the earliest, in 2009. Figure 3.2 shows this but does not include the two years where only MTV was operating. This figure also shows the reliable data with all of the bad waveforms being removed for each component. More information about unreliable stations can be found in Appendix A.

The velocity sensor that was used for the seismic data collection was a super-lowpowered KVS-300 velocity sensor (Kinkei System Corporation). This sensor is a moving coil type velocity transducer that measures velocity in three-components (Chiew et al., 2017). This sensor has a natural frequency of $2 \mathrm{~Hz}$. The seismic signals were recorded by data loggers (EDR-Z7000, Kinkei System Corporation) (Okada et al., 2019) with a sampling frequency of $1 \mathrm{kHz}$ and an analogue to digital resolution of 18 bits (Takano et al., 2014). An 8th order Butterworth anti-aliasing filter with a cut-off (-3 dB) frequency of $50 \mathrm{~Hz}$ for $250 \mathrm{~Hz}$ sampling was also applied. 
Data has been obtained for both the horizontal and the vertical components. However, quite a few of the stations had damaged components due to the 2016 Kaikōura earthquake or due to recording problems. As a result, a few of the stations have missing components for a large duration of the data period. This makes it slightly difficult to approximate the nine-component Green's function tensor, as a few components are missing (see Figure 3.2). Cross correlating across a wide range of station pairs improves the stability of measured velocity changes and hence the reliability of the results. 


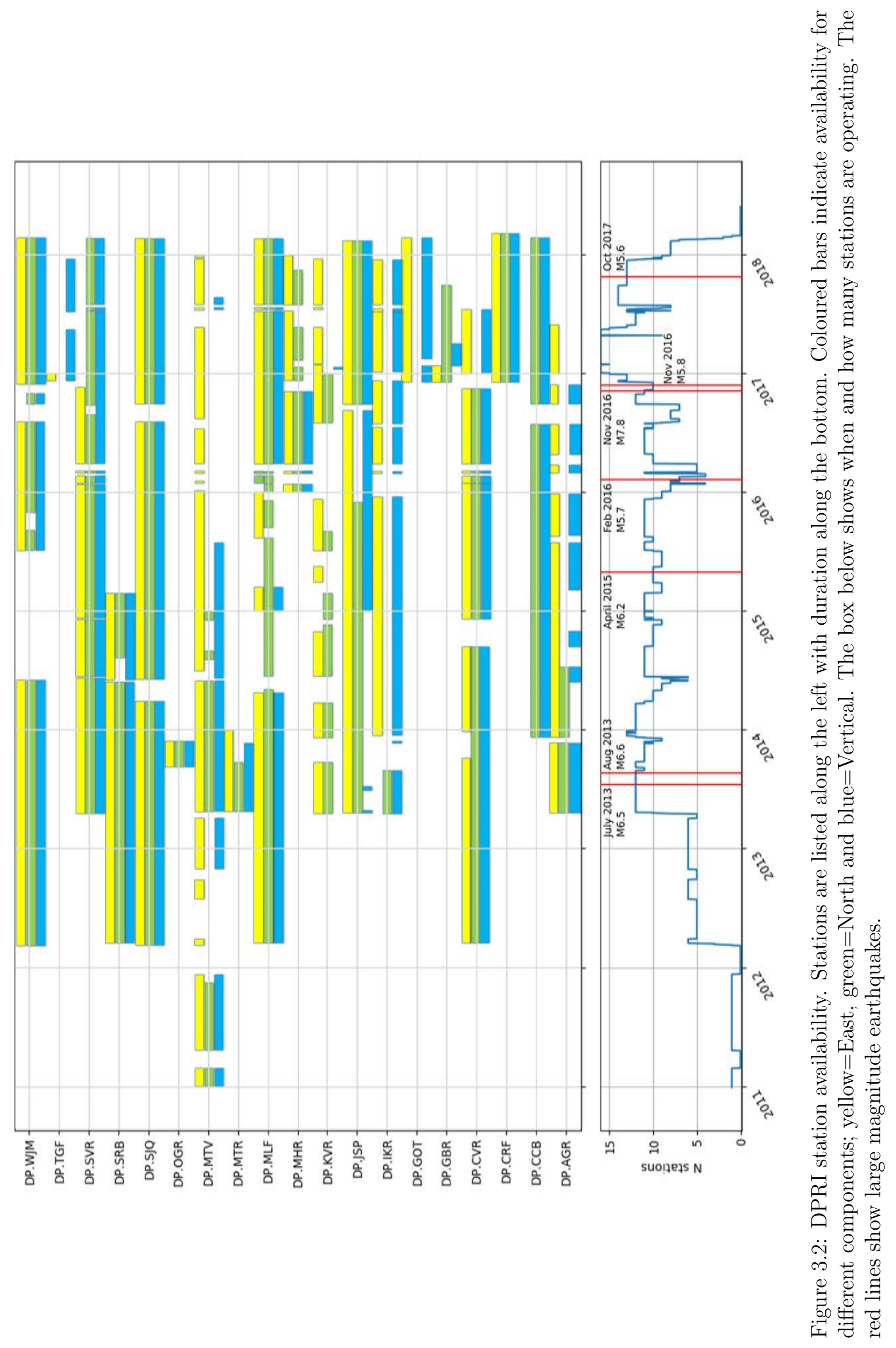




\section{Chapter 4}

\section{Methods}

This chapter introduces the processing sequence (Section 4.1) and describes the parameter justification. As the dataset was introduced in Chapter 3, this chapter will focus solely on the methods applied to this dataset. The parameter justification and the processing steps for the dataset are discussed in Section 4.2. This chapter explains the rationale behind the parameter choices in the aim to generate the most accurate method to use ambient noise to create velocity measurements.

\subsection{Processing Sequence}

Raw ambient noise data can be processed into velocity changes following four key processes. These processes commonly are: (1) pre-processing raw data, (2) computing cross-correlations, (3) stacking in the temporal domain and (4) determining velocity changes from relative travel time measurements. These steps are often used to generate travel time measurements, however different parameter choices are often made during each step. A Python package developed by Lecocq et al. (2014) called Monitoring using Seismic Noise (MSNoise) was used to process and recover velocity changes (version 1.5 for initial processing, version 1.6 for final parameter checks and running the full dataset). MSNoise starts with the raw data to produce travel time measurements as exportable data (Lecocq et al., 2014). Each of the aforementioned 
processes contain different choices available; these choices will be discussed in this section.

\subsubsection{Pre-processing}

Pre-processing of the raw data includes several important processes required prior to cross-correlation. The aim of these initial processing operations is to enhance ambient noise signals and suppress transient signals, such as earthquakes, to maximise the surface wave energy for estimated Green's functions (e.g. Heckels et al., 2018). Removal of any gaps in recordings and instrumental errors is also crucial. Preprocessing is important as it removes contamination that results in a non-diffuse wavefield that is not spatially uniform (Yao et al., 2006).

The initial raw input into MSNoise consists of continuous seismic day-long files that are named according to a known and recognised format by MSNoise. For the data in this thesis, the BUD format was chosen, so files were named by "Network/Station/Station.Network.Location.Channel.Year.Day". This network was named DPRI after the Disaster Prevention and Research Institute, which was installed and serviced by Tohoku and Kyoto University in Japan for 43 stations in the north of the South Island. Nineteen of these stations are used for this project. All of the data was merged into day long files, padded with zeros if no data existed, and these files were renamed appropriately for MSNoise. An instrument correction could be applied at this stage or within MSNoise, but we chose not to apply this correction as we are only working with the DPRI short-period stations. Pre-processing of the raw data includes several important steps required prior to cross-correlation. Firstly, raw continuous waveform data is downsampled, then the mean and trend are removed. As $2 \mathrm{~Hz}$ seismometers are being used, a broad bandpass filter $(0.01-2 \mathrm{~Hz})$ is initially applied to the cut day-long traces. Frequency normalisation and time-domain normalisation are applied. In both normalisations only amplitude information is altered, leaving phase data unchanged. They are both non-linear processes (Bensen et al., 2007) and so the order of operations is important. Finally, before the computation of the cross- 
correlation functions $(\mathrm{CCF})$, horizontal component waveforms are usually rotated from North and East orientations to produce radial and transverse components. For many stations, we did not have enough component data for each station to perform an accurate rotation (Figure 3.2), so they have been left as East, North, and Vertical components.

The parameters used for the pre-processing steps have to be considered carefully as they are heavily dependent on the data and the desired investigation. Bandpass filters and spectral normalisation filters depend upon the limits of the recording stations, the period band of interest and the spatial distribution of seismic stations (Poli et al., 2012).

Day long files were quality controlled assessed (i.e., no instrument problems apparent) using the Seismic Analysis Code processing system, or SAC (Helffrich et al., 2013) to make sure that different components were of similar amplitude and that there were no unnatural aspects to the waveform.

\section{Instrument Response}

An instrument response correction is required when multiple different networks with different seismometers are being used. Instrument responses are often neglected when all instruments are the same, to keep from introducing numerical artefacts in the response removal. As only the DPRI stations are being used, instrument response correction was deemed unnecessary. Other studies have also found this as Liu et al. (2014) also did not use an instrument response when determining seismic velocity changes from the 2008 Wenchuan earthquake.

\section{Time-domain normalisation}

Time-domain normalisation is performed on each of the day-long traces. This step suppresses earthquake signals and enhances the amplitude of scattered waves. This stage can also remove contamination from instrumental glitches. There are several 
methods that can be used to carry out time-domain normalisations. These vary with each dataset. The established and wide spread non-linear domain normalisations are the one-bit normalisations (e.g. Shapiro et al., 2005) and the running absolute mean (RAM) normalisation or root mean square (RMS) normalisation (Bensen et al., 2007).

RMS clipping reduces the dominance of earthquake signals in the resulting crosscorrelations by clipping amplitudes to a multiple of the signals RMS or standard deviation of a given day (e.g. Sabra et al., 2005; Roux, 2009). This is not ideal for the Kaikōura aftershock sequence, which includes a large magnitude event, with thousands of aftershocks which would increase the daily RMS amplitude, resulting in the smaller magnitude aftershock signals potentially not being removed.

One-bit normalisation is a much more aggressive approach (Bensen et al., 2007), whereby all positive amplitudes are given the value of 1 and all negative amplitudes are given the value of -1 , i.e.;

$$
\hat{x}_{i}= \begin{cases}1 & \text { for } x_{i}>0 \\ 0 & \text { for } x_{i}=0 \\ -1 & \text { for } x_{i}<0\end{cases}
$$

Although it is more aggressive, one-bit normalisation is the most common used method of temporal normalisation in ambient noise studies and is particularly important during aftershock sequences (e.g. Brenguier et al., 2007; Mordret et al., 2013; Savage et al., 2013). The following figure, Figure 4.1, shows the effect that temporal normalisation has in both the amplitude and the frequency spectrum. For the lower frequencies $(<2 \mathrm{~Hz})$ the amplitude is similar for all of the Fast Fourier Transforms (FFT's). For the higher frequencies $(>4 \mathrm{~Hz})$ the spike that occurred at $\sim 20-50 \mathrm{~s}$ in the original waveform is likely to be removed and is less visible in the frequency range of $4-10 \mathrm{~Hz}$ in the FFTs for the 1-bit normalised FFT than in the RMS-clipped FFT. 
a) Original Waveform

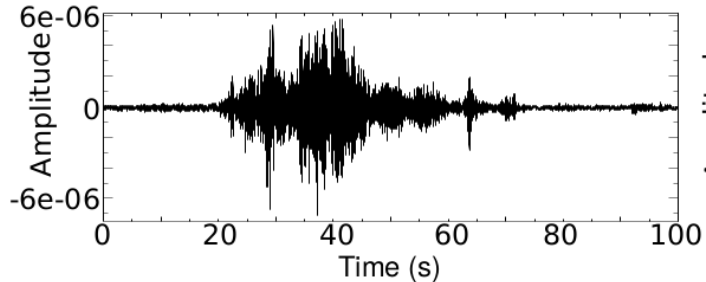

c) 1-bit Normalised

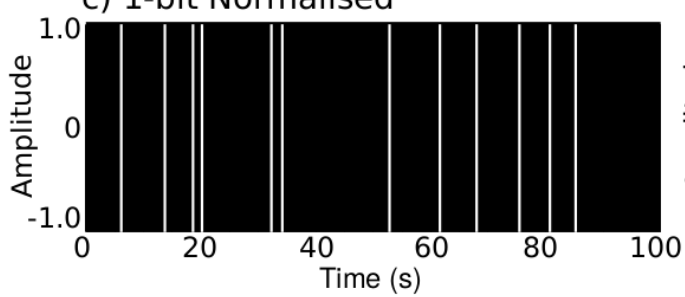

e) RMS-clipped

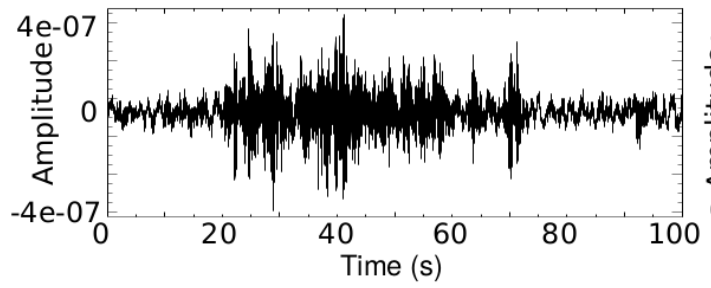

b) Original FFT

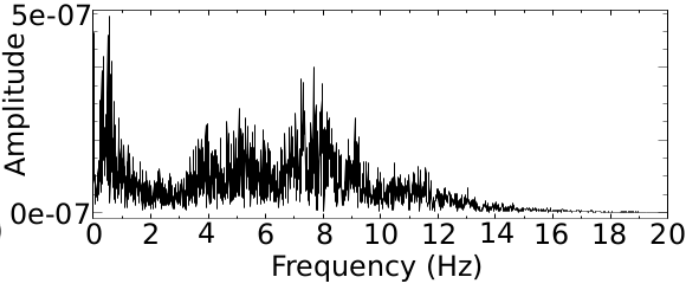

d) 1-bit Normalised FFT

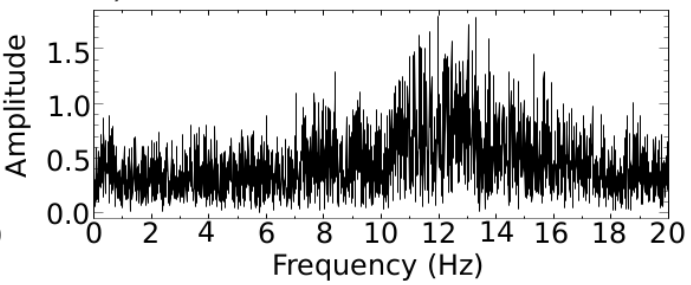

f) RMS-clipped FFT

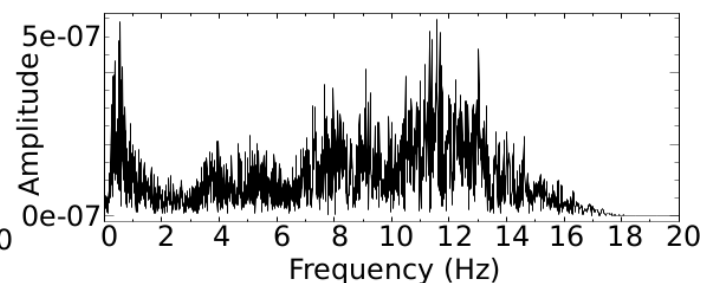

Figure 4.1: Different methods of temporal normalisation. (a) Original waveform, (b) FFT of original waveform, (c) 1-bit normalisation applied to waveform, (d) FFT of 1-bit normalised waveform, (e) RMS clipping applied to waveform, (f) FFT of RMS normalised waveform.

Some studies choose not to perform time-domain normalisation (e.g. Stehly et al., 2009), and chose instead to whiten the spectrum (see Spectral Whitening) of each record per day. However, for datasets containing high seismicity it is an extremely important pre-processing step (Bensen et al., 2007).

\section{Spectral Whitening}

Cross-correlations are often computed in the frequency domain: noise recordings are Fourier transformed and the spectrum at one station is multiplied by the complex conjugate of the spectrum at the other station (Weemstra et al., 2014). Spectral whitening involves an additional step where the amplitude of the obtained crossspectrum is set to unity for all frequencies (Weemstra et al., 2014). Frequency domain normalisation through spectral whitening acts to broaden the band of ambient noise signal in cross-correlations and also combats degradation caused by persistent monochromatic sources (Bensen et al., 2007). As monochromatic 
signals are suppressed and lower amplitude signals enhanced, this lowers the relative amplitude of the primary and secondary microseisms bands that occur at $7 \mathrm{~s}$ and $14 \mathrm{~s}$ (Longuet-Higgins, 1950; Groos et al., 2012). The resulting signal is smoothed and more equally distributed in the frequency spectrum. Spectral whitening is typically applied to a single time window cross-correlation function prior to stacking and in general, after time domain normalisation (Groos et al., 2012). In MSNoise, the traces are clipped using one-bit normalisation (time-domain normalized) and then whitened in the frequency domain (Lecocq et al., 2014). The intention is to improve the finally obtained stacked CCFs in terms of the representing broad-band Green's function estimates (Bensen et al., 2007). The approach of Spectral Whitening assumes that the wanted signal is present in the data but buried by uncorrelated noise.

\subsubsection{Cross-Correlation computation}

Following pre-processing the daily traces are cross-correlated. For each station pair where data is recorded for both stations on a given day, each component of one can be cross-correlated with the other. This produces nine different horizontal and vertical component combinations: EE, EZ, EN, NE, NN, NZ, ZE, ZN, ZZ, where E, N, and $\mathrm{Z}$ represent east, north, and vertical components respectively. The resulting signals have both positive and negative time lags. For the processing where we perform for positive time lags, the first letter is interpreted as the direction of the point source, and the second letter as the receiver response in that direction. For example, the EZ component gives the response to a point force in the E-direction at the first station recorded on the $\mathrm{Z}$ component at the second station. Negative time-lag signals reverse the roles of source and receiver at the first and second station (Figure 2.3). Based on reciprocity, symmetric components of cross-correlation functions are calculated by time reversing the negative lag signal and summing it with the positive one (Bensen et al., 2007). To produce daily cross-correlation functions, signals are cross-correlated in short time windows and then stacked.

The daily cross-correlation functions can then be stacked in two different ways. 
MSNoise provides two different stacking methods: linear mean or phase weighted stack (PWS). If the stack method is linear then a simple mean CCF of all windows is saved as the daily CCF. If the stack method is PWS then all of the PWS is computed and saved as the daily CCF (Lecocq, 2019). The PWS is done in two steps: first the mean coherence between instantaneous phases of all windows is calculated, and eventually serves a weighting factor in the mean (Schimmel and Paulssen, 1997; Lecocq, 2019). The smoothness of this weighting can be defined in MSNoise. PWS is good stacking option to determine the direct signal (sometimes called the "ballistic" signal) but not good for scattered waves that are used in the MWCS method. Because of this, linear stacking is the method chosen for the DPRI dataset.

\subsubsection{Moving and Reference Stacks}

Cross-correlation functions are stacked to improve the signal-to-noise ratio and therefore the temporal stability (Bensen et al., 2007). The purpose behind stacking is to achieve more coherent energy. When stacked, coherent energy sums constructively and incoherent energy sums destructively. Better coherency is often achieved with more days stacked together e.g. 30-days, however small velocity changes, especially if they occur over a short time frame, can be increasingly more difficult to recover with larger day stack sizes. The moving stack size should be large enough to calculate accurate velocity changes but small enough that changes aren't lost within the averaged period.

Choosing the reference stack length is an important parameter choice. The reference stack should represent the background state of the cross-correlation function. As such, the reference stack should always be longer (contain more days) than the current stack, for example, $N_{\text {Reference }}>>N_{\text {Current }}$ (Clarke et al., 2011). The main criteria for the size of both the reference and the current or moving stack is that the reference stack is representative of the background value whereas the current (or moving) stack contains information on the actual state of the crust (Clarke et al., 
2011). For this dataset, various moving stack choices are tested and the reference stack can either be set as the background state before the earthquake or could be for the entire duration, both of these options are tested in Section 4.2 and in the Results Chapter.

\subsubsection{Velocity Variations}

Relative velocity variations are computed by comparing the difference in arrival time between the current stack and the reference stack using the moving-window crossspectrum (MWCS) analysis method of Poupinet et al. (1984). This methodology was developed by Clarke et al. (2011). Delay times are measured in the frequency domain with a window defined in MSNoise. The length and overlap of the window chosen is crucial in the MWCS analysis (Clarke et al., 2011), both of which can be specified in MSNoise. Figure 4.2 demonstrates the resulting slope by using Equation 2.5 from Poupinet et al. (1984) and Ratdomopurbo and Poupinet (1995).

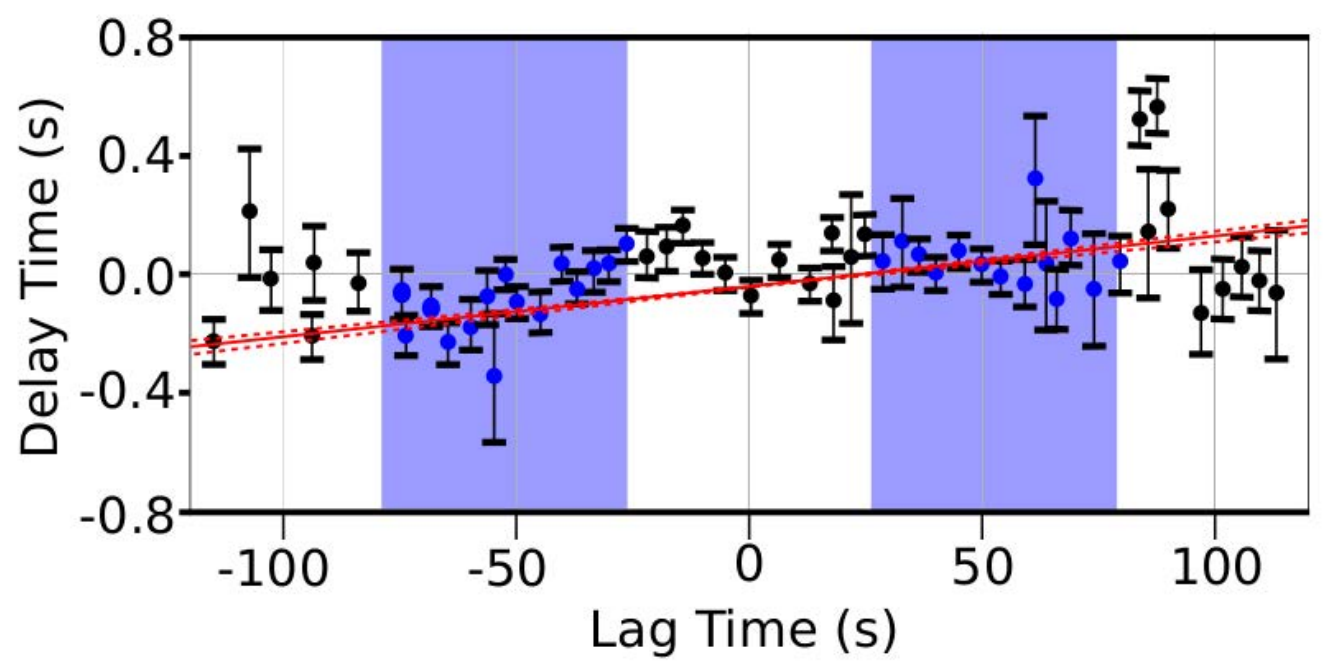

Figure 4.2: Moving-window cross-spectral analysis example, with a slope (red line) and its uncertainty (dashed red lines) fitted to delay values. Delay times used to calculate the slope are coloured blue while those that fall outside the windows are coloured black. Shaded blue boxes correspond to minimum and maximum lag times at positive and negative lags.

Following Clarke et al. (2011), time delays are computed using the MWCS method. The time delay measurements for both the current and the reference cross-correlation 
traces are then sliced in several overlapping windows (Lecocq, 2019). Each slice is mean adjusted and cosine tapered (1\% taper at both ends) before Fourier transformation to the frequency domain (Lecocq et al., 2014). The similarity between the two time series is assessed using the cross-coherence between energy densities in the frequency domain. The time delay between the two cross-correlations is found in the unwrapped phase of the cross spectrum and is linearly proportional to frequency (Lecocq et al., 2014). This "delay" for each window between two signals is the slope of a weighted linear regression (WLS) of the samples within the frequency band of interest (Lecocq et al., 2014). Velocity changes detected via ambient noise generally range between $0.01 \%$ to $1 \%$ (Hillers et al., 2015), so great care must be taken in pre-processing, station outage mitigation, and station pair selection, as just a few incorrect delay times can result in misleading velocity changes (Zhan et al., 2013; Daskalakis et al., 2016; Civilini, 2018).

In this stage, the coda windows (blue boxes in Figure 4.2) for the delay time measurements are also chosen in MSNoise. Minimum lag time and choosing the width of the window define the minimum and maximum values of the delay times that are used to calculate the slope of the delay times and from this, the change in velocity (Equation 2.5). This parameter is chosen to avoid direct arrivals, and as direct arrivals depend on inter-station distance, this parameter can also be set to be static or dynamic. This choice is discussed further along with other important parameter choices in Section 4.2.

\subsection{Parameter Justification}

This section aims to highlight and to ensure that the chosen parameters produce the most reliable velocity change values. Processing choices can hugely influence the cross-correlation functions recovered, so it is very important that all parameters are analysed and tested with different values. To determine the parameters we followed the method developed by Yates (2018). As more stations were added when they 
became available, this section will first discuss the parameters chosen with seven stations before expanding to the full nineteen station dataset.

\subsubsection{Initial Testing - seven stations, four months of data, $\mathrm{ZZ}$ component}

Ambient noise processing consists of many parameters, as a result many tests were undertaken to ensure that the most accurate parameters are chosen. To begin this process, a small initial sample set of data is used and most of the default MSNoise parameters are applied. These initial parameters are then modified as required as each processing sequence is worked through. Our initial dataset consists of two months of data before and after the Kaikōura earthquake from $1^{\text {st }}$ September 2016 to the $31^{\text {st }}$ of December 2016. Data was first bandpassed between 0.01-8.0 Hz, resampled to $20 \mathrm{~Hz}$, 1-bit normalized, and whitened between 0.02-7.9 Hz. Cross-correlations were then computed for 30 minute slices prior to performing a linear stack to give daily cross-correlation functions. These parameters were chosen as they were the default options for the processing software (Lecocq et al., 2014) and they appeared to be a good starting point for our dataset to begin iterating through different parameters. Also papers that have tried this method found good final results with parameters similar to these (e.g. Taira et al., 2015; Heckels, 2017; Yates et al., 2019). The final parameter choices are listed in Appendix B.

As the vertical-vertical component is often the most accurate, initial parameter testing will only use this cross-component to begin with for the following stations: CVR, IKR, KVR, MHR, MLF, MTV, and SVR. As initially we only had access to these seven stations for two years ( $1^{\text {st }}$ of January 2016 to $31^{\text {st }}$ December 2017) all of the stations were used instead of being more selective later in the parameter selection process when a larger duration and greater number of stations became available (shown in Figure 3.2). The unreliable data is not used in any of the analysis. This unreliable data is listed in Appendix A with the different components. 
The quality of data is quite poor for a few stations, with certain time periods for the initial seven stations as well as with the later addition of twelve more stations unusable. In order to prevent bias on the final result, it is crucial that only coherent data is used. The remaining incoherent data was decided based on a "common sense" approach and was not used. This approach included checking each month for each station across all components to determine if the station was recording properly. An example of this is if one component produced waveforms that did not detect earthquakes when other components did, the unresponsive component was removed from further analysis.

\subsubsection{Filter Frequencies and Coherent Noise}

The first thing that was investigated was producing a spectrogram to determine where in the frequency spectrum energy was arriving at the stations. From this a few different filters were composed while also keeping in mind that the seismometers are $2 \mathrm{~Hz}$ seismometers. In MSNoise there is a pre-processing bandpass filter as well as whitening and MWCS filters. All of these affect the signal in different ways and it is ideal if they all fit within each other such as shown in Figure 4.3.

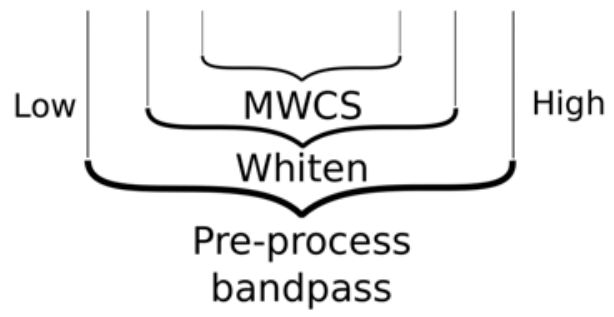

Figure 4.3: Different filters used as part of the processing sequence.

The spectrogram (Figure 4.4) shows that the largest filter, the pre-process bandpass filter, can go from $0.01 \mathrm{~Hz}$ up to $2 \mathrm{~Hz}$, and the whiten and MWCS filters could range from $0.1 \mathrm{~Hz}$ to $0.9-1.2 \mathrm{~Hz}$, as this is where the energy is. Figure 4.4 shows the full frequency scale with the high frequency earthquake signal and the low frequency background noise. The main noise from the earthquake, shown from 30390-30445 seconds (in Figure 4.4a) is of a higher frequency, on the order of $15-20 \mathrm{~Hz}$; this is 
a) Earthquake signal with background noise

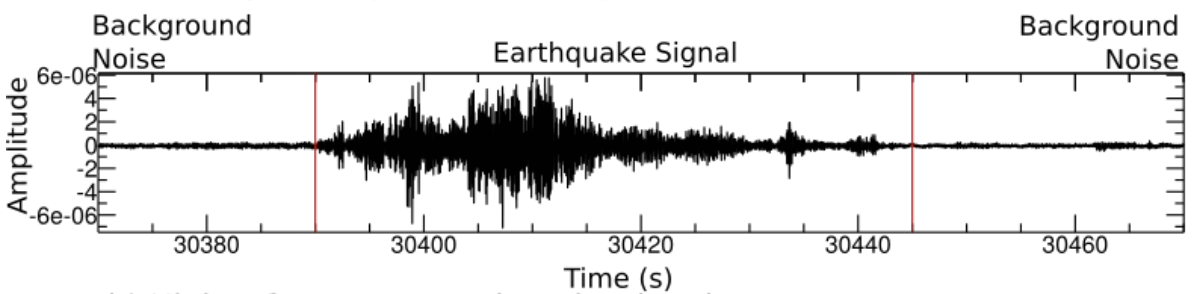

b) Higher frequency earthquake signal

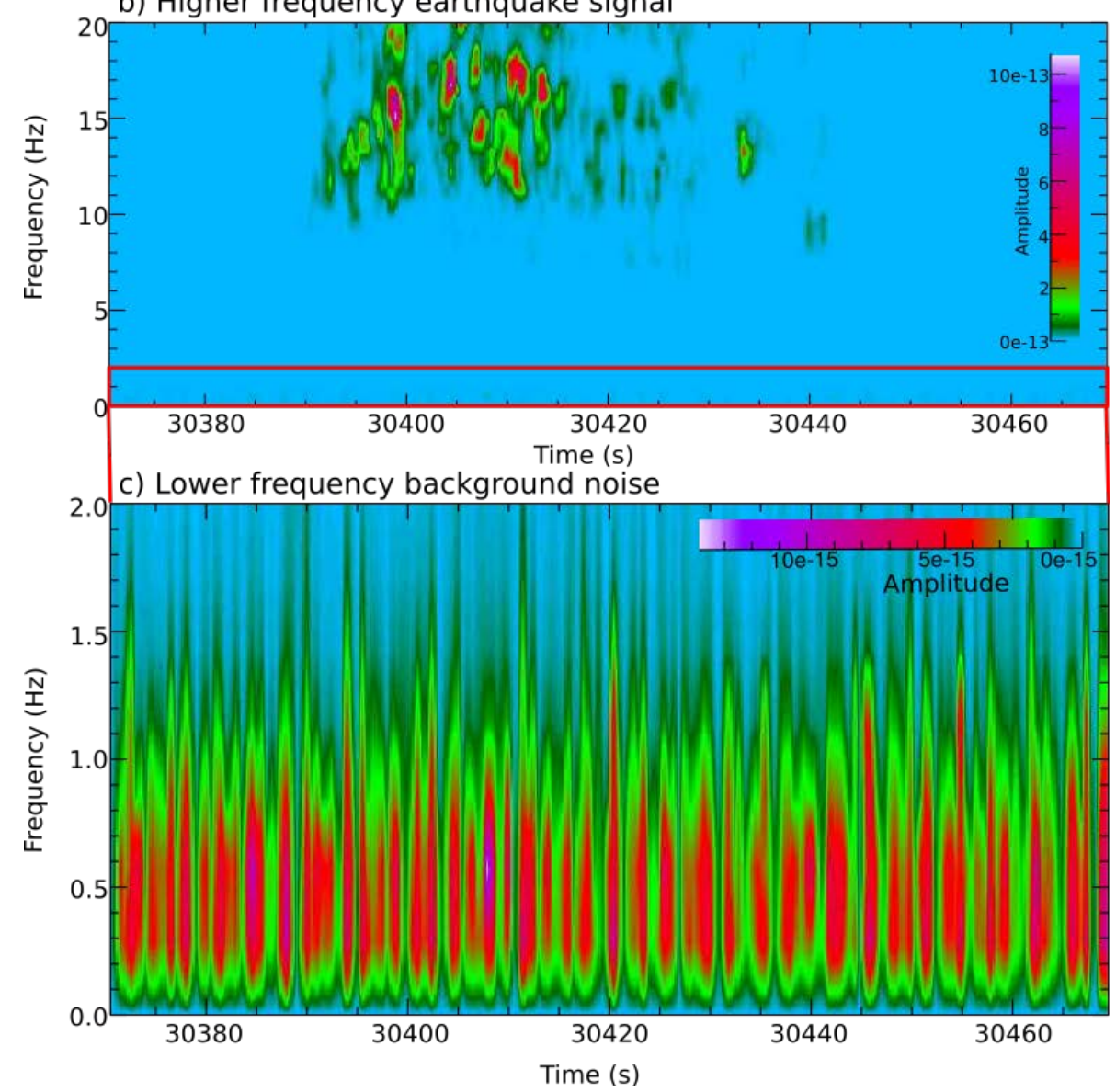

Figure 4.4: Spectrogram for station MLF showing energy frequencies. a) Earthquake waveform with the earthquake signal in the middle and background noise before and after. b) Spectrogram showing the frequency of the earthquake signal as well as faint background noise at low frequencies (see c)). c) Background noise. Energy primarily in frequency range $0.01-1.2 \mathrm{~Hz}$. Time in seconds since the beginning of the day for the $16^{\text {th }}$ of November 2016.

shown in Figure 4.4b. The background noise which is of interest is shown in Figure 4.4c. The background energy is present in the spectrogram both before, during and after the earthquake shown in the waveform.

Coherent energy can be used to determine where direct arrivals are occurring in the cross-correlation functions and to determine what frequency filters to use. If coherent energy is occurring, direct arrivals would have high relative amplitude. 
Lower amplitudes of coherent energy are recorded as the station-separation distances increases, since longer distances are less capable of recovering short-period energy (Sabra et al., 2005). For example, in Figure 4.5, the direct arrivals occur at later lag times as the inter-station distance increases.

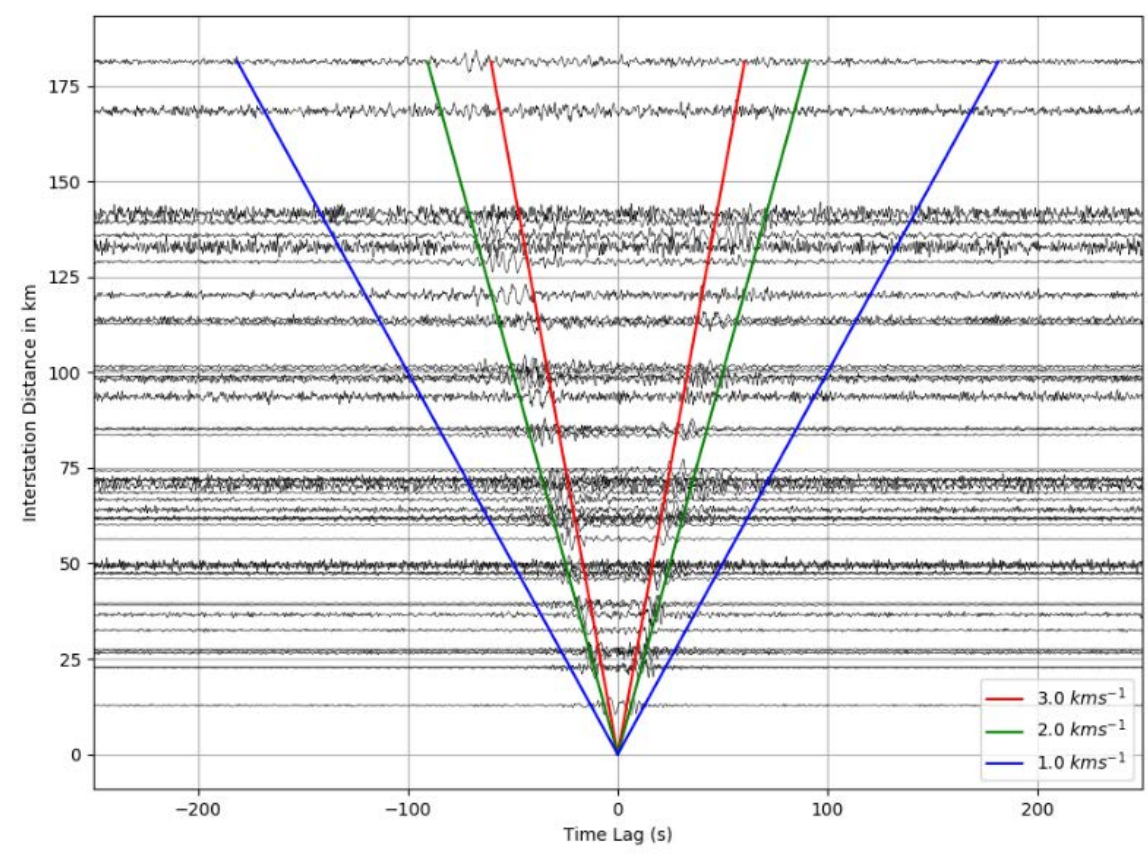

Figure 4.5: Distance against lag time plot for a $0.1-1.2 \mathrm{~Hz}$ filter showing different arrivals expected at different velocities for the vertical-vertical component.

\subsubsection{Parameter Processing with SNR}

Measuring the SNR of stacked sets of cross-correlation functions can be used to distinguish between stacks from which reliable delay-times can be measured, and from which they cannot (Clarke et al., 2011). Larose et al. (2007) notes that SNR decay seems to be driven by the decay of the Green's function. Clarke et al. (2011) uses the SNR method to investigate the stack size required for reliable delay times to be measured at Piton de la Fournaise volcano and also summarizes the method of Larose et al. (2007) as follows:

Firstly the level of noise $\sigma(N, t)$ in a stack is estimated to measure the variation between each cross-correlation function $(c c)$ at each lag time $(t)$ as follows: 


$$
\sigma(N, t)=\sqrt{\frac{\left\langle c c(t)^{2}\right\rangle-\left\langle c c(t)^{2}\right\rangle}{N-1}} .
$$

Here, $\langle$.$\rangle denotes the average over N$ single functions. Next, the level of signal, $s(N, t)$, is measured, in the stacked cross-correlation by taking its Hilbert envelope

$$
s(N, t)=|\langle c c(t)\rangle+i H(\langle c c(t)\rangle)|
$$

where $H($.$) denotes the Hilbert transform of the stacked function \langle c c(t)\rangle$ and $i$ is the imaginary unit. A Hilbert transform is a $\pi / 2$ phase shift, which can be viewed by thinking of the pulse as made up of sine and cosine functions and turning each into the other (Stein and Wysession, 2009). After $s(N, t)$ and $\sigma(N, t)$ are smoothed with a 30-second sliding Hanning window, the SNR of the stacked cross-correlation function can be estimated by:

$$
S N R(N, t)=\frac{s(N, t)}{\sigma(N, t)}
$$

Yates (2018) took the SNR process even further than Clarke et al. (2011) and used SNR to test many different parameters, such as filters, stacking methods, and length of cross-correlation slices. In this thesis, we follow Yates (2018) and also apply SNR to test many different processing parameters.

Firstly, SNR can be used to justify narrowing the bandpass and whitening bounds. Figure 4.6 demonstrates the usefulness of SNR as it shows that SNR increases when narrowing the default pre-process bandpass filter from $0.01-8 \mathrm{~Hz}$ to $0.01-4 \mathrm{~Hz}$. As the SNR has improved, the stability of the cross-correlation functions have also improved.

As Yates (2018) applied SNR to different parameters so shall we as shown in Figure 4.7. This figure demonstrates the testing process of key parameters by trying to maximise SNR. Testing includes: (a) different whiten filter choices, (b) whether to 


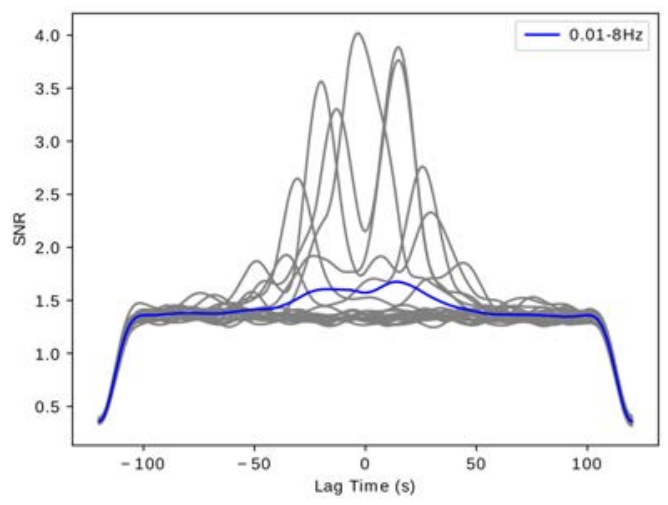

(a)

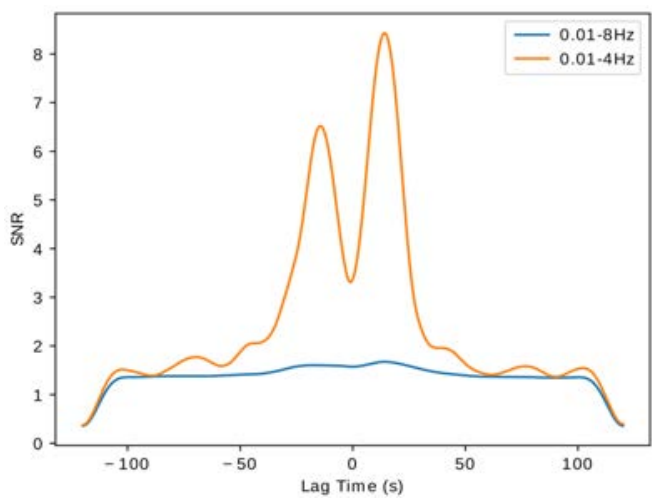

(b)

Figure 4.6: Initial pre-process bandpass filters that show when higher frequency noise is excluded the SNR value increases, therefore the stability of cross-correlation functions has improved. Light grey lines in a) are individual station pair traces whereas the blue line is the average of them all. This blue line in a) is shown again in b) where a lower filter $(0.01-4 \mathrm{~Hz})$ is used. b) is showing that using a filter from $0.01-4 \mathrm{~Hz}$ produces higher SNR values.

apply 1-bit normalisation or to clip waveforms at three times the RMS amplitude, (c) setting the power of the phase weighted stack (PWS) where $\kappa=0$ is linear, and $(\mathrm{d})$ choosing the length of the cross-correlation time slices computed prior to stacking. From preliminary tests a filter with a whiten value of $0.01-0.2 \mathrm{~Hz}$ was the most stable and produced the highest SNR value. In earlier tests it was determined that 1-bit normalisation improved the stability more than clipping the waveforms at three times the RMS amplitude. When comparing linear stacks to PWS's, stability was improved with a linear stack. This was also shown with the different plots of the PWS's, as linear $(\kappa=0)$ was the most stable. The most stable length currently found was $1800 \mathrm{~s}$ for the cross-correlation time slice. From these parameters test we found the SNR was maximised with the following process: using 1-bit normalisation, cross-correlating at 1800 second time slices and performing a linear stack while using seven stations and only testing four months worth of data.

\subsubsection{Initial Moving-Window-Cross-Spectral Parameters}

In this section, we highlight the main possible choices for reference stacks which are an important parameter for the moving-window cross-spectrum technique. For this 
dataset, three reference stack options are considered. These three options include: (1) a reference stack that only contains data prior to the mainshock on the $14^{\text {th }}$ of November 2016, for example, from $1^{\text {st }}$ of July 2016 to $1^{\text {st }}$ of October 2016 or (2) a reference stack that only contains data post the mainshock and majority of the aftershock sequence, for example, from the $1^{\text {st }}$ of July 2017 to the $1^{\text {st }}$ of October 2017 , or (3) a reference stack that includes all available data. By using a reference stack that is calculated from data either prior or a few years after the earthquake (to avoid the majority of the aftershocks and mainly contain background noise), this would be

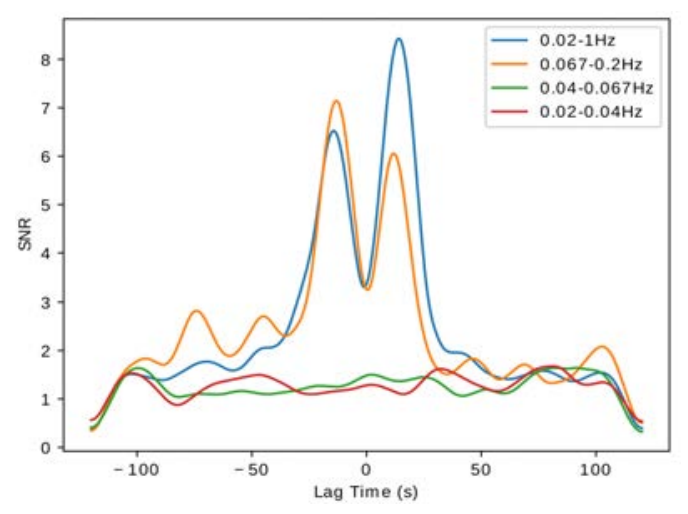

(a)

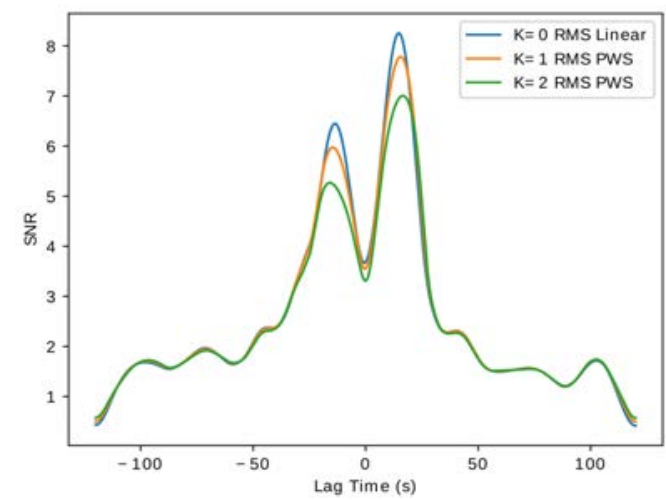

(c)

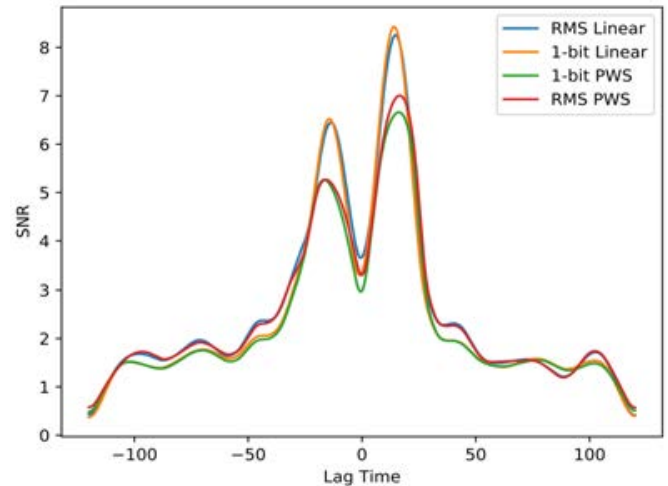

(b)

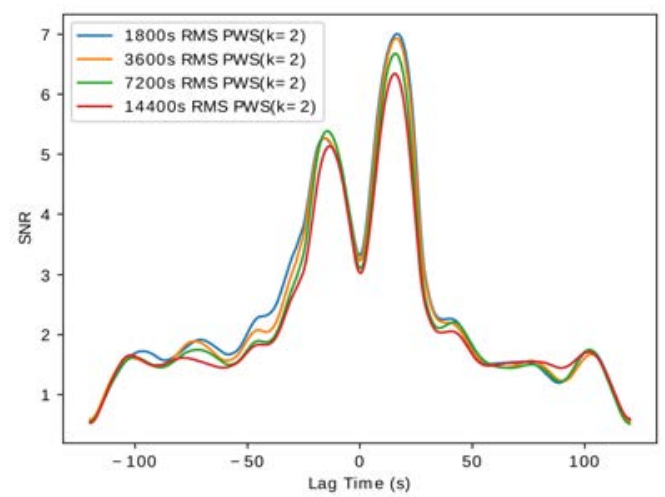

(d)

Figure 4.7: Different SNR parameter tests analysing filters, RMS vs linear, different phase weightings and cross-correlation time slice values. These SNR parameters are using seven stations and two months of data before and after the Kaikoura earthquake for the vertical-vertical cross-component. a) SNR test for different filters. The best results are for filters in the range 0.01-0.5 Hz and 0.067-0.2 Hz. Showing that higher frequency filters produce higher SNR values. b) RMS linear and 1-bit linear produce higher SNR values than phase weighted stack (PWS). Both RMS and 1-bit linear are quite similar in this example. c) RMS linear with a phase weighting of 0 produces the highest SNR. d). The lowest cross-correlation value of 1800 s produces the highest SNR. These figures show that currently the result that gives the highest SNR values is 1-bit, linear, $1800 \mathrm{~s}$ and using a high frequency filter in the range of 0.01-0.2 Hz. 
excluding any noise that would be heightened as a result of the $14^{\text {th }}$ of November 2016 earthquake. During periods with high activity such as aftershocks, the nonrepresentative cross-correlations are also at risk of contaminating the reference stack. Additionally, a dataset that spans the entire time period may not be entirely accurate if the velocity values are not at the same base level at the beginning and at the end of the period examined. The length of the moving-window and the step-size between successive windows are tested in conjunction with different filters and are finalised in Section 4.2.6.

\subsubsection{Testing with two years of data over seven stations - prior to adding all stations}

Upon expanding the dataset to two years, where previously the dataset was four months long, the majority of the processing parameters stayed the same. A 1-bit, linear normalisation was applied to $1800 \mathrm{~s}$ cross-correlated time slices for seven stations for the ZZ component. Different filter choices were also further analysed before 12 more stations were added.

\subsubsection{Testing with all of the stations for two years}

The full dataset (seven years with 19 stations) was made available once the owners (Kyoto University) received funding notifications of their own proposal in October 2019 and we, Victoria University of Wellington, had signed a letter of agreement about data sharing. As this was signed, we now have data for all of the stations near the Kaikōura earthquake from 2012-2018 (Savage, 2019). When this dataset was obtained, the first step was to determine which components had reliable data data that produced realistic records. Quite a few of the waveforms had bad data quality, such as undulating traces, very high or low amplitude compared to the other two components, and unrealistic spikes. To remove all of the bad days with poor data was a time consuming process as a few days in each month for each component 
and each station had to be checked. The final result of this is shown in Figure 3.2, showing that the availability of the station's components are quite varied and rather limited in the case of some stations. For example, OGR only had a few months of usable data and was not used in this thesis.

Once we had all of the data quality checked, tests were run for the ZZ component using 26 months of data ( $1^{\text {st }}$ of January 2016 to $28^{\text {th }}$ of February 2018) for as many stations as possible. These stations needed to have at least $\sim 75 \%$ of the data, as this would result in the stations containing data for both before and after the earthquake on the $14^{\text {th }}$ of November 2016. From previous tests, it had already been determined that 1-bit, linear normalisation would produce the best result as well as using a preprocess bandpass filter from 0.01-1.2 Hz (Figure 4.4) and whiten filters within the bandpass filter, such as $0.1-0.9 \mathrm{~Hz}$. The next step from here was to finalise a few more parameters:

- Correlation coefficiency plots to determine station pair accuracy,

- Minimum coherence value from SNR and coherency plots,

- Correlation duration time slice value,

- Delay time against lag time (DTT) windows from coherent energy and distance plots,

- Velocity variation (DVV) static or dynamic lag from coherent energy and distance plots,

- DVV unforced or forced plots,

- Reference stack duration.

One of the important steps in this process was computing correlation coefficient (CC) plots. These plot the average cross-correlation daily stacks for each different moving stack length against the reference stacks. The importance of this is that it shows if the cross-correlation stacks are working. If they are, they should produce a high correlation coefficient for that station pair for a particular cross-component. A "high 
correlation coefficient" value we determined was a value that was greater than 0.7 for the majority of the duration. This was quite a valuable step as it was useful in determining which stations data would be useful in this thesis. We used this to test the ZZ component with eight stations: CCB, CVR, IKR, JSP, MLF, SJQ, SVR and WJM. From this correlation coefficient test, we were able to determine that for all of the filters that we were testing, any pair that contained either CCB or CVR produced poor results due to low CC values for all moving windows. The result for the station pair CCB-CVR is shown in the following figure, Figure 4.8. As a result of the poor results from CCB and CVR, they were removed from any further processing of the ZZ component.

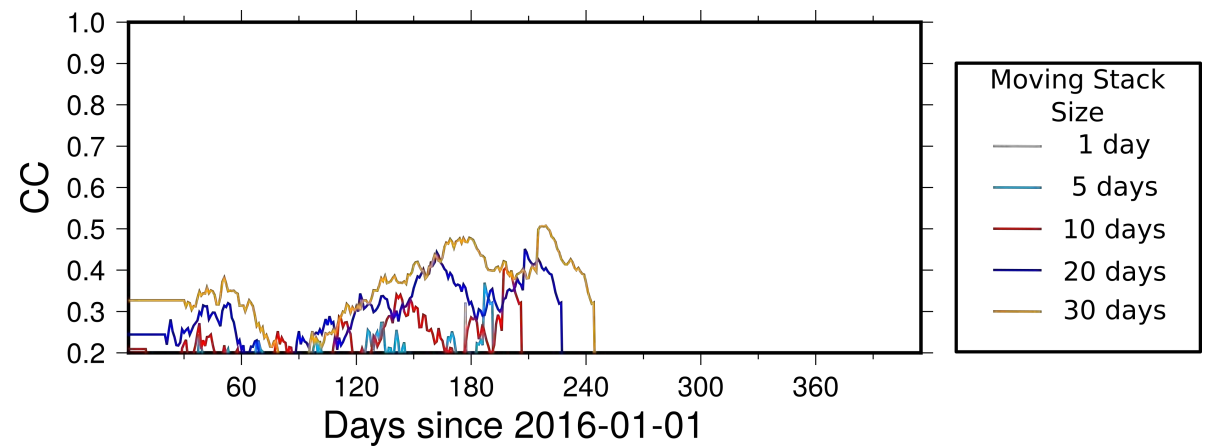

Figure 4.8: Correlation coefficient plot showing a poor result from station pair CCB-CVR with a $0.1-0.9 \mathrm{~Hz}$ filter. This was generated using the two-year test that started on the $1^{\text {st }}$ of January 2016 and ended on $24^{\text {th }}$ February 2017 due to data availability.

A good result of a cross-correlation is shown in Figure 4.9. For this station pair (SJQ-WJM) the different stack sizes consistently have high values. However at approximately day 240 there was missing data in both SJQ and WJM for the ZZ component, Figure 3.2, leading to lower $\mathrm{CC}$ values. Correlation coefficient plots are also useful as they show that the consistency in the stacking results does not increase greatly after the 20-day moving stack.

The correlation coefficient values decreased very slightly when the filter was changed from $0.1-0.9 \mathrm{~Hz}$ to $0.1-1.1 \mathrm{~Hz}$ (see Figure 4.10 to compare), irrespective of the MWCS window length and step. Only the filter frequency range had an effect on the correlation coefficient plots. This change is very minute but worth considering when determining what frequency ranges to use. 


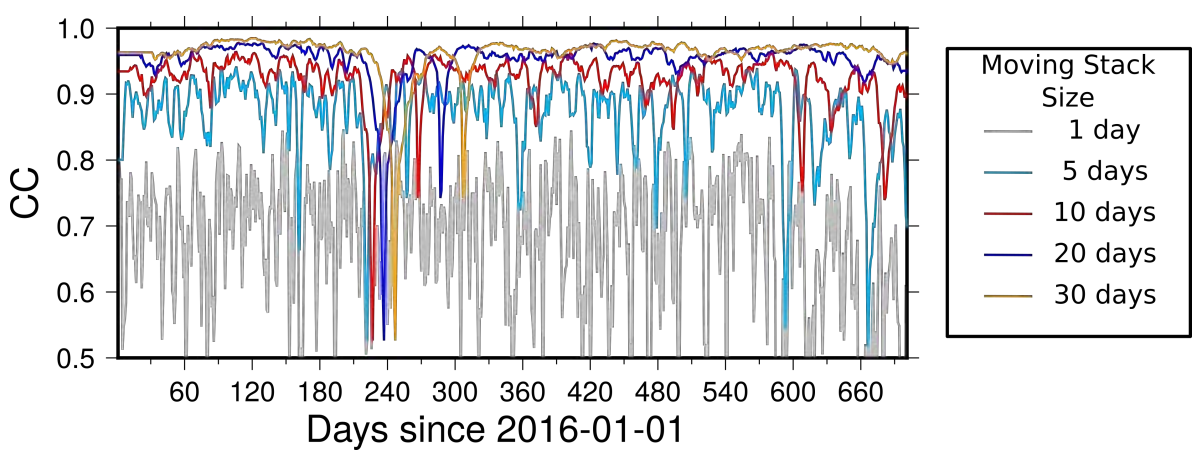

Figure 4.9: Correlation coefficient plot showing a good result from station pair SJQ-WJM with a $0.1-0.9 \mathrm{~Hz}$ filter. This was generated using the two-year test and started on the $1^{\text {st }}$ of January 2016 and ended on the $28^{\text {th }}$ of February 2018.

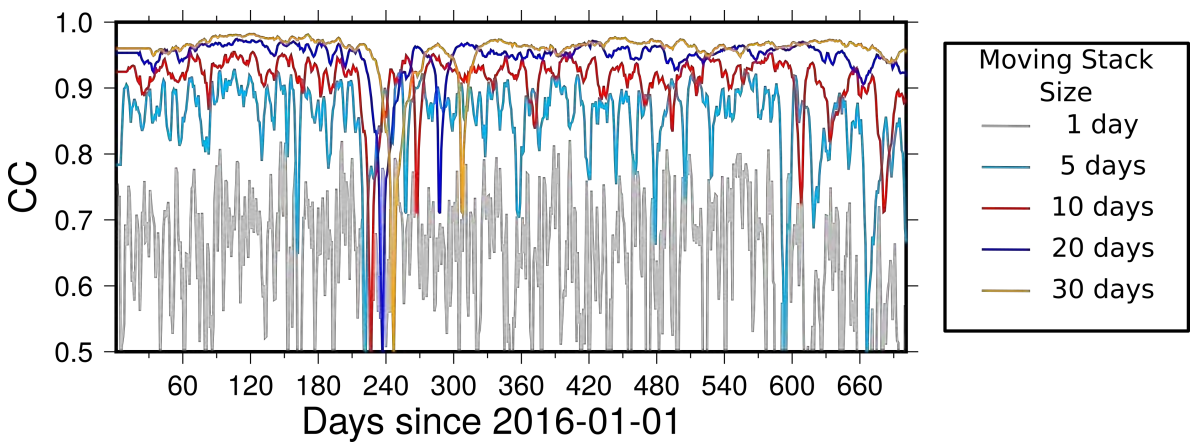

Figure 4.10: Correlation coefficient plot showing a good result from station pair SJQ-WJM with a $0.1-1.2 \mathrm{~Hz}$ filter. This was generated using the two-year test that started on the $1^{\text {st }}$ of January 2016 and ended on the $28^{\text {th }}$ of February 2018. This figure shows a very similar result to Figure 4.9 but a slight decrease of $\sim 0.03$ is visible across all moving windows.

The next step in the process was to test the SNR values, the coherency values and the SNR vs coherency for the different filters, with different MWCS window lengths and MWCS step lengths. In these tests we tested eight different filters (Table 4.1). The aim of this was to test several different filter values and to experiment with quite small MWCS window length and MWCS step values to produce more detailed time steps.

SNR tests proved quite useful (Section 4.2.3). From these more recent tests we were able to determine that using lower MWCS window length and MWCS step values, did not seem to affect the SNR or the coherency plots that much, but did affect the final velocity variation plots. As a result, all filters were run through all of the processing steps to determine how the final results changed between filters. The SNR and coherency results from using these filters (Table 4.1) are shown in Figures 4.11, 


\begin{tabular}{l|l|l|l|l} 
Filter number & Whiten Filter & MWCS Filter & $\begin{array}{l}\text { MWCS Win- } \\
\text { dow Length }\end{array}$ & MWCS Step \\
\hline 1 & $0.1-0.9 \mathrm{~Hz}$ & $0.15-0.85 \mathrm{~Hz}$ & $6 \mathrm{~s}$ & $2 \mathrm{~s}$ \\
2 & $0.1-0.9 \mathrm{~Hz}$ & $0.15-0.85 \mathrm{~Hz}$ & $10 \mathrm{~s}$ & $1 \mathrm{~s}$ \\
3 & $0.1-0.9 \mathrm{~Hz}$ & $0.15-0.85 \mathrm{~Hz}$ & $10 \mathrm{~s}$ & $2 \mathrm{~s}$ \\
4 & $0.1-0.9 \mathrm{~Hz}$ & $0.15-0.85 \mathrm{~Hz}$ & $16 \mathrm{~s}$ & $2 \mathrm{~s}$ \\
5 & $0.1-0.9 \mathrm{~Hz}$ & $0.15-0.85 \mathrm{~Hz}$ & $12 \mathrm{~s}$ & $2 \mathrm{~s}$ \\
6 & $0.1-0.9 \mathrm{~Hz}$ & $0.15-0.85 \mathrm{~Hz}$ & $12 \mathrm{~s}$ & $1 \mathrm{~s}$ \\
7 & $0.1-1.1 \mathrm{~Hz}$ & $0.15-1.05 \mathrm{~Hz}$ & $10 \mathrm{~s}$ & $1 \mathrm{~s}$ \\
8 & $0.1-1.2 \mathrm{~Hz}$ & $0.15-1.15 \mathrm{~Hz}$ & $10 \mathrm{~s}$ & $1 \mathrm{~s}$
\end{tabular}

Table 4.1: Table of filters used to test the ZZ component for the two-year dataset using reliable stations.

4.12 and 4.13. All of these plots are using the IKR-MLF pair for a 30-day stack size. This pair and stack size was chosen as it was the only combination that had data consistently for a 30-day time period. It was difficult to plot with a smaller stack size of 20-days as there were gaps in the data of at least 20-days.

In this SNR plot of the different filters (Figure 4.11) the best result is shown by the 0.1-0.9 Hz filter (filter number 2 in Table 4.1). This shows that as the frequency range increases the SNR values decrease slightly. Different MWCS window length and MWCS step filters were also plotted but even with different values than those shown, they plotted depending on their frequency range. In summary, the MWCS window length and MWCS step did not seem to be affected by SNR.

We then also calculated coherency values for the IKR-MLF pair with a 30-day stack size. This is shown against lag time in Figure 4.12 and against SNR in Figure 4.13. Against lagtime, (Figure 4.12) the highest coherency values are when the direct arrivals appear in the waveforms. This is to be as expected as they would have more coherent energy. To pick a good filter from the ones shown in the figure, the filter must show high coherence values for the entire lagtime period, from -120 seconds to +120 seconds, and not just when the direct arrivals arrive. From this figure (Figure 4.12 ) it is possible to see that the filter in red, $0.1-0.9 \mathrm{~Hz}$ with a MWCS window length of $16 \mathrm{~s}$ and a MWCS step of $2 \mathrm{~s}$ is clearly the best result, since it shows a high correlation value for the entire duration. 


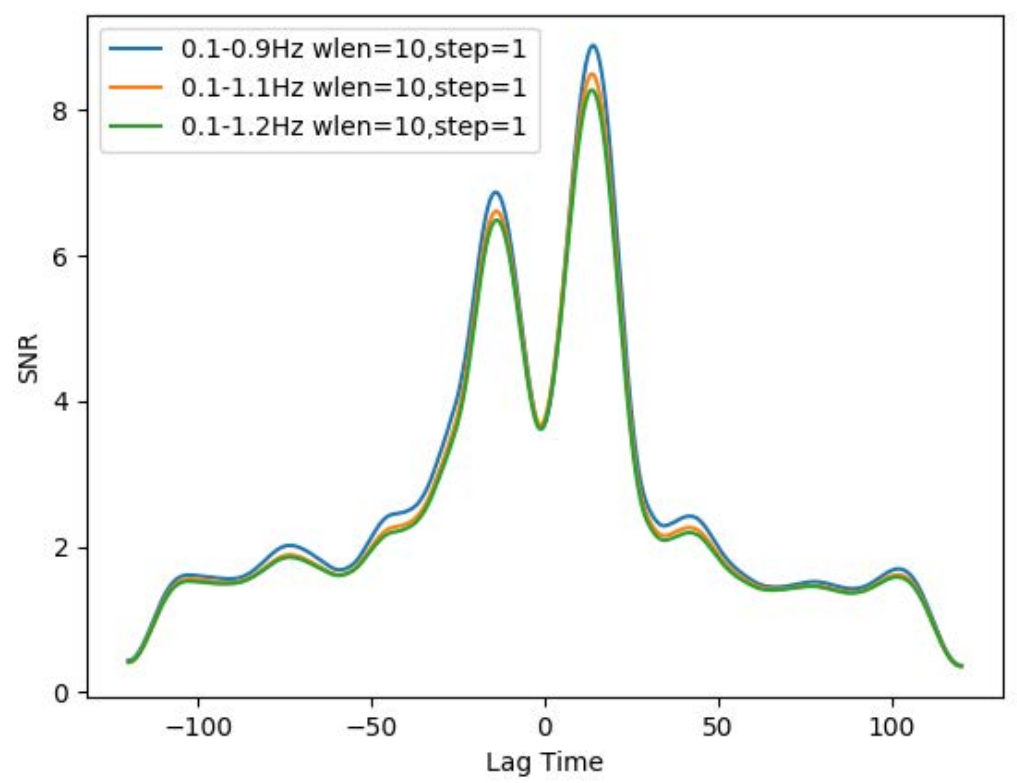

Figure 4.11: SNR filters test for the two-year dataset from the $1^{\text {st }}$ of January 2016 to the $28^{\text {th }}$ of February 2018 for IKR-MLF using a 30-day stack size. This shows that as the frequency range increases, the SNR values get slightly worse. Changing the MWCS window length and the MWCS step does not affect the SNR but does change the coherency (see Figure 4.12).

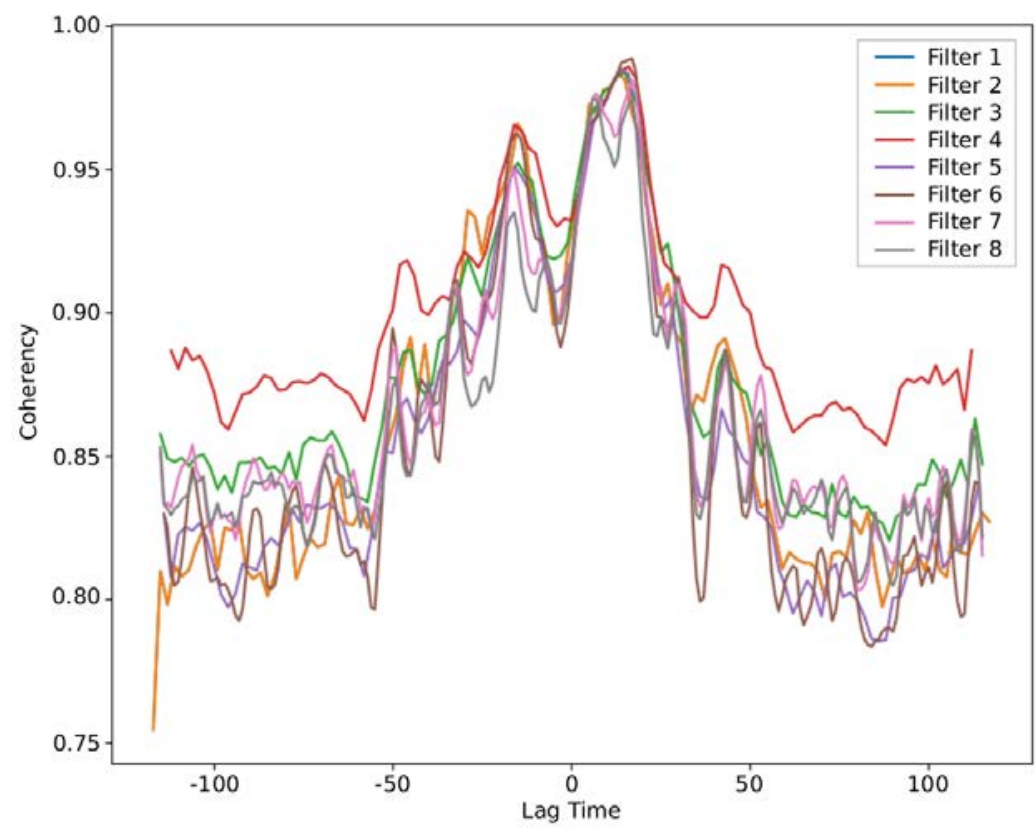

Figure 4.12: Coherency filters test for the two-year dataset from the $1^{\text {st }}$ of January 2016 to the $28^{\text {th }}$ of February 2018 for IKR-MLF using a 30-day stack size. Filters listed are those shown in Table 4.1. The filter with the highest coherency is $0.1-0.9 \mathrm{~Hz}$ with a window length of $16 \mathrm{~s}$ and a step of $2 \mathrm{~s}$. As this is our highest tested value, greater values for the window length and step are tested in Section 4.2.7. 
This filter (filter number 4 in Table 4.1) produced a high coherence value (shown in Figure 4.12) for the IKR-MLF pair for the ZZ component. This resulted in it being possible to raise the minimum coherence parameter value from the default value of 0.65 to a value of 0.9 . In Figure 4.13, SNR and coherency values are plotted against one another for the different filters shown in Table 4.1. This figure shows a direct relationship as when one increases, so does the other. From this figure it is also possible to see that if the minimum coherence is set to 0.9 then the lowest SNR values would be removed $(\mathrm{SNR}<2)$.

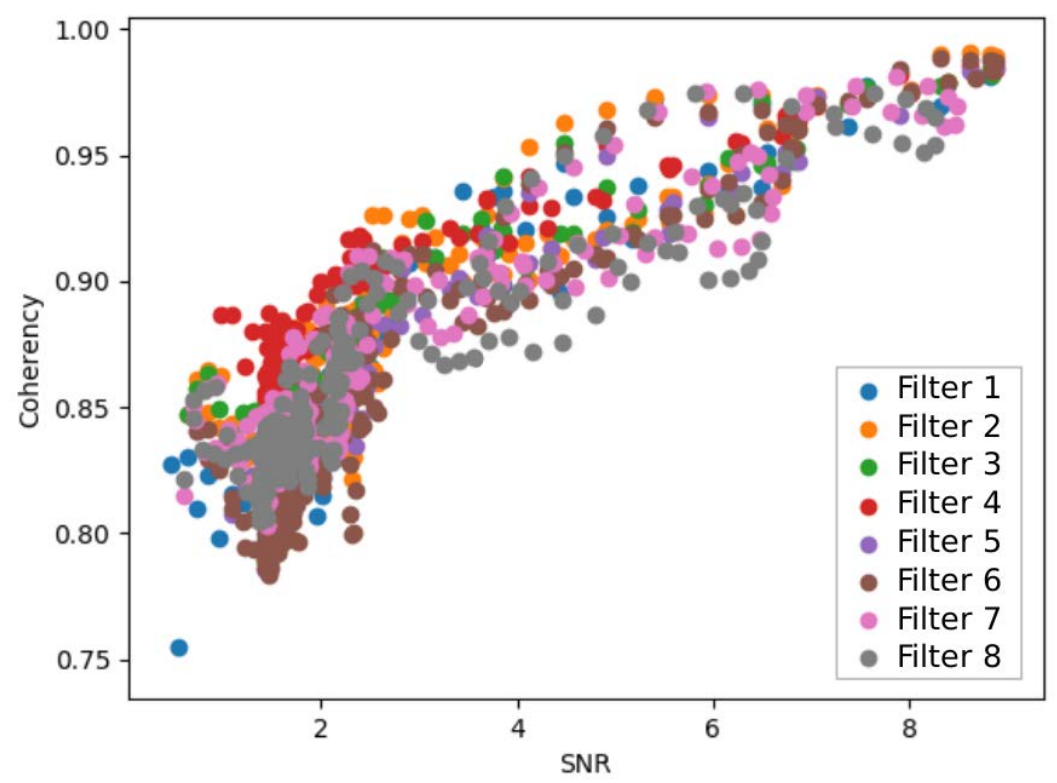

Figure 4.13: SNR and coherency filters test for the two-year dataset. This is plotted from the results of Figures 4.11 and 4.12 against one another. This plot shows that using a filter with a frequency range from $0.1-0.9 \mathrm{~Hz}$, a window length of $16 \mathrm{~s}$ and a step of $2 \mathrm{~s}$ shows the best overall result. Filter 2 in Table 4.1 also produces a good result but does not produce very high results for the coherency (Figure 4.12).

From these tests we determined that using a whiten filter of 0.1-0.9 Hz, a MWCS filter of $0.15-0.85 \mathrm{~Hz}$, MWCS window length of $16 \mathrm{~s}$ and a MWCS step of $2 \mathrm{~s}$, gave the best result. However as a few other filters also produced decent results, they were kept in when processing the cross-components for two-years worth of data. The filters shown in those tests are shown in Table 4.3.

The length of the cross-correlation time slices was also another important decision. The cross-correlation time slice is the length of data that is used in the signal window which then for each filter, has the traces 1-bit normalized and whitened in 
the frequency domain. In earlier tests it was shown that a value of $1800 \mathrm{~s}$ for the cross-correlation time slice would be sufficient (shown in Figure 4.7). However, upon further reading it was determined that a lower cross-correlation time slice value could also be an option as it had been used before for short period stations looking at low frequency noise (Civilini et al., 2020). Civilini et al. (2020) used a value of $900 \mathrm{~s}$ for their analysis of ambient noise on a geothermal field using short period instruments. As the lowest value that we had currently used was $1800 \mathrm{~s}$, it was deemed worthwhile to test lower values until no improvement was achieved. Also as another study had also seen benefits in using a value smaller than 1800 s (Civilini et al., 2020), both $900 \mathrm{~s}$ and a smaller value of $450 \mathrm{~s}$ was tested. This figure (Figure 4.14) shows that a value of $900 \mathrm{~s}$ gives the best result because it produces the highest overall SNR values over the entire lag time. A smaller value of 450 s produced the same result as $900 \mathrm{~s}$ did and would increase the computation time. So, $900 \mathrm{~s}$ was chosen for the cross-correlation time slice value.

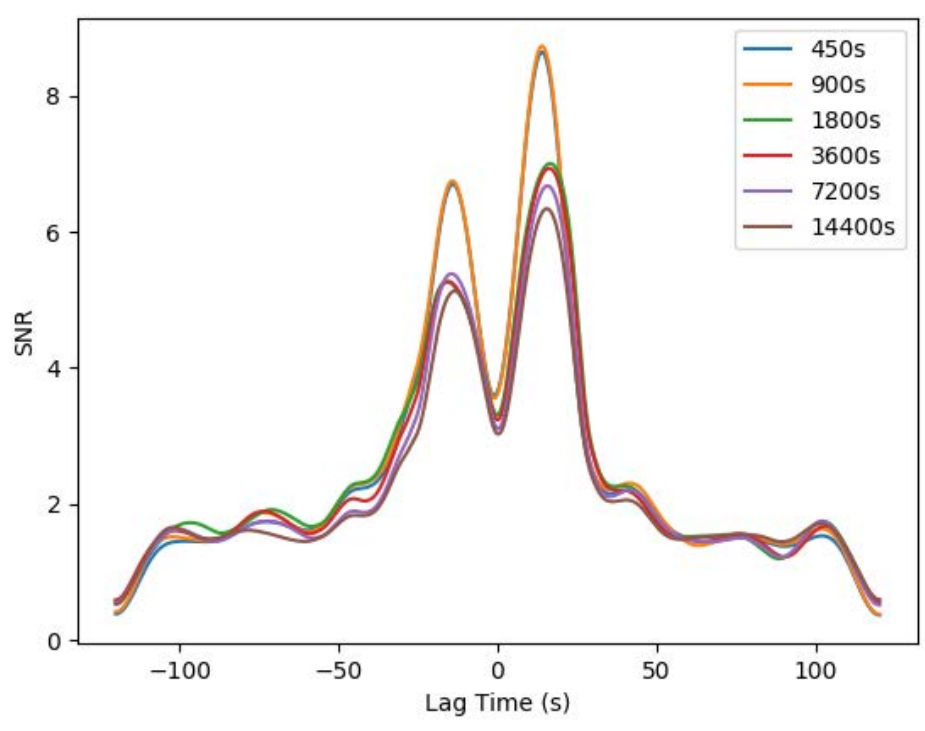

Figure 4.14: SNR plot showing different cross-correlation time slice values. $900 \mathrm{~s}$ was chosen as the best value because $450 \mathrm{~s}$ does not increase the SNR value but would increase the computation time.

Coherent energy was also checked with the two-year full station dataset. The following figures, Figures 4.15 and 4.16, show the daily cross-correlation stacks for 10-day stacks against lag time for the ZZ component using filter 2 in Table 4.1. These 
Figures, 4.15 and 4.16, show the coherent energy arrivals for station pair IKR-MLF and SJQ-WJM respectively.
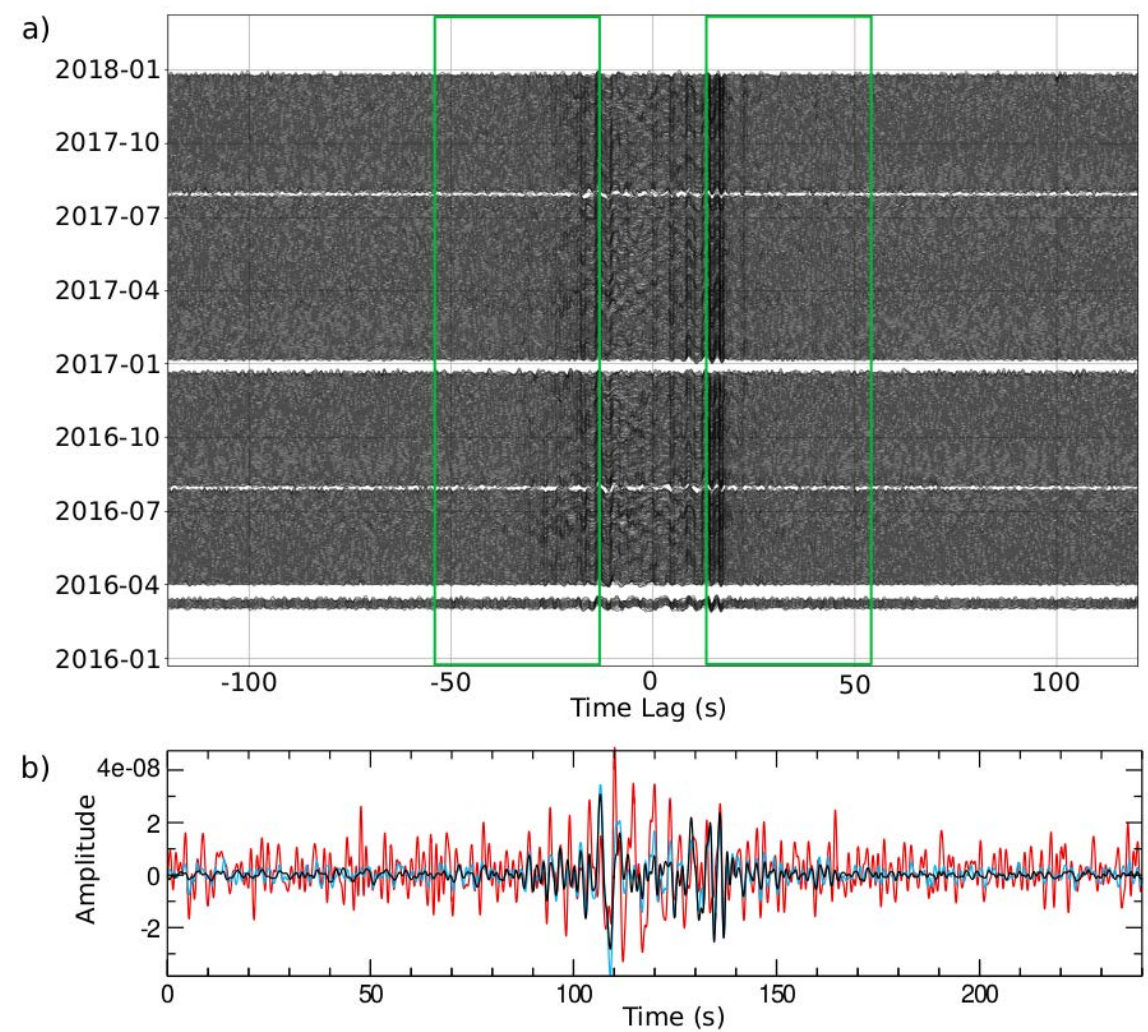

Figure 4.15: Cross-correlation stability and quality for station pair IKR-MLF. a) Amplitude for 10-day stacks over the reference time frame (January 2016 to July 2016). Green boxes indicate DTT windows. The lack of traces at a few time periods are due to IKR and MLF missing data (see Figure 3.2). b) Waveform of crosscorrelations, red - daily cross-correlations, blue - 10-day stack, black - reference stack January 2016 to July 2016.

When a stack begins, it takes the first daily stack and then adds on the remaining available days before it moves onto the next moving stack. For example, for a 10-day moving stack, on the 11th available day, the 10-day moving stack will contain data from days 2-11, for the 12 th day, data from days $3-12$. This has an effect on days where there is no data as the moving stack on the first day with no available data will contain all of the previous days before it. This is seen in the coherent energy plots (Figures 4.15 and 4.16) for the 10-day stack (and 1-day stack in Figure 4.16), as the width of the missing data gaps are slightly shorter than those in the missing data availability plot (Figure 3.2). In these Figures, 4.15 and 4.16, the amplitude of different stacks are also shown. At the bottom of each figure, an amplitude plot 

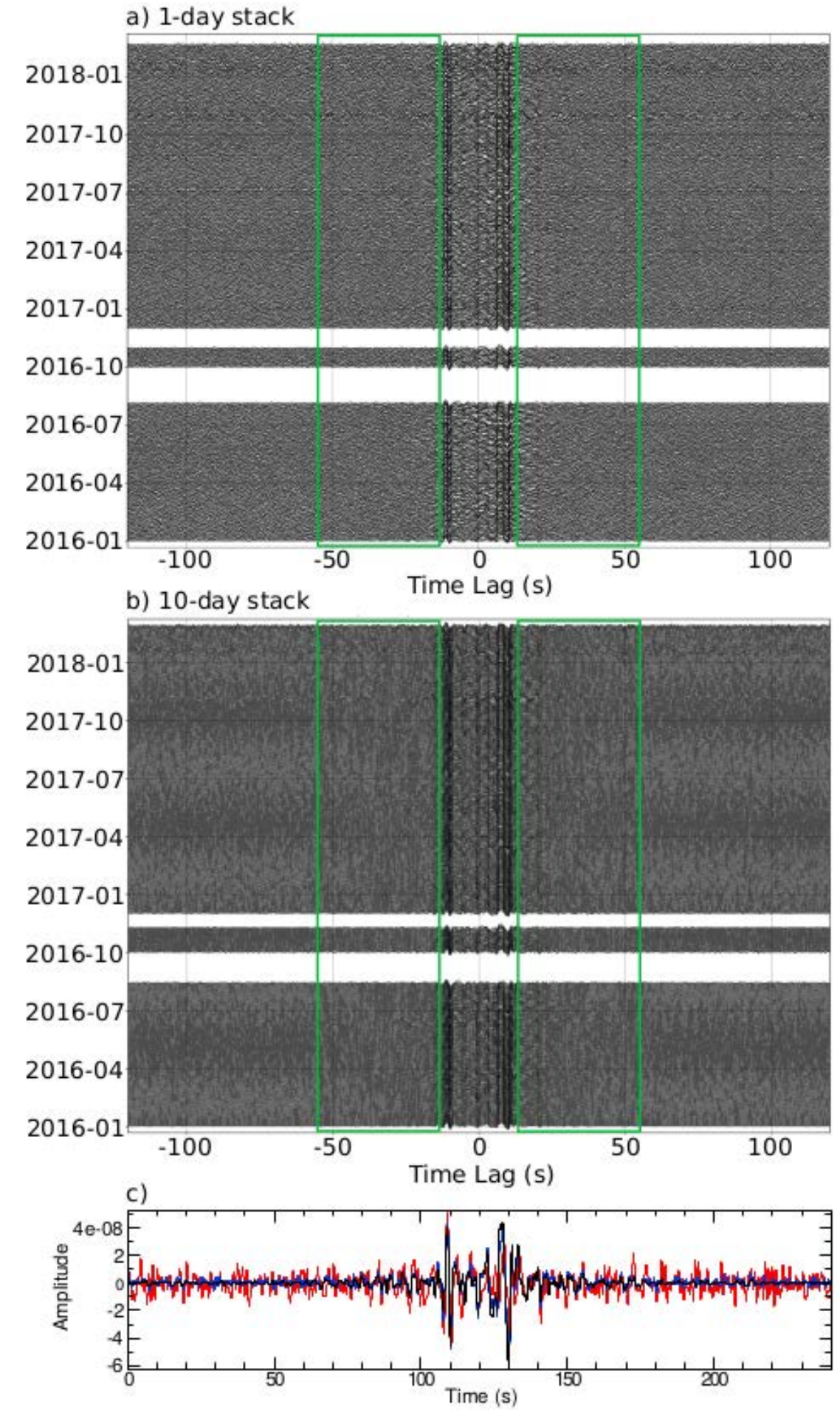

Figure 4.16: Cross-correlation stability and quality for station pair SJQ-WJM. a) Amplitude for 1-day stacks over the reference time frame (January 2016 to July 2016). b) Amplitude for 10-day stacks over the reference time frame (January 2016 to July 2016). Green boxes indicate DTT windows. Both SJQ and WJM are missing data from August 2016 - October 2016 and SJQ again in December 2016 shown in Figure 3.2. c) Waveform of cross-correlations, red - daily cross-correlations, blue 10-day stack, black - reference stack January 2016 to July 2016. 
of the daily stacks (in red), the 10-days stacks (in blue) and the reference stack (in black) is shown. This can be used to determine if all of the different stacks are matching well against one another. For these two station pairs and for this filter, Filter 2 in Table 4.1, it appears that they match well. This filter had quite a low MWCS window length $(10 \mathrm{~s})$ and MWCS step (1s). As this filter showed promising results, it was used to calculate DVV plots. Upon using this filter, the velocity variation plots were more erratic whereas when a larger MWCS window length and MWCS step were used (Filter 4 in Table 4.1) the results were smoother.

The DTT windows can also be determined from these coherent energy plots. From Figures 4.15 and 4.16 it was determined that the minimum lag would be $15 \mathrm{~s}$ and the width of the time lag window would be 40s (shown in green in these figures). The DTT windows would go from $-55 \mathrm{~s}$ to $-15 \mathrm{~s}$, and $+15 \mathrm{~s}$ to $55 \mathrm{~s}$, as we are using both sides of the data. These windows were chosen as it would contain data directly after the direct waves have arrived and also contain the important section of the coda before the amplitude became too small. Also at this stage the decision was made to use a static shift instead of a dynamic shift. When a dynamic shift is chosen the inter-station distance is used to determine the minimum time lag. This lag is calculated from the distance and the velocity provided. This velocity is determined by the user so that the minimum lag does not include ballistic waves (Lecocq et al., 2014). Examples of both static and dynamic lag are shown in Figure 4.17.

Static shift was chosen as the interstation distance between most of the station pairs that can be used is quite small $(\sim 80 \mathrm{~km})$. Therefore we would not need to worry about changing the velocity to determine the lag time from the distance as they would all have a similar velocity and would not change much.

Another important decision was using forced DVV plots instead of unforced. Forced DVV plots are when the weighted linear regression is forced to pass through the origin $(0,0)$, and unforced is when there is a constant added to allow for the weighted linear regression to be offset from the origin. The difference between a forced linear regression and an unforced linear regression is quite noticeable. This is shown in the 


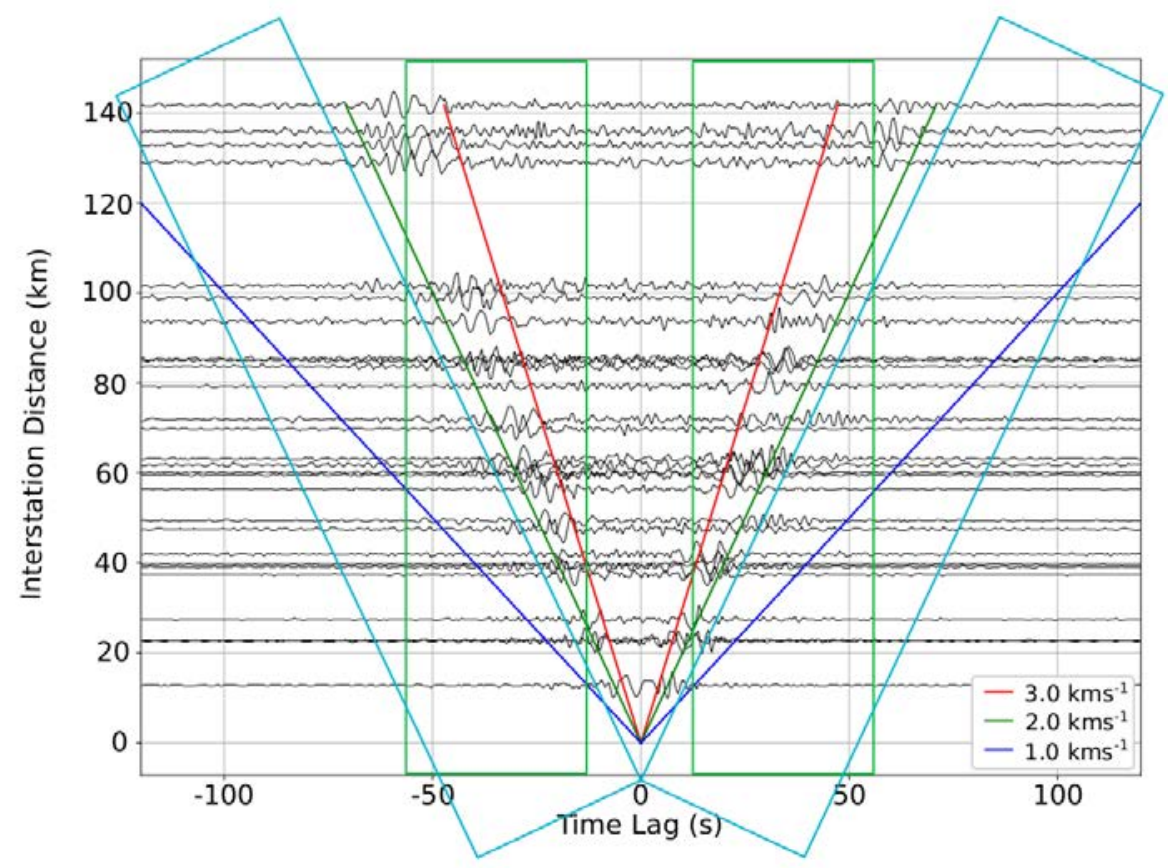

Figure 4.17: Distance plot of static and dynamic shift. A static shift (in green) is calculated independent of velocity whereas a dynamic shift (in blue) is calculated using a velocity specific to the region of interest.

following Figure 4.18.
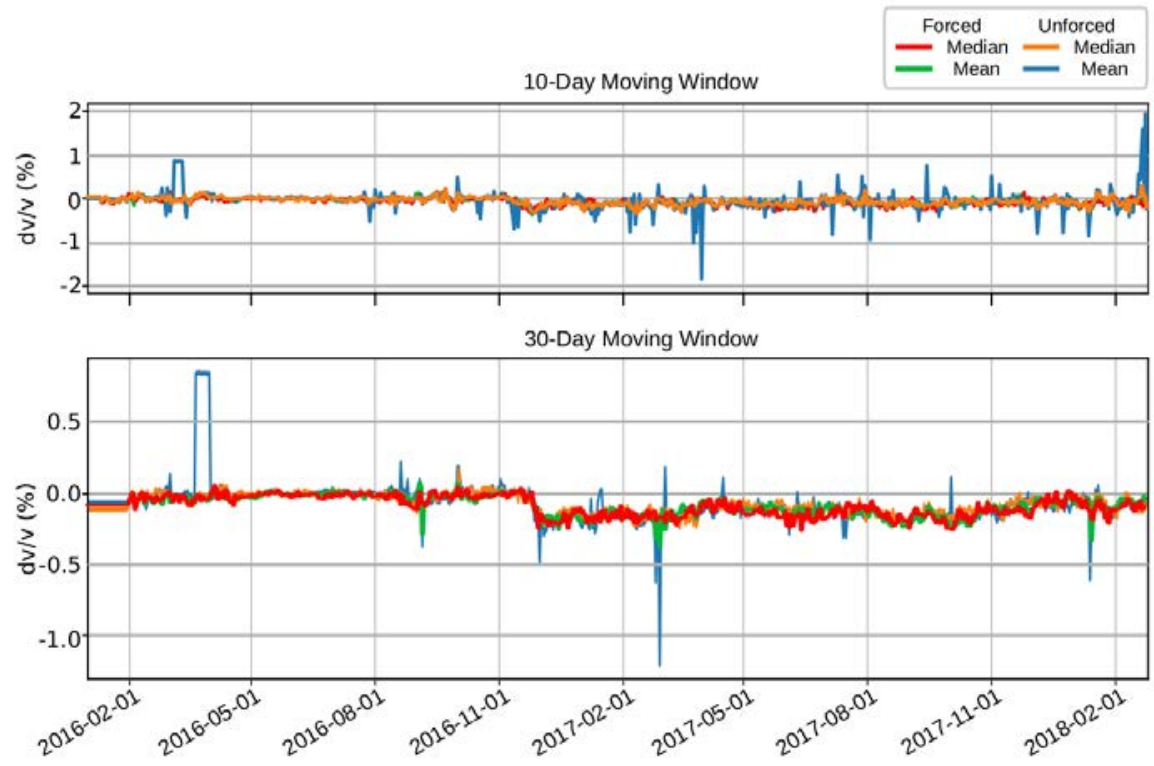

Figure 4.18: DVV plot showing forced and unforced linear regression for the verticalvertical component using Filter 2 in Table 4.1 for a 10-day and a 30-day moving window. In the 10-day moving window the unforced results are in the foreground and in the 30-day moving window the forced results are in the foreground. The velocity changes are calculated for each station pair and then the median and mean values are computed within MSNoise.

The reason for allowing the weighted linear regression to cross the axis elsewhere than 
on $(0,0)$ is, for example, to study the potential clock drifts or noise source position variations (Lecocq et al., 2014). As this should not be a problem with our dataset we will use forced linear regression (crossing the axis at $(0,0)$ ). It also shows a marked improvement on the result as shown in Figure 4.18. In the unforced moving windows (Figure 4.18) the mean is much higher and skews the median values. This results in very high velocity variation values such as $\pm 1 \%$, which is quite high compared to values shown in the forced moving windows (Figure 4.18) where the median matches the mean well and there is a $\pm 0.25 \%$ change in the velocity variation. Both the median and the mean are calculated from all of the station pairs within MSNoise. The median velocity value is the middle value of all of the station pairs for that day and the mean is calculated from all of the station pairs and divided by the number of pairs used for a particular day. As the mean can be skewed by a strong outlier from a single station pair, the median was the preferred choice for the velocity variation plots.

Importantly at this stage, we see a decrease of $\sim 0.2 \%$ change in velocity in the forced DVV plot (Figure 4.18) following the 2016 Kaikōura earthquake. Previous studies have also observed a seismic velocity decrease following large earthquakes (see Background Theory chapter). The next step was to test this for all of the cross-components over a two-year period and then trial it for the full period of seven years.

Once the parameters had been determined for the ZZ component, the reference stack was changed from the $1^{\text {st }}$ of January 2016 to $30^{\text {th }}$ September 2016, to a period from $1^{\text {st }}$ January 2016 to the $1^{\text {st }}$ of July 2016 . This made the period slightly smaller (six months instead of nine months) but should still be able to accurately portray the background noise. For the cross-component tests, the latter reference period is used. When testing for the full seven-year period, a reference period of seven years was also trialed to see how it affects the results; see Sections 5.2.2 and 5.3.2. 


\subsubsection{Cross-Component tests for two years}

In testing the different cross-components, the first step was determining which stations for both cross-pairs produced good results by themselves. For this, we analysed the data availability table (Figure 3.2) and decided to test the stations shown in Table 4.2 .

\begin{tabular}{c|c} 
Component & Stations \\
\hline ZZ & IKR, JSP, MLF, SJQ, SVR, WJM. \\
EE & CVR, IKR, JSP, KVR, MHR, MLF, MTV, SJQ, WJM. \\
NN & CCB, JSP, MHR, MLF, SJQ, SVR, WJM. \\
EZ/ZE & IKR, JSP, MLF, SJQ, WJM. \\
EN/NE & JSP, MHR, MLF, SJQ, WJM. \\
NZ/ZN & JSP, MLF, SJQ, SVR, WJM.
\end{tabular}

Table 4.2: Stations for different cross-components for the two-year dataset.

Stations shown in Table 4.2 have at least $75 \%$ of the data for a two-year period. These stations also had to have at least six months of data both before and after the 2016 Kaikōura earthquake. All of these different station pairs and cross-components were tested using the filters shown in Table 4.3. The filter that produced the best result for the ZZ component was filter number 4 in Table 4.1 (this is the same filter as filter number 1 in Table 4.3). As this filter was successful and filters with lower MWCS window length and step were not successful, higher window length and step values will now be tested using two frequency ranges $(0.1-0.9 \mathrm{~Hz}$ and $0.1-1.1 \mathrm{~Hz})$ for all cross-components.

\begin{tabular}{l|l|l|l|l} 
Filter number & Whiten Filter & MWCS Filter & $\begin{array}{l}\text { MWCS Win- } \\
\text { dow Length }\end{array}$ & MWCS Step \\
\hline 1 & $0.1-0.9 \mathrm{~Hz}$ & $0.15-0.85 \mathrm{~Hz}$ & $16 \mathrm{~s}$ & $2 \mathrm{~s}$ \\
2 & $0.1-0.9 \mathrm{~Hz}$ & $0.15-0.85 \mathrm{~Hz}$ & $16 \mathrm{~s}$ & $4 \mathrm{~s}$ \\
3 & $0.1-0.9 \mathrm{~Hz}$ & $0.15-0.85 \mathrm{~Hz}$ & $20 \mathrm{~s}$ & $4 \mathrm{~s}$ \\
4 & $0.1-1.1 \mathrm{~Hz}$ & $0.15-1.05 \mathrm{~Hz}$ & $16 \mathrm{~s}$ & $2 \mathrm{~s}$ \\
5 & $0.1-1.1 \mathrm{~Hz}$ & $0.15-1.05 \mathrm{~Hz}$ & $16 \mathrm{~s}$ & $4 \mathrm{~s}$
\end{tabular}

Table 4.3: Table of filters used to test all cross-component pairs for the two-year dataset.

In using these results the following velocity variation plots were produced (Figures 4.19 - 4.26) for each filter listed in Table 4.3 for each cross-component. The possibility that 
a few station pairs should be removed was checked by using correlation coefficiency plots for each cross-component.

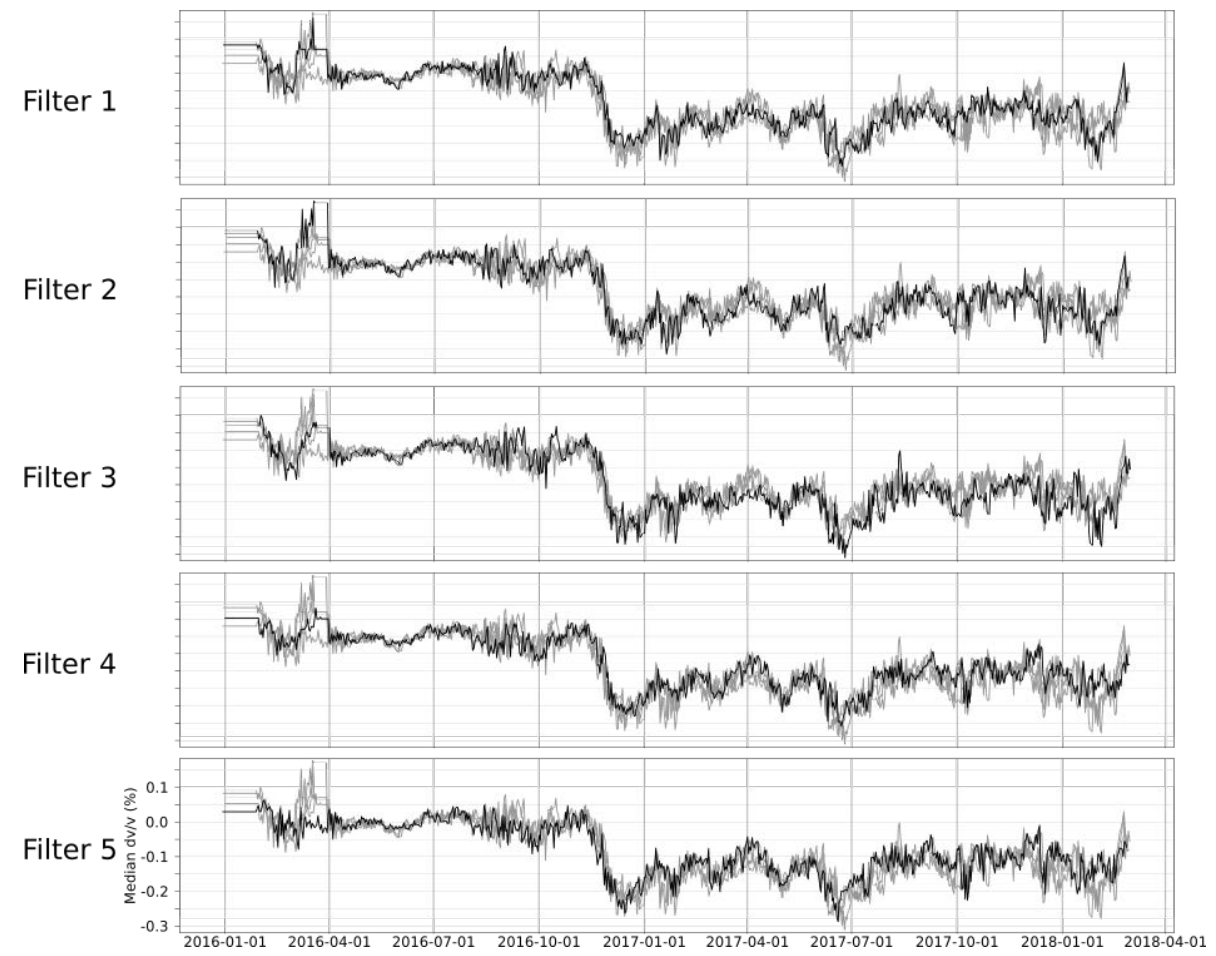

Figure 4.19: DVV plot for the East-East component for five different filters in Table 4.3. Axes values for Filters 1-4 are the same as 5 .

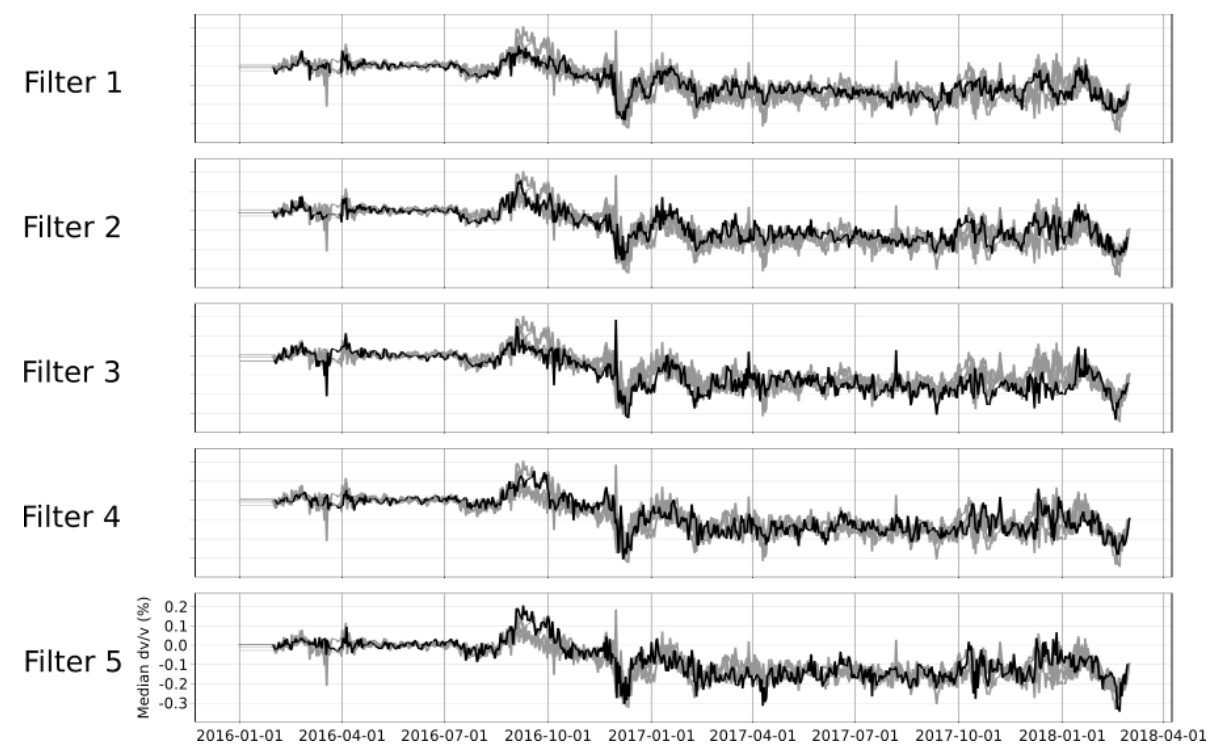

Figure 4.20: DVV plot for the North-North component for five different filters in Table 4.3. Axes values for Filters 1-4 are the same as 5 . 


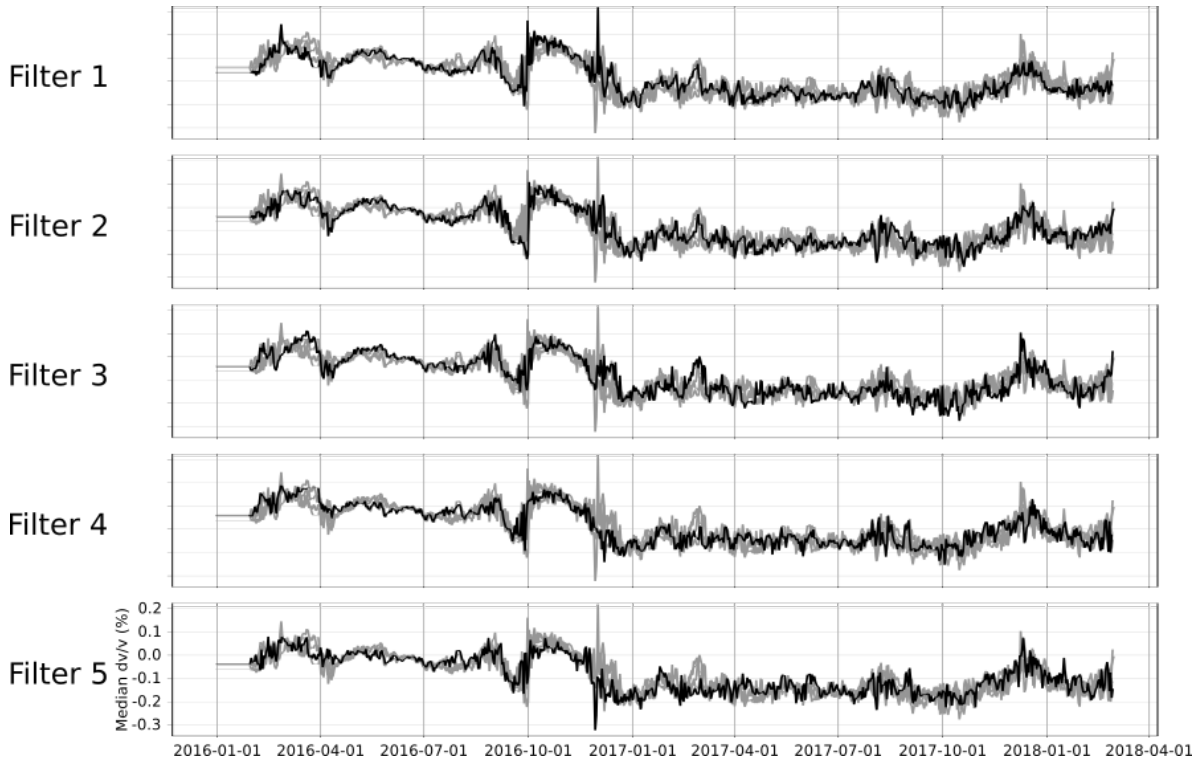

Figure 4.21: DVV plot for the East-North component for five different filters in Table 4.3. Axes values for Filters 1-4 are the same as 5 .

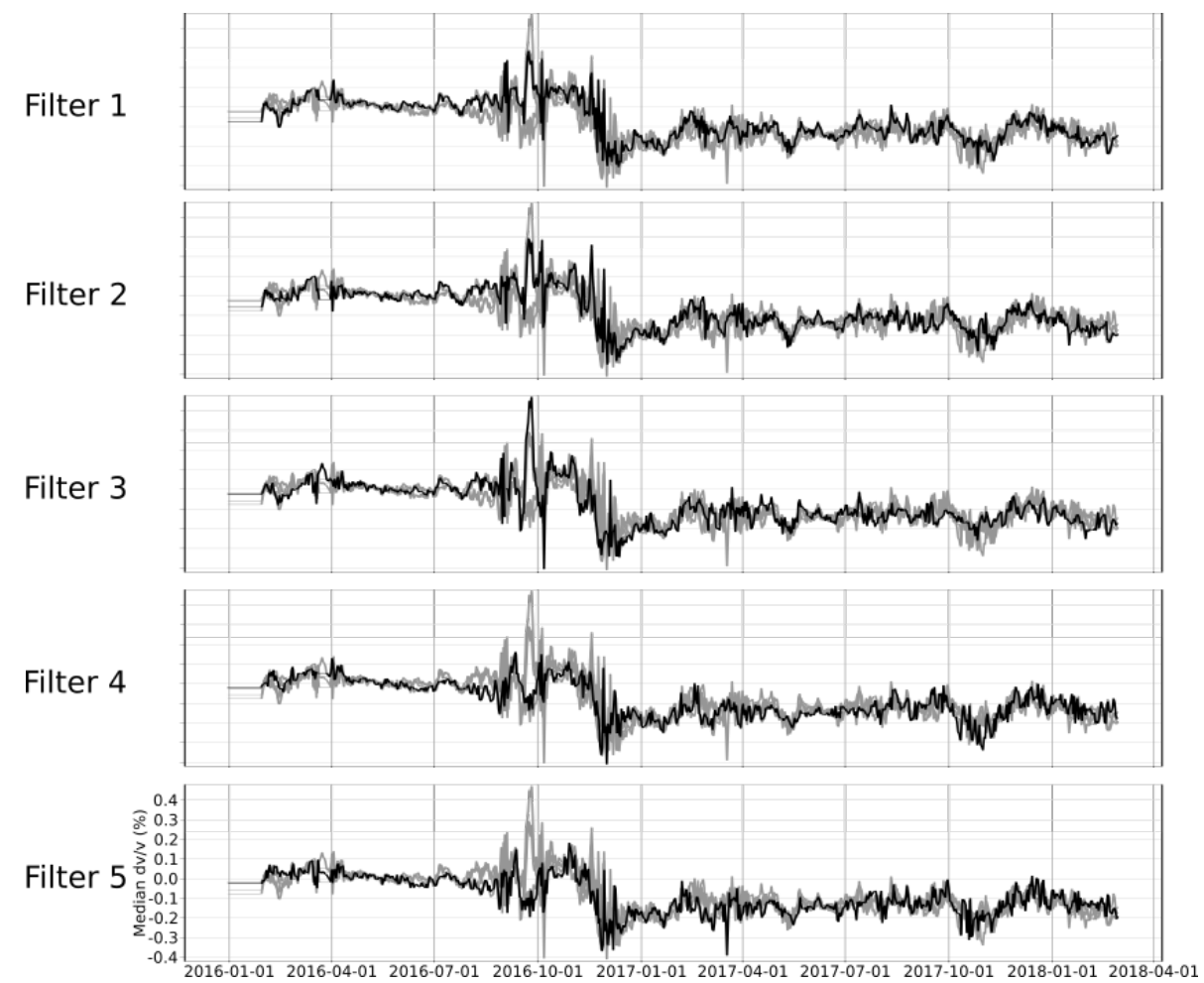

Figure 4.22: DVV plot for the North-East component for five different filters in Table 4.3. Axes values for Filters 1-4 are the same as 5 . 


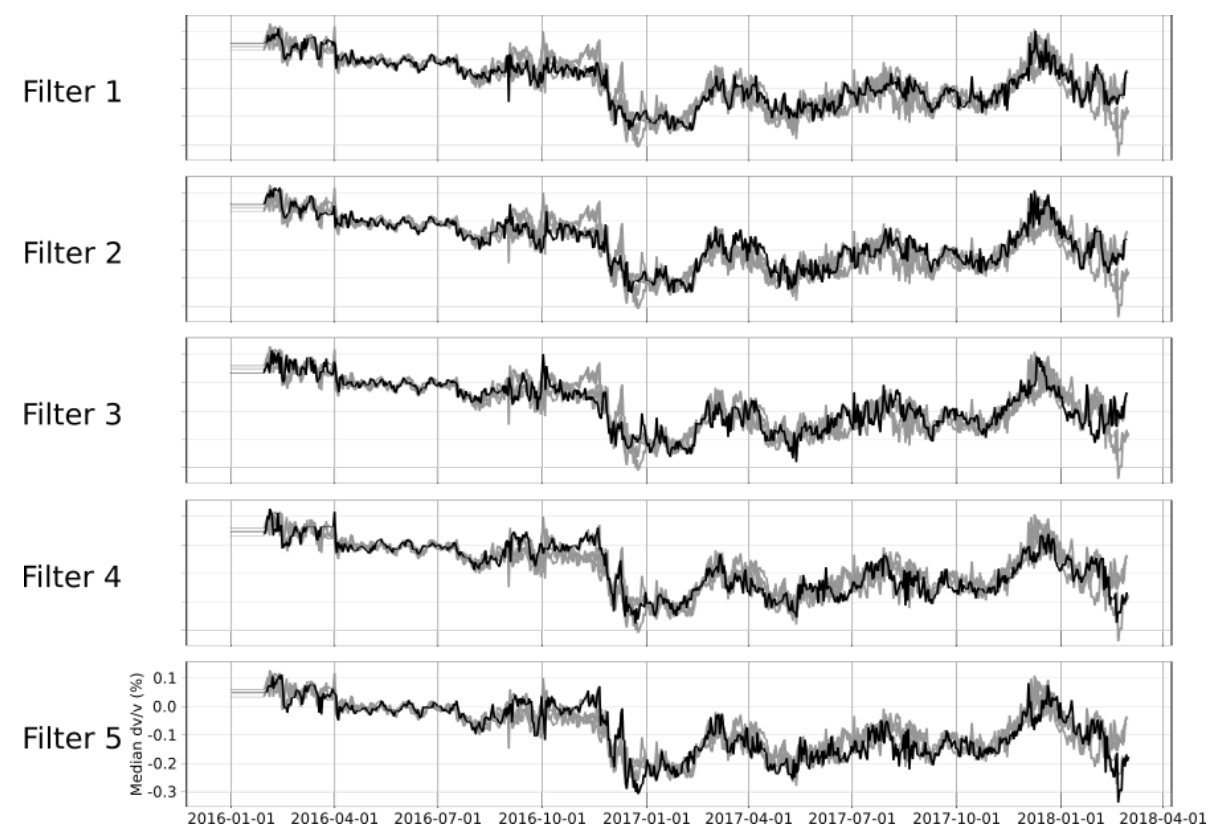

Figure 4.23: DVV plot for the East-Vertical component for five different filters in Table 4.3. Axes values for Filters 1-4 are the same as 5 .

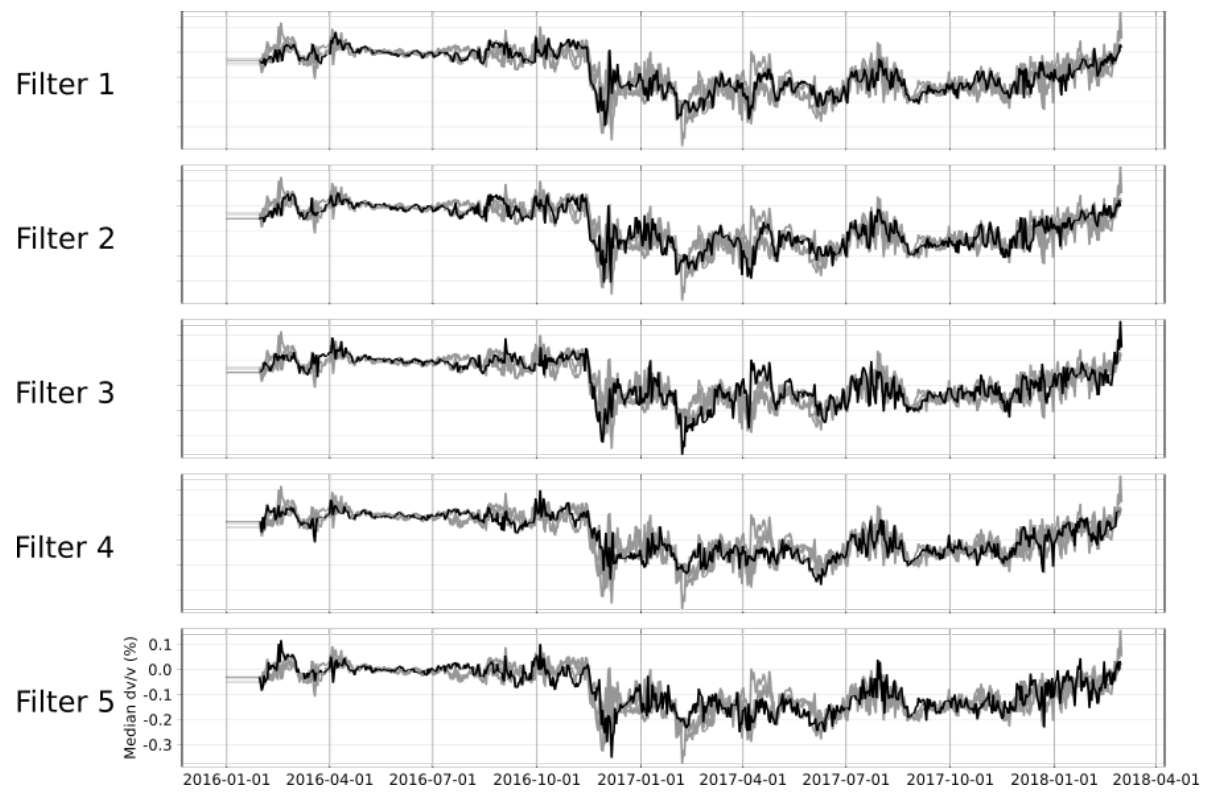

Figure 4.24: DVV plot for the Vertical-East component for five different filters in Table 4.3. Axes values for Filters 1-4 are the same as 5 . 


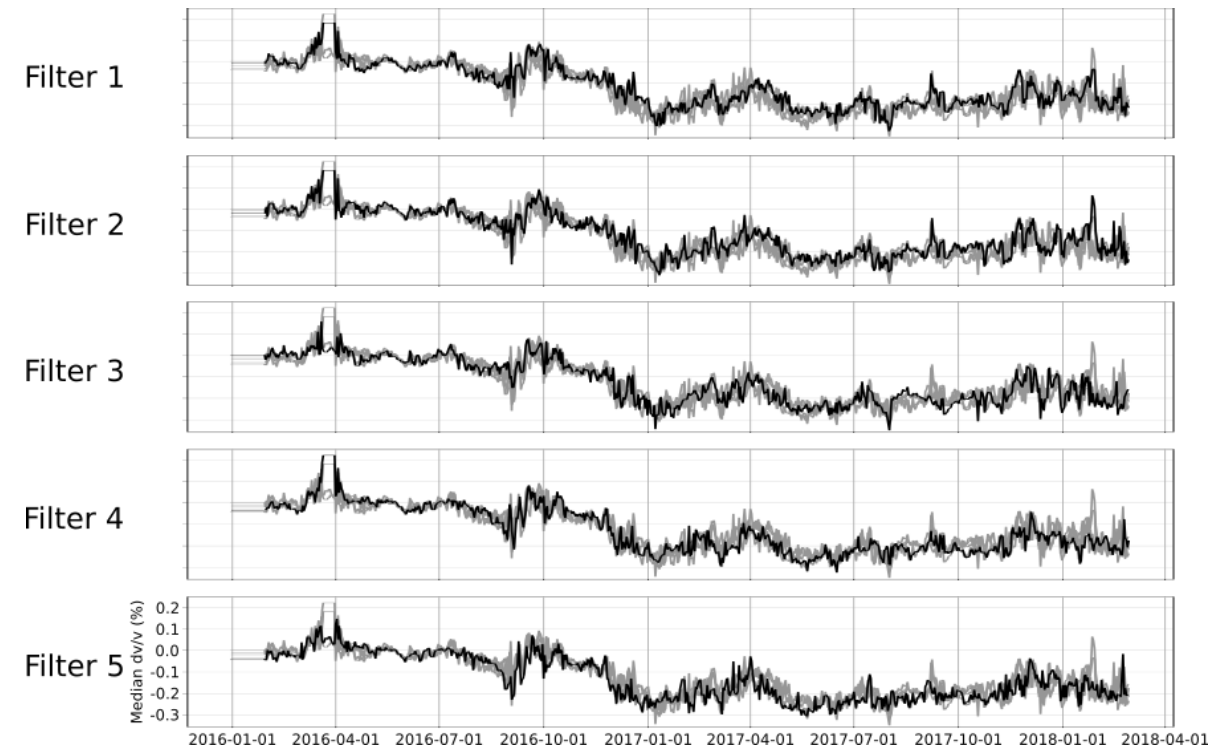

Figure 4.25: DVV plot for the North-Vertical component for five different filters in Table 4.3. Axes values for Filters 1-4 are the same as 5.

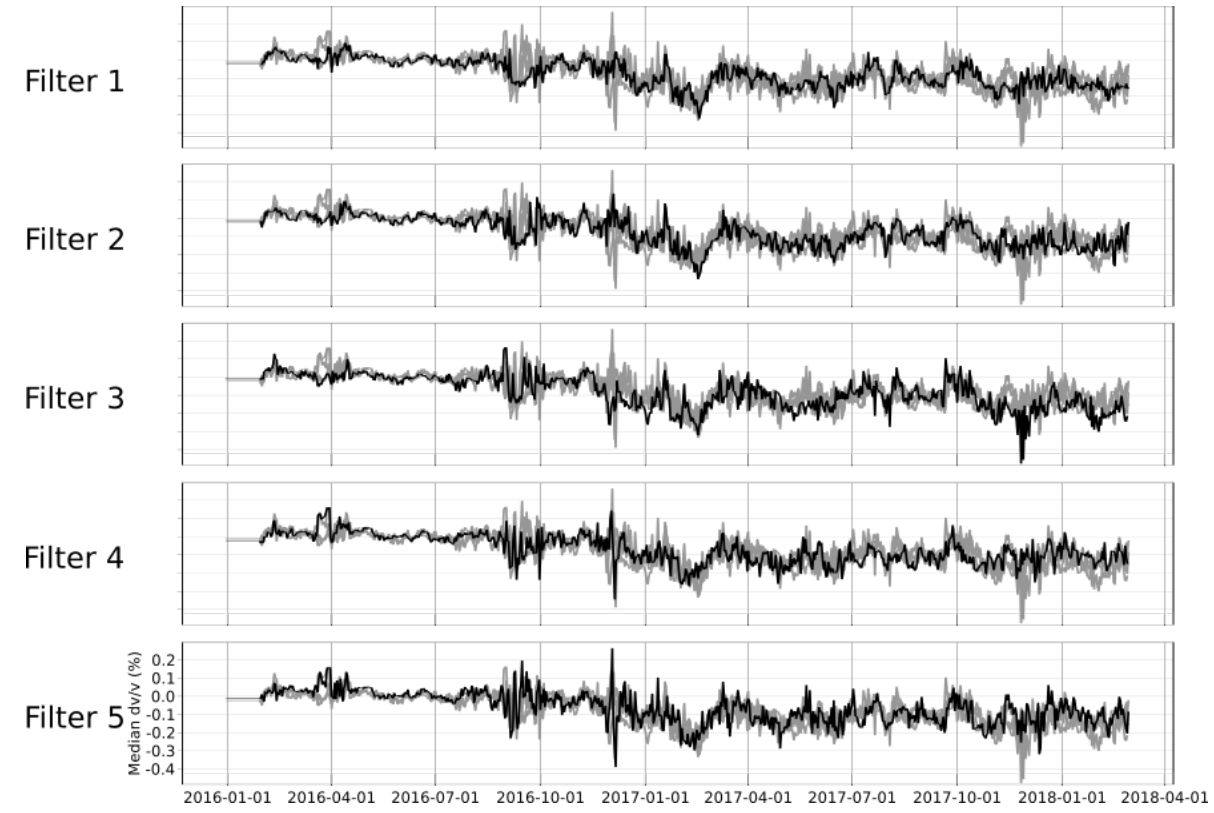

Figure 4.26: DVV plot for the Vertical-North component for five different filters in Table 4.3. Axes values for Filters 1-4 are the same as 5 . 
In all of these Figures (4.19-4.26) a velocity decrease was visible after the 2016 Kaikōura earthquake. Different cross-component results are discussed in the Results chapter for a two-year and a seven-year dataset.

\subsubsection{Full Dataset - seven years, 12 stations}

Finally all current parameters were applied to the full dataset for the ZZ component. This test was performing using the same parameters chosen for the two-year dataset with the same filters shown in Table 4.3. The following stations were included in this test: CCB, CVR, IKR, JSP, MLF, SJQ, SRB, SVR, and WJM. This involved trying stations with three or more years of data from a period from $1^{\text {st }}$ of January 2012 to the $28^{\text {th }}$ of February 2018. All stations had to have at least one year of data before the 2016 Kaikōura earthquake and six months of data after the earthquake to be used in this analysis. This produced the following result (Figure 4.27) using a two-and-a-half-year reference period for a 30-day moving window. Correlation coefficient plots determined that only CCB produced poor results, so this station is removed from any further more tests of the $\mathrm{ZZ}$ component. We note there is evidence to suggest this is potentially the station in CCB-CVR pair that produced the poor CC result in Figure 4.8.

The full seven-year dataset was also used to test whether a reference period of the whole testing period would be desirable. This found promising results and all different reference periods, including that shown in Figure 4.27, are discussed more fully in the Results chapter. By using larger reference periods, the velocity change values before the 2016 Kaikōura earthquake are also slightly increased, as using a reference period that includes the earthquake skews the velocity changes by increasing the values before the earthquake. Further analysis of different reference periods are compared in the Results chapter. The results for all of the cross-component pairs are all shown in the Results chapter for a two-year and a seven-year dataset. 


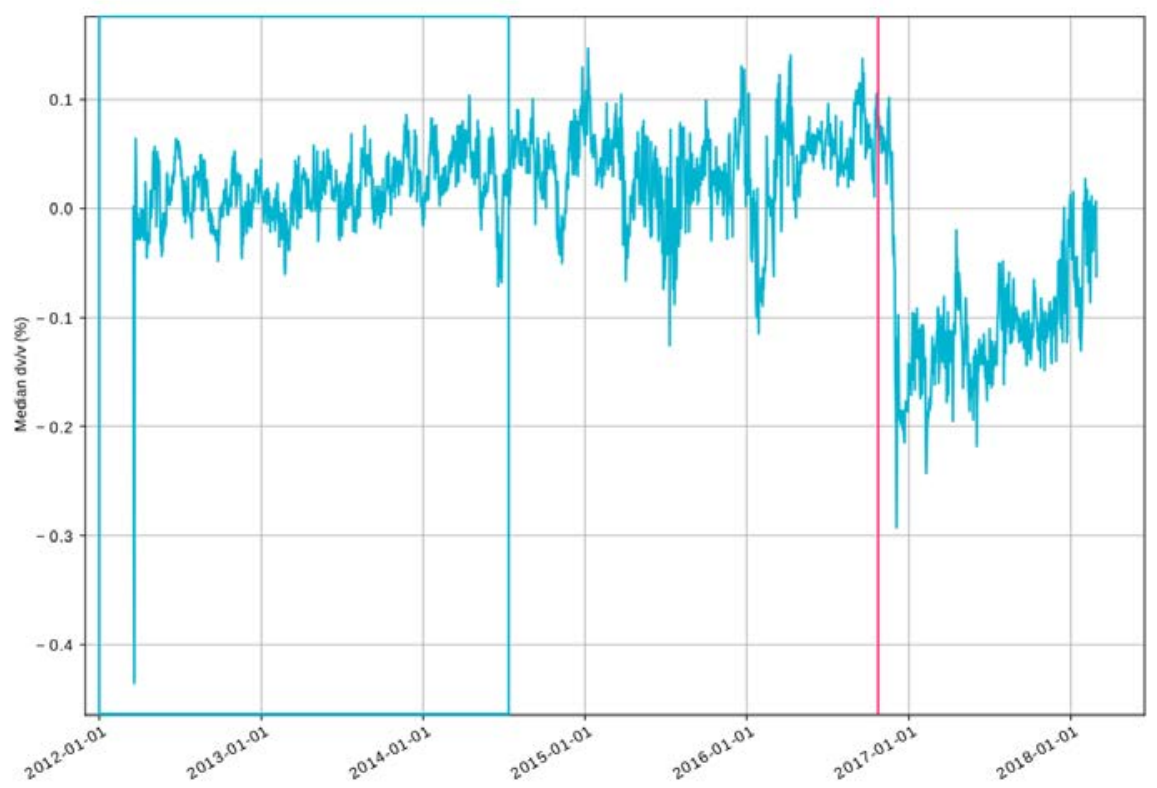

Figure 4.27: Velocity changes using a two-and-a-half-year reference period for the vertical-vertical component pairs for a 30-day moving windows for seven years of data. 


\section{Chapter 5}

\section{Results}

This chapter presents the velocity change results recovered after processing crosscomponent ambient noise DPRI data. Firstly the stability of the velocity changes for different cross-component pairs are presented (Section 5.1). Secondly, we describe the results of the two-year test period for the different cross-component pairs (Section 5.2). Upon receiving convincing results for the two-year period, the data period was lengthened to seven years. The results for the different cross-components are shown in Section 5.3. For both Sections 5.2 and 5.3, different stations will either be excluded or included depending on quality and duration, and this will be discussed in both sections.

\subsection{Stability of Velocity Changes}

In this section multiple cross-component pairs will be compared to determine if they are all producing stable velocity change results. This section and Section 5.2 provides the results for different cross-components for a dataset slightly greater than two years (from the $1^{\text {st }}$ of January 2016 to the $28^{\text {th }}$ of February 2018). The same parameters that were applied to the vertical-vertical (ZZ) component that was discussed in Section 4.2 were also applied to all of the component pairs. The only difference in the cross-component pairs is that different stations were used for each individual 
component pair. This is a result of each station having different data available for individual components at each seismometer site (shown in the data availability plot - Figure 3.2). As not enough stations had East and North components operating at the same time, radial and transverse components were not computed. To produce a usable result, each station's components must provide data with a high correlation coefficient value of at least $70 \%$ for one-and-a-half years of the two-year dataset. These stations were all determined in Section 4.2 and will be shown again in Section 5.2.1.

Another important consideration is coherent energy. If there is a real signal, this will be observed in the coherent energy recorded travelling across all component pairs from different seismic stations. As the interstation distance increases, we would expect to see direct arrivals arriving at ever increasing lag times. Figure 5.1 demonstrates this by showing reference cross-correlation functions against interstation distance for all nine cross-component pairs for the seven-year dataset. The seven-year dataset will be more closely examined in Section 5.3. Clear arrivals are observed in the cross-component functions that use component pairs with either the East or the Vertical component. The clearest arrivals are observed in the cross-component functions where the same component is used at both stations (ZZ and EE). We also conclude that the best result that includes the North component is the pair that uses both the north component at each station $(\mathrm{NN})$. Using radial, transverse, and vertical cross-components would make it clearer to be able to compare these results to what we would expect from surface waves; Rayleigh and Love Waves.

To compute radial and transverse waves, we would need stations to have both north and east components for the same duration. For the two-year dataset only four stations have this: JSP, MLF, SJQ and WJM and for the seven-year dataset only two stations could be used: SJQ and WJM. As a result of this, radial and transverse waves were not computed by rotating the east and north components to give Rayleigh wave energy or Love wave energy respectively. Rayleigh wave energy is also visible on the vertical component so Rayleigh energy is visible on the $\mathrm{ZZ}$ pair shown in Figure 

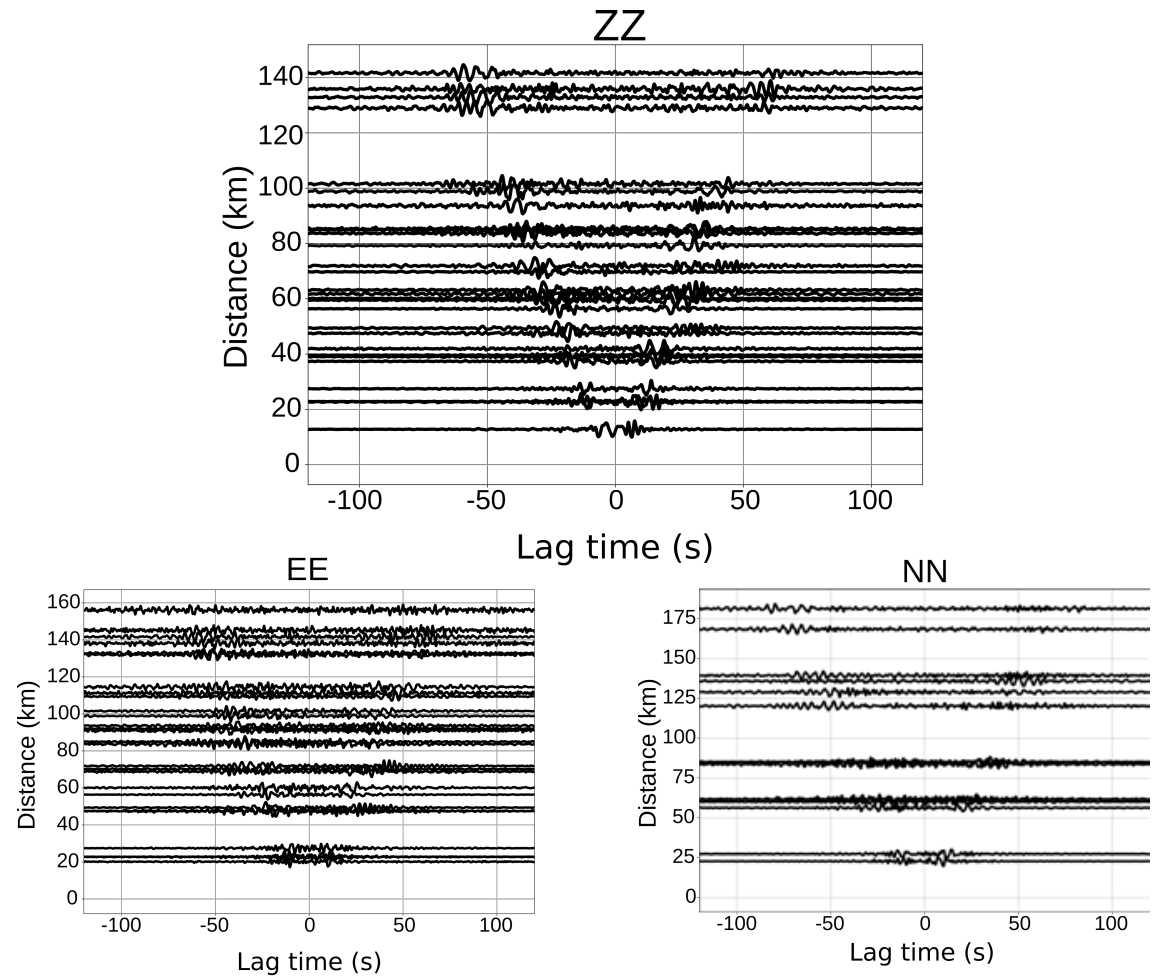

EZ

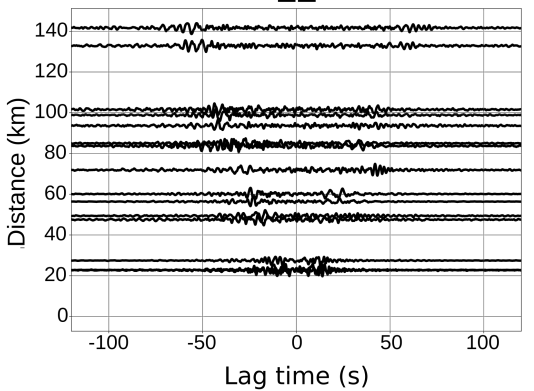

EN

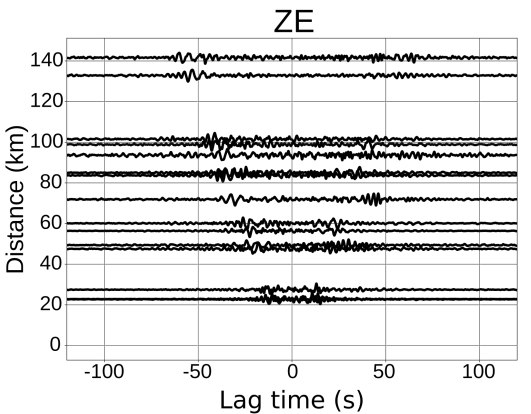

NE
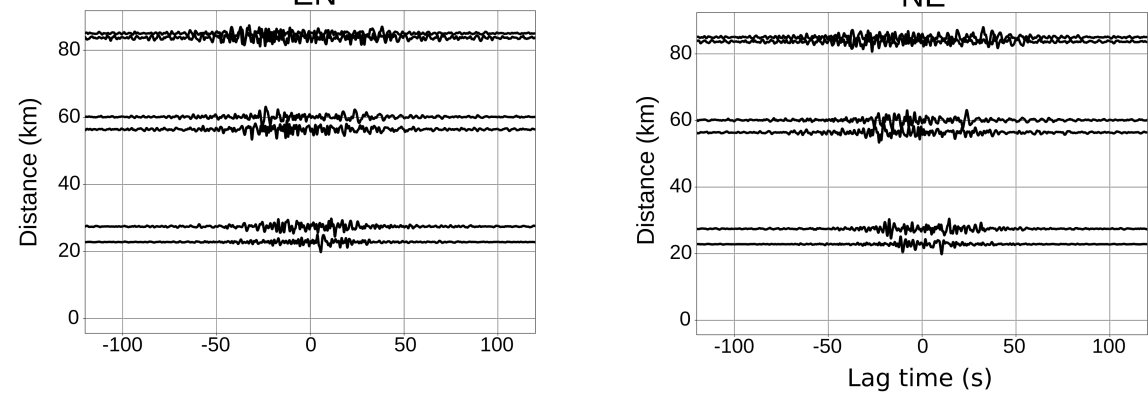

$\mathrm{NZ}$

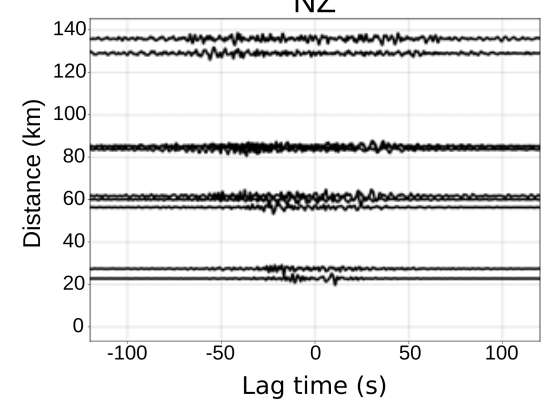

ZN

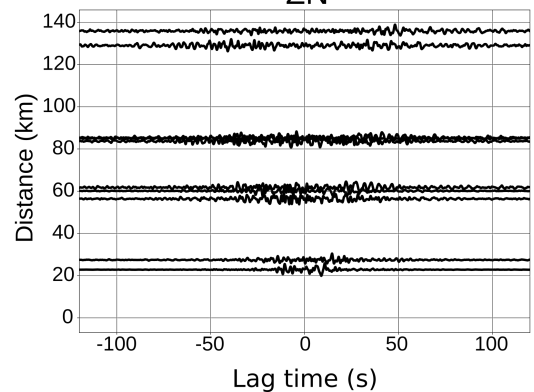

Figure 5.1: Plots of reference cross-correlation functions against interstation distance for all nine component pairs using the seven-year dataset and seven-year reference period. Radial and transverse components were not used. Peaks corresponding to direct arrivals are most visible for pairs not containing a North component. 
5.1 (Stein and Wysession, 2009). Cross-components EZ/ZE and NZ/ZN should also contain Rayleigh waves and components EN/NE and EE should contain both Love and Rayleigh waves.

Energy does not always follow a direct path between stations and this results in scattered arrivals, as shown by the station pairs in Figure 5.1. Despite not having radial and transverse components, either direct or scattered surface wave arrivals are visible in the cross-correlation functions. Another method to check the energy of the arrivals is to plot SNR against lag time. Figure 5.2 shows this using 30-day moving stacks with station pairs that consistently contained data for this duration.

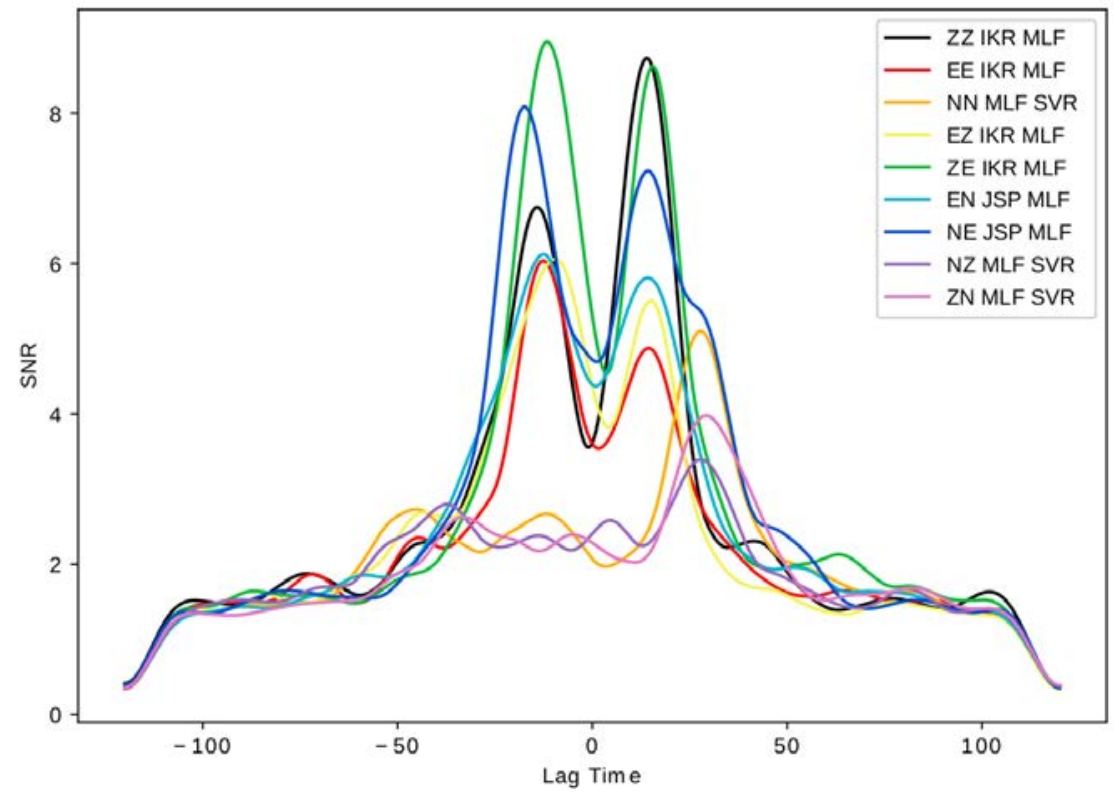

Figure 5.2: Coherent energy is observed for all nine cross-components by plotting the SNR versus lag time for a 30-day cross-correlation function. Different coloured lines indicate different component pairs. All component pairs show peaks at both negative and positive lag times. Component pairs NN, NZ, and ZN show a smaller peak at a negative lag time than other component pairs. These station pairs were used as only these pairs contained data consistently for 30-days to produce SNR values.

Out of the nine component pairs plotted for SNR (Figure 5.2), pairs that include the North component produced the worst result. These are NN, NZ, and ZN as they all have a much lower SNR than the other six pairs. Prominent peaks are observed at positive and negative lag times to reflect the direct arrivals occurring even for the north component pairs. Peaks for different cross-components occur at slightly different times as different station pairs are used as not all stations were 
recording consistently on all components. Interestingly, the amplitude of the negative lag is quite a bit lower than the positive lag, even for ZN where North is not the first component from the station pair. This is possibly a result of data being worse for the North components than other components. As different components have different data availabilities, different cross components are produced from different station pairs. This is why cross components such as ZN and EN can't be directly compared because different stations are being used. However, some pairs can be compared such as ZZ, EE, ZE, and EZ as all cross components use station pair IKR-MLF. It shows that the results from the cross-correlation function for a vertical component were still quite poor when paired with the North component. Despite this, energy within the previously defined window of $15 \mathrm{~s}$ to $55 \mathrm{~s}$ (and $-55 \mathrm{~s}$ to $-15 \mathrm{~s}$ ) is still above the previously determined SNR threshold of 2. As a result of this, all of the cross-components will be used to analyse velocity changes.

\subsection{Velocity Changes for two years}

In this section, the results from the velocity changes using a two-year dataset will be presented.

\subsubsection{Stations Used}

Different stations produce different results, so it is important that only the most reliable stations are chosen. The following stations in Table 5.1 were chosen for the two-year testing period based on correlation coefficient plots, duration and reference stacks. Most of the station choices were discussed in the methods, but a few different stations are used in this section (CVR for EE, and CCB for NN have been removed since being mentioned in the Methods (Table 4.2)). 


\begin{tabular}{|c|c|c|c|}
\hline Component & Stations Used & Reference Period & Figures \\
\hline $\mathrm{ZZ}$ & $\begin{array}{l}\text { IKR, JSP, MLF, SJQ, SVR, } \\
\text { WJM }\end{array}$ & 6-months & $5.1,5.2,5.5,5.6$ \\
\hline $\mathrm{ZZ}$ & $\begin{array}{l}\text { CVR, IKR, JSP, MLF, SJQ, } \\
\text { SRB, SVR, WJM }\end{array}$ & 2-years & $5.3,5.4,5.5,5.6$ \\
\hline $\mathrm{EE}$ & $\begin{array}{l}\text { IKR, JSP, KVR, MHR, } \\
\text { MLF, MTV, SJQ, WJM }\end{array}$ & 6-months & $5.7,5.8 \mathrm{a}$ \\
\hline $\mathrm{NN}$ & $\begin{array}{l}\text { JSP, MHR, MLF, SJQ, SVR, } \\
\text { WJM }\end{array}$ & 6-months & $5.7,5.8 \mathrm{~b}$ \\
\hline EZ & IKR, JSP, MLF, SJQ, WJM & 6-months & $5.7,5.8 \mathrm{a}$ \\
\hline $\mathrm{ZE}$ & IKR, JSP, MLF, SJQ, WJM & 6-months & $5.7,5.8 \mathrm{c}$ \\
\hline $\mathrm{EN}$ & $\begin{array}{l}\text { JSP, MHR, MLF, SJQ, } \\
\text { WJM }\end{array}$ & 6-months & $5.7,5.8 \mathrm{a}$ \\
\hline $\mathrm{NE}$ & $\begin{array}{l}\text { JSP, MHR, MLF, SJQ, } \\
\text { WJM }\end{array}$ & 6-months & $5.7,5.8 \mathrm{~b}$ \\
\hline NZ & JSP, MLF, SJQ, SVR, WJM & 6-months & $5.7,5.8 b$ \\
\hline $\mathrm{ZN}$ & JSP, MLF, SJQ, SVR, WJM & 6-months & $5.7,5.8 \mathrm{c}$ \\
\hline
\end{tabular}

Table 5.1: Stations for the two-year dataset. The table states the component, the stations for that particular component, the reference period used and which figures these stations produced results for.

\subsubsection{Velocity Changes for the $\mathrm{ZZ}$ component}

Velocity changes and their error are calculated for each station pair and then averaged across all station pairs within MSNoise. Velocity changes are first plotted for the vertical-vertical component to determine if any reliable results can be determined. The vertical-vertical component is chosen as the vertical component is more reliable and produces the most stable result for the available data. The Kaikōura earthquake occurred on the $14^{\text {th }}$ of November 2016 and within our two-year dataset, this is the only event that is likely to have caused a significant response. Following the Kaikōura earthquake, the velocity decreased by approximately $0.2 \%$. For the vertical-vertical component, the decrease after the Kaikōura earthquake is visible in the 5-day, 10-day, 20-day, and 30-day moving window velocity variation plots (Figure 5.3).

There is a small lag associated with the change in relative velocity, which is caused by the different moving day window used. For example, with the 10-day moving window, there is a 10-day lag associated, and for the 30-day moving window there is a 30-day lag associated with it. This is because the values are plotted at the end of 


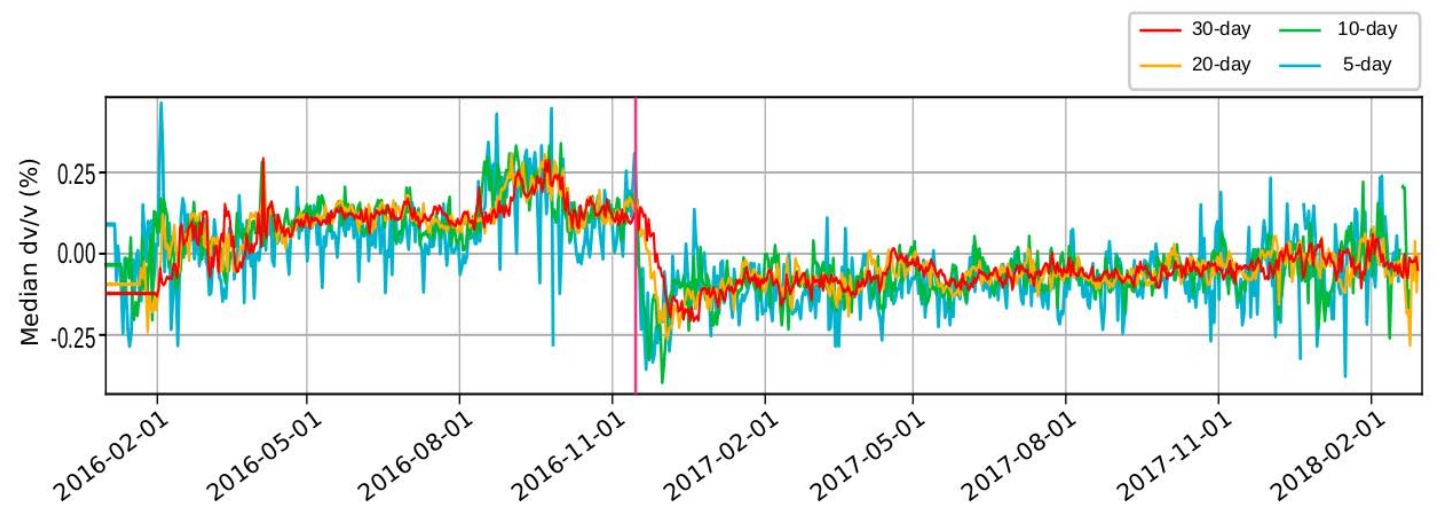

Figure 5.3: Velocity changes using two years of data for the vertical-vertical component for different moving windows, showing the 2016 Kaikōura earthquake (pink line) affecting all of the different moving windows; 5-day (in blue), 10-day (in green), 20-day (in orange) and 30-day (in red). The flat lines at the beginning of each line are different lengths due to different moving windows. The reason for the increase before the earthquake (in October 2016) is currently undetermined. It could be a result of the particular stations chosen (see Table 5.1) or the reference period length.

the windows. The $N$-moving window is the velocity on a given day as the average of the previous $N$-days including itself. A gradual recovery after the earthquake is also visible in this plot. This is most prominent in the 30-day moving window. The 5-day moving window has a high variability as it is the moving window that is most affected by small day-to-day changes. As the moving window increases, these small changes are smoothed out. The most stable result is observed with the 30-day moving window, shown in closer detail in Figure 5.4.

Figure 5.4 shows a median velocity variation decrease of approximately $0.2-0.3 \%$ after the Kaikōura earthquake (shown by the pink line). There is also an associated lag with the 30-day moving window and the lag is greater than other moving windows, due to this period being greater. However, the result is a lot clearer than smaller moving windows, because it has been smoothed over a larger moving window period. There is also a gradual increase after the earthquake.

Different reference periods for the calculations can affect the data. If the reference period includes data that has a sharp decrease and a gradual rise in it, this results in the data before the earthquake being slightly skewed and having higher results. This is shown in the figure (Figure 5.5), which compares two different reference periods 


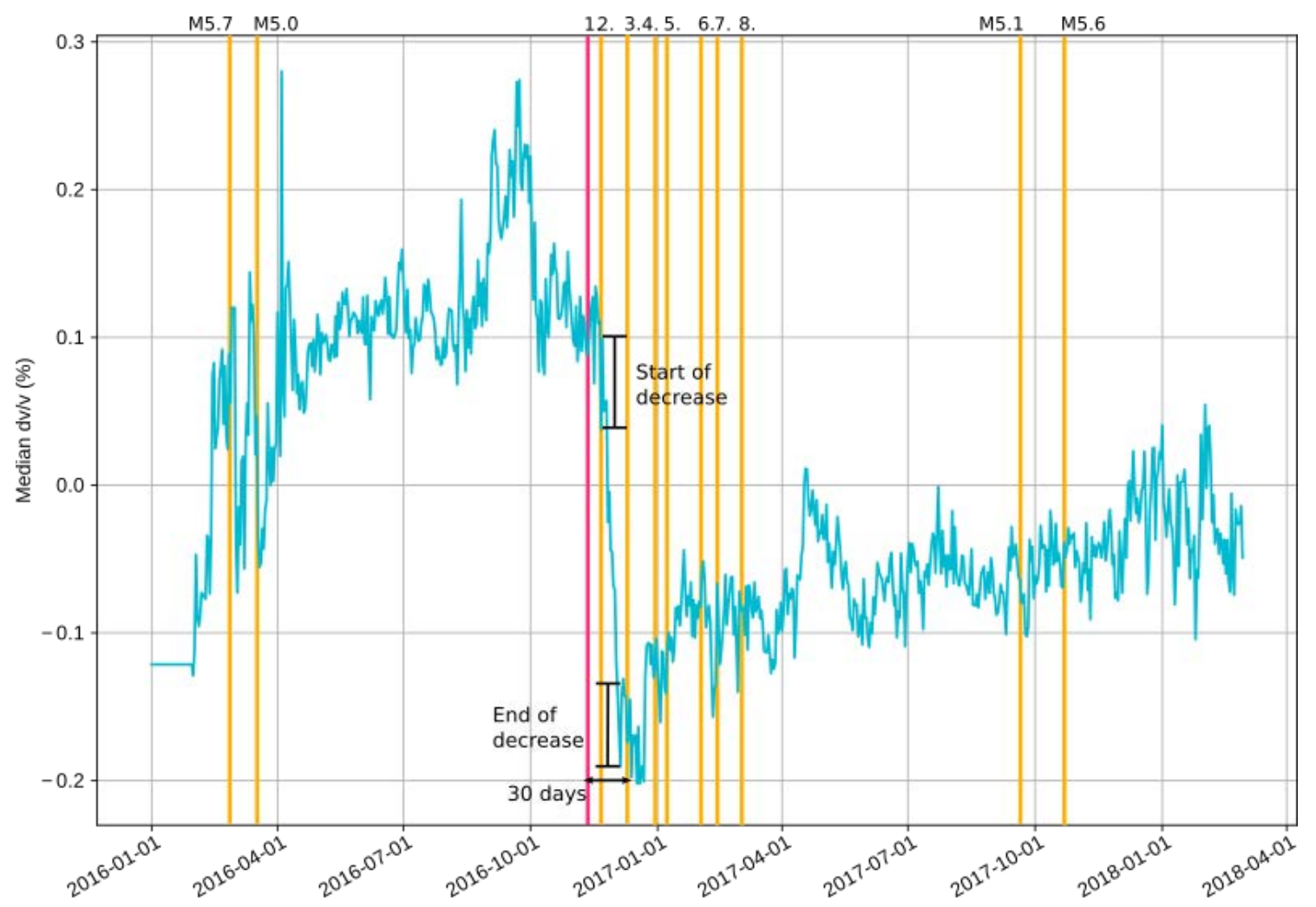

Figure 5.4: Median velocity changes using two years of data for the vertical-vertical component for a 30-day moving window (blue line) with a two-year reference period. The Kaikōura earthquake is shown by the pink line. All other earthquakes with $M_{w}>5.0$ from 2016-01-01 to 2018-02-28 are shown by the orange lines. The numbered orange lines from November 2016 - March 2017, have the following magnitudes: $1=M_{w}$ 7.8/5.8/5.3, $2=M_{w} 5.3 / 5.8,3=M_{w} 5.5,4=M_{w} 5.6,5=M_{w} 5.4 / 5.1,6=M_{w} 5.1$, $7=M_{w} 5.2$ and $8=M_{w} 5.2$. The 46 events with $M_{w}>5.0$ from the $14^{\text {th }}$ of November 2016 to the $15^{\text {th }}$ of November 2016 have not been plotted. The "30-days" indicates the length of the moving window. The start of the decrease and the end is also shown by the arrows. The decrease and increase values were calculated from these midpoints with the errors from either side. All errors are incorporated during the MWCS processing step (Clarke et al., 2011; Lecocq et al., 2014). The flat line at the beginning is due to there not being enough data for the moving window to be averaged yet.

for the same duration of data for different moving windows. In this figure (Figure 5.5), there is a response following the Kaikôura earthquake in all of the moving windows for both the shorter and the longer reference period. The shorter reference period only includes data from before the Kaikōura earthquake ( $1^{\text {st }}$ of January 2016 to the $1^{\text {st }}$ of July 2016). As a result of this, the reference period does not include the main event or any of the aftershock sequence. The longer reference period uses the full two-year dataset and goes from the $1^{\text {st }}$ of January 2016 to the $28^{\text {th }}$ of February 2018. The six-month reference period is not skewed by the large change resulting 

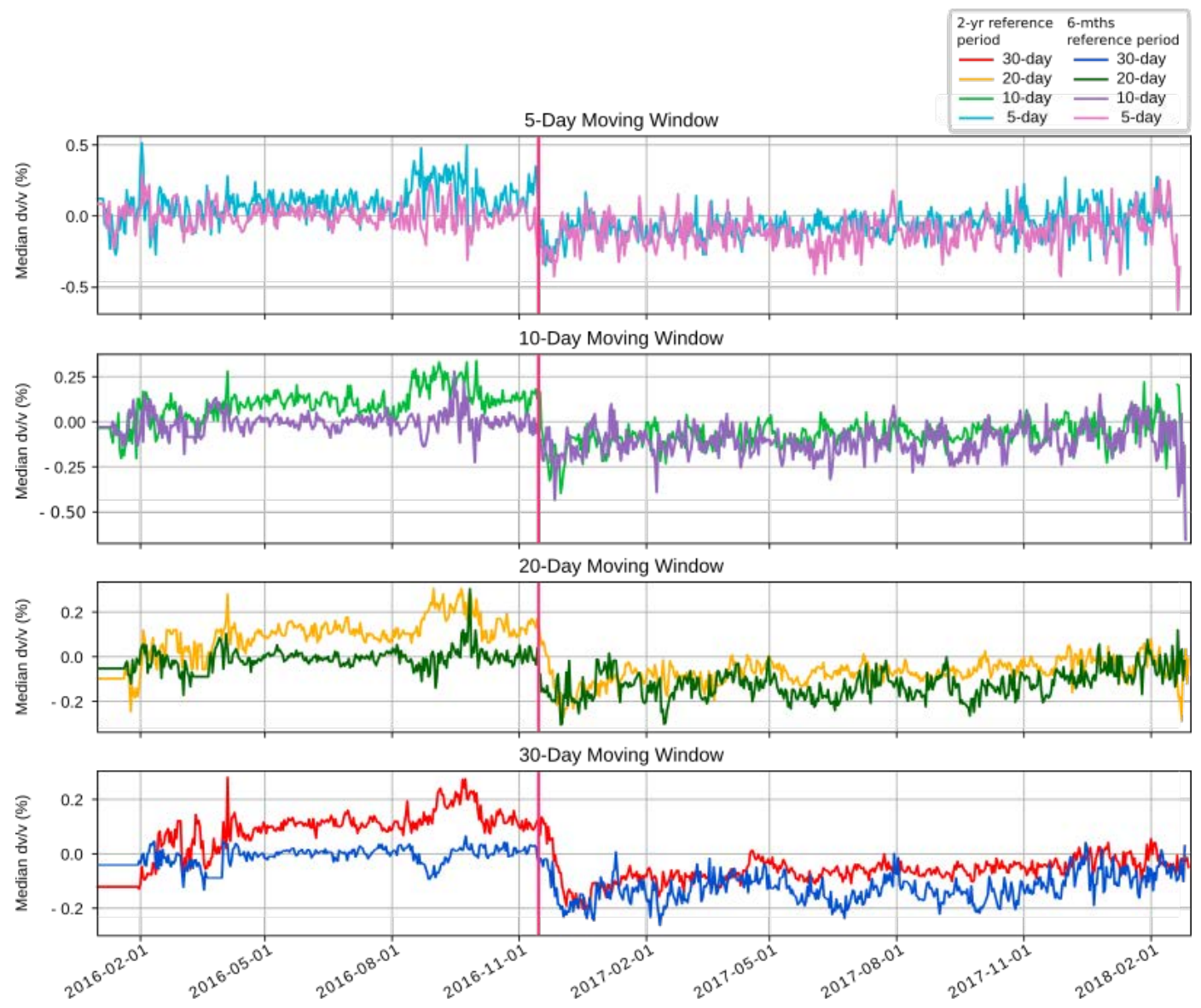

Figure 5.5: Velocity changes using a six month and a two-year reference period for the vertical-vertical component pairs for different moving windows (5-days, 10-days, 20-days, and 30-days). The pink line shows the 2016 Kaikōura earthquake.

from the Kaikōura earthquake, as the two-year reference period is. This results in the median velocity variation values being smaller before the earthquake for the six-month reference period. Despite these higher values, the two-year reference period seems to provide a more stable result, as will be shown when analysing the seven-year dataset. Closer analysis of the 30-day moving window with the Kaikōura earthquake is shown in Figure 5.6.

The 30-day moving window is chosen over the other moving windows (5-day, 10day, 20-day, 60-day and 90-day) because for our dataset it seems to provide the least variability. Longer moving windows of 60-days and 90-days are shown in the Discussion chapter, looking at longer term changes. In Figure 5.6, a blue box is used to illustrate the reference period for the six-month time frame. Using the two-year reference period also seems to give a greater variation in velocity change. With the six-month reference period a decrease of approximately $0.2 \%$ is visible, 


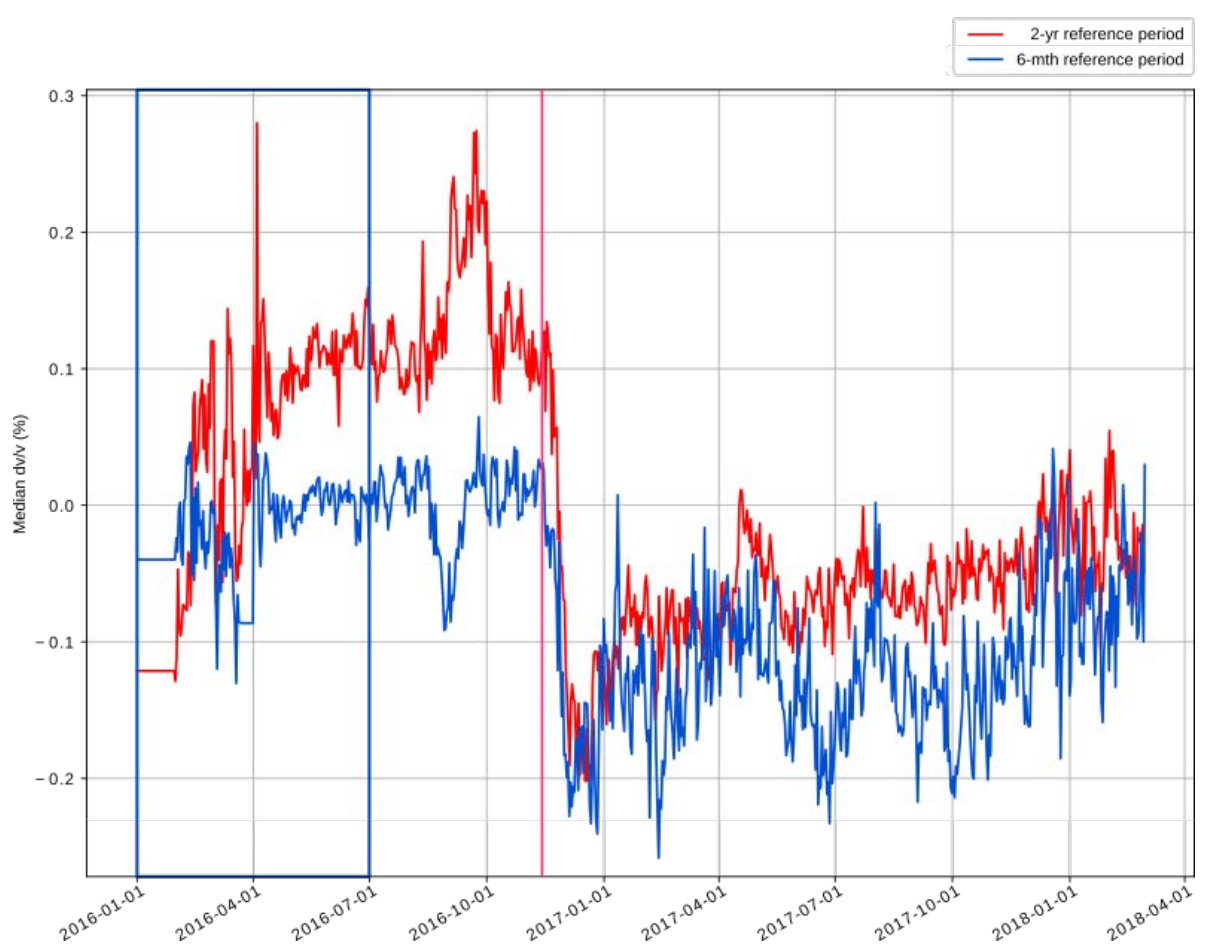

Figure 5.6: Velocity changes using a six-month (blue) and a two-year (red) reference period for a 30-day moving window for the vertical-vertical component. The blue box shows the reference period duration for the six-month reference period. The 2016 Kaikōura earthquake is shown by the pink line. It is uncertain why there is a downward spike between August-October 2016 in the six-month reference period and this is flipped in the two-year dataset.

whereas with the longer reference period, a decrease of approximately $0.2-0.3 \%$ is apparent. This would be because using the two-year reference period includes the Kaikōura earthquake and such a large decrease would skew the overall values and increase the values before the velocity change decrease. With the two-year reference period it also appears to recover more quickly and to appear less scattered than the velocity variations recovered using a six-month reference period. Now that we have a reliable result for the vertical-vertical component it can be applied to the other cross-components pairs.

\subsubsection{Velocity Changes for all cross-components}

The velocity change results for different cross-components produce similar results to the vertical-vertical results shown previously. Figure 5.7 shows all of the crosscomponent pairs for a two-year testing period using a six-month reference period. A 
longer reference period of seven years was used for the seven-year dataset shown in Section 5.3.

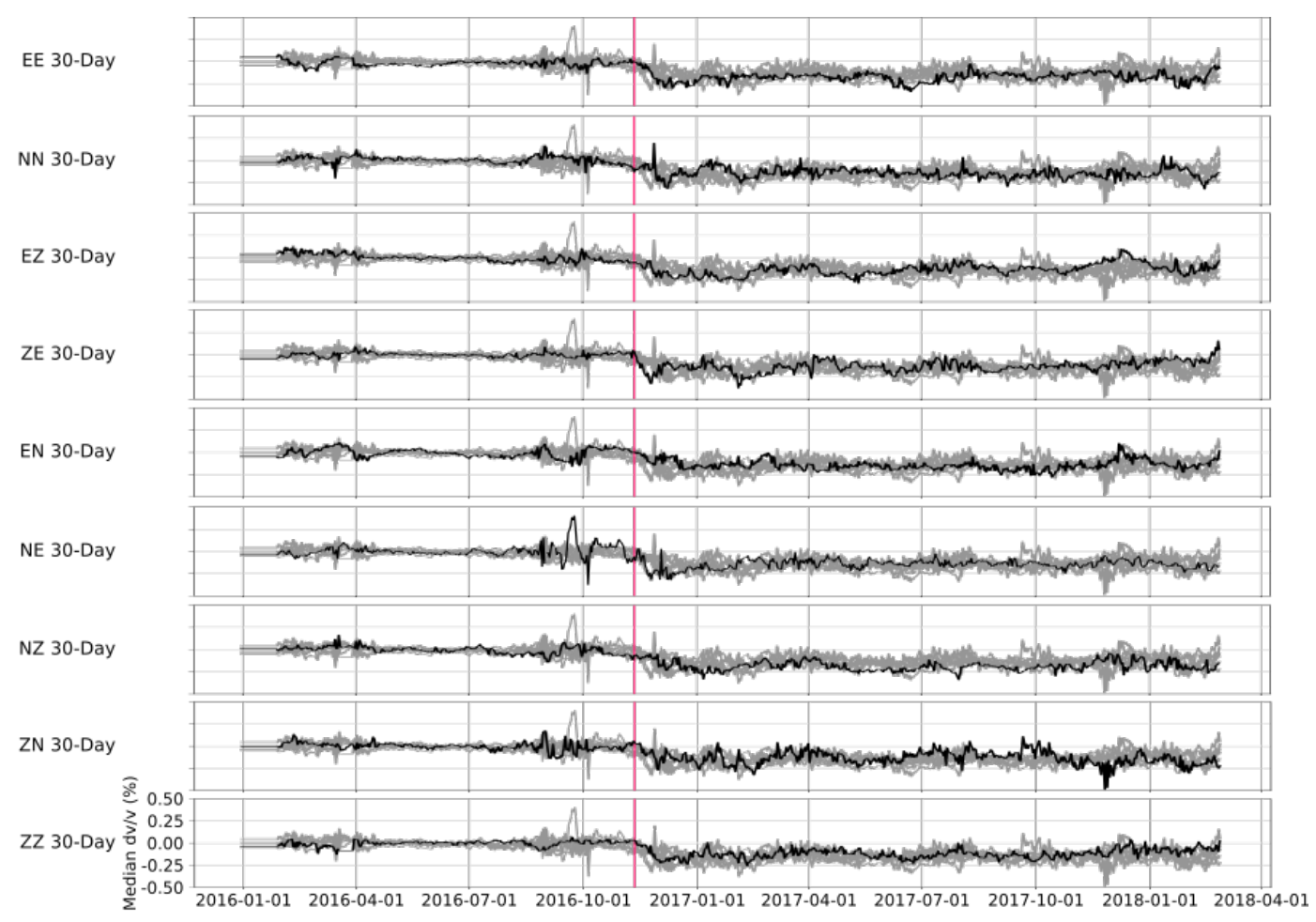

Figure 5.7: Velocity changes for nine different cross-components using a six month reference period for a 30-day moving window. The 2016 Kaikōura earthquake is shown by the pink line. Axes values for cross-components EE-ZN are the same as ZZ.

As shown previously, a 30-day moving window provides the best result, so that will primarily be used to show the different cross-component data. In Figure 5.7, the bottom plot shows the vertical-vertical result and this shows the general trend that all of the other component pairs follow, a sharp decrease just after the Kaikoura earthquake followed by a gradual increase. To more closely analyse the different component pairs, three different figures have been made showing the averages of pairs split by their first station component (Figures 5.8). For example, one figure shows EE, EN, and EZ pairs (Figure 5.8a). Cross-components with the same component combinations would not produce the same result. For example, EN and NE for station pair JSP-MLF would have a different result because JSP (component E) is paired with MLF (component N) and JSP (component N) is paired with MLF (component E). 


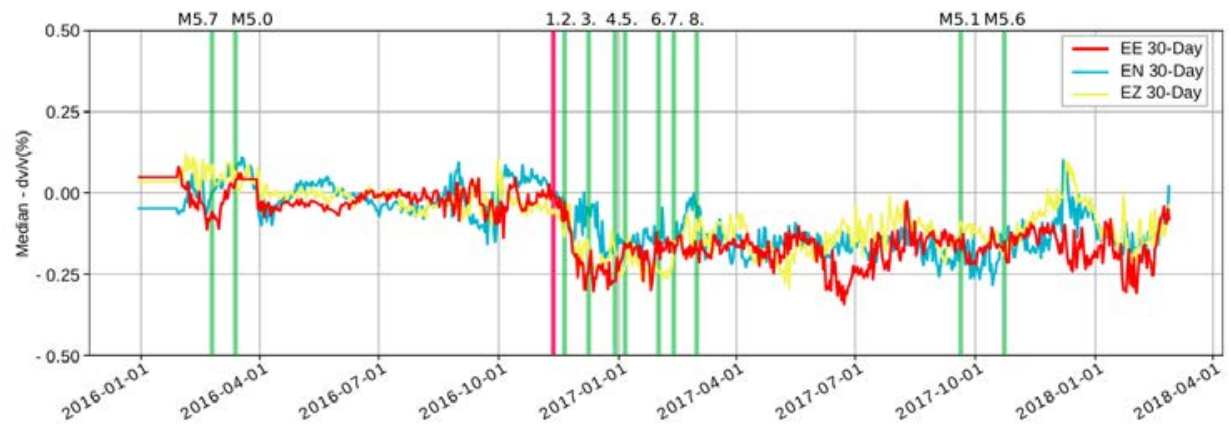

(a) E component pairs

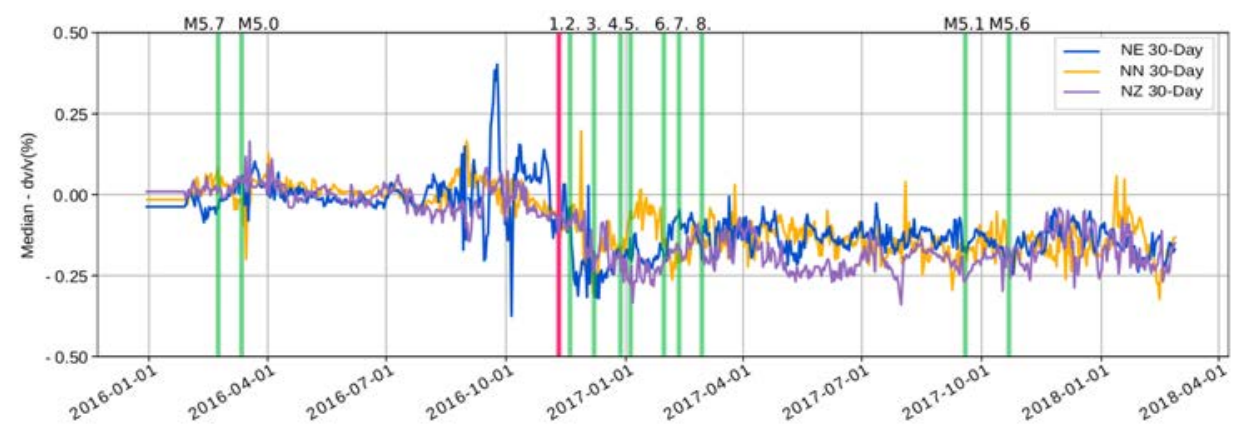

(b) $\mathrm{N}$ component pairs

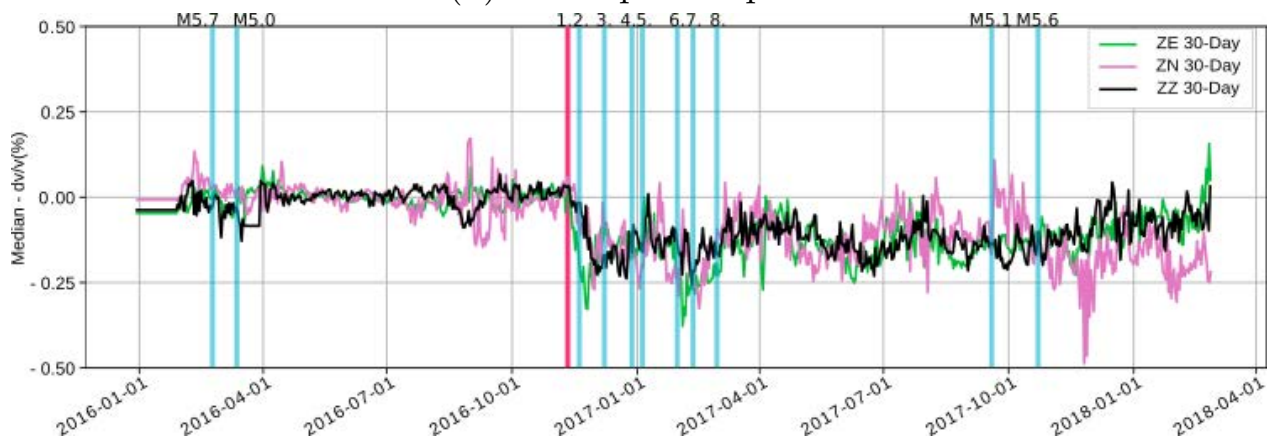

(c) Z component pairs

Figure 5.8: Velocity variation figures for different cross-component pairs for 30-day moving windows with a six-month reference period. a) East components pairs: EE, EN, and EZ. b) North component pairs: NE, NN, and NZ and c) Vertical component pairs: ZE, ZN, and ZZ. The 2016 Kaikōura earthquake is shown by the pink line. All other earthquakes with $M_{w}>5.0$ from 2016-01-01 to 2018-02-28 are shown by the green lines (except for c) which is in blue to distinguish from ZE). The numbered lines from November 2016 to March 2017, have the following magnitudes: $1=M_{w} 7.8 / 5.8 / 5.3,2=M_{w} 5.3 / 5.8,3=M_{w} 5.5,4=M_{w} 5.6,5=M_{w} 5.4 / 5.1,6=M_{w} 5.1$, $7=M_{w} 5.2$ and $8=M_{w} 5.2$. The 46 events with $M_{w}>5.0$ from the $14^{\text {th }}$ of November 2016 to the $15^{\text {th }}$ of November 2016 have not been plotted. 
These figures (Figure 5.8) make it possible to more directly see how each component pair is affected by the earthquake and if they slowly recover or not. Out of the three component pair plots, the East component pairs produce the most stable overall plots with EE providing the best result as this pair is less erratic and pairs using the same component are often more stable. In Figure 5.8a, EE and EZ show a decrease of approximately $0.2-0.25 \%$ whereas EN shows a less clear result. This is potentially a result of the North component contributing erratic results, shown in the distance and SNR plots above (Figures 5.1 and 5.2). The North component pairs (Figure $5.8 \mathrm{~b}$ ) show unstable results, with $\mathrm{NZ}$ or NN potentially producing the best result out of the three. Component pair NE produces an odd spike visible at the beginning of October 2016. This was also clear in the figure showing all of the cross-components (Figure 5.7). This spike changes the velocity variation from approximately $-0.2 \%$ to approximately $0.4 \%$. As this spike is not visible on any other component pairs, it is possible that it is an error in either the stations or in the cross between the North and the East components. The Vertical component pairs (Figure 5.8c) showed generally a good result, with the exception of ZN, which seemed to be more variable and have a few extra increases and decreases whereas component pairs ZE and ZZ did not. This is potentially the North component inducing errors in the component pairs it is a part of. Overall the majority of these pairs produce a stable result and as the two-year dataset produces a good result, it is worthwhile analysing the seven-year dataset to see if there are any other velocity changes prior to 2016.

\subsection{Velocity Changes for seven years}

The results from the two-year data period showed a decrease of approximately $0.2 \pm 0.05 \%$ in the velocity following the Kaikōura earthquake. Due to this, we expand the dataset back to the beginning of 2012 to see if there are any more changes before the Kaikoura earthquake and to see if we have observed any changes due to the 2013 Cook Strait earthquake sequence. To analyse this longer dataset we begin by looking at the stations used so far (Section 5.3.1), then at changes in 
the vertical-vertical component (Section 5.3.2), and then look at the other eight component pairs (Section 5.3.3).

\subsubsection{Stations Used}

Different stations produce different results, so it is important that only the most reliable stations are chosen. Stations were chosen in the same way as mentioned in the Methods Chapter (Section 4.2) and are quite similar to those used for the two-year dataset (Section 5.2). The chosen stations are listed in Table 5.2.

\begin{tabular}{|l|l|l|l|}
\hline Component & Stations Used & Reference Period & Figures \\
\hline ZZ & $\begin{array}{l}\text { CVR, IKR, JSP, MLF, SJQ, } \\
\text { SRB, SVR, WJM }\end{array}$ & 7 -years & $5.10,5.11$ \\
\hline ZZ & $\begin{array}{l}\text { IKR, JSP, MLF, SJQ, SRB, } \\
\text { SVR, WJM }\end{array}$ & 7 -years & 5.11 \\
\hline EE & $\begin{array}{l}\text { CVR, JSP, MLF, SJQ, SRB, } \\
\text { SVR, WJM }\end{array}$ & 2.5 -years & $5.9,5.11$ \\
\hline NN & $\begin{array}{l}\text { CVR, IKR, JSP, KVR, } \\
\text { MLF, MTV, SJQ, WJM }\end{array}$ & 7-years & $5.12,5.13 \mathrm{a}$ \\
\hline EZ & $\begin{array}{l}\text { CCB, JSP, MLF, SJQ, SVR, } \\
\text { WJM }\end{array}$ & 7-years & $5.12,5.13 \mathrm{~b}$ \\
\hline ZE & $\begin{array}{l}\text { CVR, IKR, JSP, MLF, SJQ, } \\
\text { CVR, IKR, JSP, MLF, SJQ, } \\
\text { WJM }\end{array}$ & 7 -years & $5.12,5.13 \mathrm{a}$ \\
\hline EN & JSP, MLF, SJQ, WJM & 7 -years & $5.12,5.13 \mathrm{c}$ \\
\hline NE & JSP, MLF, SJQ, WJM & 7 -years & $5.12,5.13 \mathrm{a}$ \\
\hline NZ & JSP, MLF, SJQ, SVR, WJM & 7 -years & $5.12,5.13 \mathrm{~b}$ \\
\hline ZN & JSP, MLF, SJQ, SVR, WJM & 7-years & $5.12,5.13 \mathrm{c}$ \\
\hline
\end{tabular}

Table 5.2: Stations for the seven-year dataset. The table states the component, the stations for that particular component, the reference period used and which figures these stations produced results for.

Between the two-year and the seven-year results (Tables 5.1 and 5.2), both station choices are similar, except for now, we require stations to have a duration of at least three years instead of one-and-a-half years. One of the few changes was adding CVR for the EE component and adding CCB for the NN component. Both CVR and CCB had a large amount of data for both components before 2016 but not much afterwards, so were not included in the two-year tests. Station MHR was also 
removed from components EE, NN, EN, and NE, because MHR only had data for two-years so did not meet the requirement of a minimum of three years of data for the seven-year dataset.

\subsubsection{Velocity Changes for the $\mathrm{ZZ}$ component}

The first component pair that is analysed in most studies is the vertical-vertical component pair. This pair often shows the most stable result as they receive direct Rayleigh waves. Figure 5.9 shows a plot of a 30-day moving window for the verticalvertical component pair for seven years of data, stacked on the following stations: CVR, IKR, JSP, MLF, SJQ, SRB, SVR and WJM. In this figure there is a clear velocity decrease after the Kaikōura earthquake with irregular and small variations before the earthquake. In the first day of the time series (2012-01-01) there is a large velocity decrease (approximately $0.4 \%$ ), which was seen most strongly on the 30-day moving window for the ZZ component (Figure 5.10). As this decrease was only observed in the first few days and is most prominent in the 30-day moving window, it was confirmed that it was an error in the averaging for the moving day window (an edge effect) and was removed. This edge effect can be observed in Figure 4.27 .

Before the Kaikōura earthquake (Figure 5.9) there is not a gradual increase, (as shown with a larger reference period) but there are a few slight increasing and decreasing pulses. Using a two-and-a-half-year reference period also produces a large spike in the middle of 2015. This was removed when the dataset reference period was increased (see Figure 5.11) and station IKR was added. Upon closer inspection of station pairs used to compute 5.9, shown in Table 5.2, only 10 of the 21 stations pairs has data continuously for the entire reference period (stations CVR, MLF, SJQ, SRB, WJM). The large spike was located a midway through 2015, only three stations (JSP, SJQ, and SVR) had data continuously from the start to the end of 2015, resulting in 3 out of 21 station pairs. Only one of these stations, SJQ, had data in the reference period and for all of 2015. Another station, CVR, was only 


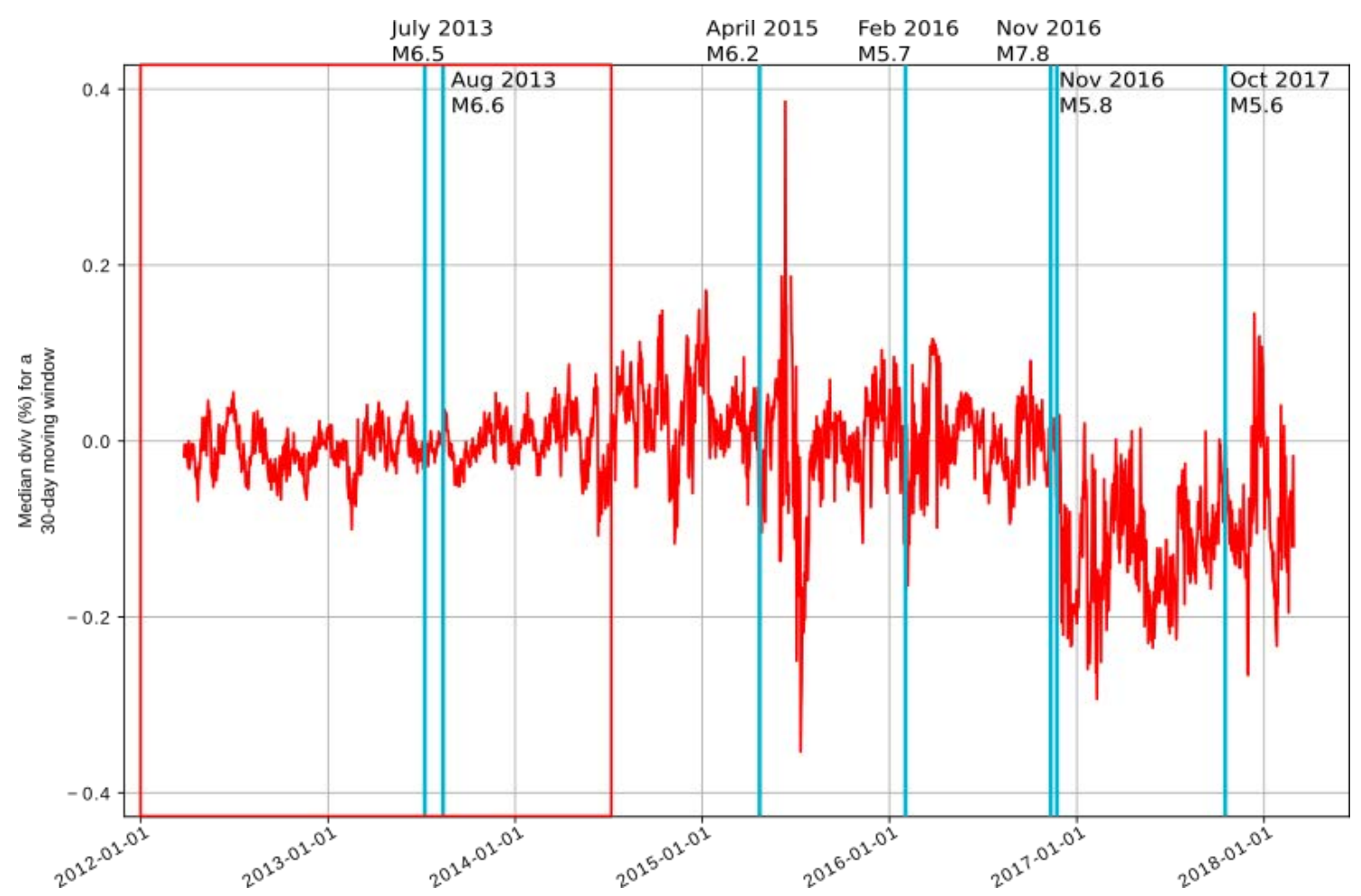

Figure 5.9: Velocity changes for the vertical-vertical component for a 30-day moving window using seven years of data with a two-and-a-half-year reference period (shown by the red box). The 2016 Kaikōura earthquake and earthquakes with $M_{w}>5.6$ are plotted by the blue lines.

missing a few months at the start of 2015, but when included adds 3 more station pairs. When a longer reference period of seven years and station IKR is included (Figure 5.11), four more station pairs can be included that contain data for all of 2015 (CVR-IKR, IKR-JSP, IKR-SJQ, IKR-SVR), this makes 10 out of 28 pairs. Due to the lack of data available in 2015 using a two-and-a-half-year reference period, this spike was visible. As another station was included and the reference period was lengthened, velocity variations in 2015 became smoother and the spike was removed (Figure 5.11).

Viewing the different moving windows allows us to see how the data is affected when more days are averaged (Figure 5.10) for the seven-year reference period $\left(1^{\text {st }}\right.$ of January 2012 to $28^{\text {th }}$ of February 2018). This is especially the case in mid-2015 where there are quite a few fluctuations in the velocity change as there are positive and negative pulses in this period, for the 5-day and the 10-day moving windows (the blue and green lines respectively in Figure 5.10). In the 20-day and 30-day 
moving windows (the orange and red lines respectively) the fluctuations are averaged out. These fluctuations were also visible in the two-and-a-half-year reference period. The 30-day moving window shows the best result as it smooths out all of the daily averages in the velocity variation.

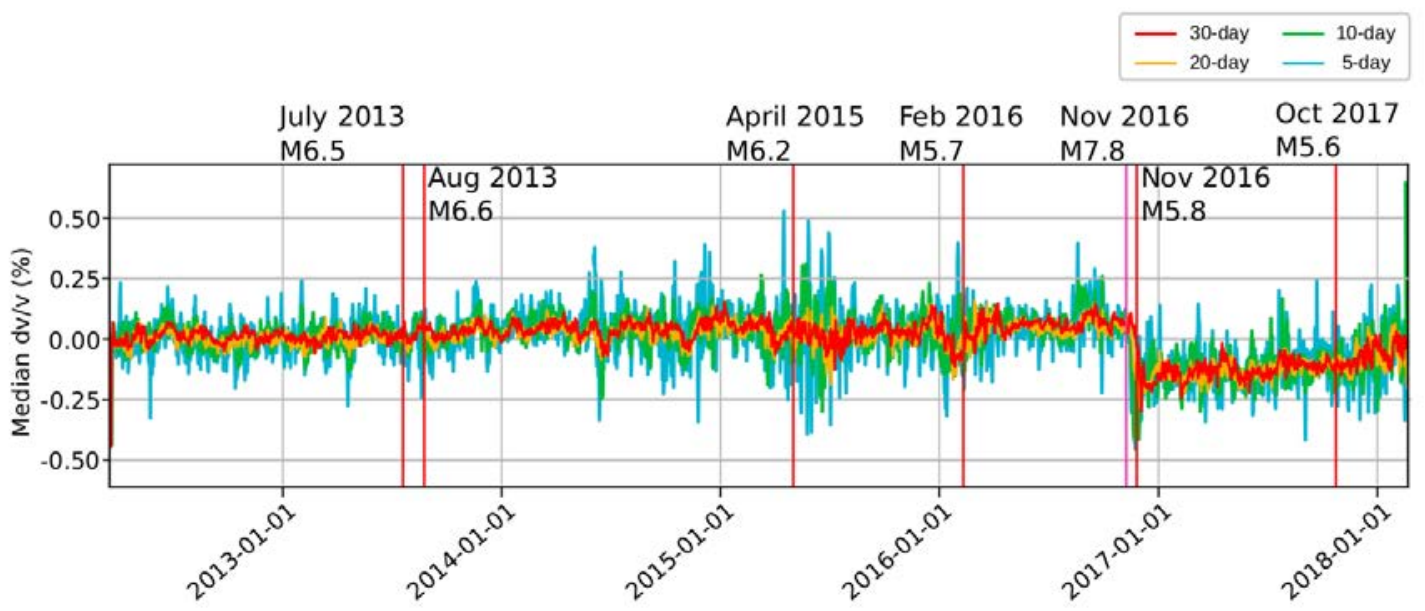

Figure 5.10: Velocity changes for the vertical-vertical component for 5-day, 10-day, 20-day and 30-day moving windows using a seven-year reference period. The red lines indicate large magnitude earthquakes $\left(M_{w}>5.6\right)$ that occurred in this time period. The 2016 Kaikōura earthquake is shown by the pink line.

The stations and the reference period used make a difference. Figure 5.11 shows three different velocity change curves. The results shown in orange and blue use the seven-year reference period and the result shown in red uses a two-and-a-half-year reference period. Although the general pattern of a velocity decrease after the Kaikōura earthquake is robust for all periods, the longer reference period looks more stable. Also shown in this figure is the use of stations CVR, or IKR, or both CVR and IKR, as well as using JSP, MLF, SJQ, SRB, SVR, and WJM. The most noticeable difference in this plot is the large fluctuations of velocity variation changes that occurs in June/July of 2015. This only occurs with the two-and-a-half-year reference period as neither of the seven-year reference period results have it. The two-and-a-half-year reference period runs from the $1^{\text {st }}$ of January 2012 to the $1^{\text {st }}$ of July 2014 , but only contains half of the possible station pairs due to data availability. Large fluctuations are visible in the middle of 2015 due to lack of data for the two-and-a-half-year reference period test as well. These fluctuations are also visible in Figure 5.10 for the smaller moving windows, (the 5-day and the 10-day). It is possible they are 


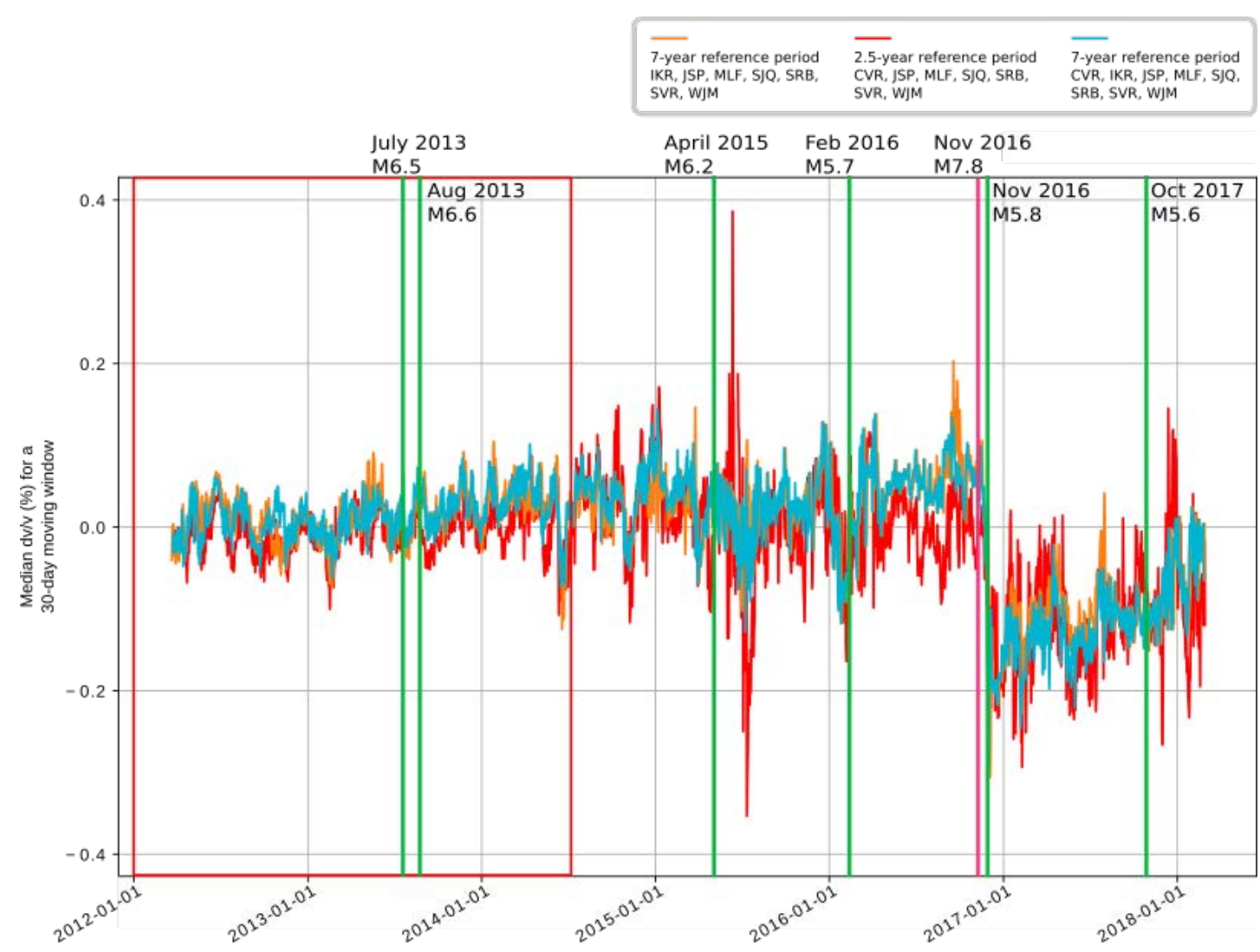

Figure 5.11: Velocity changes for the vertical-vertical component for different stations and reference periods. The red box shows the reference period for the two-and-ahalf-year velocity change results. The 2016 Kaikōura earthquake is shown by the pink line with all other large magnitude events $\left(M_{w}>5.6\right)$ in this time period shown in green. The orange line (without station CVR) is directly underneath the blue line, showing a smoother result (blue line) when CVR is present.

not visible in the 30-day moving windows using the seven-year reference period, because both the seven-year reference period as well as a 30-day moving window give smoother results. To determine the causes of these fluctuations properly, effects of the environment/rain would need to be removed. Due to time constraints this did not occur. In Figure 5.11, in the seven-year reference period, a slight increase before the earthquake in the velocity variation is visible. This is again most likely a result of the large decrease from the Kaikōura earthquake being included in the seven-year reference period but not for the two-and-a-half-year reference period. Station CVR was removed to see if removing one station had a drastic effect on the results. With station CVR (shown in blue) the data seems more coherent than when it is excluded (shown in orange). Removing one station does not change the overall result much, but having more stations with reliable data does produce smoother variations. 
Now that we have coherent results for the vertical-vertical component pair for the seven-year dataset, we analyse all of the remaining cross-component pairs.

\subsubsection{Velocity Changes for all cross-components}

The velocity variations for all nine component pairs produce a similar result to the vertical-vertical seven-year dataset. Figure 5.12 shows all of the cross-component pairs for a seven-year dataset using a seven-year reference period. All cross-components are plotted individually to show that they all show the decrease following the earthquake but are not very similar to each other at different stages.

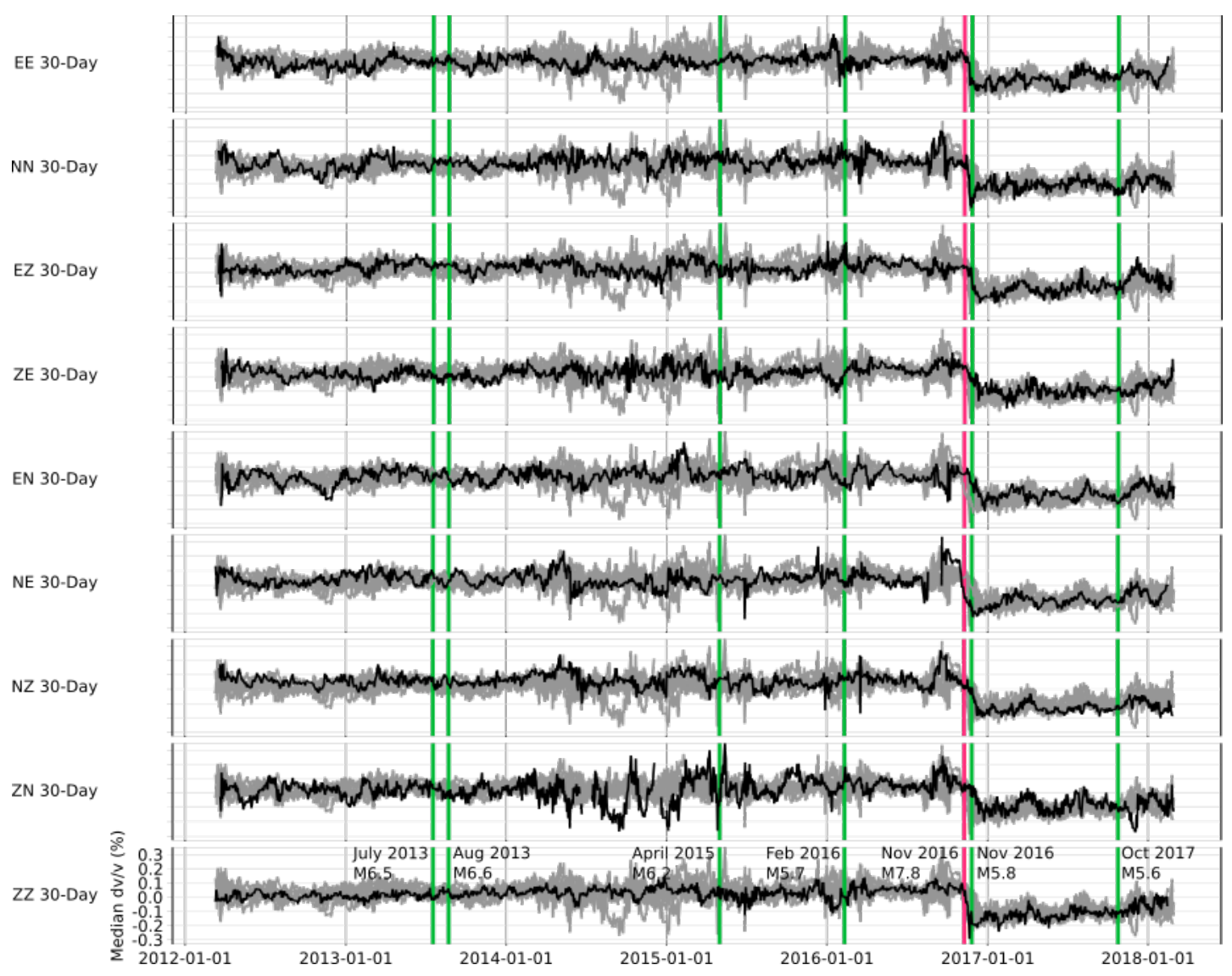

Figure 5.12: Velocity changes for nine cross-component pairs using a seven-year reference period. The 2016 Kaikōura earthquake is shown by the pink line with all other large magnitude events $\left(M_{w}>5.6\right)$ in this time period shown in green. Axes values for cross-components EE-NZ are the same as ZZ.

Once again, the 30-day moving window produces the best result so only the 30-day moving window results are used to show the different cross-component data. In Figure 5.12 the bottom plot is used again to show the vertical-vertical result. The 
vertical-vertical result shows the general trend that all of the other components are following: a sharp velocity decrease just after the earthquake followed by a gradual velocity increase. Before the 2016 Kaikōura earthquake, the velocity variation only had small changes (approximately $0.075 \%$ from peaks to troughs) with a few larger velocity decreases during mid-2015 and at the beginning of 2016 (both had decreases of approximately $0.1 \%$ ). After these decreases, the velocity variation quickly (within a month) returns to equilibrium, implying that these changes are small, most likely not caused by a major event but by fluctuations caused by differing values at different stations. To more closely analyse the different component pairs, three different figures have been made showing the pairs split by their first station component (Figures 5.13), as shown previously for the two-year dataset.

Figures 5.13 shows more directly how each component pair is affected by the earthquake and if they slowly recover or not after the Kaikōura earthquake. Beginning with the East component pair plot (Figure 5.13a), component pair EE produces one of the clearest overall results with a velocity decrease of approximately $0.2 \%$ following the Kaikōura earthquake. There is also a rapid velocity increase and decrease of approximately $0.1 \%$ in the beginning of 2016 . This is quite similar to one of the peaks found in the ZZ plot shown in Figures 5.12 and 5.13c. The EE component also starts quite high with a velocity variation of $0.2 \%$. As this component pair as well as all of the others started with a value of either $-0.1 \%$ or $0.1 \%$ or greater, this seems to imply that within the first few days of the 30-day moving window, the values are not very accurate and so were removed. As moving stacks use days prior to the date of the plotted day to calculate the stacked values, changes are plotted later with a longer moving window. As we are using a 30-day moving window, the velocity changes following the Kaikōura earthquake will not be fully averaged until the $14^{\text {th }}$ of December as, on this day, the 30-day moving average will contain velocity changes from the $14^{\text {th }}$ of November to the $14^{\text {th }}$ of December. Component pair EN produces a fairly similar trend to other cross-component pairs, but it shows a velocity pulse where EN has a velocity increase of approximately $0.25 \%$ at the beginning of 2015 before rapidly (over a month) returning to equilibrium. The increase however 


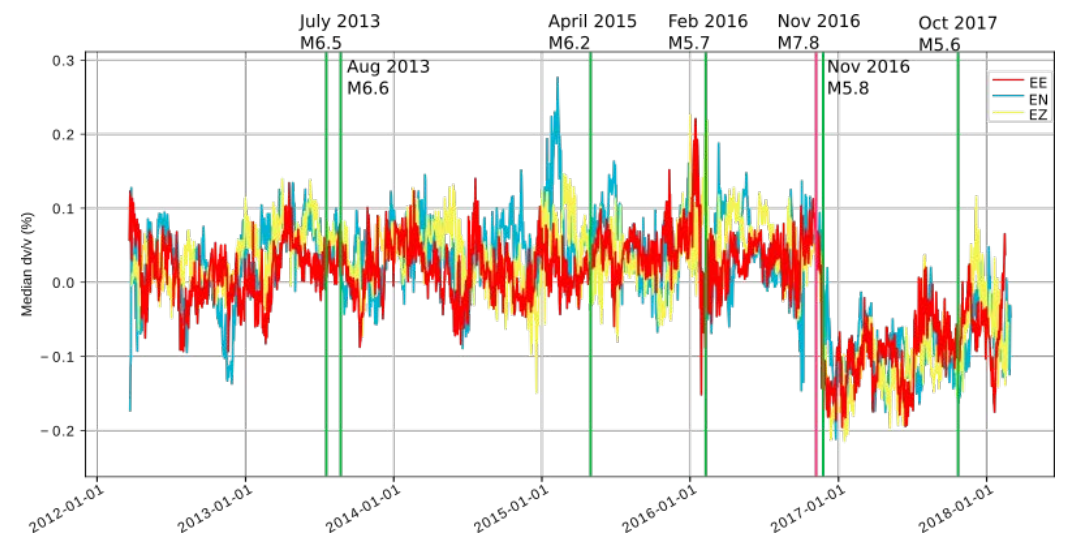

(a) E component pairs

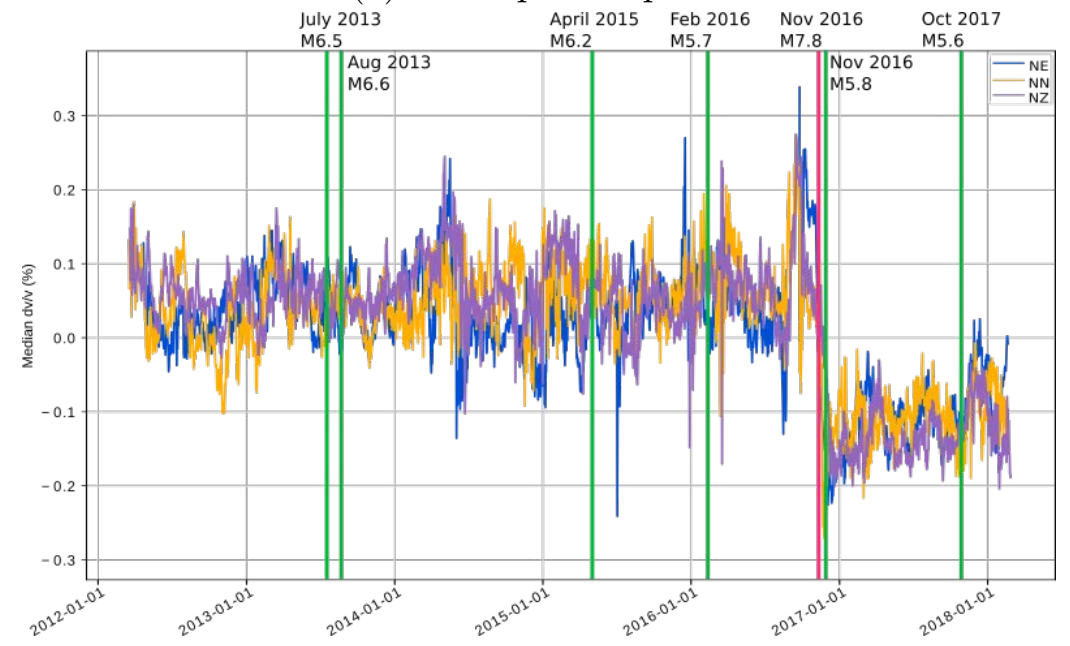

(b) $\mathrm{N}$ component pairs

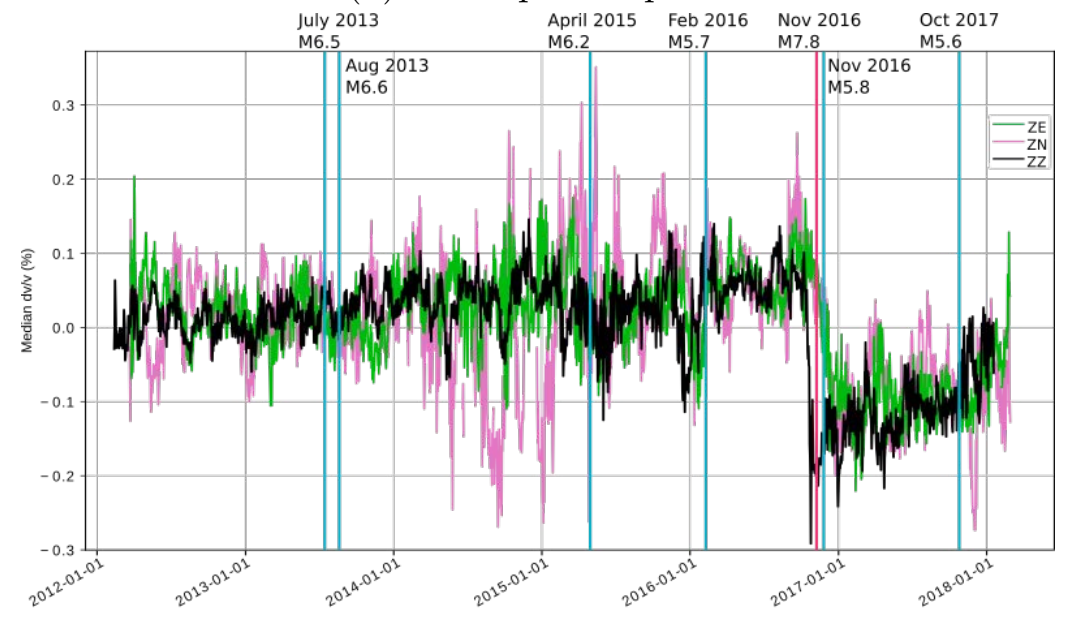

(c) Z component pairs

Figure 5.13: Velocity variation figures for different cross-component pairs for 30-day moving windows for a seven-year dataset with a seven-year reference period. a) East components pairs: EE, EN, and EZ. b) North component pairs: NE, NN, and NZ and c) Vertical component pairs: ZE, ZN, and ZZ. The 2016 Kaikōura earthquake is shown by the pink line with all other large magnitude events $\left(M_{w}>5.6\right)$ in this time period shown in green (except for c) whereas the other faults are shown in blue to distinguish from the ZE component). 
does not affect the overall trend. Component pair EZ is similar to both EE and EN with only an additional decrease at the end of 2014 of approximately $0.15 \%$. The E-component pairs show the same overall trend despite small, sharp increases and decreases in velocity.

The N-component pairs (Figure 5.13b) show a much more varied result than the other cross-components. From the distance and SNR plots shown earlier (Figures 5.1 and 5.2), any cross-component that contained the north component produced a result where the direct arrivals were less visible and in the SNR plots the reference stacks produced low SNR values; values just above 2. From these figures we determine that any result that contained the north component would not be as clear as other cross-components. Out of the three cross-components shown in Figure 5.13b, the NZ component produces the most stable result followed by the NN component. The NZ component contains data from the vertical component, which has already been shown to produce reliable changes in velocity, which is potentially in this situation improving the quality of the north component. The NZ component shows a clear velocity decrease of approximately $0.2 \%$ following the 2016 Kaikōura earthquake with velocity increases in mid-2014 and another increase a month before the 2016 Kaikōura earthquake. The NN component produces a very similar result to the NZ component with the exception of having slightly greater velocity increases and decreases at the same intervals. The NE component shows a large velocity decrease following the 2016 Kaikōura earthquake (approximately $0.3 \%$ ) and also has a few very sharp increases and decreases in velocity. These occur at the beginning of the time period at the start of 2012 (increase of $0.4 \%$ ), decrease in mid-2014 (approximately $0.15 \%$ ), decrease in mid-2015 (approximately $0.25 \%$ ) and a velocity decrease and increase before the 2016 Kaikōura earthquake (approximately $0.1 \%$ and approximately $0.3 \%$ ). The N-component pair shows the same overall trend as the other components, but tends to have a few more outliers that would affect the overall result if all of the pairs were merged together.

The Z-component pairs (Figure 5.13c) show both the most stable and potentially 
the most erratic results. The ZZ component shows a very clear result with a velocity decrease of approximately $0.25 \%$ following the 2016 Kaikōura earthquake. This component pair also shows a large velocity decrease at the beginning of the dataset of approximately $0.4 \%$, as this was an edge effect, the first few days of data were removed. This edge effect only seems to have affected the 30-day moving window, as it does not appear, or minimally appears in the smaller moving windows (see Figure 5.10). The ZZ component pair also shows a gradual velocity increase of $0.2 \%$ over one-and-a-half-years with changes in velocity gradually recovering to similar values or close to those before the 2016 Kaikōura earthquake. The ZE component pair shows a velocity decrease of approximately $0.2 \%$ and no great fluctuations before the Kaikōura earthquake. This pair also follows the trend of the ZZ component pair with a gradual velocity increase following the 2016 Kaikōura earthquake. The final component pair, ZN, produces potentially the least stable velocity changes with large increasing and decreasing pulses in mid-to-late 2014 and early-to-mid 2015. These fluctuations are on the order of $0.25-0.35 \%$ and if all of the cross-components were combined would drastically skew the final result.

Overall the majority of the the cross-component pairs produce a stable result and show a decrease of approximately $0.2 \%$ following the 2016 Kaikōura earthquake. However, as the results produced from all of the cross-components are quite varied, it has been decided that for closer analysis of depth and spatial changes across the region, the results of all nine cross-components will not be combined and only the ZZ component pair will be used. The ZZ component produces the best and most stable result and while it is interesting to see what results the other cross-components produce, as they can not be rotated to produce radial and transverse components (due to stations lacking both horizontal components for the same period of time), the cross-components will all be kept separate. 


\section{Chapter 6}

\section{Discussion}

This chapter is divided into three sections. The first section investigates the depth sensitivity of the results using filter analysis and depth sensitivity kernels, and also analyses how distributed the results are across the northern South Island (Section 6.1). The next section discusses the overall result of the 2016 Kaikōura earthquake and if any changes were discerned from the 2013 Cook Strait earthquake sequence and from two large earthquakes in 2015 and early 2016 (Section 6.2). Mechanisms that could have caused the velocity changes in association with the Kaikōura earthquake will also be discussed within this section. In Section 6.3, previous studies will be used to compare to our velocity change results and determine whether processes suggested from previous studies can be invoked here. Finally in Section 6.4, we conclude by discussing the potential for real-time monitoring and how ambient noise can be used to better understand geologic hazard.

Several possible mechanisms for velocity change associated with the 2016 Kaikōura earthquake will also be analysed later in this chapter. Four main mechanisms have been analysed by Xu and Song (2009) and Wegler et al. (2009) and will be analysed to see if they are likely to be the cause of the velocity changes that have occurred for this dataset. These four mechanisms are 1) stress-induced changes of fault zone properties at seismogenic depth, 2) damage of the crust from fault zone rupture and subsequent healing, 3) damage of the shallow crust from strong-ground shaking, and 
4) rapid changes in groundwater near the surface or fluid activities in the shallow crust. To determine the appropriate mechanism driving the observed velocity changes analysis of the data, methods and the earthquake causes must be considered. It is also important to highlight that velocity variation results display the most prominent mechanism (Wegler et al., 2009). A few of these different mechanisms will be shown in Section 6.2.4.

\subsection{Sampling Depth and Location}

In this section, different frequency ranges are analysed to see if there are any velocity variations with depth (Section 6.1.1). A depth sensitivity kernel has been generated to relate the group velocity values to depth using a New Zealand velocity model. Different station pair combinations will be analysed across the Kaikōura region and the northern South Island to see if spatial variations make any difference in the final velocity results (Section 6.2.1).

\subsubsection{Depth Sensitivity Kernels}

Surface waves are sensitive to depth and as such can be used to determine the depth of penetration in ambient noise studies. To determine the depth of observed changes, depth sensitivity kernels are one of the most accurate ways to determine these results. Depth sensitivity kernels use the theory that the velocity of surface waves can be considered directly proportional to shear wave velocity (Hobiger et al., 2012). From this, codes written by Herrmann (2013), take the derivative of fundamental mode Rayleigh and Love wave group velocities with respect to shear wave velocities, for different periods, which then gives depth sensitivity kernels. The group velocities are calculated using a velocity model developed for a particular region of interest.

Before generating a depth sensitivity kernel, four different frequencies are used to plot the results of velocity variation using nine stations: CVR, IKR, JSP, KVR, 
MLF, SJQ, SRB, SVR and WJM. The four filters used are: $0.1-0.25 \mathrm{~Hz}, 0.25-0.5 \mathrm{~Hz}$, 0.5- $0.9 \mathrm{~Hz}$ and the frequency range that has given us the most stable result so far, 0.1-0.9 Hz. These are plotted in Figure 6.1 using a time period of seven-years with a reference period of seven-years for the vertical-vertical component.

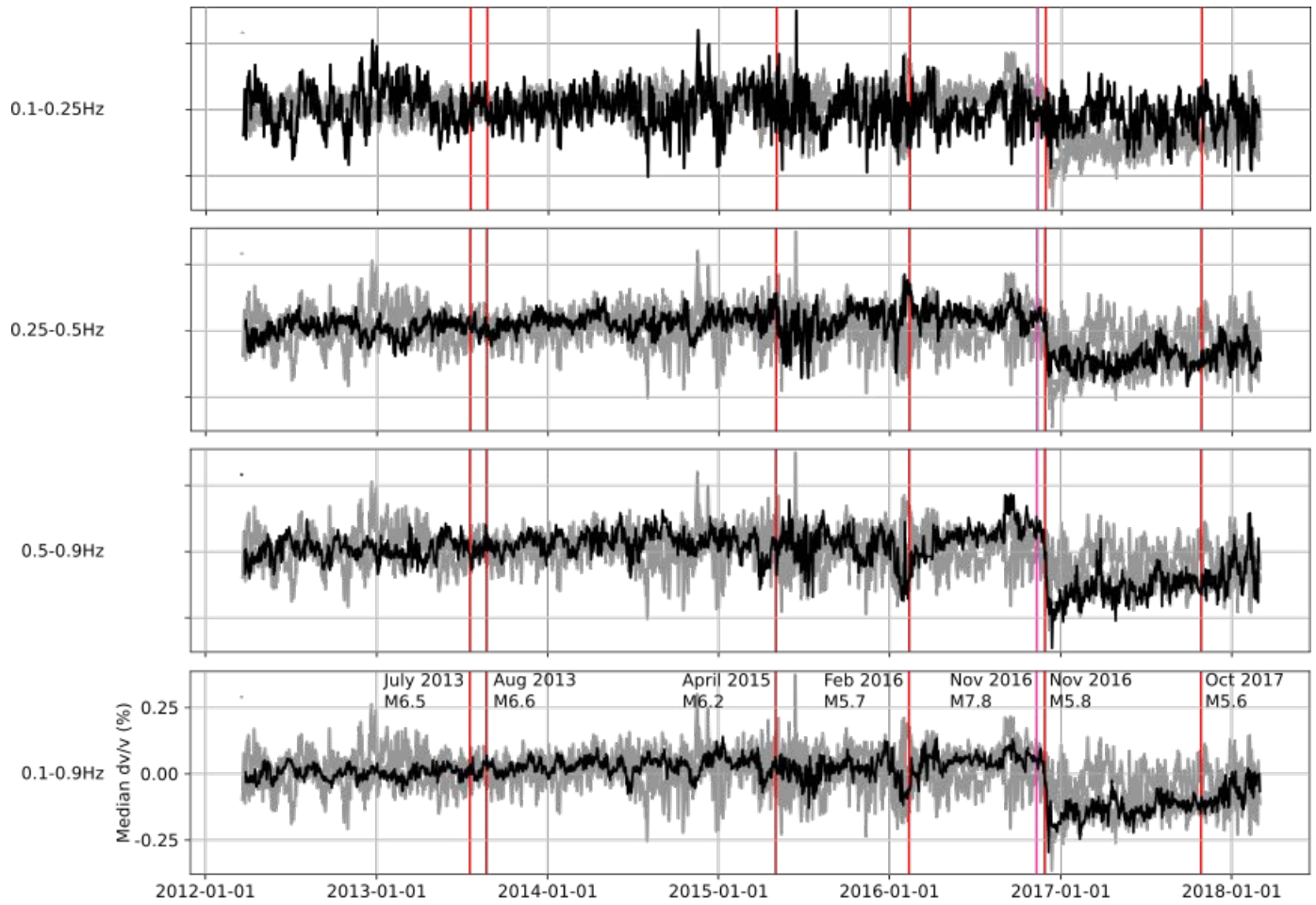

Figure 6.1: Velocity variation plot for four different frequency ranges: $0.1-0.25 \mathrm{~Hz}$ (at the top), $0.25-0.5 \mathrm{~Hz}$ (second), $0.5-0.9 \mathrm{~Hz}$ (third) and $0.1-0.9 \mathrm{~Hz}$ (at the bottom). This is plotted using a 30-day moving window for the vertical-vertical component over a seven-year duration with a seven-year reference period. The pink line shows the 2016 Kaikōura earthquake. The red lines show all large magnitude $\left(M_{w}>5.6\right)$ earthquakes. Axes values for the first three plots are the same as the bottom plot.

From the results shown in Figure 6.1, all of the filters except for the 0.1-0.25 Hz filter (the lowest frequency filter) show a decrease in the velocity after the 2016 Kaikoura earthquake. The $0.1-0.25 \mathrm{~Hz}$ filter shows the deepest results, while the other filters $(0.25 \mathrm{~Hz}$ and greater), show shallower results. The $0.1-0.25 \mathrm{~Hz}$ frequency range does not match any of the trends shown in the three other frequency ranges, so this suggests that the depth of sensitivity is too deep for velocity changes to be observed. Above $0.25 \mathrm{~Hz}$ (second plot in Figure 6.1), there is a velocity decrease of $\sim 0.2 \%$ following the Kaikōura earthquake. The frequency range above the $0.25-0.5 \mathrm{~Hz}$ range, $0.5-0.9 \mathrm{~Hz}$ (third plot in Figure 6.1) also shows a velocity change following the earthquake. The clearest result is observed with the frequency range $0.1-0.9 \mathrm{~Hz}$, 
as it shows the clearest velocity decrease and appears less scattered than the other waveforms. As ambient noise changes resulting from the Kaikōura earthquake only appear above $0.25 \mathrm{~Hz}$, this suggests that frequency ranges lower than this (and thus deeper) are not recording any changes. As we now have an understanding of what frequency ranges we would no longer expect to result in a change in velocity, we are therefore able to generate a depth sensitivity kernel to more precisely determine the depth of resolution.

Surface waves (Rayleigh and Love waves) can be used to calculate changes in depth because they change velocity with period, and greater periods extend to deeper depths. Rayleigh wave energy is arriving on the vertical-vertical component, so a depth sensitivity kernel will be generated with the Rayleigh fundamental modes. These can be directly correlated to the results in Figure 6.1, which were generated using the vertical-vertical component.

Depth sensitivity kernels are obtained by taking the derivative of the fundamental Rayleigh wave group velocities with respect to shear wave velocities, computed for different periods using group and phase velocities (codes by Herrmann (2013)). Group velocities are calculated using the New Zealand velocity model by Eberhart-Phillips and Fry (2018). Depth, Vp, Vs, and density values were used from the velocity model for the ruptured region of -41 to -43 latitude and 171 to 175 longitude (Figure 1.4). These results were only calculated to depths of $25 \mathrm{~km}$, as deeper than this, no changes in sensitivities were observed. Depth sensitivity kernels are shown in Figure 6.2 for a range of periods in the dataset in this thesis. The dataset is sensitive to a wide range of depths, with the shortest periods sensitive to the upper $5 \mathrm{~km}$. This corresponds to the results shown in Figure 6.1, as the longer periods $(4 \mathrm{~s}, 5 \mathrm{~s}$, and $10 \mathrm{~s}$ ) and therefore lower frequencies have higher partial derivatives for deeper values and therefore observe deeper changes than higher frequencies. As the velocity decrease after the Kaikōura earthquake is not seen in the $0.1-0.25 \mathrm{~Hz}$ frequency range (Figure 6.1), then from Figure 6.2, the deepest depth to observe the velocity changes due to the Kaikōura earthquake is $2.5-5 \mathrm{~km}$. 


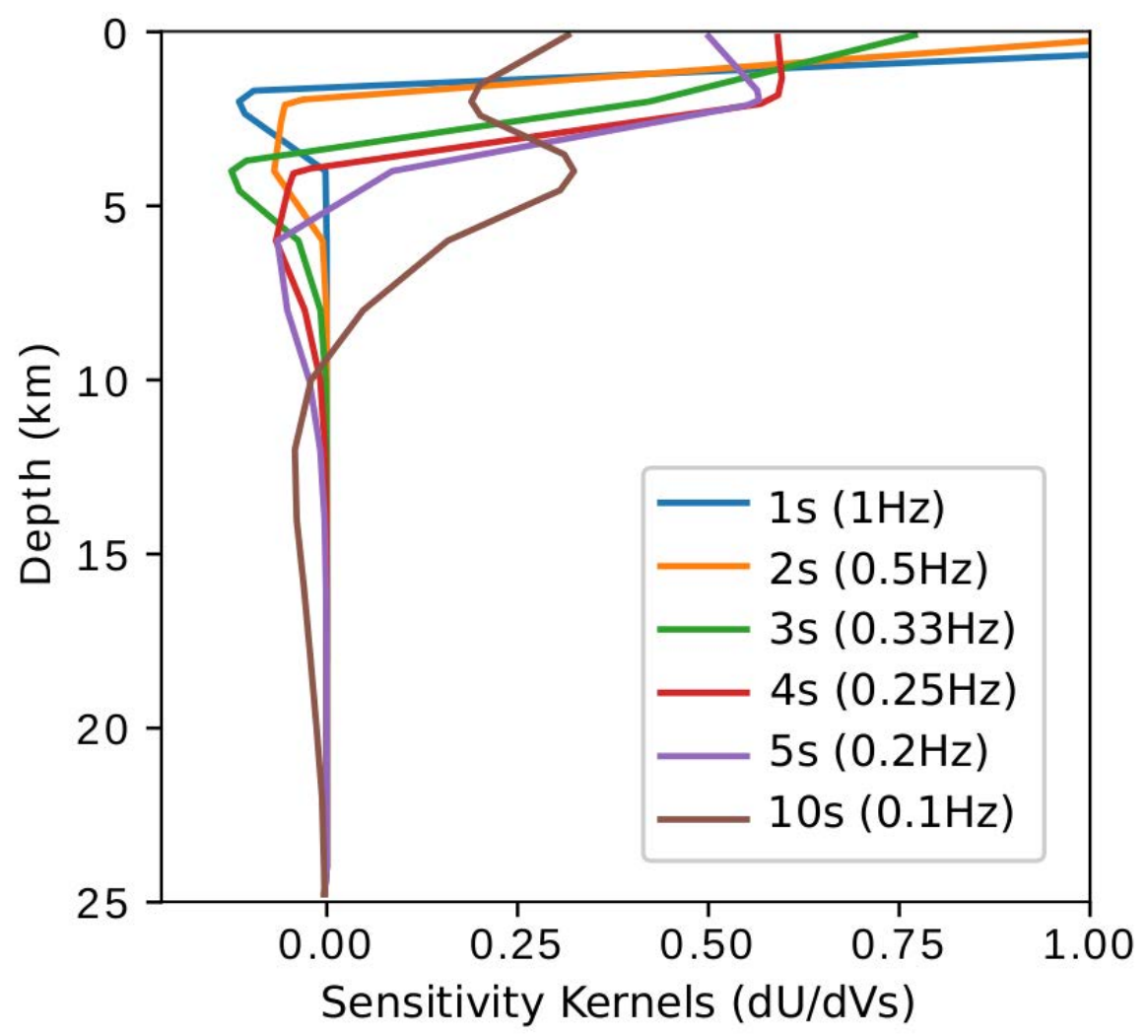

Figure 6.2: Depth sensitivity kernels computed for the fundamental mode Rayleigh wave group velocity with respect to the shear-wave velocity at periods corresponding to the minimum and maximum periods of the dataset used in this thesis. The derivatives are based on the average regional velocity model (Eberhart-Phillips and Fry, 2018). The result at $0.1 \mathrm{~Hz}$ does seem rather odd compared to the other values, but this is only because of the gap between $5 \mathrm{~s}$ and $10 \mathrm{~s}$. Other papers have also found a similar result for $0.1 \mathrm{~Hz}$ (e.g., Nimiya et al., 2017; Heckels, 2017).

\subsubsection{Velocity Changes across the South Island}

Another important consideration is how the ambient noise results change depending on the distance away from the Kaikōura earthquake, and how the different faults in the region are affected. To analyse different station pairs across the Kaikōura region, two years of data for the vertical-vertical component were analysed. A two-year period was chosen as the main aim was to focus on velocity changes only related to the Kaikōura earthquake. To begin with, the region was split into four separate regions; northeast, southeast, southwest and northwest, where the North-South line runs parallel to the direction of the South Island (roughly $30^{\circ}$ East of True North). The east-west line runs perpendicular to the north-south line in the middle of the station map (Figure 6.3). These divisions split the region that the stations occupied 
equally and also had the north-south line running parallel to the coast.

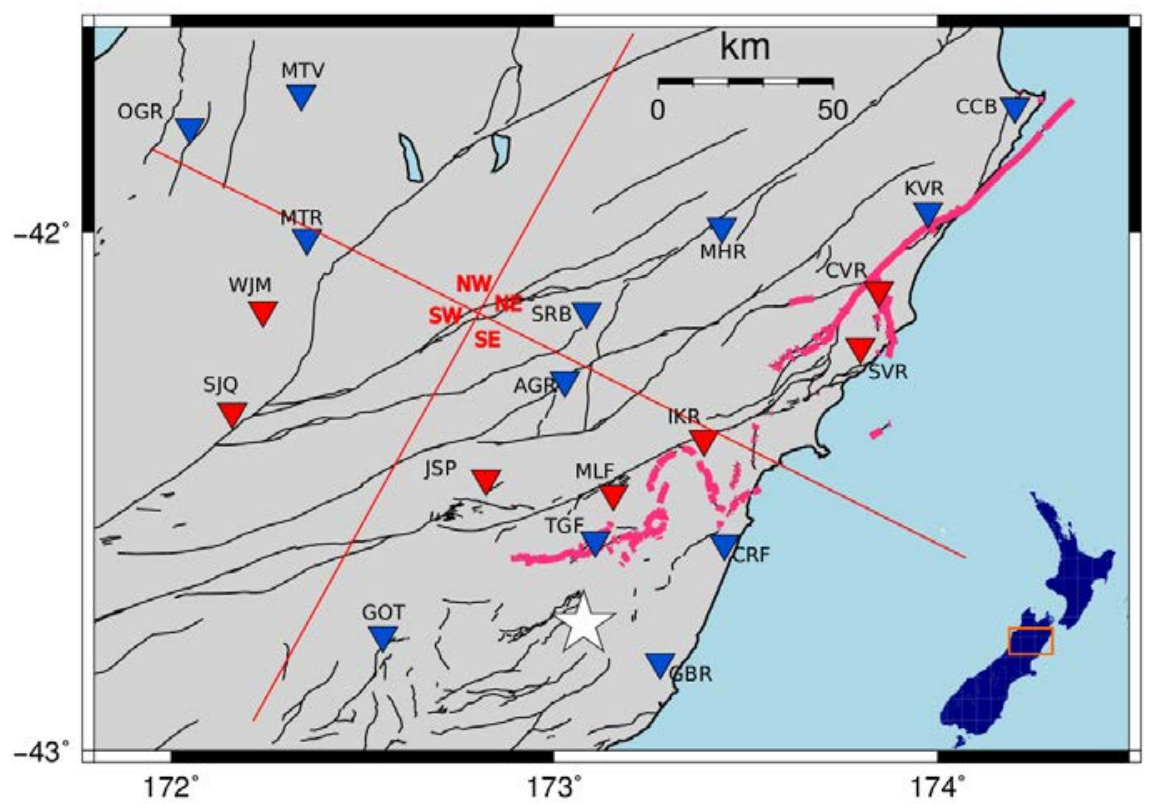

Figure 6.3: Station map with the region divided into four different quadrants to be analysed. Red triangles are stations that are being used and blue triangles are stations that are not being used. The white star indicates the location of the Kaikôura earthquake. The pink lines are the faults that ruptured in the Kaikōura earthquake with the black lines showing all other faults in the region. The red lines split the region into four separate quadrants (NE, SE, SW, and NW).

This analysis was split between station pairs within each quadrant and station pairs across each quadrant. For this analysis, stations CVR, IKR, JSP, MLF, SJQ, SVR and WJM are used as shown in Figure 6.3. These stations were chosen as they produced some of the clearest ZZ component results and are also spread around the region. Firstly, we analyse pairs within each quadrant to determine small interstation distances. Within each quadrant the best stations pairs were chosen. These pairs produced the most coherent waveforms and had the longest continuous duration.

In Figure 6.4, the ZZ component of three different station pairs (CVR-SVR, IKRMLF, and SJQ-WJM) are plotted. The first, CVR-SVR, is in the north-eastern quadrant. This was the only pair in this quadrant that produced a usable result, as a few other station pairs had large time gaps. This station pair was not recording for the full duration, but it shows a large velocity decrease (larger than the other two pairs at approximately $1.2 \%$ ) following the Kaikōura earthquake. Possibly this 


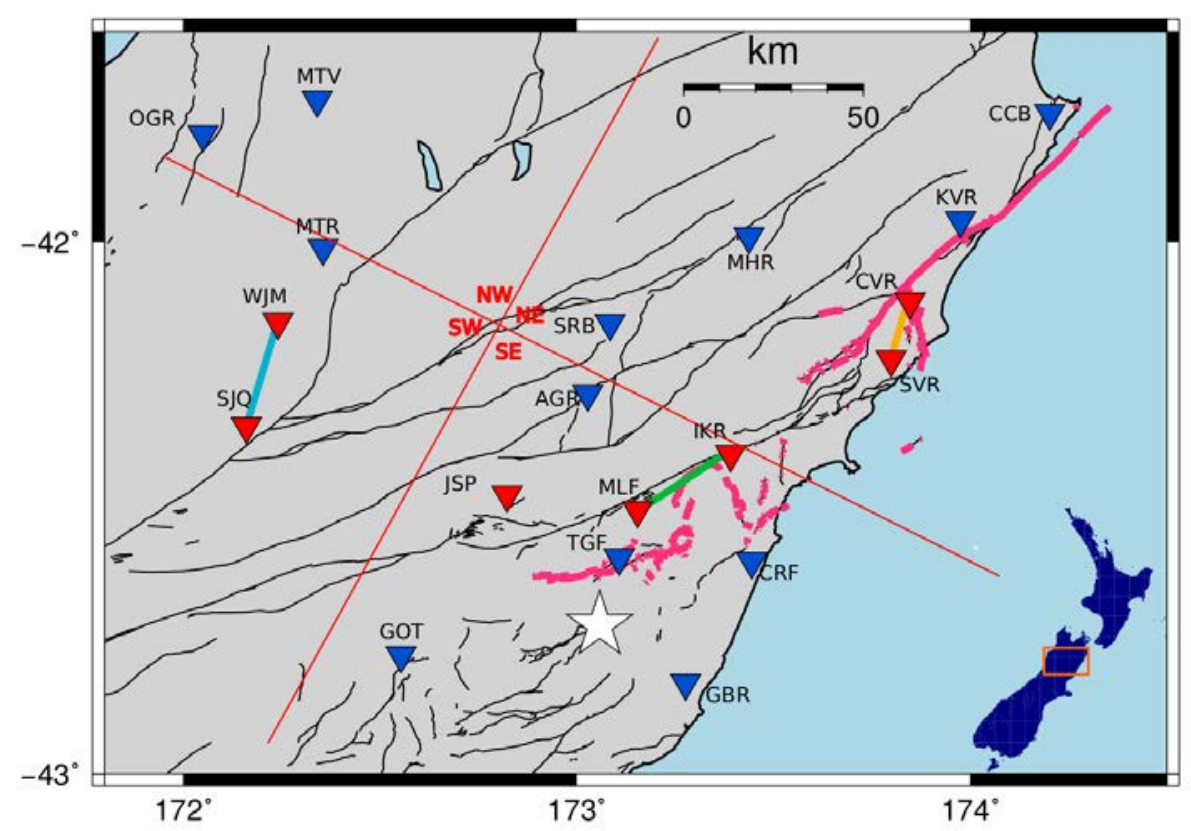

(a) Map of location of station pairs.

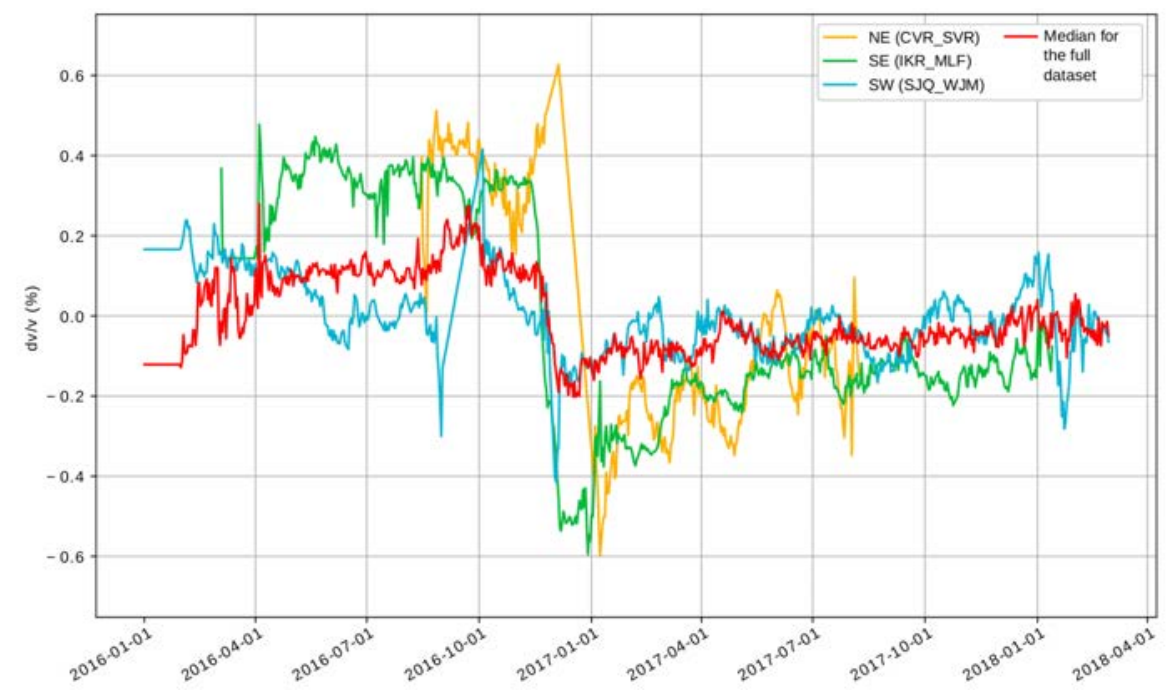

(b) Velocity changes for station pairs shown in the map in Figure 6.4a.

Figure 6.4: Velocity variation for station pairs within each quadrant. a) Station map with region divided into four different quadrants to be analysed where station pairs are coloured the same as in Figure 6.4b. Red triangles are stations that are being used and blue triangles are stations that are not being used. The white star indicates the location of the 2016 Kaikōura earthquake. The pink lines are the faults that were ruptured in the 2016 Kaikōura earthquake with the black lines showing all other faults in the region. The red lines split the region into four separate quadrants (NE, SE, SW, and NW). b) Velocity changes for station pairs using a 30-day moving window for the vertical-vertical component for a two-year duration using a two-year reference period. 
decrease was much larger because the path between CVR and SVR crosses quite a few ruptured faults and CVR is almost directly on a fault (Refer to Section 2.2 in Background Theory and Section 6.2.4). The velocity decrease for the Kaikoura earthquake for CVR-SVR also appears to be delayed. This could be a result of the station pair having poor data quality within this month, so the moving average values are delayed and the first and last values of this average are exaggerated. Another station pair to produce quite a large velocity decrease (approximately $1.0 \%$ ) was station pair IKR-MLF. Station pair IKR-MLF is in the south-eastern quadrant and, like CVR-SVR, also has an interstation path that crosses several ruptured faults. A station pair in the south-western quadrant, SJQ-WJM, has the smallest velocity decrease of the three pairs at approximately $0.2 \%$. However, this value is the most consistent (out of the three pairs in Figure 6.4) with the overall average in the region. This velocity decrease is also much more gradual than the other station pairs and this is most likely a factor of SJQ-WJM being the furthest away from the ruptured faults and the location of the Kaikōura earthquake. Although these values vary greatly between different station pairs, the station pairs which have an interstation path that crosses multiple faults seem to have had the greatest changes in velocity following the Kaikōura earthquake.

Secondly, station pairs can be analysed across different quadrants (Figure 6.5). This is shown in a similar way as the station pairs within each section was shown in Figure 6.4 .

The results for different station pairs across different quadrants (Figure 6.5) are more similar to each other than to the station pairs within each quadrant. All of the station pairs show a velocity decrease following the 2016 Kaikōura earthquake, except for station pair JSP-SJQ. This pair is quite erratic and does not seem to follow a similar trend to the other station pairs. This is possibly due to JSP-SJQ not crossing any of the ruptured faults (as shown by the pink lines in Figure 6.5a). A few other station pairs also follow a similar direction (east-west) and do not cross many ruptured faults as JSP-SJQ does; these being IKR-SJQ, MLF-WJM, SJQ-SVR, and 


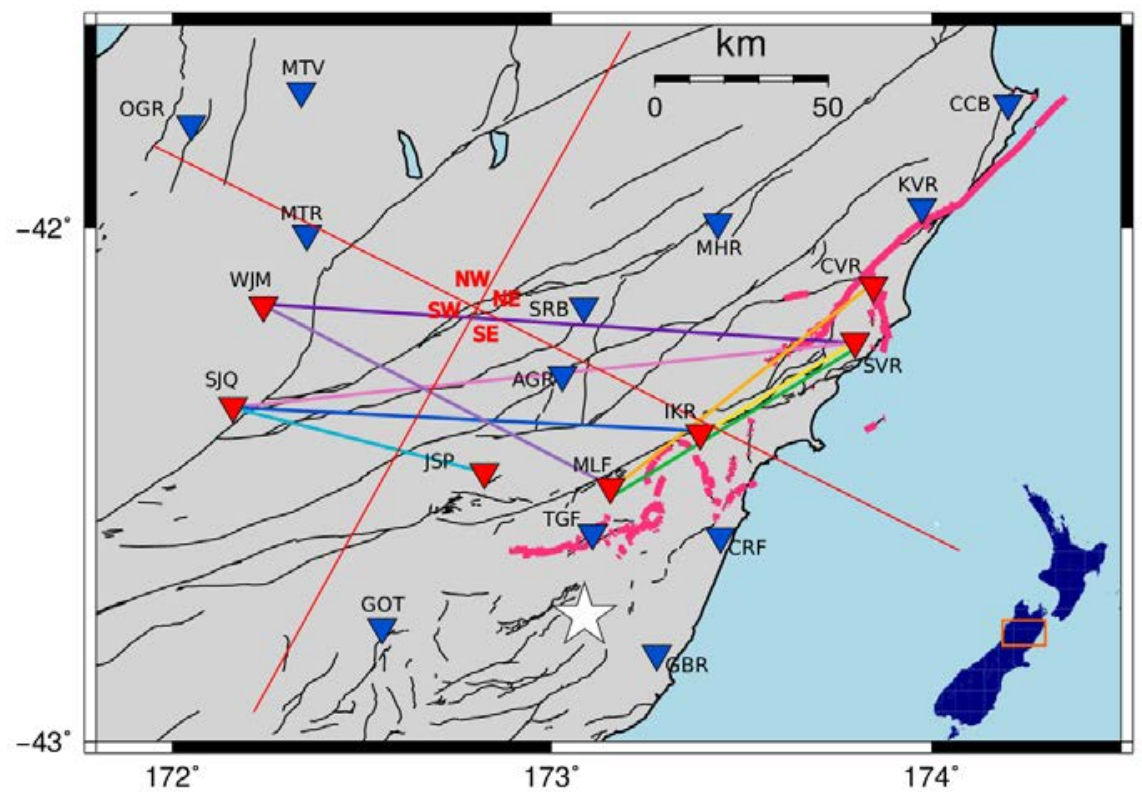

(a) Map of location of station pairs.

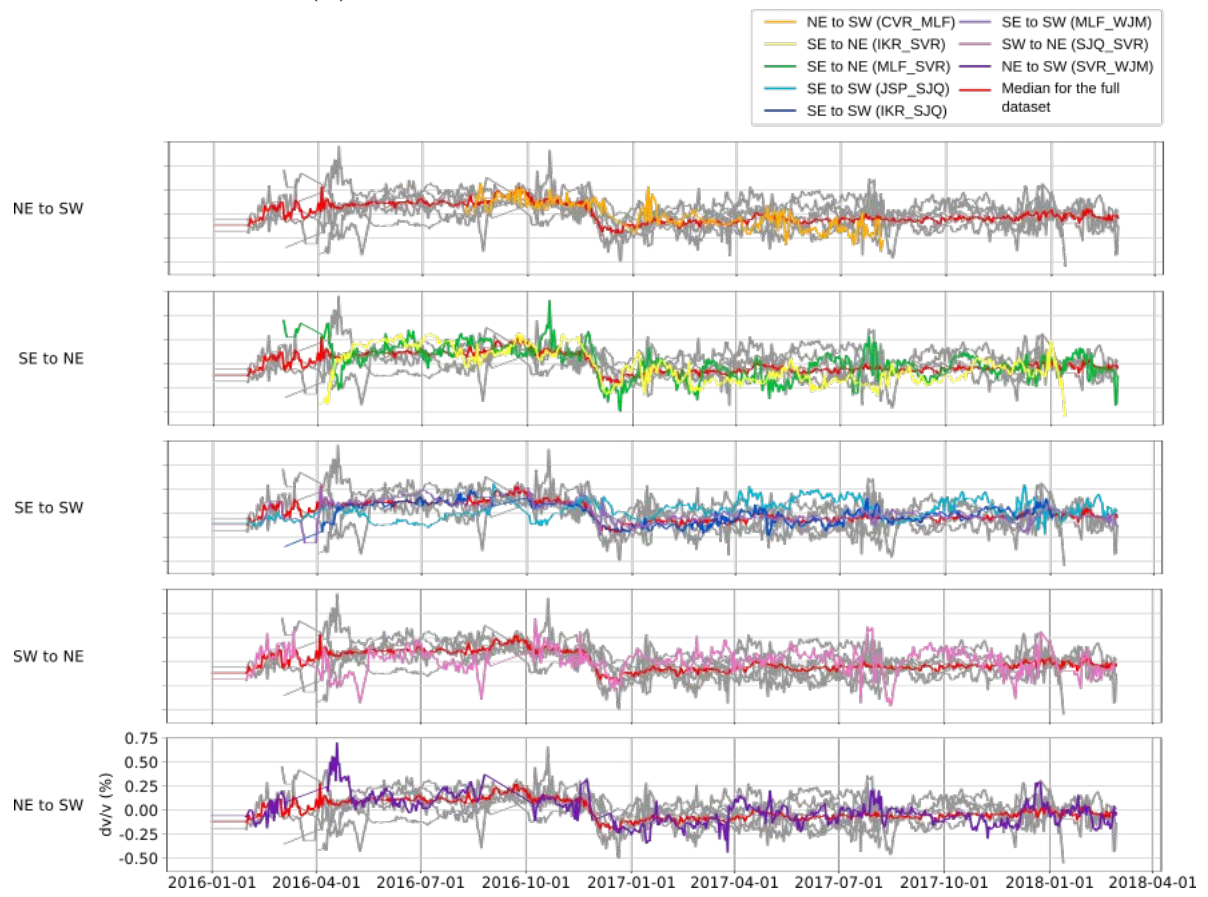

(b) Velocity changes for station pairs shown in the map in Figure 6.5a.

Figure 6.5: Velocity variation for station pairs across each quadrant. a) Station map with region divided into four different quadrants to be analysed where station pairs are coloured the same as in Figure 6.5b. Red triangles are stations that are being used and blue triangles are stations that are not being used. The white star indicates the location of the 2016 Kaikōura earthquake. The pink lines are the faults that were ruptured in the 2016 Kaikōura earthquake with the black lines showing all other faults in the region. The red lines split the region into four separate quadrants (NE, SE, SW, and NW). b) Velocity changes for station pairs using a 30-day moving window for the vertical-vertical component for a two-year duration using a two-year reference period. Plots split by direction of station pair paths, median result for full dataset shown in each plot in red. Axes values for the first four plots are the same as the bottom plot. 
SVR-WJM. This is also observed in Figure 6.4 for station pair SJQ-WJM. These station pairs, unlike JSP-SJQ, (Figure 6.5) do seem to show a velocity decrease following the Kaikōura earthquake. This is most likely due to the fact that IKR, MLF, and SVR are all located in quite close proximity $(\leq 10 \mathrm{~km})$ to ruptured faults. So even if one of the stations (SJQ and WJM) wasn't in an area with an overall large change, the other station was. However, station pair SJQ-WJM contradicts this as despite SJQ and WJM being far away, the station pair SJQ-WJM does observe a decrease following the Kaikōura earthquake (Figure 6.4).

From Figures 6.4 and 6.5, station pairs that we would expect a strong result from are those that crossed multiple ruptured faults, such as CVR-MLF, IKR-SVR, and MLF-SVR. These three pairs all show a clear velocity decrease following the 2016 Kaikōura earthquake. As shown by JSP-SJQ, as we move further away from the Kaikōura earthquake location and the ruptured faults $(>50 \mathrm{~km})$, less of a decrease in velocity is visible. Another pair that is far away is SJQ-WJM (>100 km) and it still shows a decrease following the Kaikōura earthquake (Figure 6.4). However, it does also show a large velocity decrease before increasing rapidly before the earthquake, which other pairs do not show. Furthermore the decrease following the earthquake from this pair is more gradual than pairs closer to ruptured faults, so it is possible that it is a delayed result and the reason JSP-SJQ shows a poor result is merely due to poor station quality. As all station pairs show a different result due to location and station quality, this reinforces the idea that the most accurate result for the region is obtained by averaging all of the station pairs. This reduces erratic behaviour from single station pairs.

\subsection{Velocity Changes}

The Kaikōura region (area shown within Figure 6.6) has experienced 31 earthquakes from the $1^{\text {st }}$ of January 2012 to the $28^{\text {th }}$ of February 2018 greater than magnitude 5.5 (earthquake data acquired from GeoNet). Nine of these earthquakes are greater than 
magnitude six, with six of these earthquakes (including the main event $-M_{w} 7.8$ ) occurring on the $14^{\text {th }}$ of November 2016. Earthquakes with $M_{w}>5.5$ are shown in Figure 6.6. In this figure, earthquakes with a magnitude greater than six are labelled with a number and referenced in Table 6.1. Also mentioned in this table are three earthquakes that did not occur on the day of the 2016 Kaikoura earthquake or in the Cook Strait earthquake sequence that have a magnitude in the order of $M_{w} 5.5-6$.

\begin{tabular}{|c|c|c|c|}
\hline Number on Map & Date (NZDT) & Magnitude & Depth $(\mathrm{km})$ \\
\hline 1 & $2013-07-21$ & 6.5 & 15.6 \\
\hline 2 & $2013-08-16$ & 6.6 & 7.5 \\
\hline 3 & $2015-04-24$ & 6.2 & 51.5 \\
\hline 4 & $2016-11-1400: 02$ & 7.8 & 15.1 \\
\hline 5 & $2016-11-1400: 05$ & 6.0 & 4.8 \\
\hline 6 & $2016-11-1400: 12$ & 6.0 & 5.6 \\
\hline 7 & $2016-11-1400: 32$ & 6.2 & 11.3 \\
\hline 8 & $2016-11-1400: 52$ & 6.1 & 9.0 \\
\hline 9 & $2016-11-1413: 34$ & 6.7 & 5.0 \\
\hline 10 & $2016-02-09$ & 5.7 & 48.1 \\
\hline 11 & $2016-11-22$ & 5.8 & 7.2 \\
\hline 12 & $2017-10-22$ & 5.6 & 8.3 \\
\hline
\end{tabular}

Table 6.1: Table describing the numbers annotating earthquakes in Figure 6.6. A time (NZDT) is added to the date if multiple earthquakes occur on the same day (4-9). All of the earthquake information is from GeoNet.

Three different time periods of data in the Kaikôura region are analysed to see if there were any changes in velocity using a 30-day, 60-day, and 90-day moving window. The mean and median result from these $N$-day windows are shown in Figure 6.7. These results are plotted to show that there is not much difference between the mean and the median values for these three $N$-day moving windows. As a result of this, only the median velocity change results will be plotted when analysing the three different time periods. The 90-day moving window also does not show a large velocity decrease at the beginning of the time series. This shows that this velocity decrease occurs due to the first few days in both the 30-day and the 60-day moving average (Figure 6.7). As the 60-day and 90-day moving window have quite a large delay and show a similar decrease to the 30-day moving window, the 30-day window will be used to more closely examine specific time periods. The time periods of interest to 


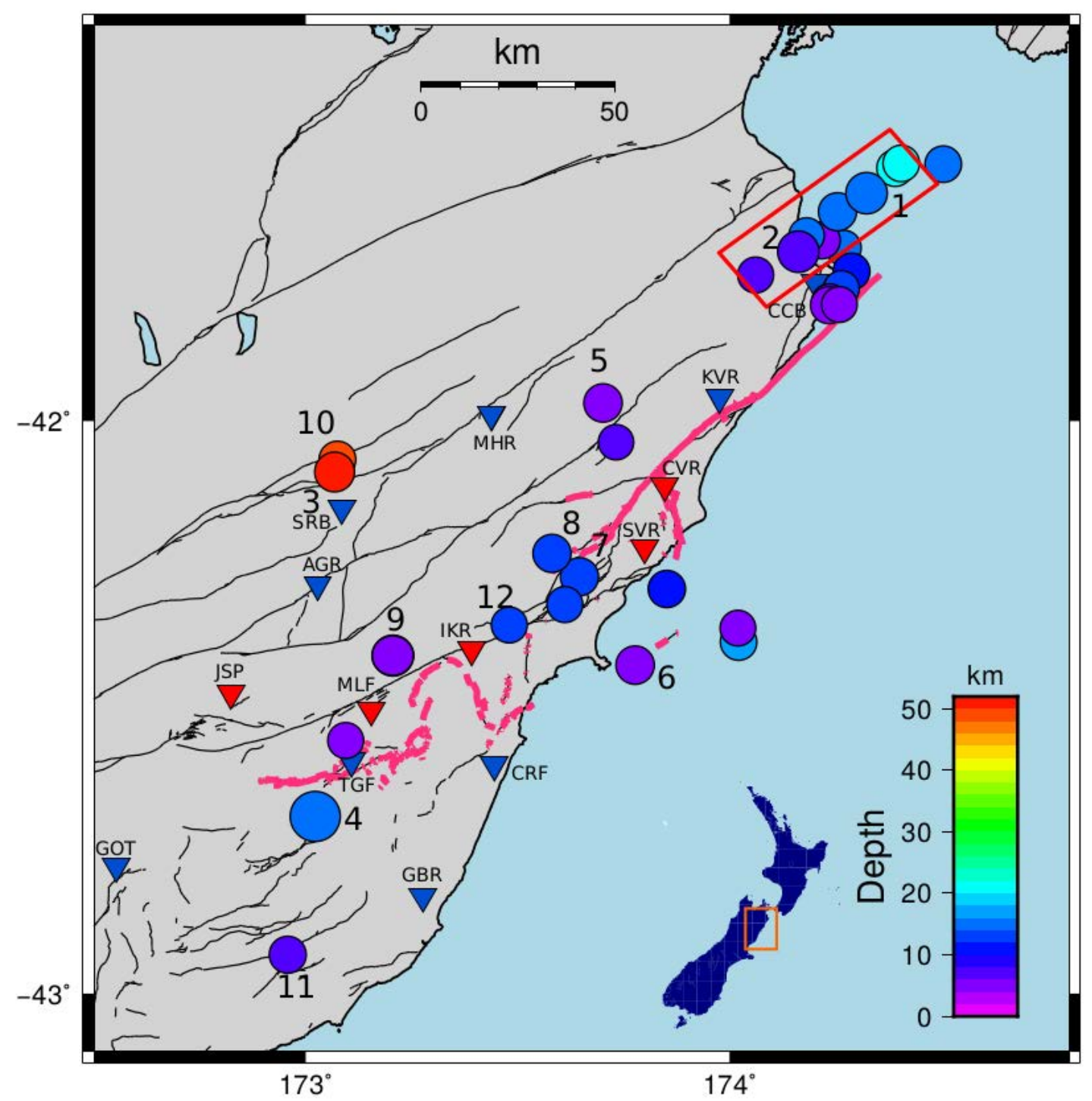

Figure 6.6: Map with earthquake locations greater than Magnitude 5.5 from 201201-01 to 2018-02-28. The numbered earthquakes correspond to those listed in Table 6.1. The first nine numbers are for earthquakes with $M_{w}>6.0$. The remaining 3 numbers (10-12) are for earthquakes between $M_{w} 5.5-6.0$ of interest. The red box marks the 2013 Cook Strait earthquake sequence. Black lines denote the faults, with pink lines showing the faults that ruptured during the 2016 Kaikoura earthquake. Red triangles show the location of the stations that are used, blue triangles are unused stations. Key earthquakes in this map are: 1=Cook Strait 2013, 2=Lake Grassmere 2013, 3=April 2015, and 4=Kaikōura 2016.

be discussed are July-August 2013 (Section 6.2.1), April 2015 (Section 6.2.2), and November 2016 (Section 6.2.3).

Seven large $\left(M_{w}>5.6\right)$ earthquakes have been plotted on the median moving windows for the same $N$-day averages in Figure 6.8 as shown in Figure 6.7. 


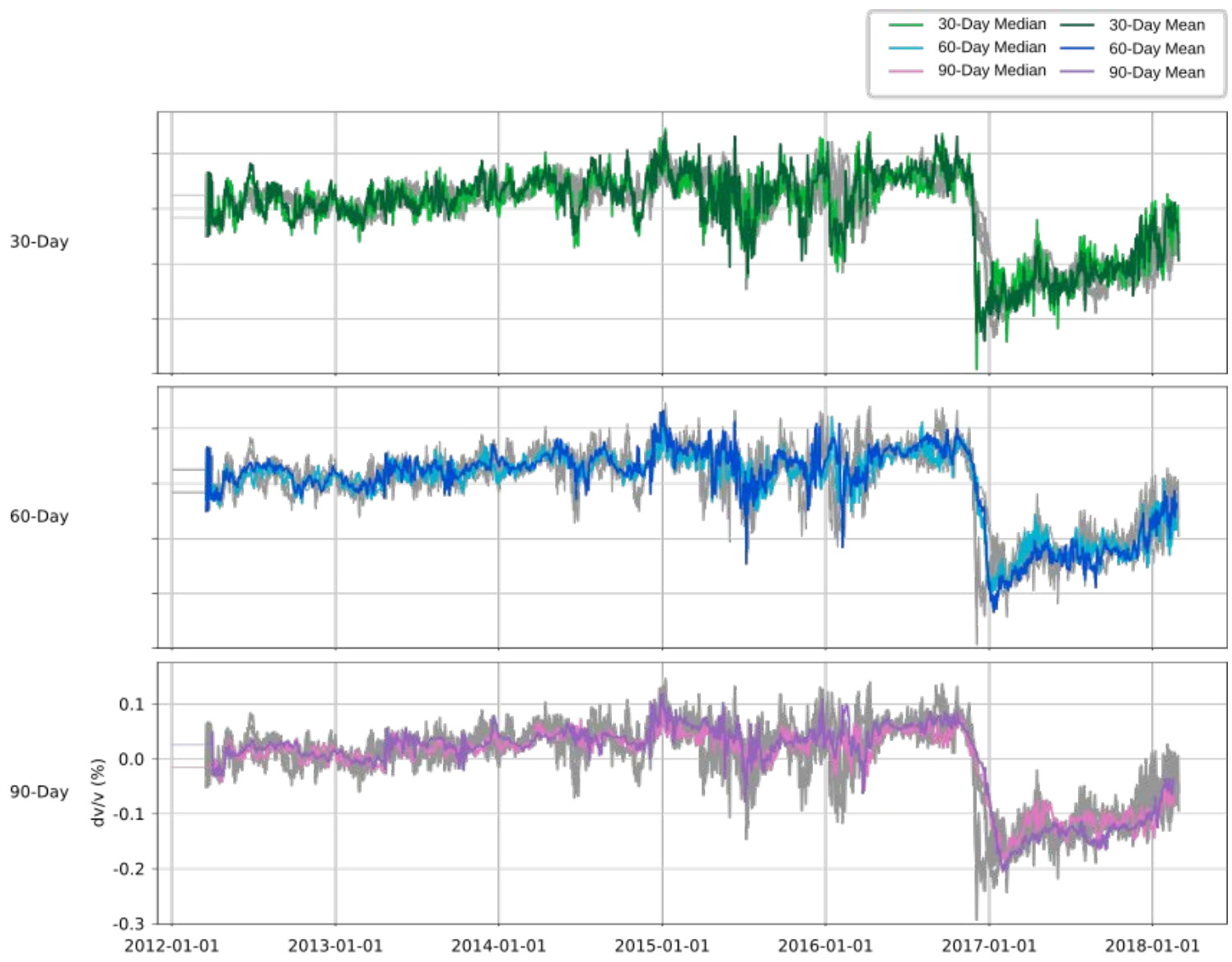

Figure 6.7: Velocity variation plot of median and mean velocity changes for 30-day, 60-day and 90-day moving windows. These windows are the result of the velocity on a given day as the average of the previous $N$-days including itself. A seven-year reference period was used for this seven-year dataset. Axes values for the top two plots are the same as the bottom plot.

\subsubsection{Cook Strait earthquake sequence 2013}

During 2013, there were two large magnitude events: the Cook Strait earthquake ( $M_{w} 6.5$ in July 2013), and the Lake Grassmere earthquake $\left(M_{w} 6.6\right.$ in August 2013). Both of these earthquakes were located quite close to one another (17.3 km apart) and quite shallow at 15.6 and $7.5 \mathrm{~km}$ deep respectively. In the two day's prior to the Cook Strait earthquake there was also a $M_{w} 5.7$ and a $M_{w} 5.8$. The $M_{w}>6.0$ events are labelled (in Figure 6.6) and the two events prior to the Cook Strait earthquake are plotted as part of the Cook Strait earthquake sequence in the red box in Figure 6.6 .

Despite this being such a large event, no significant velocity changes are seen in Figure 6.8 as a result of it. This is possibly because not many stations with usable 


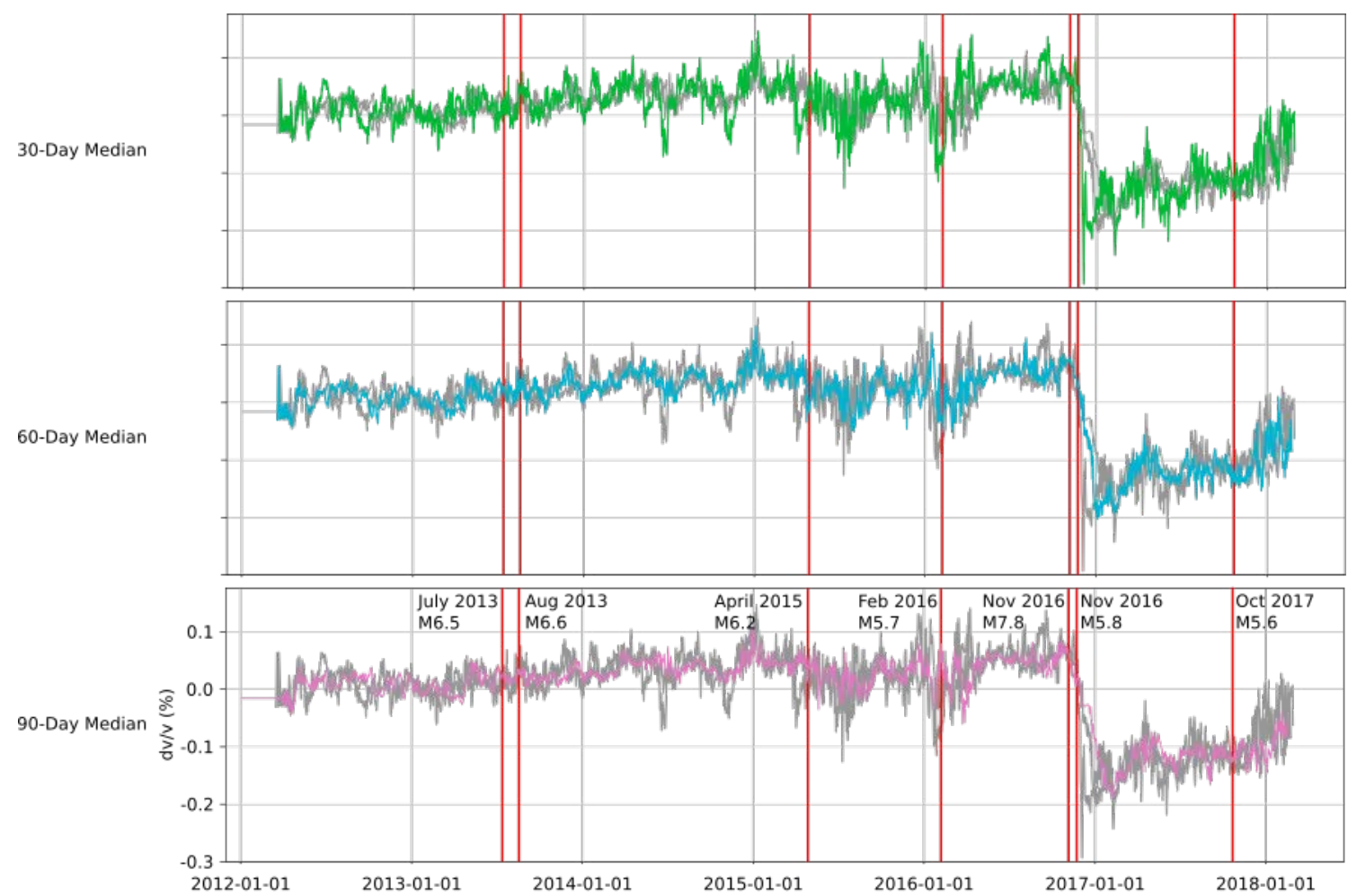

Figure 6.8: Velocity variation plot of median velocity changes for 30-day, 60-day and 90-day moving windows. These windows are the result of the velocity on a given day as the average of the previous $N$-days including itself. A seven-year reference period was used for the seven-year dataset. The red lines indicate earthquake events with magnitudes greater than 5.6. More details on each earthquake can be found in Table 6.1. Axes values for the top two plots are the same as the bottom plot.

data are close to the area that ruptured during the Cook Strait and the Lake Grassmere earthquake. The closest station to the Lake Grassmere and the Cook Strait earthquake is CCB (see Figure 6.6) with station KVR also close. Despite this close proximity, neither station produced usable velocity change results, as CCB had low correlation coefficient values and KVR did not have any data during this period (Figure 3.2). As a result of this, the closest pair is CVR-SVR, which is approximately $60 \mathrm{~km}$ away (from the location of the Lake Grassmere earthquake to station CVR). Also, station SVR had only been deployed a few months prior (May 2013) and when analysing data from the station pair CVR-SVR it does not appear reliable. This is a result of gaps in the data and larger fluctuations in velocity in the following years than was observed following the Cook Strait and Lake Grassmere earthquakes (Figure 6.9). The next closest station to the Cook Strait earthquake sequence is IKR (see Figure 6.6). Station IKR was deployed in April 2013 and due to errors in data 
collection/duration, velocity changes are only observed after 2014 for station pairs CVR-IKR and IKR-SVR (Figure 6.9).

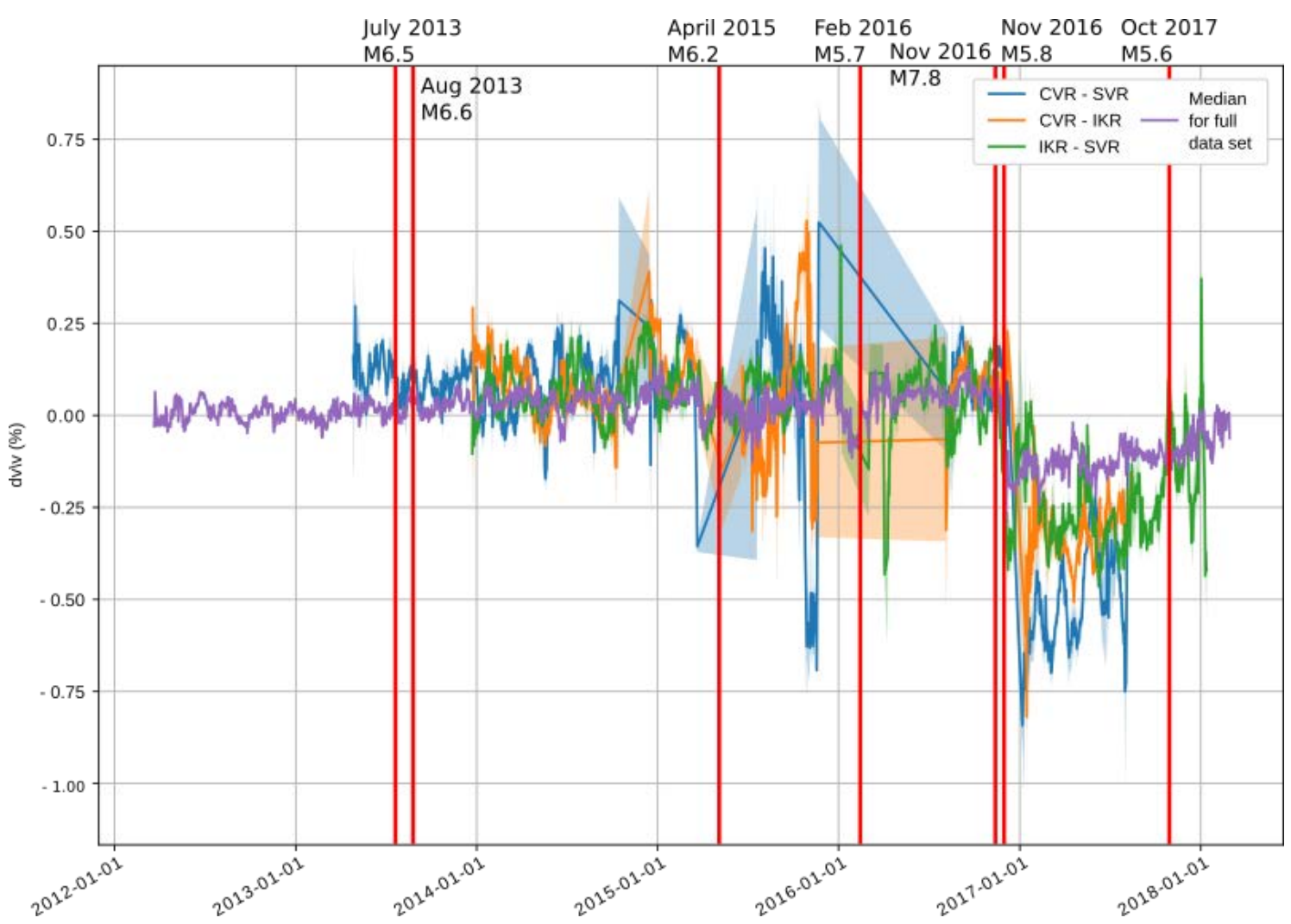

Figure 6.9: Velocity variation plot of median velocity changes for a 30-day moving window for different station pairs close to the 2013 Cook Strait and Lake Grassmere earthquakes. Red lines show the Cook Strait and Lake Grassmere earthquakes as well as other large magnitude earthquakes $\left(M_{w}>5.6\right)$. All three of these station pairs are missing data at a few stages, shown by the solid lines and the transparent blocks showing increased error in these time periods. These sections also have a few data points on either side that would be slightly exaggerated as the moving average would not be properly calculated.

Due to the lack of data, we conclude that the DPRI stations did not record a change in velocity as a result of the Cook Strait earthquake sequence, or the Lake Grassmere earthquake. This is not to say that a velocity change did not occur, merely that the DPRI stations potentially were not close enough or maybe the DPRI stations had too much noise to observe these small changes. Civilini et al. (2020) found a velocity decrease of $0.06 \%$ after each of the earthquakes at Ngatamariki Geothermal Field, and the stations he used were located in the Taupō Volcanic Zone, which is just under $400 \mathrm{~km}$ away, much further than our stations to the Cook Strait. Brenguier et al. (2014) found that following the $2011 M_{w}$ 9.0 Tohoku-Oki earthquake the strongest 
coseismic velocity reductions occurred under volcanic regions rather than regions with the most intense ground shaking or the coseismic deformation. They suggest that regions with low effective pressure, such as volcanic or geothermal regions are more susceptible to velocity changes and dynamic stress changes. The opening of cracks and increasing the permeability and transfer of fluids is discussed as the mechanism by which seismic velocities decrease (Brenguier et al., 2014). Under volcanic areas, the effective pressure in the crust can be reduced because of the presence of highly pressurized hydrothermal and volcanic magmatic fluids at depth. As the sensitivity of the seismic velocity to stress changes in the rock increases with decreasing pressure, seismic velocity changes are more easily detected in volcanic areas. This is potentially why, Civilini et al. (2020) observed velocity changes from the Cook Strait earthquake sequence in a geothermal region, which would have highly pressurized hydrothermal fluids at depth, and we did not.

\subsubsection{Earthquake in April 2015 and February 2016}

Another large earthquake occurred in April 2015 and was a $M_{w} 6.2$ (number three in Figure 6.6). This earthquake was quite deep at $52 \mathrm{~km}$. There was also another earthquake approximately $5 \mathrm{~km}$ away in February 2016 with a magnitude of 5.7 which was also quite deep at $48 \mathrm{~km}$ (number ten in Figure 6.6). Velocity change results from these two earthquakes could be the reason for the velocity decreases of approximately $0.1 \pm 0.04 \%$ (for both events) slightly after both events in the middle of 2015 and at the beginning of 2016 (see Figure 6.8). The event in April 2015 created a slightly larger velocity decrease than in February 2016 and this is possibly due to the 2015 event having a slightly larger magnitude. The closest station pair to these events is JSP-SRB. However, unfortunately, SRB stopped recording after February 2015, resulting in this pair not having any data related to either of these two events. Looking slightly further afield, stations IKR, MLF, and JSP may contain useful information. Station pair IKR-JSP, shows a slight velocity increase and decrease of approximately $0.4 \%$ whereas IKR-MLF is missing data from January 2015 until 
February 2016 (see Figure 6.10). Station pair CVR-WJM was also checked as this ray path crosses the location of the earthquake, however as this pair is missing data, it is not useful.

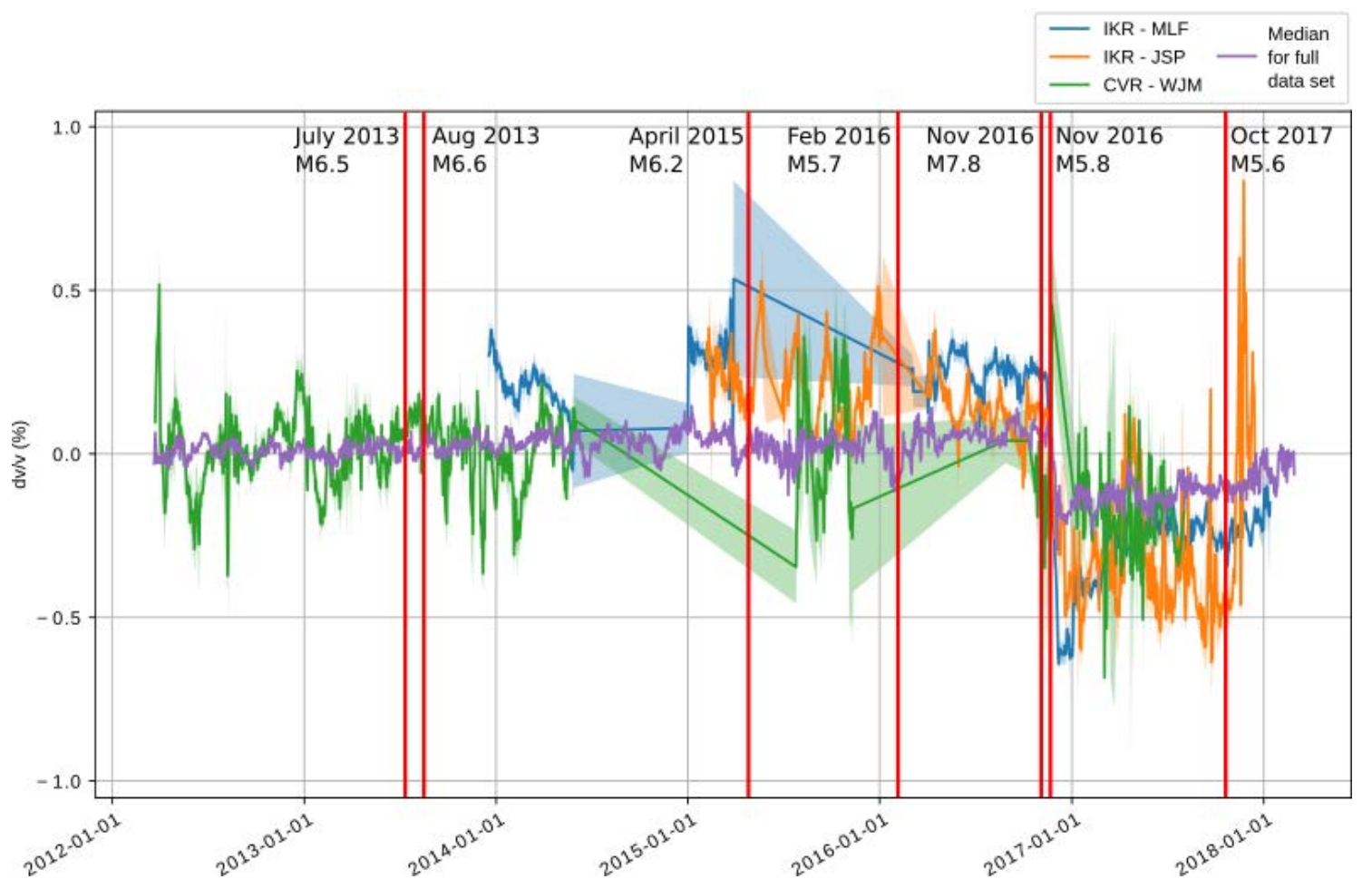

Figure 6.10: Velocity variation plot of median velocity changes for a 30-day moving window for different station pairs close to the April 2015 and the February 2016 earthquakes. Red lines show the April 2015 and the February 2016 earthquakes as well as other large magnitude earthquakes $\left(M_{w}>5.6\right)$. All three of these station pairs are missing data at a few stages, shown by the solid lines and the transparent blocks showing increased error in these time periods. These sections also have a few data points on either side that would be slightly exaggerated as the moving average would not be properly calculated.

Velocity changes for the two large magnitude events that occurred in April 2015 and February 2016 can be observed in nearly all station pairs and in the overall result shown in Figure 6.8. However, the velocity changes are very small $(\sim 0.1 \%)$ because quite a few stations close to these earthquakes were not recording during this period of time and the two earthquakes are both quite deep (52 km and $48 \mathrm{~km})$. As the April 2015 earthquake was quite deep at $52 \mathrm{~km}$ depth, it is likely that the gradual velocity decrease and following increase is a result of stations being far away from the source. A gradual increase and decrease in velocity similar to this was observed by SJQ-WJM following the 2016 Kaikōura earthquake (Figure 6.4). 


\subsubsection{The 2016 Kaikōura earthquake}

The surface wave velocity decrease of $0.24 \pm 0.02 \%$ following the 2016 Kaikōura earthquake is comparable to that observed in other similar studies elsewhere. A $0.14 \pm 0.04 \%$ increase in velocity was recorded in the two-and-a-half-months following the lower magnitude $M_{w} 7.12010$ Darfield earthquake, and attributed to the recovery of velocity after an unknown amount of decrease at the time of the earthquake (Heckels, 2017). A large velocity decrease of $1.5 \%$ was shown during a six-month period centered around the $2011 M_{w} 9.0$ Tohoku-oki earthquake (Minato et al., 2012). As in this study and with Heckels (2017) and Minato et al. (2012), a gradual healing process was displayed after the earthquakes involved. In this study, the velocity change has not quite fully recovered to pre-earthquake values of $0.5 \pm 0.02 \%$ by February 2018 (see Figure 6.8). There is also a gradual increase observed before the Kaikōura earthquake as a result of using a reference period for the full duration (see Figure 5.11).

\begin{tabular}{|c|c|}
\hline Component & Velocity Decrease (\%) \\
\hline EE & $0.2 \pm 0.02$ \\
\hline EN & $0.22 \pm 0.04$ \\
\hline EZ & $0.19 \pm 0.03$ \\
\hline NE & $0.32 \pm 0.1$ \\
\hline NN & $0.24 \pm 0.04$ \\
\hline NZ & $0.21 \pm 0.1$ \\
\hline ZE & $0.16 \pm 0.04$ \\
\hline ZN & $0.19 \pm 0.02$ \\
\hline ZZ & $0.24 \pm 0.02$ \\
\hline
\end{tabular}

Table 6.2: Relative velocity decreases by cross-correlation component for all stations averaged from the beginning of the decrease to the end of the decrease. Variability is shown between cross-components by the error bars as determined by the start and end point of each decrease. This is often clearer in some cross-components than in others (for example, it was easier to see the decrease in EN than in NE).

A velocity decrease following the 2016 Kaikōura earthquake was observed across the majority of station pairs and over all cross-components. This velocity decrease for each cross-component is listed in Table 6.2. The strongest result was observed on the vertical-vertical component with a velocity decrease of $0.24 \pm 0.02 \%$. Only one station pair did not show a velocity decrease following the earthquake and this was JSP-SJQ 
(see Figure 6.5). This is most likely a result of this station pair not intersecting with any of the ruptured faults and specific problems with these individual stations because station pair SJQ-WJM which was quite far from the ruptured faults and still showed a velocity decrease following the Kaikōura earthquake (see Figure 6.4). The velocity change shown by station pair SJQ-WJM decreases more slowly than station pairs that are closer to ruptured faults (see station pair IKR-MLF in Figure 6.4 for example). This suggests that the velocity change is affected by distance between the source location and the stations. Interstation distance will also be a factor, as stations with longer interstation distances and further away from ruptured faults (MLF-WJM for example in Figure 6.5) will have a smaller decrease in velocity than pairs with a smaller interstation distance and closer to the ruptured faults (for example IKR-MLF in Figure 6.4).

\subsubsection{Mechanisms for velocity changes}

Several possible mechanisms for velocity changes are discussed in the Background Theory chapter (Section 2.2). These possible mechanisms are 1) stress-induced changes of fault zone properties, 2) damage of the crust from fault zone rupture and subsequent healing, 3) damage of the crust from strong-ground shaking, and 4) rapid changes in groundwater near the surface or fluid activities in the shallow crust.

Stress-induced velocity changes can be both positive and negative and relate to regions with increased and decreased stress. These changes are often deep and are caused by cracks opening and closing. This is possibly a contributing mechanism for the 2016 Kaikōura earthquake velocity changes. However, as most of the changes observed were shallow, between $2.5-5 \mathrm{~km}$ deep (Figure 6.2 ), it is unlikely that the changes were entirely related to deep stress-induced movements.

Damage of the crust from the fault zone rupture and the subsequent healing results in first a velocity decrease and then a velocity increase as crack healing occurs. As many as 21 faults (Stirling et al., 2017) were ruptured as a result of the 2016 Kaikoura 
earthquake and as such the entire region was effected. This is evidenced in velocity decreases across all station pairs, even the ones which had paths that did not cross ruptured faults (see Figures 6.4 and 6.5). These same station pairs that did not cross ruptured faults also then showed a gradual velocity increase as cracks healed following the earthquake. This is determined to be the strongest contributing mechanism as these changes were observed over the entire region, as would be expected when cracks occur in the fault zone.

Another likely mechanism is through damage of the shallow crust from strong ground shaking. This is likely as the frequencies observed, $0.1-0.9 \mathrm{~Hz}$, equate to shallow crustal depths of the upper 2.5-5 km. Following the 2016 Kaikoura earthquake, peak ground velocities $(\mathrm{PGV}$ ) reached $80 \mathrm{~cm} / \mathrm{s}$ as shown in Figure 6.11a by Bradley et al. (2017). 47, 16, and 5 ground motions were observed across the region with peak ground acceleration, PGA $>0.1 \mathrm{~g}, 0.2 \mathrm{~g}$, and $0.5 \mathrm{~g}$ respectively (Bradley et al., 2017). Wu et al. (2003) demonstrate through a regression analysis that earthquake damage correlates much better with PGV values than with PGA values. As such, PGV values will be compared to illustrate the effects of ground shaking. Figure 6.11 demonstrates how different velocity pairs were affected by different amounts of ground shaking following the Kaikōura earthquake. These station pairs were chosen to demonstrate changes with two pairs in a high region of shaking, $80 \mathrm{~cm} / \mathrm{s}$ (IKR-MLF and IKR-SVR), a station pair with one station in a high region of shaking and the other in a lower region of shaking (IKR-SJQ), and a station pair in a region with the least amount of shaking, $10 \mathrm{~cm} / \mathrm{s}(\mathrm{SJQ}-\mathrm{WJM})$. Pairs within the region with the strongest shaking experienced the largest decrease, with IKR-MLF having a decrease of $\sim 1.0 \%$ (also shown in Figure 6.4). SJQ-WJM shows the least amount of shaking and records a more gradual decrease following the earthquake. Station pair IKR-SJQ, experienced strong and weak shaking at each station respectively and also shows a velocity decrease similar to the median result. These results suggest that larger velocity decreases can be correlated to station pairs that experienced stronger ground motions. Therefore, strong ground motions caused damage may be one of the mechanisms explaining the velocity changes. 


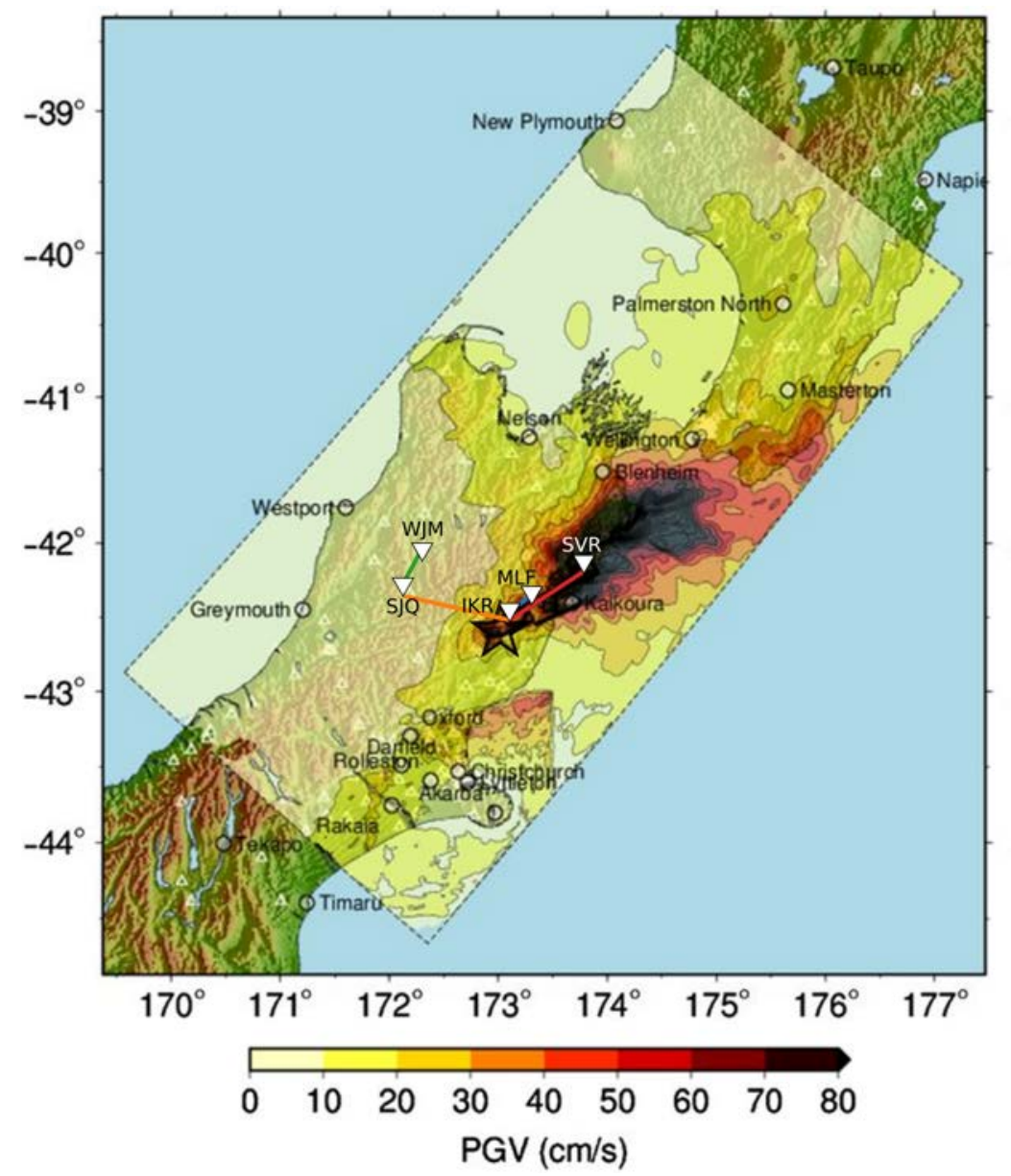

(a) Peak Ground Velocity following the 2016 Kaikōura earthquake (Bradley et al., 2017).

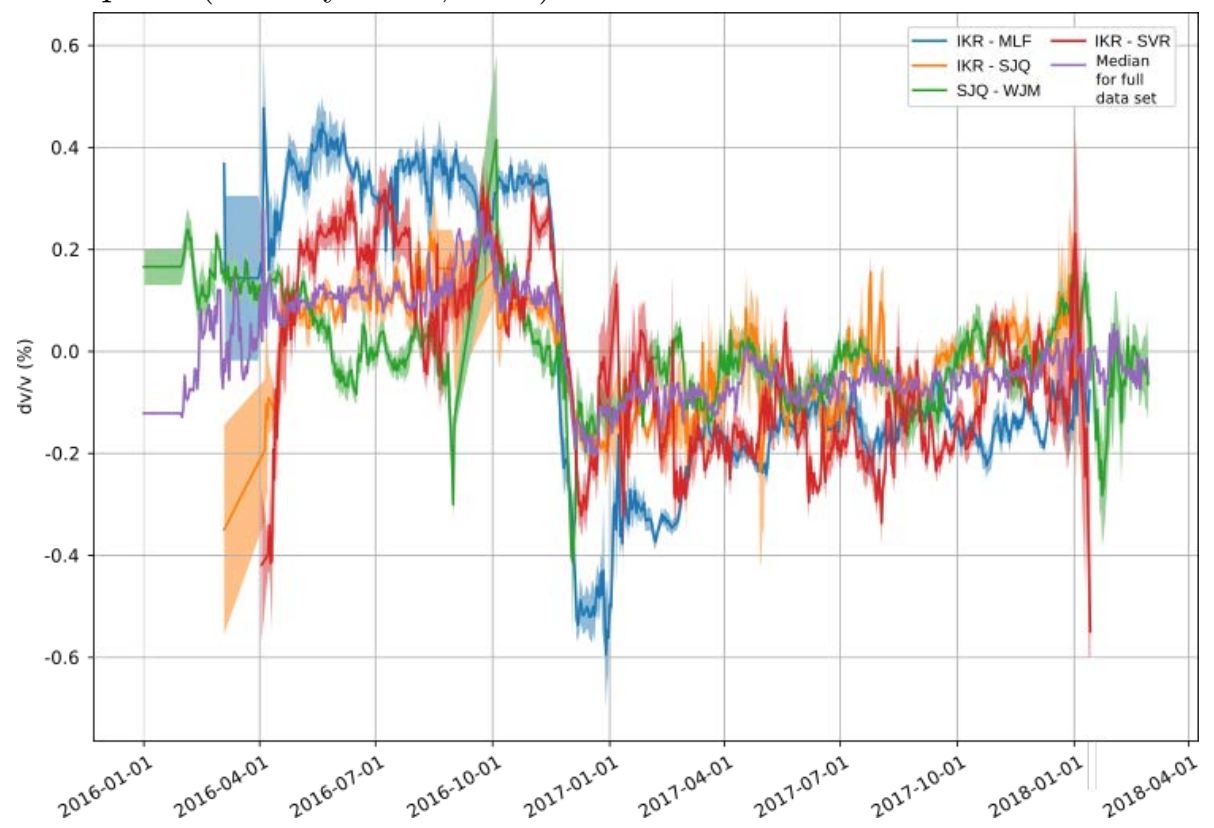

(b) Velocity changes for station pairs shown in the map in Figure 6.11a.

Figure 6.11: Velocity variation for station pairs across varying peak ground velocity (PGV) values. a) PGV from the ground motion simulation performed by Bradley et al. (2017). Coloured station pair paths correlate to station pair velocity variation results in b). b) Velocity changes for station pairs using a 30-day moving window for the vertical-vertical component for a two-year duration using a two-year reference period. 
Short-term groundwater responses and other fluid movements can cause changes in seismic velocities. Weaver et al. (2019) observed earthquake induced water level changes at distances between 4 and $850 \mathrm{~km}$ from the 2016 Kaikōura earthquake epicentre. These water level changes were inconsistent with static stress changes but did correlate with peak ground acceleration. The time taken for water levels to reequilibrate at new postearthquake levels generally ranged from 10 minutes to two hours, with a median time of 65 minutes and the longest time of $100+$ days (Cromwell Gorge) (Weaver et al., 2019). As a large moving window of 30 days was used for this dataset it is unlikely that fluid changes could be observed within the velocity changes recovered, but it still could be a factor to explain the observed velocity changes.

From these four mechanisms; as our velocity changes are shallow (2.5-5 km deep), and a decrease is observed at all stations across the region (with a larger decrease at stations pairs that cross ruptured faults or have stations on faults), it is likely that damage of the crust from the fault zone and strong ground motions are the mechanisms that contribute the most to our observed seismic velocity decrease and increase.

\subsection{Comparison to Previous Studies}

The most likely mechanisms to have caused velocity changes in this dataset are damage of the crust from the fault zone rupture and subsequent healing and damage of the shallow crust from strong ground shaking. The results found in this study can then be compared to previous studies that also have seismic velocity changes resulting from similar mechanisms. A review paper by Bonnefoy-Claudet et al. (2006) provides a review for studies earlier than 2006. The table below, (Table 6.3) provides an incomplete list of ambient noise studies post 2006 and relevant to this thesis with the results they procured from their datasets. Two papers from Table 6.3 will be compared to each of the two main mechanisms of seismic velocity change observed 
in this thesis. Often studies experience more than one mechanism to be the cause of velocity changes but in this case we will mainly be focusing on the dominant mechanism. Brenguier et al. (2008a) and Minato et al. (2012) both determined that damage of the shallow crust by strong ground motion was one of the main causes of seismic velocity decrease. Whereas both Taira et al. (2015) and Heckels (2017) found damage of the crust from the fault zone rupture and crack healing to be the dominant mechanism. 


\begin{tabular}{|c|c|c|c|c|c|c|c|c|}
\hline 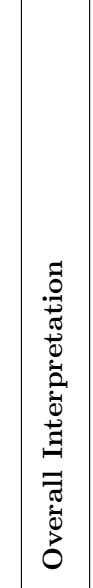 & 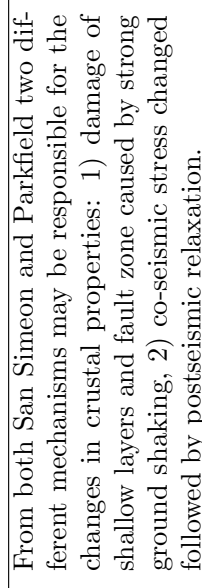 & 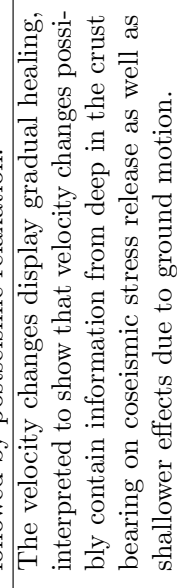 & 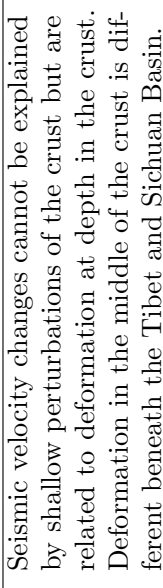 & 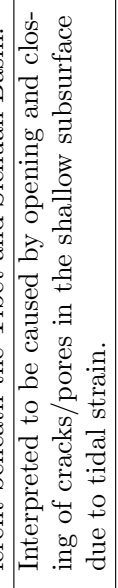 & 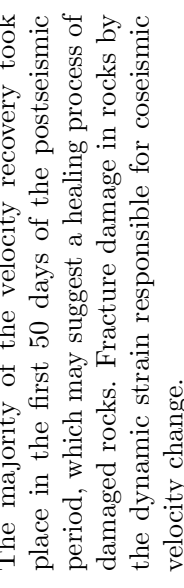 & 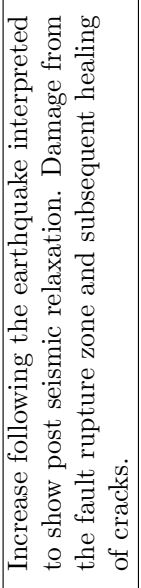 & 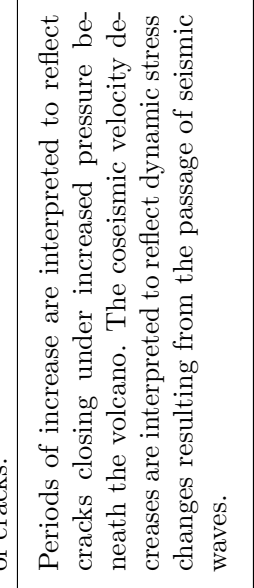 & 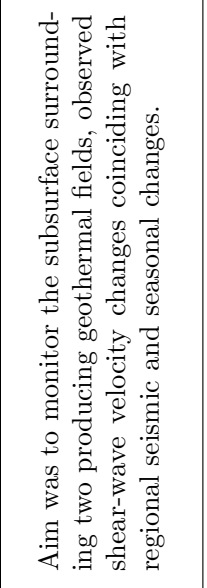 \\
\hline 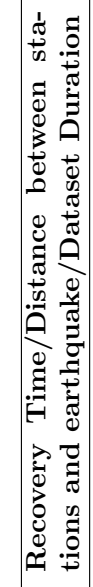 & 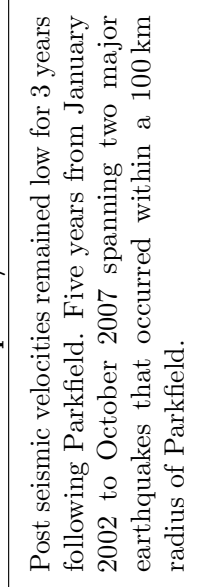 & 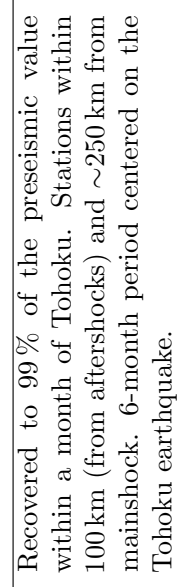 & 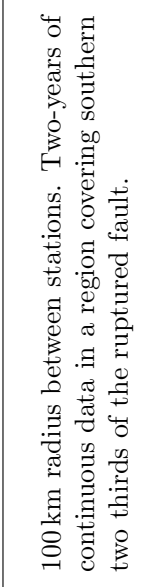 & 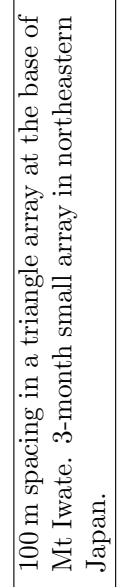 & 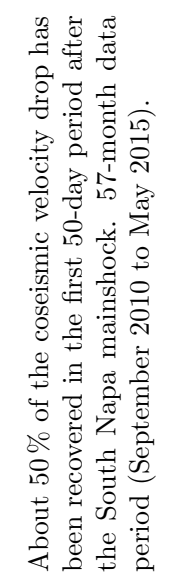 & 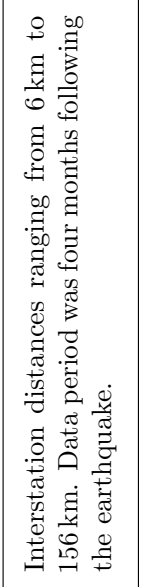 & 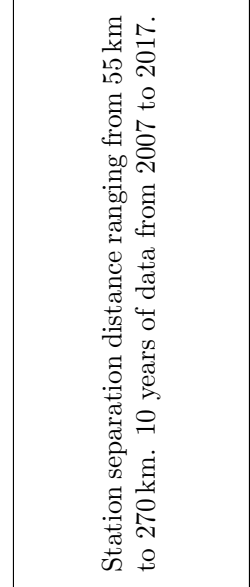 & 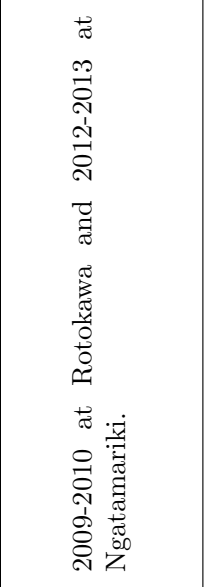 \\
\hline 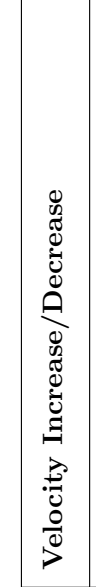 & 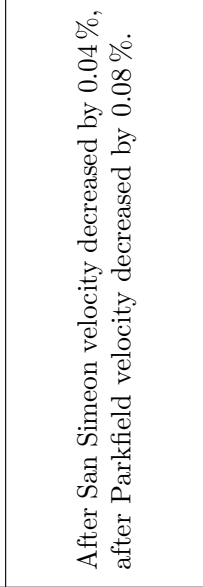 & 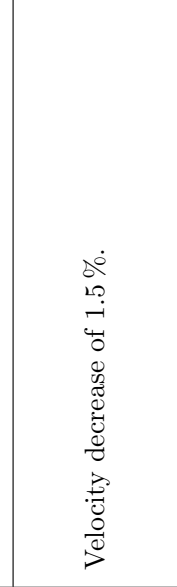 & 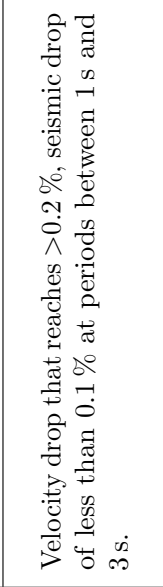 & 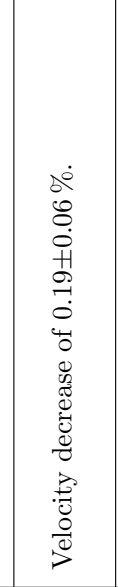 & 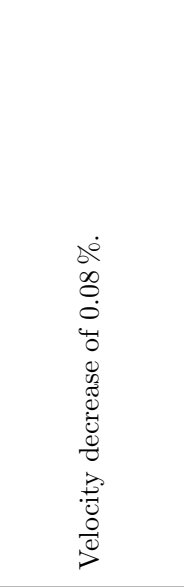 & 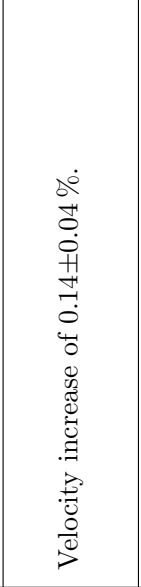 & 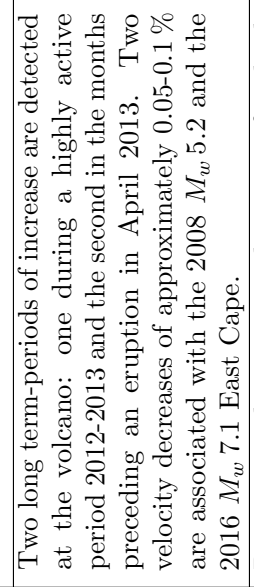 & 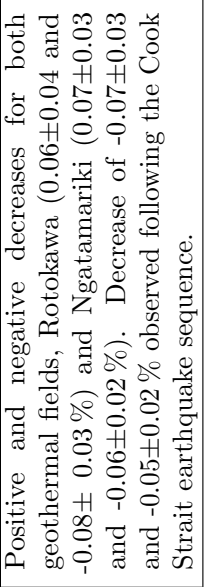 \\
\hline 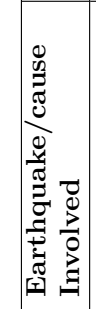 & 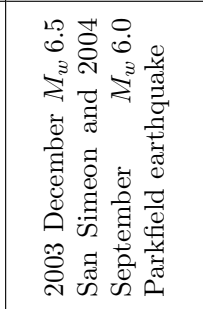 & 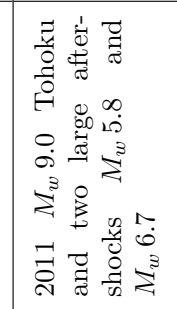 & 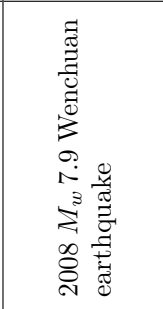 & 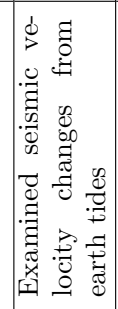 & 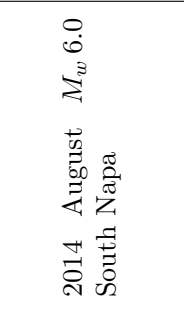 & 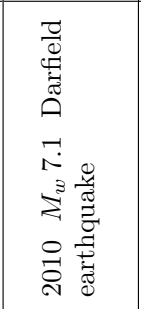 & 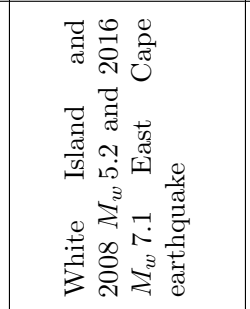 & 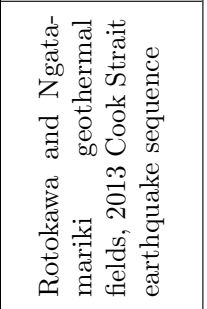 \\
\hline 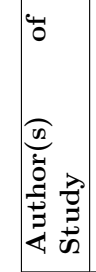 & 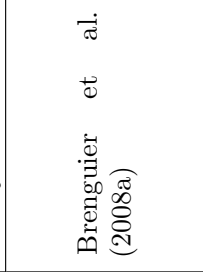 & 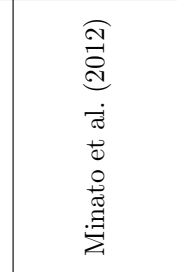 & 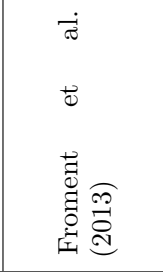 & 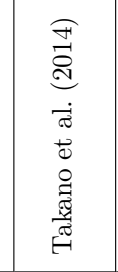 & 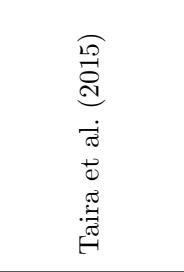 & 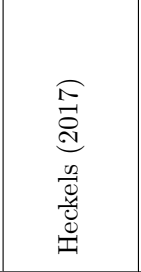 & 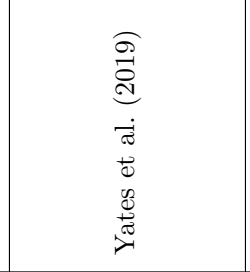 & 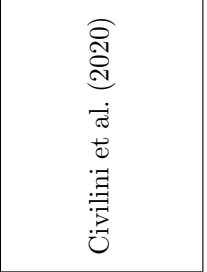 \\
\hline
\end{tabular}


Brenguier et al. (2008a) observed a $0.04 \%$ seismic velocity decrease after the 2003 $M_{w}$ 6.5 San Simeon earthquake. Following the San Simeon earthquake, GPS measurements showed that there were no substantial slip detected along the San Andreas Fault in the Parkfield area. This suggested that the velocity changes detected may be related to co-seismic damage in the shallow layers caused by strong ground shaking $(\sim 0.15 \mathrm{~g})$ (Brenguier et al., 2008a). In the following seven months after the San Simeon earthquake, velocities in the Parkfield area returned to pre-earthquake values before the $2004 M_{w} 6.0$ Parkfield earthquake occurred. This earthquake caused a $0.08 \%$ seismic velocity decrease which was governed by the postseismic stress relaxation within deeper parts of the fault zone and surrounding region (Brenguier et al., 2008a). Minato et al. (2012) measured a 1.5\% seismic velocity decrease in the area nearest the mainshock following the $2011 M_{w} 9.0$ Tohoku earthquake. This velocity change contains information on the results of stress deep underground in addition to shallower effects (around 200m deep) due to damage to the near-surface from strong ground motion (Minato et al., 2012).

Both Brenguier et al. (2008a) and Minato et al. (2012) show that strong ground motions can have shallow effects and stress changes can affect deeper parts of the fault zone. These results align well with this study as both demonstrate how strong ground shaking can affect velocity changes. However, deeper changes that they both observed were not observed in this study, so stress induced changes are not likely a mechanism present here.

Taira et al. (2015) observed a $0.08 \%$ velocity decrease following the $2014 M_{w} 6.0$ South Napa earthquake. Taira et al. (2015) describes the decrease as being a result of fracture damage in rocks by the dynamic strain responsible for the coseismic velocity change. Taira et al. (2015) also measured a $50 \%$ recovery $(0.04 \%)$ in the velocity in the first 50-days following the South Napa earthquake and suggests the postseismic velocity recovery is caused by a healing process for damaged rocks. Heckels (2017) also found a similar increase of $0.14 \pm 0.04 \%$ in the four months following the 2010 $M_{w} 7.1$ Darfield earthquake and interpreted it to show post seismic relaxation and 
healing of cracks.

Following the 2016 Kaikōura earthquake, a velocity decrease of $0.24 \pm 0.02 \%$ was observed on the vertical-vertical component. This value is larger than Brenguier et al. (2008b) and Taira et al. (2015) observed for earthquakes with a lower magnitude and smaller than Minato et al. (2012) observed for a larger magnitude earthquake. Our study aligns well with shallow observations for Brenguier et al. (2008a) and Minato et al. (2012) determining that near-surface strong ground motions were the main mechanism for velocity decreases. Following the velocity decrease, in the oneand-a-half-years after the Kaikōura earthquake, a velocity increase of $0.13 \pm 0.02 \%$ was observed. This occurred over a slower time frame than both Heckels (2017) and Taira et al. (2015) found but can be interpreted as another mechanism: damage from the fault-zone rupture and subsequent healing.

\subsection{Geological Hazard Monitoring}

Using ambient noise to detect and understand more about geological hazards is an ever developing avenue of research. It is uncertain to say whether ambient noise technology could be used to detect precursor events as they could be difficult to interpret. In the case of this study, the only large magnitude event that was able to cause a significant (greater than $0.1 \%$ ) velocity decrease was the $2016 M_{w} 7.8$ Kaikōura earthquake. Events on the order of magnitude 5.6-6.6 were difficult to determine whether they caused a decrease in the seismic velocity (see Figure 6.8). Civilini et al. (2020) results suggest that using more stations with more components could create better resolution. This was not applicable to this dataset but could help in future studies.

Ambient noise could potentially be used to understand the post-seismic healing processes. Knowing more about the healing process would lead to a better understanding of the cracks and the fluid dynamics within a region. With this study, in the one-and-a-half-years following the Kaikoura earthquake, a steady increase 
in seismic velocity of $0.13 \pm 0.02 \%$ was observed. This amount of healing was also observed by Heckels (2017) following the $M_{w} 7.1$ Darfield earthquake $(0.14 \pm 0.04 \%$ over four months) and by Brenguier et al. (2008a) following the $2003 M_{w} 6.5$ San Simeon and $2004 M_{w} 6.0$ Parkfield earthquake in California with an (elevated velocity reduction for three years following these two earthquakes). Another study monitoring spatial and temporal seismic velocity changes on Kyushu Island during the 2016 Kumamoto earthquake found that continuous monitoring of faults and volcanoes could be useful (Nimiya et al., 2017). This study suggested that the identification of future earthquakes could be achieved by measuring velocity changes due to slow slip or foreshocks identified through crustal-scale monitoring.

Studies suggest that ambient noise could also be useful to predict earthquake ground motions. Ambient noise Green's functions can be used to increase our knowledge of the velocity structure of three-dimensional crustal structures which is important for accurate simulations of long-period strong ground motions (Prieto and Beroza, 2008). To generate the surface-wave impulse response or the Green's function, the coherent signal from one station to another is recovered. The impulse response function between a pair of stations is then recoverable by cross-correlating simultaneous time series and stacking (averaging) over time (Denolle et al., 2013). Impulse response functions reproduce waveform shapes and relative amplitudes of earthquakes from nearby stations (Denolle et al., 2014). Impulse response functions can be corrected from a surface point-force source to a double-couple source at depth and then the comparison between comparing computed seismograms with earthquake waveforms improves (Denolle et al., 2014). Once comparable simulated earthquakes can be generated to predict long-period strong ground motion for future earthquakes in regions subject to high seismic risk (Denolle et al., 2014).

This above method has been used in California by Prieto and Beroza (2008), Denolle et al. (2013) and Denolle et al. (2014) to simulate strong ground motions and to understand more about basin amplification. (Denolle et al., 2014) produce a range of magnitude 7 scenario earthquakes on the southern San Andreas fault and compare 
strong ground motion predictions with simulations. They found strong amplification and coupling of source and structure effects from the simulated strong ground motions and they also predicted substantially different shaking patterns across the Los Angeles Basin (Denolle et al., 2014). This is a powerful tool in hazard analysis as it allows knowledge to be gained about regions that would be more or less affected by different magnitude earthquakes. Prieto et al. (2010) also demonstrates that an impulse response function can be generated for a multi-storey building from ambient noise. Monitoring civil structures for responses to earthquake motions can be fundamental in reducing seismic risk (Prieto et al., 2010). Predicting accurate strong ground motions can be crucial for accurate earthquake hazard analysis on both a regional scale as well as for more specific locations, such as buildings.

Although using ambient noise to detect pre-cursors is relatively unstudied, using ambient noise to simulate strong ground motions has proved promising in California. This would allow for simulations of New Zealand to be generated to determine what the effects of large earthquakes would be. As the ambient noise process is also "active", in that seismologists can design experiments to determine station-to-station Green's functions, this allows for ground motion estimates to target areas of particular concern (Prieto and Beroza, 2008). 


\section{Chapter 7}

\section{Conclusions}

This thesis presents the results from velocity variations recovered using ambient noise methods between 2012 to 2018 from the 2016 Kaikōura earthquake and other large magnitude events. In Chapter 1, three primary objectives were defined, and are shown below:

1. Investigate seismic velocity changes in the Kaikôura region and their connection to the 2016 Kaikōura earthquake. Attempt to determine if there was a change before/after the earthquake.

2. Determine how this change varied across the region.

3. Consider if ambient noise can lead to improved detection and understanding of geological hazard and if this could result in enhanced assessment and modelling of New Zealand's geohazard risk.

To determine changes after the Kaikōura earthquake, analysis was first used for a two-year period from the $1^{\text {st }}$ of January 2016 to the end of the available dataset, the $28^{\text {th }}$ of February 2018. A full duration of seven years was used later when more data was available and the ambient noise processing parameters had been determined. These same processing parameters that were first applied to the vertical-vertical component were applied to all cross-components. All of the cross-components showed a decrease in seismic velocity following the 2016 Kaikōura earthquake, however each 
result varied. In this chapter, we summarise the findings of this thesis (Section 7.1) and recommend potential areas of future work (Section 7.2).

\subsection{Summary of Findings}

Sections 7.1.1, 7.1.2, and 7.1.3 develop the key findings of this thesis in direct relation to the three primary objectives.

\subsubsection{Kaikōura earthquake}

In the seven years of data analysed, one period of long-term seismic velocity decrease is detected following the 2016 Kaikōura earthquake. Following this seismic decrease, there is a gradual increase over one-and-a-half-years, which is interpreted to reflect cracks closing as part of a healing post-seismic process.

A seismic velocity decrease of $0.24 \pm 0.02 \%$ was observed following the 2016 Kaikoura earthquake on the vertical-vertical component averaged over eight stations (see Figure 6.8). The remaining eight cross-components all showed a slightly smaller seismic decrease with an average value of $0.22 \pm 0.05 \%$. Following the Kaikōura earthquake there is a steady increase of $0.13 \pm 0.02 \%$ over a one-and-a-half-year period. This indicates that prior to the earthquake, seismic velocity was at a steady state until it was perturbed by the Kaikōura earthquake, and seismic velocities rapidly decreased over all stations. After seismic velocities had reached a minimum value of $-0.21 \%$ for the vertical-vertical component, a steady increase in seismic velocities occurred. This has been interpreted to be caused by cracks forming due to the Kaikōura earthquake and then steadily closing as a part of crack healing process, as pressure increased and cemented around the faults in the Kaikōura region. As many as 21 faults ruptured (Stirling et al., 2017), with seismic velocity changes recoverable over a large region. Due to the large rupture area, damage of the crust from strong ground motions is another likely mechanism for velocity changes. 


\subsubsection{Regional Seismic Changes}

Prior to the 2016 Kaikōura earthquake, in 2013, a doublet event occurred, with an earthquake occurring in the Cook Strait with a magnitude $M_{w} 6.5$ in July 2013, and a magnitude $M_{w}$ 6.6 Lake Grassmere earthquake in August 2013. Despite both of these earthquakes being quite large, neither of them were interpreted to have had an effect on the seismic velocity in this dataset (Figure 6.8). This has been interpreted to be a result of all of the stations that contained data for this period not being sensitive enough as Civilini et al. (2020), in a geothermal field $\sim 400 \mathrm{~km}$ away, recovered a velocity drop of $0.06 \%$ following the Cook Strait earthquake sequence.

Another significant period occurred in the middle of the dataset (earthquakes 3 and 10 in Figure 6.6) where two deep events (52 km and $48 \mathrm{~km}$ respectively) occurred in April $2015\left(M_{w} 6.2\right)$ and in February $2016\left(M_{w} 5.7\right)$. Both of these events resulted in a small seismic decrease of $0.1 \pm 0.02 \%$. We suspect that these events did not cause a larger seismic decrease due to the events being relatively deep compared to the Kaikōura earthquake, which was quite shallow at $15 \mathrm{~km}$ deep. We also found that generally the velocity decrease was larger closer to the earthquake or ruptured fault. A decrease of $\sim 1 \%$ was observed for IKR-MLF compared to a velocity decrease of $\sim 0.15 \%$ at SJQ-WJM (Figure 6.4). Where both IKR and MLF are located $<10 \mathrm{~km}$ away from ruptured faults and SJQ and WJM are $>100 \mathrm{~km}$ away from ruptured faults (and further, $\sim 120 \mathrm{~km}$ from the mainshock). This suggests that location proximity to ruptured faults and the earthquake does have an effect on the velocity change.

\subsubsection{Geological Hazard}

Ambient noise could potentially be used to monitor earthquakes in real time. The dataset presented in this thesis shows that interpreting changes in seismic velocity before the Kaikōura earthquake would be difficult. As this was the largest earthquake in the time period analysed, any obvious markers of a change would most likely be 
viewed before this earthquake. In the smaller earthquakes in 2015, only a slight decrease in seismic velocity can be seen. These small changes would be quite difficult to distinguish from long-term fluctuations and would not be useful to predict if larger events are about to occur.

Although using ambient noise to measure real-time changes will be challenging, it could be possible to analyse the recovery of faults in the region following a large earthquake. This would allow for a better understanding of fractures and the movement of water within the fractures following a large magnitude earthquake. In this study, the magnitude 6.2 and 5.7 earthquakes in 2015 and 2016 did not have a great enough magnitude or were shallow enough to produce a clear decrease in seismic velocity and a gradual healing as the 2016 Kaikōura earthquake did. This indicates that for a healing process to be observed, the earthquake magnitude must be quite large $\left(M_{w}>7.0\right)$, shallow $($ depth $<40 \mathrm{~km})$ and at distances of $<100 \mathrm{~km}$ between the stations and ruptured faults or earthquake.

\subsection{Recommendations for Further Work}

This thesis demonstrates how ambient noise made it possible to generate results from the DPRI short-period network, which although limited in the station duration and quality, seismic velocity changes were still able to be measured. Recommendations for future work are outlined below. These suggestions hope to provide guidance on how ambient noise can be used to understand the Kaikōura region and how these results can lead to a better understanding of geologic hazard.

- Increase the DPRI data period: This dataset finishes at the end of February 2018, showing a gradual increase in seismic velocity. Monitoring for a later period would show a more complete recovery back to pre-earthquake seismic velocity change values. This would give a better understanding of the duration of a crack healing process for a complex multi-fault rupture. It would also be interesting to see how the seismic velocity changes varied with distance away 
from the fault. This thesis did cover this, but a more precise result would be obtained by using more stations.

- Combine results with GeoNet stations: The majority of the stations in this dataset had faulty stations and often did not have data for particular components for large periods of time. The accuracy of the results presented in this thesis could be increased if the short-period results are combined with the broad-band GeoNet stations (Jacobs and Savage, 2019). This would also allow for better analysis of Rayleigh and particularly Love waves, as the GeoNet stations will be able to have the horizontal components rotated. Having broad-band stations as well as short-period stations would also allow for better recovery of deeper surface waves.

- Analysing seasonal trends: Within this thesis, seven years of data (2012 - 2018) was examined for major changes in seismic velocity following large magnitude events. This covered a large region and as such not everything of interest has been discussed in this thesis. Studying seasonal trends such as correlations between weather and rainfall would be interesting to see if it affected the velocity changes greatly. Another interesting avenue of research would be if the stations closer to the ocean are more affected by wave energy (changes related to source characteristics) and to what extent it affects the seismic velocity changes. As this avenue has yet to be explored with this dataset, we believe that there still may be more interesting features to be found by ambient noise. 
7.2. Recommendations for Further Work 


\section{Bibliography}

K. Aki. Space and time spectra of stationary stochastic waves, with special reference to microtremors. Bulletin of the Earthquake Research Institute, 35:415-456, 1957.

K. Aki. Analysis of the seismic coda of local earthquakes as scattered waves. Journal of Geophysical Research, 74(2):615-631, 1969. ISSN 0148-0227.

K. Aki. Scattering and attenuation of shear waves in the lithosphere. Journal of Geophysical Research: Solid Earth, 85(B11):6496-6504, 1980. ISSN 2156-2202.

K. Aki and B. Chouet. Origin of coda waves: source, attenuation, and scattering effects. Journal of Geophysical Research, 80(23):3322-3342, 1975. ISSN 2156-2202.

K. Aki and P. G. Richards. Quantitative Seismology. University Science Books, 2nd edition, 2002. ISBN 0935702962.

M. W. Asten. Geological control on the three-component spectra of Rayleigh-wave microseisms. Bulletin of the Seismological Society of America, 68(6):1623-1636, 1978. ISSN 1943-3573.

M. W. Asten and J. D. Henstridge. Array estimators and the use of microseisms for reconnaissance of sedimentary basins. Geophysics, 49(11):1828-1837, 1984. ISSN 0016-8033.

W. H. Bakun and C. G. Bufe. Shear-wave attenuation along the San Andreas fault zone in central California. Bulletin of the Seismological Society of America, 65(2): 439-459, 1975. ISSN 1943-3573.

G. D. Bensen, M. H. Ritzwoller, M. P. Barmin, A. L. Levshin, F. Lin, M. P. Moschetti, 
N. M. Shapiro, and Y. Yang. Processing seismic ambient noise data to obtain reliable broad-band surface wave dispersion measurements. Geophysical Journal International, 169(3):1239-1260, 2007. ISSN 1365-246X.

S. Bonnefoy-Claudet, F. Cotton, and P.-Y. Bard. The nature of noise wavefield and its applications for site effects studies: A literature review. Earth-Science Reviews, 79(3-4):205-227, 2006. ISSN 0012-8252.

D. C. Booth, S. Crampin, J. H. Lovell, and J. Chiu. Temporal changes in shear wave splitting during an earthquake swarm in Arkansas. Journal of Geophysical Research: Solid Earth, 95(B7):11151-11164, 1990. ISSN 0148-0227.

B. A. Bradley, H. N. T. Razafindrakoto, and M. Nazer. Strong ground motion observations of engineering interest from the 14 November 2016 Mw7. 8 Kaikoura, New Zealand earthquake. 2017.

J. D. Bradshaw. Cretaceous geotectonic patterns in the New Zealand region. Tectonics, 8(4):803-820, 1989. ISSN 0278-7407.

F. Brenguier, N. M. Shapiro, M. Campillo, A. Nercessian, and V. Ferrazzini. 3-D surface wave tomography of the Piton de la Fournaise volcano using seismic noise correlations. Geophysical Research Letters, 34(2), 2007. ISSN 1944-8007.

F. Brenguier, M. Campillo, C. Hadziioannou, N. M. Shapiro, R. M. Nadeau, and E. Larose. Postseismic relaxation along the San Andreas fault at Parkfield from continuous seismological observations. Science, 321(5895):1478-1481, 2008a. ISSN 0036-8075.

F. Brenguier, N. M. Shapiro, M. Campillo, V. Ferrazzini, Z. Duputel, O. Coutant, and A. Nercessian. Towards forecasting volcanic eruptions using seismic noise. Nature Geoscience, 1(2):126, 2008b. ISSN 1752-0908.

F. Brenguier, M. Campillo, T. Takeda, Y. Aoki, N. M. Shapiro, X. Briand, K. Emoto, and H. Miyake. Mapping pressurized volcanic fluids from induced crustal seismic velocity drops. Science, 345(6192):80-82, 2014. 
M. Campillo. Phase and Correlation in Random Seismic Fields and the Reconstruction of the Green Function. Pure and Applied Geophysics, 163(2-3):475-502, 2006. ISSN 0033-4553.

M. Campillo and A. Paul. Long-range correlations in the diffuse seismic coda. Science, 299(5606):547-549, 2003. ISSN 0036-8075.

S. Cesca, Y. Zhang, V. Mouslopoulou, R. Wang, J. Saul, M. Savage, S. Heimann, S.-K. Kufner, O. Oncken, and T. Dahm. Complex rupture process of the Mw 7.8, 2016, Kaikoura earthquake, New Zealand, and its aftershock sequence. Earth and Planetary Science Letters, 478:110-120, 2017.

C. C. Chiew, Y. B. Cheah, C. G. Tan, and T. L. Lau. Estimation of soft sediment thickness in Kuala Lumpur based on microtremor observation data. In AIP Conference Proceedings, volume 1892, page 120002. AIP Publishing, 2017. ISBN 0735415749.

F. Civilini. Determining seismic shear-velocity from ambient noise sources at regional and local scales. PhD thesis, Victoria University of Wellington, 2018.

F. Civilini, M. K. Savage, and J. Townend. Shear wave velocity changes induced by earthquakes and rainfall at the Rotokawa and Ngatamariki geothermal fields, Taupō Volcanic Zone, New Zealand. Geophysical Journal International, 221(1): 97-114, 2020. ISSN 0956-540X.

J. F. Claerbout. Synthesis of a layered medium from its acoustic transmission response. Geophysics, 33(2):264-269, 1968. ISSN 0016-8033.

K. J. Clark, E. K. Nissen, J. D. Howarth, I. J. Hamling, J. J. Mountjoy, W. F. Ries, K. Jones, S. Goldstien, U. A. Cochran, and P. Villamor. Highly variable coastal deformation in the 2016 Mw7. 8 Kaikōura earthquake reflects rupture complexity along a transpressional plate boundary. Earth and Planetary Science Letters, 474: 334-344, 2017.

D. Clarke, L. Zaccarelli, N. M. Shapiro, and F. Brenguier. Assessment of resolution 
and accuracy of the Moving Window Cross Spectral technique for monitoring crustal temporal variations using ambient seismic noise. Geophysical Journal International, 186(2):867-882, 2011.

H. A. Cowan. The north Canterbury earthquake of September 1, 1888. Journal of the Royal Society of New Zealand, 21(1):1-12, 1991. ISSN 0303-6758.

A. Curtis, P. Gerstoft, H. Sato, R. Snieder, and K. Wapenaar. Seismic interferometry-Turning noise into signal. The Leading Edge, 25(9):1082-1092, 2006. ISSN 1070-485X.

E. Daskalakis, C. P. Evangelidis, J. Garnier, N. S. Melis, G. Papanicolaou, and C. Tsogka. Robust seismic velocity change estimation using ambient noise recordings. Geophysical Journal International, 205(3):1926-1936, 2016. ISSN 1365-246X.

M. A. Denolle, E. M. Dunham, G. A. Prieto, and G. C. Beroza. Ground motion prediction of realistic earthquake sources using the ambient seismic field. Journal of Geophysical Research: Solid Earth, 118(5):2102-2118, 2013. ISSN 2169-9313.

M. A. Denolle, E. M. Dunham, G. A. Prieto, and G. C. Beroza. Strong ground motion prediction using virtual earthquakes. Science, 343(6169):399-403, 2014. ISSN 0036-8075.

A. Derode, E. Larose, M. Tanter, J. De Rosny, A. Tourin, M. Campillo, and M. Fink. Recovering the Green's function from field-field correlations in an open scattering medium (L). The Journal of the Acoustical Society of America, 113(6):2973-2976, 2003. ISSN 0001-4966.

D. Draganov, K. Wapenaar, W. Mulder, J. Singer, and A. Verdel. Retrieval of reflections from seismic background-noise measurements. Geophysical Research Letters, 34(4), 2007. ISSN 0094-8276.

Z. Duputel and L. Rivera. Long-period analysis of the 2016 Kaikoura earthquake. Physics of the Earth and Planetary Interiors, 265:62-66, 2017. ISSN 0031-9201. 
T. L. Duvall Jr, S. M. Jeffferies, J. W. Harvey, and M. A. Pomerantz. Time-distance helioseismology. Nature, 362(6419):430, 1993. ISSN 1476-4687.

D. Eberhart-Phillips and B. Fry. Joint local earthquake and teleseismic inversion for 3-D velocity and Q in New Zealand. Physics of the Earth and Planetary Interiors, 283:48-66, 2018. ISSN 0031-9201.

J. Frechet, L. Martel, L. Nikolla, and G. Poupinet. Application of the cross-spectral moving-window technique (CSMWT) to the seismic monitoring of forced fluid migration in a rock mass. In International Journal of Rock Mechanics and Mining Sciences $\&$ Geomechanics Abstracts, volume 26, pages 221-233. Elsevier, 1989. ISBN 0148-9062.

A. Friedrich, F. Krueger, and K. Klinge. Ocean-generated microseismic noise located with the Gräfenberg array. Journal of Seismology, 2(1):47-64, 1998. ISSN 13834649.

B. Froment, M. Campillo, J. H. Chen, and Q. Y. Liu. Deformation at depth associated with the 12 May 2008 Mw 7.9 Wenchuan earthquake from seismic ambient noise monitoring. Geophysical Research Letters, 40(1):78-82, 2013. ISSN 0094-8276.

P. M. Giles, T. L. Duvall Jr, P. H. Scherrer, and R. S. Bogart. A subsurface flow of material from the sun's equator to its poles. Nature, 390(6655):52, 1997. ISSN 1476-4687.

K. Gledhill, J. Ristau, M. Reyners, B. Fry, and C. Holden. The Darfield (Canterbury, New Zealand) Mw 7.1 earthquake of September 2010: A preliminary seismological report. Seismological Research Letters, 82(3):378-386, 2011.

R. Grapes, T. Little, and G. Downes. Rupturing of the Awatere Fault during the 1848 October 16 Marlborough earthquake, New Zealand: historical and present day evidence. New Zealand Journal of Geology and Geophysics, 41(4):387-399, 1998. ISSN 0028-8306.

J. C. Groos, S. Bussat, and J. R. R. Ritter. Performance of different processing 
schemes in seismic noise cross-correlations. Geophysical Journal International, 188 (2):498-512, 2012. ISSN 1365-246X.

B. Gutenberg. Observations and theory of microseisms. In Compendium of Meteorology, pages 1303-1311. Springer, 1951.

B. Gutenberg. Microseisms. In Advances in Geophysics, volume 5, pages 53-92. Elsevier, 1958. ISBN 0065-2687.

C. Hadziioannou, E. Larose, O. Coutant, P. Roux, and M. Campillo. Stability of monitoring weak changes in multiply scattering media with ambient noise correlation: Laboratory experiments. The Journal of the Acoustical Society of America, 125(6):3688-3695, 2009. ISSN 0001-4966.

C. Hadziioannou, E. Larose, A. Baig, P. Roux, and M. Campillo. Improving temporal resolution in ambient noise monitoring of seismic wave speed. Journal of Geophysical Research: Solid Earth, 116(B7), 2011. ISSN 0148-0227.

I. J. Hamling, S. Hreinsdóttir, K. Clark, J. Elliott, C. Liang, E. Fielding, N. Litchfield, P. Villamor, L. Wallace, and T. J. Wright. Complex multifault rupture during the 2016 M w 7.8 Kaikōura earthquake, New Zealand. Science, 356(6334):eaam7194, 2017.

S.-C. Han, C. K. Shum, M. Bevis, C. Ji, and C.-Y. Kuo. Crustal dilatation observed by GRACE after the 2004 Sumatra-Andaman earthquake. Science, 313(5787): 658-662, 2006. ISSN 0036-8075.

M. M. Haney, A. J. Hotovec-Ellis, N. L. Bennington, S. De Angelis, and C. Thurber. Tracking changes in volcanic systems with seismic interferometry. Encyclopedia of Earthquake Engineering, pages 1-23, 2014. ISSN 3642361978.

R. Heckels. Using ambient seismic noise to study temporal and spatial surface wave velocity structures and ambient noise field characteristics of central South Island, New Zealand. PhD thesis, Victoria University of Wellington, 2017.

R. E. G. Heckels, M. K. Savage, and J. Townend. Post-seismic velocity changes 
following the $2010 \mathrm{M}$ w 7.1 Darfield earthquake, New Zealand, revealed by ambient seismic field analysis. Geophysical Journal International, 213(2):931-939, 2018.

G. Helffrich, J. Wookey, and I. Bastow. The Seismic Analysis Code: A primer and user's guide. Cambridge University Press, 2013. ISBN 1107045452.

R. B. Herrmann. Computer programs in seismology: An evolving tool for instruction and research. Seismological Research Letters, 84(6):1081-1088, 2013. ISSN 19382057.

G. Hillers, Y. Ben-Zion, M. Campillo, and D. Zigone. Seasonal variations of seismic velocities in the San Jacinto fault area observed with ambient seismic noise. Geophysical Journal International, 202(2):920-932, 2015. ISSN 1365-246X.

M. Hobiger, U. Wegler, K. Shiomi, and H. Nakahara. Coseismic and postseismic elastic wave velocity variations caused by the 2008 Iwate-Miyagi Nairiku earthquake, Japan. Journal of Geophysical Research: Solid Earth, 117(B9), 2012. ISSN 01480227.

M. Hobiger, U. Wegler, K. Shiomi, and H. Nakahara. Single-station cross-correlation analysis of ambient seismic noise: application to stations in the surroundings of the 2008 Iwate-Miyagi Nairiku earthquake. Geophysical Journal International, 198 (1):90-109, 2014. ISSN 1365-246X.

K. Irikura. Prediction of strong acceleration motion using empirical Green's function. In Proceedings of the 7th Japan Earthquake Engineering Symposium, volume 151, pages $151-156,1986$.

K. Jacobs and M. K. Savage. Velocity Changes in Cook Strait Following the 2016 Kaikōura Earthquake. In New Zealand Geosciences 2019 Abstracts. Geosciences Conference, Hamilton, New Zealand, 2019.

R. M. Langridge, W. F. Ries, N. J. Litchfield, P. Villamor, R. J. Van Dissen, D. J. A. Barrell, M. S. Rattenbury, D. W. Heron, S. Haubrock, and D. B. Townsend. 
The New Zealand active faults database. New Zealand Journal of Geology and Geophysics, 59(1):86-96, 2016.

E. Larose, A. Derode, D. Clorennec, L. Margerin, and M. Campillo. Passive retrieval of Rayleigh waves in disordered elastic media. Physical Review E, 72(4):46607, 2005 .

E. Larose, P. Roux, and M. Campillo. Reconstruction of Rayleigh-Lamb dispersion spectrum based on noise obtained from an air-jet forcing. The Journal of the Acoustical Society of America, 122(6):3437-3444, 2007. ISSN 0001-4966.

T. Lecocq. MSNoise Documentation Release 1.4, 2019. URL http://msnoise.org/ doc/MSNoise.pdf.

T. Lecocq, C. Caudron, and F. Brenguier. MSNoise, a python package for monitoring seismic velocity changes using ambient seismic noise. Seismological Research Letters, 85(3):715-726, 2014.

E. Lev, M. D. Long, and R. D. van der Hilst. Seismic anisotropy in Eastern Tibet from shear wave splitting reveals changes in lithospheric deformation. Earth and Planetary Science Letters, 251(3-4):293-304, 2006. ISSN 0012-821X.

Y.-G. Li, J. E. Vidale, K. Aki, F. Xu, and T. Burdette. Evidence of shallow fault zone strengthening after the 1992 M7. 5 Landers, California, earthquake. Science, 279(5348):217-219, 1998. ISSN 0036-8075.

F. Lin, B. Schmandt, and V. C. Tsai. Joint inversion of Rayleigh wave phase velocity and ellipticity using USArray: Constraining velocity and density structure in the upper crust. Geophysical Research Letters, 39(12), 2012. ISSN 0094-8276.

F.-C. Lin, M. H. Ritzwoller, J. Townend, S. Bannister, and M. K. Savage. Ambient noise Rayleigh wave tomography of New Zealand. Geophysical Journal International, 170(2):649-666, 2007. ISSN 1365-246X.

N. Litchfield, A. Benson, A. Bischoff, A. Hatem, A. Barrier, A. Nicol, A. Wandres, B. Lukovic, B. Hall, and C. Gasston. 14th November 2016 M7. 8 Kaikoura 
Earthquake. Summary surface fault rupture traces and displacement measurements. GNS Sci, 10:G21422RC21427C, 2017.

Z. Liu, J. Huang, Z. Peng, and J. Su. Seismic velocity changes in the epicentral region of the 2008 Wenchuan earthquake measured from three-component ambient noise correlation techniques. Geophysical Research Letters, 41(1):37-42, 2014. ISSN 0094-8276.

O. I. Lobkis and R. L. Weaver. On the emergence of the Green's function in the correlations of a diffuse field. The Journal of the Acoustical Society of America, 110(6):3011-3017, 2001. ISSN 0001-4966.

O. I. Lobkis and R. L. Weaver. Coda-wave interferometry in finite solids: Recovery of P-to-S conversion rates in an elastodynamic billiard. Physical Review Letters, 90(25):254302, 2003.

D. A. Lockner, J. B. Walsh, and J. D. Byerlee. Changes in seismic velocity and attenuation during deformation of granite. Journal of Geophysical Research, 82 (33):5374-5378, 1977. ISSN 0148-0227.

M. S. Longuet-Higgins. A theory of the origin of microseisms. Philosophical Transactions of the Royal Society of London. Series A, Mathematical and Physical Sciences, 243(857):1-35, 1950. ISSN 0080-4614.

A. E. H. Love. A Treatise on the Mathematical Theory of Elasticity, volume 105. Cambridge university press, 1927. ISBN 1107618096. doi: 10.1038/105511a0.

D. Marsan, J. Weiss, E. Larose, and J.-P. Métaxian. Sea-ice thickness measurement based on the dispersion of ice swell. The Journal of the Acoustical Society of America, 131(1):80-91, 2012. ISSN 0001-4966.

J. Milne. Earthquakes and other earth movements, volume 56. D. Appleton, 1886.

S. Minato, T. Tsuji, S. Ohmi, and T. Matsuoka. Monitoring seismic velocity change caused by the 2011 Tohoku-oki earthquake using ambient noise records. Geophysical Research Letters, 39(9), 2012. 
A. Mordret, M. Landès, N. M. Shapiro, S. C. Singh, P. Roux, and O. I. Barkved. Nearsurface study at the Valhall oil field from ambient noise surface wave tomography. Geophysical Journal International, 193(3):1627-1643, 2013. ISSN 1365-246X.

H. Nimiya, T. Ikeda, and T. Tsuji. Spatial and temporal seismic velocity changes on Kyushu Island during the 2016 Kumamoto earthquake. Science Advances, 3(11): e1700813, 2017. ISSN 2375-2548.

T. Nishimura, N. Uchida, H. Sato, M. Ohtake, S. Tanaka, and H. Hamaguchi. Temporal changes of the crustal structure associated with the M6.1 earthquake on September 3, 1998, and the volcanic activity of Mount Iwate, Japan. Geophysical Research Letters, 27(2):269-272, 2000. ISSN 0094-8276.

A. Nur. Effects of stress on velocity anisotropy in rocks with cracks. Journal of Geophysical Research, 76(8):2022-2034, 1971. ISSN 0148-0227.

A. Nur and G. Simmons. Stress-induced velocity anisotropy in rock: An experimental study. Journal of Geophysical Research, 74(27):6667-6674, 1969.

A. Obermann, T. Kraft, E. Larose, and S. Wiemer. Potential of ambient seismic noise techniques to monitor the St. Gallen geothermal site (Switzerland). Journal of Geophysical Research: Solid Earth, 120(6):4301-4316, 2015. ISSN 2169-9313.

T. Okada, Y. Iio, S. Matsumoto, S. Bannister, S. Ohmi, S. Horiuchi, T. Sato, T. Miura, J. Pettinga, and F. Ghisetti. Comparative tomography of reverse-slip and strike-slip seismotectonic provinces in the northern South Island, New Zealand. Tectonophysics, 2019. ISSN 0040-1951.

J. Peterson. Observations and modeling of seismic background noise. Technical report, US Geological Survey, 1993.

P. Poli, H. A. Pedersen, M. Campillo, and P. W. Group. Noise directivity and group velocity tomography in a region with small velocity contrasts: the northern Baltic shield. Geophysical Journal International, 192(1):413-424, 2012. ISSN 1365-246X.

G. Poupinet, W. L. Ellsworth, and J. Frechet. Monitoring velocity variations in the 
crust using earthquake doublets: An application to the Calaveras Fault, California. Journal of Geophysical Research: Solid Earth, 89(B7):5719-5731, 1984. ISSN 0148-0227.

G. A. Prieto and G. C. Beroza. Earthquake ground motion prediction using the ambient seismic field. Geophysical Research Letters, 35(14), 2008. ISSN 0094-8276.

G. A. Prieto, J. F. Lawrence, A. I. Chung, and M. D. Kohler. Impulse response of civil structures from ambient noise analysis. Bulletin of the Seismological Society of America, 100(5A):2322-2328, 2010. ISSN 1943-3573.

A. Ratdomopurbo and G. Poupinet. Monitoring a temporal change of seismic velocity in a volcano: Application to the 1992 eruption of Mt. Merapi (Indonesia). Geophysical Research Letters, 22(7):775-778, 1995. ISSN 0094-8276.

M. Rattenbury, D. Townsend, and M. Johnston. Geology of the Kaikoura Area, volume 1. Institute of Geological \& Nuclear Sciences, 2006. ISBN 0478099258.

L. Rayleigh. On waves propagated along the plane surface of an elastic solid. Proceedings of the London Mathematical Society, 1(1):4-11, 1885. ISSN 0024-6115.

J. Rhie and B. Romanowicz. Excitation of Earth's continuous free oscillations by atmosphere-ocean-seafloor coupling. Nature, 431(7008):552, 2004. ISSN 1476-4687.

J. E. Rickett and J. F. Claerbout. Calculation of the sun's acoustic impulse response by multi-dimensional spectral factorization. In Helioseismic Diagnostics of Solar Convection and Activity, pages 203-210. Springer, 2000.

M. H. Ritzwoller and A. L. Levshin. Eurasian surface wave tomography: Group velocities. Journal of Geophysical Research: Solid Earth, 103(B3):4839-4878, 1998. ISSN 0148-0227.

P. Roux. Passive seismic imaging with directive ambient noise: application to surface waves and the San Andreas Fault in Parkfield, CA. Geophysical Journal International, 179(1):367-373, 2009. ISSN 1365-246X.

P. Roux, W. A. Kuperman, and N. Group. Extracting coherent wave fronts from 
acoustic ambient noise in the ocean. The Journal of the Acoustical Society of America, 116(4):1995-2003, 2004. ISSN 0001-4966.

J. L. Rubinstein and G. C. Beroza. Evidence for widespread nonlinear strong ground motion in the Mw 6.9 Loma Prieta earthquake. Bulletin of the Seismological Society of America, 94(5):1595-1608, 2004. ISSN 1943-3573.

K. G. Sabra, P. Gerstoft, P. Roux, W. A. Kuperman, and M. C. Fehler. Extracting time-domain Green's function estimates from ambient seismic noise. Geophysical Research Letters, 32(3), 2005. ISSN 0094-8276.

M. K. Savage. Biennial Contestable Grants Programme - 2018, 2018.

M. K. Savage. EQC Progress Report 18/755. Technical report, Victoria University of Wellington, 2019.

M. K. Savage, F. Lin, and J. Townend. Ambient noise cross-correlation observations of fundamental and higher-mode Rayleigh wave propagation governed by basement resonance. Geophysical Research Letters, 40(14):3556-3561, 2013.

K. Sawazaki, H. Sato, H. Nakahara, and T. Nishimura. Time-lapse changes of seismic velocity in the shallow ground caused by strong ground motion shock of the 2000 Western-Tottori earthquake, Japan, as revealed from coda deconvolution analysis. Bulletin of the Seismological Society of America, 99(1):352-366, 2009. ISSN 1943-3573.

M. Schimmel and H. Paulssen. Noise reduction and detection of weak, coherent signals through phase-weighted stacks. Geophysical Journal International, 130(2): 497-505, 1997. ISSN 1365-246X.

C. Sens-Schönfelder and U. Wegler. Passive image interferometry for monitoring crustal changes with ambient seismic noise. Comptes Rendus Geoscience, 343(8-9): 639-651, 2011. ISSN 1631-0713.

C. Sens-Schönfelder and U. Wegler. Passive image interferometry and seasonal 
variations of seismic velocities at Merapi Volcano, Indonesia. Geophysical Research Letters, 33(21), 2006. ISSN 1944-8007.

N. M. Shapiro and M. Campillo. Emergence of broadband Rayleigh waves from correlations of the ambient seismic noise. Geophysical Research Letters, 31(7), 2004. ISSN 0094-8276.

N. M. Shapiro, M. Campillo, L. Stehly, and M. H. Ritzwoller. High-resolution surfacewave tomography from ambient seismic noise. Science, 307(5715):1615-1618, 2005. ISSN 0036-8075.

N. M. Shapiro, M. H. Ritzwoller, and G. D. Bensen. Source location of the 26 sec microseism from cross-correlations of ambient seismic noise. Geophysical Research Letters, 33(18), 2006. ISSN 0094-8276.

P. M. Shearer. Introduction to seismology. Cambridge university press, 2019. ISBN 1107184479

P. M. Shearer, G. A. Prieto, and E. Hauksson. Comprehensive analysis of earthquake source spectra in southern California. Journal of Geophysical Research: Solid Earth, 111(B6), 2006. ISSN 0148-0227.

R. Snieder. Extracting the Green's function from the correlation of coda waves: A derivation based on stationary phase. Physical Review E, 69(4):46610, 2004.

L. Stehly, B. Fry, M. Campillo, N. M. Shapiro, J. Guilbert, L. Boschi, and D. Giardini. Tomography of the Alpine region from observations of seismic ambient noise. Geophysical Journal International, 178(1):338-350, 2009. ISSN 1365-246X.

S. Stein and M. Wysession. An introduction to seismology, earthquakes, and earth structure. John Wiley \& Sons, 2009. ISBN 144431131X.

M. Stirling, M. Gerstenberger, N. Litchfield, G. McVerry, W. Smith, J. Pettinga, and P. Barnes. Seismic hazard of the Canterbury region, New Zealand: New earthquake source model and methodology. Bulletin of the New Zealand Society for Earthquake Engineering, 41(2):51-67, 2008. 
M. W. Stirling, N. J. Litchfield, P. Villamor, R. J. Van Dissen, A. Nicol, J. Pettinga, P. Barnes, R. M. Langridge, T. Little, and D. J. A. Barrell. The Mw 7.82016 Kaikōura earthquake: Surface fault rupture and seismic hazard context. Bulletin of the New Zealand Society for Earthquake Engineering, 50(2):73-84, 2017.

E. Stutzmann, G. Roult, and L. Astiz. GEOSCOPE station noise levels. Bulletin of the Seismological Society of America, 90(3):690-701, 2000. ISSN 1943-3573.

N. Suda, K. Nawa, and Y. Fukao. Earth's background free oscillations. Science, 279 (5359):2089-2091, 1998. ISSN 0036-8075.

T. Taira, P. G. Silver, F. Niu, and R. M. Nadeau. Detecting seismogenic stress evolution and constraining fault zone rheology in the San Andreas Fault following the 2004 Parkfield earthquake. Journal of Geophysical Research: Solid Earth, 113 (B3), 2008. ISSN 0148-0227.

T. Taira, F. Brenguier, and Q. Kong. Ambient noise-based monitoring of seismic velocity changes associated with the $2014 \mathrm{Mw} 6.0$ South Napa earthquake. Geophysical Research Letters, 42(17):6997-7004, 2015.

T. Takano, T. Nishimura, H. Nakahara, Y. Ohta, and S. Tanaka. Seismic velocity changes caused by the Earth tide: Ambient noise correlation analyses of small-array data. Geophysical Research Letters, 41(17):6131-6136, 2014. ISSN 0094-8276.

D. Tocher. Anisotropy in rocks under simple compression. Eos, Transactions American Geophysical Union, 38(1):89-94, 1957. ISSN 0002-8606.

R. Van Dissen and R. S. Yeats. Hope fault, Jordan thrust, and uplift of the seaward Kaikoura Range, New Zealand. Geology, 19(4):393-396, 1991.

J. E. Vidale and Y.-G. Li. Damage to the shallow Landers fault from the nearby Hector Mine earthquake. Nature, 421(6922):524-526, 2003. ISSN 1476-4687.

L. M. Wallace, P. Barnes, J. Beavan, R. Van Dissen, N. Litchfield, J. Mountjoy, R. Langridge, G. Lamarche, and N. Pondard. The kinematics of a transition 
from subduction to strike-slip: An example from the central New Zealand plate boundary. Journal of Geophysical Research: Solid Earth, 117(B2), 2012.

K. Wapenaar. Retrieving the elastodynamic Green's function of an arbitrary inhomogeneous medium by cross correlation. Physical Review Letters, 93(25):254301, 2004.

K. C. Weaver, S. C. Cox, J. Townend, H. Rutter, I. J. Hamling, and C. Holden. Seismological and Hydrogeological Controls on New Zealand-Wide Groundwater Level Changes Induced by the 2016 Mw 7.8 Kaikōura Earthquake. Geofluids, 2019, 2019. ISSN 1468-8115.

R. L. Weaver. Information from seismic noise. Science, 307(5715):1568-1569, 2005. ISSN 0036-8075.

R. L. Weaver and O. I. Lobkis. Ultrasonics without a source: Thermal fluctuation correlations at MHz frequencies. Physical Review Letters, 87(13):134301, 2001.

C. Weemstra, W. Westra, R. Snieder, and L. Boschi. On estimating attenuation from the amplitude of the spectrally whitened ambient seismic field. Geophysical Journal International, 197(3):1770-1788, 2014. ISSN 1365-246X.

U. Wegler and C. Sens-Schönfelder. Fault zone monitoring with passive image interferometry. Geophysical Journal International, 168(3):1029-1033, 2007. ISSN $1365-246 \mathrm{X}$.

U. Wegler, H. Nakahara, C. Sens-Schönfelder, M. Korn, and K. Shiomi. Sudden drop of seismic velocity after the $2004 \mathrm{Mw} 6.6$ mid-Niigata earthquake, Japan, observed with Passive Image Interferometry. Journal of Geophysical Research: Solid Earth, 114(B6), 2009. ISSN 0148-0227.

Y.-M. Wu, T.-l. Teng, T.-C. Shin, and N.-C. Hsiao. Relationship between peak ground acceleration, peak ground velocity, and intensity in Taiwan. Bulletin of the Seismological Society of America, 93(1):386-396, 2003. ISSN 1943-3573.

Z. J. Xu and X. Song. Temporal changes of surface wave velocity associated with 
major Sumatra earthquakes from ambient noise correlation. Proceedings of the National Academy of Sciences, 106(34):14207-14212, 2009. ISSN 0027-8424.

H. Yao, R. D. van Der Hilst, and M. V. De Hoop. Surface-wave array tomography in SE Tibet from ambient seismic noise and two-station analysis-I. Phase velocity maps. Geophysical Journal International, 166(2):732-744, 2006. ISSN 1365-246X.

A. Yates. Seismic velocity changes at White Island volcano, New Zealand, using ten years of ambient noise interferometry (MSc Thesis). Victoria University of Wellington, 2018.

A. S. Yates, M. K. Savage, A. D. Jolly, C. Caudron, and I. J. Hamling. Volcanic, coseismic, and seasonal changes detected at White Island (Whakaari) volcano, New Zealand, using seismic ambient noise. Geophysical Research Letters, 46(1): 99-108, 2019. ISSN 0094-8276.

S. V. Zatsepin and S. Crampin. Modelling the compliance of crustal rock-I. Response of shear-wave splitting to differential stress. Geophysical Journal International, 129(3):477-494, 1997. ISSN 1365-246X.

Z. Zhan, V. C. Tsai, and R. W. Clayton. Spurious velocity changes caused by temporal variations in ambient noise frequency content. Geophysical Journal International, 194(3):1574-1581, 2013. ISSN 1365-246X. 


\section{Appendix A}

\section{Seismic Station Information}

All of the DPRI stations have missing data for at least a few days and for at least one component throughout the testing duration, shown in Figure 3.2. Six of the stations in this Figure were completely unused. These stations and the reasons why they were unused are listed in Table A.1. The main reason why stations were unused was because they contained unreliable data (recording problems), had large amounts of data missing, or they did not contain enough data, either six months before or after the Kaikōura earthquake for the two-year dataset, or two years before and one year after for the seven-year dataset. Out of the nineteen original stations, thirteen stations were used in this thesis for different combinations of station pair cross-components as well as different duration datasets: either the initial four months of testing, the two-year or the seven-year dataset.

\begin{tabular}{|l|l|}
\hline Station & Reason Unused \\
\hline AGR & $\begin{array}{l}\text { Missing data both before and after the Kaikōura earth- } \\
\text { quake }\end{array}$ \\
\hline CRF & Only data from the Kaikōura earthquake until 2018-02 \\
\hline GBR & Only data from the Kaikōura earthquake until 2018-02 \\
\hline GOT & $\begin{array}{l}\text { East and Vertical component data from the Kaikōura } \\
\text { earthquake until 2018-02 }\end{array}$ \\
\hline OGR & Only a few months of data in 2013 \\
\hline TGF & Only data from the Kaikōura earthquake until late 2018 \\
\hline
\end{tabular}

Table A.1: Table of stations with unused data. 


\section{Appendix B}

\section{Final Parameter Choices}

This section contains the parameter choices used within MSNoise to process raw ambient noise data through to velocity details. Parameters for the station pair dataset are found in Table B.1. MSNoise version 1.5 was used for the final processing with version 1.4 being used for the initial testing. The final filter used is shown in Table B.2. 


\begin{tabular}{|c|c|c|}
\hline Parameter & Description & Value \\
\hline analysis_duration & Duration of analysis & $86400 \mathrm{~s}$ \\
\hline autocorr & Compute auto-correlations & $\mathrm{N}$ \\
\hline cc_sampling_rate & Sampling rate for CCF's & $20 \mathrm{~Hz}$ \\
\hline channels & Seismic channels & $\begin{array}{l}\text { EHZ, EHE, } \\
\text { EHN }\end{array}$ \\
\hline components_to_compute & Components to be computed & $\mathrm{ZZ}$ \\
\hline dtt_lag & $\begin{array}{l}\text { How the lag time is defined (dynamic or } \\
\text { static) }\end{array}$ & static \\
\hline dtt_maxdt & Maximum delay time measurement & $0.2 \mathrm{~s}$ \\
\hline dtt_maxerr & Minimum error on delay time measurement & $0.1 \mathrm{~s}$ \\
\hline dtt_mincoh & $\begin{array}{l}\text { Minimum coherence on delay time measure- } \\
\text { ment }\end{array}$ & 0.9 \\
\hline dtt_minlag & Minimum lag for delay time measurement & $15 \mathrm{~s}$ \\
\hline dtt_sides & Which sides of CCFs to use for delay times & both \\
\hline dtt_width & Width of time lag window & $40 \mathrm{~s}$ \\
\hline enddate & End date of data & $2018-02-28$ \\
\hline maxlag & Maximum lag of CCF's & $120 \mathrm{~s}$ \\
\hline mov_stack & Stack size & 30 days \\
\hline preprocess_highpass & Preprocessing high-pass value & $0.01 \mathrm{~Hz}$ \\
\hline preprocess_lowpass & Preprocessing low-pass value & $1.2 \mathrm{~Hz}$ \\
\hline ref_begin & Reference stack start date & 2012-01-01 \\
\hline red_end & Reference stack end data & $2018-02-28$ \\
\hline resampling_method & $\begin{array}{l}\text { Resampling method (resample / decimate / } \\
\text { lanczos) }\end{array}$ & Lanczos \\
\hline stack_method & $\begin{array}{l}\text { Stack Method: Linear or phase weighted } \\
\text { stack }\end{array}$ & Linear \\
\hline startdate & Start date of data & 2012-01-01 \\
\hline windorizing & Windorizing at $\mathrm{N}$ times RMS or -1 is 1-bit. & -1 \\
\hline
\end{tabular}

Table B.1: Station pair pre-processing parameters (MSNoise Version 1.5). These are the parameters used to process the final result for the ZZ component for a 30-day moving window using the full data duration and a seven-year reference period.

\begin{tabular}{|l|l|l|}
\hline Parameter & Description & Value \\
\hline Low & $\begin{array}{l}\text { The lower frequency bound of the whiten } \\
\text { function }\end{array}$ & $0.1 \mathrm{~Hz}$ \\
\hline MWCS low & $\begin{array}{l}\text { The lower frequency bound of the linear re- } \\
\text { gression done in MWCS }\end{array}$ & $0.15 \mathrm{~Hz}$ \\
\hline MWCS high & $\begin{array}{l}\text { The upper frequency bound of the linear re- } \\
\text { gression done in MWCS }\end{array}$ & $0.85 \mathrm{~Hz}$ \\
\hline High & $\begin{array}{l}\text { The upper frequency bound of the whiten } \\
\text { function }\end{array}$ & $0.9 \mathrm{~Hz}$ \\
\hline MWCS wlen & Window length to perform MWCS & $20 \mathrm{~s}$ \\
\hline MWCS step & Step of the windowing procedure in MWCS & $4 \mathrm{~s}$ \\
\hline
\end{tabular}

Table B.2: Station pair dataset filter table (MSNoise Version 1.5). 